\title{
The Moduli Space of Extremal Compact Kähler Manifolds and Generalized Weil-Petersson Metrics
}

\author{
By \\ Akira FuJiki*1 and Georg SCHUMACHER ${ }^{* * 2}$
}

\begin{abstract}
Introduction
A basic problem concerning the classification of compact complex manifolds consists of the construction of a moduli space-a complex space, whose points correspond to isomorphism classes of compact manifolds - say with fixed underlying differentiable manifold $M$. The complex structure of the moduli space reflects the variation of complex structures on $M$ in a holomorphic family. Here, the consideration of a polarization turned out to be essential.

A polarized compact $\mathrm{Kähler} \mathrm{manifold} \mathrm{is} \mathrm{a} \mathrm{pair}(X, \gamma)$ consisting of a compact Kähler manifold $X$ and a Kähler class $\gamma \in H^{2}(X, \mathbb{R})$. In the sequel we fix a compact connected differentiable manifold $M$ and a class $\alpha \in H^{2}(M, \mathbf{R})$, and consider only those polarized manifolds whose underlying differentiable structure is diffeomorphic to $(M, \alpha)$. In [Fu3] and [Sch3] the (coarse) moduli space $\mathfrak{M}=\mathfrak{M}(M, \alpha)$ of polarized, nonuniruled, compact Kähler manifolds was constructed. When $\alpha$ is integral, the moduli space $\mathfrak{M}$ is naturally identified with the moduli space of non-uniruled polarized algebraic manifolds in the sense of algebraic geometry.

The guiding principle of this article is the existence of a natural Kähler structure on the above moduli space, and also when $\alpha$ is integral as above, that of a natural hermitian line bundle whose chern form gives the above Kähler structure. A conceivable approach for this is to represent the given polarization by a distinguished Kähler form such that biholomorphic maps are isometries. In the preceding paper of the second-named author [Sch2] Calabi-Yau metrics were used to construct the moduli space of polarized Kähler manifolds with vanishing first chern class.

In general, a family of such distinguished Kähler metrics ought to yield a strong relationship between infinitesimal variations of complex structures and of metrics tensors in a holomorphic family. Let $\left\{X_{s}\right\}_{s \in S}$ be an effective holomorphic family of compact Kähler manifolds over a reduced complex space $S$ equipped with Kähler metrics $g_{s}$ on each member $X_{s}$. Via the Kodaira-Spencer map we have an inclusion of each tangent
\end{abstract}

Communicated by K. Saito, October 31, 1988.

* Yoshida College, Kyoto University, Kyoto 606, Japan.

** Fakultät für Mathematik, Ruhr-Universität, Bochum, Universitätstr. 150, D-4630 Bochum, Federal Republic of Germany.

1) Partially supported by Alexander von Humboldt-Stiftung.

2) Supported by DFG (Heisenberg-Programm). 
space $T_{s} S$ of $S$ into the cohomology group $H^{1}\left(X_{s}, \Theta_{s}\right)$, where $\Theta_{s}$ is the tangent sheaf of $X_{s}$. Let us now assume that all $g_{s}$ are $\mathbb{K a ̈ h l e r - E i n s t e i n . ~ T h e n ~ t h e ~ c u p ~ p r o d u c t ~ o f ~ a ~}$ harmonic representative of a Kodaira-Spencer class with the metric tensor of the fiber is a symmetric 2-tensor. The latter represents the infinitesimal variation of $g_{s}$ and the $L^{2}$-inner product of such representatives gives rise to a hermitian metric on $S$, which was shown to be $\mathbb{K a ̈ h l e r}$ by $\mathbb{K}$ oiso in [Koi] (at smooth points.) It generalize the classical Weil-Petersson metric for the Teichmüller family of compact $\mathbb{R}$ iemann surfaces, whose $\mathbb{K a ̈ h l e r ~ p r o p e r t y ~ w a s ~ f i r s t ~ p r o v e d ~ b y ~ A h l f o r s ~ [ A ] . ~}$

Now our first idea in this paper is to use more generally the extremal $\mathbb{K a ̈ h l e r}$ metrics in the sense of Calabi [C1][C2] in the above situation-in principle we have then the possibility to treat any polarized compact $\mathbb{K}$ ähler manifold. Extremal $\mathbb{K a ̈ h l e r}$ metrics are exactly the metrics with constant scalar curvature if $X$ is non-uniruled, or more generally, if the automorphism group of $X$ has compact components (manifolds in class $\mathscr{A}$ ). We develop a deformation theory of extremal $\mathbb{K a ̈ h l e r}$ manifolds with fixed polarization. The basic fact we prove is the unique extension property of extremal Kähler metrics in a given polarized family. Using this, we show that the set $\mathfrak{M}_{e}$ of isomorphism classes of extremal compact $\mathbb{K}$ ähler manifolds in $\mathscr{A}$ has a natural structure of a Hausdorff reduced complex space. (The treatment of non-reduced structures could in principle also be done by the method of this paper.)

One of the main results of this paper is the construction of a natural $\mathbb{K a ̈ h l e r}$ metric on this moduli space $\mathfrak{M}_{e}$. For its definition the description of the infinitesimal deformations by harmonic representatives in the sense of Dolbeault-Kodaira does not seem appropriate. Instead, we need to develop a harmonic theory which reflects the relationship between infinitesimal deformations of the complex structure and the metric tensor mentioned above. Harmonic representatives in this sense satisfy a partial differential equation of fourth order rather than of second order. The resulting harmonic space turn out to be the one suggested by the decomposition theorem of Berger-Ebin $[\mathrm{B}-\mathrm{E}]$ (cf. also $[\mathrm{Fu}-\mathrm{Sch}]$ ) in the extremal case. The $L^{2}$-inner product yields a hermitian metric on the base of a universal family $\mathscr{X} \rightarrow S$. This hermitian metric turns out again to be Kähler, and we call it the generalized Weil-Petersson metric. In the KählerEinstein case it coincides with the Weil-Petersson metric mentioned above. Note that $S$ may possibly have singularities. (See Def. 1.1 for the definition of a $\mathbb{K a ̈ h l e r}$ metric on a general complex space.)

As a new aspect, related to our second problem, we consider families of extremal Kähler manifolds $\left\{X_{s}\right\}$ with rational polarization. Then our result is the existence of a hermitain line bundle $(F, k)$ on the base such that its first chern form $c_{1}(F, k)$ coincides with the generalized Weil-Petersson form $\omega_{W P}$ up to a numerical constant. In particular, the $\mathbb{K}$ ähler class of $\omega_{W P}$ is integral up to such a constant. In the Kähler-Einstein case such an $(F, k)$ can even be chosen canonically.

By functoriality, the generalized Weil-Petersson metrics "descends" from the base spaces of the universal families as above to the moduli space $\mathfrak{M}_{e}$. In order to describe this situation, as in the case of the classical Weil-Petersson metric on the moduli space of compact Riemann surfaces, we have to introduce the notion of a $\mathbb{V}$-structure. (In our situation the base of a universal family may have singularities we get a $\mathbb{V}$-space rather than a $V$-manifold.) This implies that the Weil-Petersson form on the moduli space has 
locally a continuous potential which is differentiable with respect to local uniformizing systems of the $V$-structure.

In the case of a rational polarization, combining our method with the classical approach via the Hilbert scheme we show the existence of a hermitian line bundle on the moduli space of rationally polarized extremal Kähler manifolds (in the $V$-sense) whose chern form equals up to a numerical constant the generalized Weil-Petersson form. In particular, all compact complex subspaces of the moduli space are projective.

We shall now explain our method of proof, first in the rationally polarized case. This leads us to a variation of our moduli problem; namely the classification of extremal Hodge manifolds $(X, L, g)$, where $(X, g)$ is an extremal Kähler manifold and $L$ a holomorphic line bundle whose chern class is represented by the Kähler form associated to $g$. One has the notion of a family of extremal Hodge manifolds and a deformation theory. We prove the existence of the moduli space $\mathfrak{M}_{H, e}$ of isomorphism classes of extremal Hodge manifolds in class $\mathscr{A}$. (Here, we distinguish between objects with non-isomorphic line bundles (cf. Def. 6.3).) Also in this case we construct a generalized Weil-Petersson form $\hat{\omega}_{W P}$, and show that up to a constant it is the chern form of a hermitian line bundle $(F, k)$ on the moduli space $\mathfrak{M}_{H, e}$ (in the $V$-sense). In particular, it is a Kähler form.

This follows from two facts: One is the recent result of Bismut-Gillet-Soule [B-G-S] concerning the first chern form of a determinant bundle with Quillen metric-the other is a fiber integral formula, which we show for the generalized Weil-Petersson form on the base space of any family;

$$
\hat{\omega}_{W P}=-1 / n ! \int_{\mathscr{X} / S} 2 \pi c_{1}(\mathscr{X} / S, g) \omega_{\mathscr{X}}^{n}+R /(n+1) ! \int_{\mathscr{X} / S} \omega_{\mathscr{X}}^{n+1} .
$$

Here, $R$ denotes the (constant) scalar curvature (independent of the parameter), and $n$ is the fiber dimension; furthermore, $\omega_{\mathscr{X}}$ is the chern from of some hermitian line bundle on the total space $\mathscr{X}$ which restricts on each fiber to the extremal form. In fact, the right hand side of this formula is interpreted as a chern form of a determinant bundle of the base in the sense of Knudsen-Mumford equipped with the Quillen metric associated with certain virtual hermitian vector bundle on the total space. As a matter of fact, because of the possible singularities we need a generalization of the result of [B-G-S] to the case of singular base space, and in applying the latter the recent result of Varouchas $[V]$ that the above fiber integrals are $\partial \bar{\partial}$-exact on $S$.

The natural map $\mu: \mathfrak{M}_{H, e} \rightarrow \mathfrak{M}_{e}$, which sends the isomorphism class of an extremal Hodge manifold to the class of the underlying extremal Kähler manifold, is proper, holomorphic and open. The fiber dimension $q$ equals $\operatorname{dim} \operatorname{Pic} X_{s}-\operatorname{dim} \operatorname{Aut} X_{s}$. The generalized Weil-Petersson form $\omega_{W P}$ on $\mathfrak{M}_{e}$ equals essentially the fiber integral $\int_{\mu} \hat{\omega}_{W P}^{q+1}$, and this can be interpreted again as the chern form as a certain determinant bundle equipped with the Quillen metric. This in particular shows the desired Kähler property of $\omega_{W P}$ itself.

In the general case where the polarization is not necessarily rational, we follow an analogous (but less intrinsic) method. We only note that if $\omega_{\mathscr{X}}$ is any locally $\partial \bar{\partial}$-exact $(1,1)$-form on $\mathscr{X}$ which restricts to an extremal form on each fiber, we can define the 
associated Weil-Petersson form $\hat{\omega}_{W P}$ and the above fiber integral formula is still true in this case.

In general, such an "admissible" form $\omega_{\mathscr{X}}$ plays an important role in our whole argument, especially via the horizontal distribution it defines on the tangent bundle on $\mathscr{X}$. It turns out that the latter gives rise to harmonic representatives of the $\mathbb{K}$ odairaSpencer classes. In the Kähler-Einstein case we have a canonical choice of such an $\omega_{\mathscr{X}}$ and in this case the associated horizontal lift of the tangent vector coincides with the "canonical lift" of Siu [Si].

As for the relation with the original problem we note that except for the $\mathbb{K}$ ählerEinstein case very little is known about the existence and uniqueness of extremal $\mathbb{K a}$ äler metrics in a given Kähler class. If we denote by $\mathfrak{D M}_{e}^{\prime}$ the open and closed subspace of non-uniruled extremal $\mathbb{K}$ ähler manifolds in $\mathfrak{M}_{e}$, however, the unique extension property of extremal metric implies that the canonical map $\kappa: \mathfrak{D Q}_{e}^{\prime} \rightarrow \mathfrak{M E}$ is an open holomorphic map with discrete fibers.

There is a natural application of the above result to the moduli space of canonically polarized manifolds in algebraic geometry. In particular, we can show that any compact analytic subspace of such a moduli space is always projective (cf. Sect. 11). For a related result see Viehweg [Vi].

In this paper we treat systematically the families with possibly singular base spaces. This is of course indispensable as our final objective is to study the moduli space in general. We would like to emphasize, however, this gives rise to additional difficulties to overcome, e.g., in proving the $\mathbb{K}$ ähler property of generalized WeilPetersson metric. In fact, when the base space is nonsingular, it is also possible to prove the Kähler property by generalizing the method of Koiso [Koi] or $\mathrm{Siu}$ [Si] used in the Kähler-Einstein case.

In [Fu5] extremal Kähler metrics will be studied from the view point of the infinite dimensional moment map.

Now the arrangement of this paper is as follows. In Section 1 after summarizing the basic notions used throughout the paper we introduce the notions of a complex $V$-structure and related objects. In Section 2 we construct an elliptic complex naturally associated to a compact $\mathbb{K a ̈ h l e r}$ manifold with a fixed $\mathbb{K}$ ähler form, which give harmonic representatives of Kodaira-Spencer classes in our deformation theory. In Section 3 we introduce the notions of (metrically) polarized families of Kähler and Hodge manifolds which are to be the basic objects of study in this paper. Then the infinitesimal deformations associated to such families will be studied in Section 4 . In Section 5 we summarize the construction of polarized compact $\mathbb{K a ̈ h l e r}$ and $\mathbb{H o d g e}$ manifolds, and then in Section 6 we construct the local and global moduli spaces for extremal compact Kähler and Hodge manifolds.

In Section 7, by using the results of Sections 2 and 4 we introduce the generalized Weil-Petersson metrics and give statements of our results about the $\mathbb{K}$ ähler property of these metrics. The proofs of these theorems will be given in Sections 8 and 9 . In Section 9 we also give an important relation between two forms $\hat{\omega}_{W P}$ and $\omega_{W P}$ in the Hodge case. In Section 10 by combining the result of Section 7 and the main result of [B-G-S] we construct a natural hermitian line bundle on the base space of any family of Hodge manifolds as mentioned above. In Section 11 we consider the global moduli 
spaces of extremal Hodge and $\mathbb{K a ̈ h l e r ~ m a n i f o l d s ~ a n d ~ o b t a i n ~ t h e ~ g l o b a l ~ v e r s i o n ~ o f ~ t h e ~}$ above result as we have already explained. Finally, Section 12 is devoted to prove a generalization of $[\mathrm{B}-\mathrm{G}-\mathrm{S}]$ to the case of singular base spaces.

\section{Ackmowledgement}

The main part of the present work was done while the first author stayed at University of Münster as a Humboldt research fellow, and at Sonderforschungsbereich 170 at Göttingen. He would like to express his hearty thanks to Professor R. Remmert and to Professor $\mathbb{H}$. Grauert for their hospitality. The second named author was supported by Deutsche Forschungsgemeinschaft (Heisenberg-Programm).

\section{§1。 Prelinminaries}

In this section we first fix our basic terminologies and notations on objects on a general reduced complex space such as $\mathbb{C}^{\infty}$ forms and Kähler metrics. Subsequently, we introduce the notion of a complex $V$-structure generalizing that of a $V$-manifold, which we believe to be most appropriate to describe the structure of the global moduli spaces considered in this paper.

a) We start with the notions of $\mathbb{C}^{\infty}$ functions and forms on a complex space. We denote by $D$ a domain in a complex number space $\mathbb{C}^{m}$ of dimension $m$ (which we do not specify), and by $\mathscr{D}_{D}$ the sheaf of $\mathbb{C}$-valued $\mathbb{C}^{\infty}$ functions on $D$. Let $S$ be a reduced complex space. Then we also denote by $\mathscr{D}_{S}$ the sheaf of $\mathbb{C}$-valued $C^{\infty}$ functions on $S$, which is characterized by the following condition: For any closed analytic embedding $j: U \subseteq D$ of an open subset $U \subseteq S$, the restriction $\mathscr{D}_{S} \mid U$ equals the image of the canonical map $j^{\circ} \mathscr{D}_{D} \rightarrow \mathscr{C}_{S}$, where $j^{\bullet}$ denotes the topological inverse image, and $\mathscr{C}_{S}$ is the sheaf of $\mathbb{C}$-valued continuous functions on $S$. Thus we can identify $\mathscr{D}_{S} \mid U$ with $\left(\mathscr{D}_{D} / \mathscr{J}\right) \mid U$, where $\mathscr{J} \subseteq \mathscr{D}_{D}$ is the subsheaf of functions which vanish identically on $S$. We denote by $\mathscr{D}_{S}(\mathbb{R})$ the subsheaf of $\mathbb{R}$-valued functions.

We next recall the definitions of differential forms. Let $j: U \subset D$ be as above and identify $U$ with $j(U)$. Let $\mathscr{I}$ be the holomorphic ideal sheaf defining $U$ and set $\mathscr{J}=(\mathscr{I}+\overline{\mathscr{I}}) \mathscr{D}_{D}$, where ${ }^{-}$denotes the complex conjugation. For any integer $k>0$ we set $\mathscr{E}_{U}^{k}=\left(\mathscr{E}_{D}^{k} / \mathscr{J}_{\mathscr{E}_{D}^{k}}+d \mathscr{J} \wedge \mathscr{E}_{D}^{k-1}\right) \mid U$, where $\mathscr{E}_{D}^{k}$ is the sheaf of $\mathbb{C}$-valued $C^{\infty} k$-formes on S. For $p, q \geqq 0, p+q=k$, one defines $\mathscr{D}_{U}^{p, q}$ by the natural image of the sheaf $\mathscr{D}_{D}^{p, q}$ of $C^{\infty}(p, q)$-forms on $D$ in $\mathscr{E}_{U}^{k}$. The usual exterior differentiation $d$ on $D$, as well as its $(1,0)$ - and $(0,1)$-parts $\partial$ and $\bar{\partial}$ respectively, give rise to the corresponding exterior differentiations on $\mathscr{E}_{U}^{k}$ and $\mathscr{D}_{U}^{p, q}$ (still denoted by the same letters). These notions actually is independent of the choice of the embedding $j$ as above, so that we obtain global sheaves $\mathscr{E}_{S}^{k}$ and $\mathscr{D}_{S}^{p, q}$ of differential forms on $S$ with global exterior differentiations $d$, and $\partial$ and $\bar{\partial}$ respectively; for instance we have the $\bar{\partial}$-operator $\bar{\partial}: \mathscr{D}_{S}^{p, q} \rightarrow \mathscr{D}_{S}^{p, q+1}$. Sections of $\mathscr{D}_{S}^{p, q}$ are called $C^{\infty}(p, q)$-forms on $S$.

Observe that $(0,0)$-forms give rise to another notion of $C^{\infty}$ functions on $S$. We set $\widehat{\mathscr{D}}_{S}:=\mathscr{E}_{S}^{0}=\mathscr{D}_{S}^{0,0}$; on $U$ we have $\widehat{\mathscr{D}}_{S}=\mathscr{D}_{D} / \mathscr{J}$, and hence there exists a natural epimorphism 


$$
\varepsilon: \widehat{\mathscr{D}}_{\mathbf{S}} \rightarrow \mathscr{D}_{\mathbf{S}}
$$

(If $S$ is locally irreducible, and hence in particular if $S$ is normal, $\varepsilon$ is an isomorphism, but in general not (cf. [M])).

b) Denote by $\mathscr{P}_{S}$ the subsheaf of $\mathscr{D}_{S}(\mathbb{R})$ of pluriharmonic functions on $S$. By definition a pluriharmonic function is locally an imaginary part of a holomorphic function, so that we have the following exact sequence of abelian sheaves

$$
0 \longrightarrow \mathbb{R} \stackrel{j}{\longrightarrow} \mathcal{O}_{S} \stackrel{\operatorname{Im}}{\longrightarrow} \mathscr{P}_{S} \longrightarrow 0,
$$

where $j$ is the natural inclusion.

Proposition $\mathbb{1}_{0} \mathbb{H}_{0}$ Let $S_{\text {reg }}$ be the set of smooth points of $S$. Let $\varphi \in \Gamma\left(S, \mathscr{D}_{S}(\mathbb{R})\right)$ be a $C^{\infty}$ function whose restriction to $S_{\mathrm{reg}}$ is pluriharmonic. Then $\varphi$ is pluriharmonic on the whole S.

Proof. We extend the argument in the proof of [Fu2; Lemma 6]. Since the problem is local, we fix a point $o \in S$ and prove that $\varphi$ is pluriharmonic in a neighborhood of $o$. Let $r: \tilde{S} \rightarrow S$ be a resolution of $S$ and set $E=r^{-1}(o)$. Then we consider the following commutative diagram with exact rows

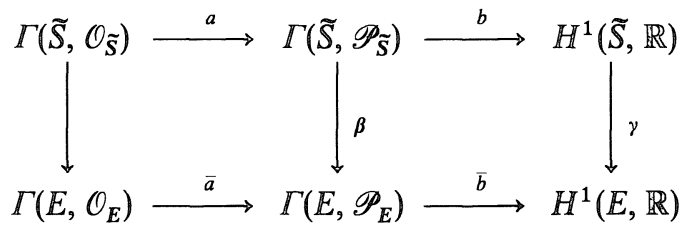

arising from (1.1), where the vertical arrows are restriction maps.

Suppose first that $a$ is surjective. Then, since $\tilde{\varphi}:=r^{*} \varphi$ is in $\Gamma\left(\widetilde{S}, \mathscr{P}_{\tilde{S}}\right)$, we can find a holomorphic function $f$ on $\widetilde{S}$ with $\operatorname{Im} f=\varphi$, determined up to additive real constants on each connected component of $\tilde{S}$. By adjusting these constants we may assume that $f$ descends to a (continuous) meromorphic function $f$ on $S$ whose imaginary part coincides with $\varphi$. Since $\varphi$ is of class $C^{\infty}$, by a theorem of Spallek [Sp; Satz 4.2] $f$ is holomorphic, and hence $\varphi$ is pluriharmonic, on the whole $S$.

It remains to prove the surjectivity of $a$. First note that from the compactness of $E$ the surjectivity of $\bar{a}$ follows at once. Hence, $\bar{b}$, and therefore $\bar{b} \beta=\gamma b$ also, is the zero map. On the other hand, if we replace $S$ by a suitable neighborhood of $o$, we may assume that $\gamma$ is isomorphic. Thus $b$ is the zero map and $a$ is surjective.

Corollary 1.2. 1) Two locally $\partial \bar{\partial}$-exact real $C^{\infty}(1,1)$-forms which coincide on $S_{\text {reg }}$ coincides on the whole S. 2) The map $\partial \bar{\partial}: \widehat{\mathscr{D}}_{S} \rightarrow \mathscr{D}_{S}^{1,1}$ descends to $\partial \bar{\partial}: \mathscr{D}_{S} \rightarrow \mathscr{D}_{S}^{1,1}$ with respect to $\varepsilon: \widehat{\mathscr{D}}_{\mathbf{S}} \rightarrow \mathscr{D}_{\mathbf{S}}$.

Proof. 1) The problem is local. Let $\psi_{1}$ and $\psi_{2}$ be $\mathbb{R}$-valued $C^{\infty}$ functions on $S$ with $\partial \bar{\partial} \psi_{1}=\partial \bar{\partial} \psi_{2}$ on $S_{\text {reg. }}$. Then by applying Proposition 0.1 to $\psi:=\psi_{1}-\psi_{2}$ we get that the equality is even true on the whole $S$. 2) Since the support of the kernel of $\varepsilon$ is contained in $S-S_{\text {reg }}, \partial \bar{\partial}$ vanishes identically on $\mathrm{Ker} \varepsilon$ by the above proposition.

We denote by 


$$
\Phi_{S}:=i \partial \bar{\partial} \mathscr{D}_{\mathbf{S}}(\mathbb{R}) \subseteq \mathscr{D}_{\mathbf{S}}^{1,1}(\mathbb{R})
$$

the subsheaf of locally $\partial \bar{\partial}$-exact real $(1,1)$-forms on $S$. The above proposition yields the following exact sequence

$$
0 \longrightarrow \mathscr{P}_{S} \longrightarrow \mathscr{D}_{S}(\mathbb{R}) \stackrel{i \partial \bar{\partial}}{\longrightarrow} \Phi_{S} \longrightarrow 0
$$

as in the smooth case.

We further observe the exact sequence

$$
0 \longrightarrow S^{1} \longrightarrow \mathcal{O}_{S}^{*} \stackrel{\log ||}{\longrightarrow} \mathscr{P}_{S} \longrightarrow 0,
$$

and the induced cohomology sequence

$$
\longrightarrow H^{1}\left(S, \mathcal{O}_{S}^{*}\right) \stackrel{\hat{c}}{\longrightarrow} H^{1}\left(S, \mathscr{P}_{S}\right) \longrightarrow H^{2}\left(S, S^{1}\right) \longrightarrow
$$

where $S^{1}=\{z \in \mathbb{C} ;|z|=1\}$, identified with the constant sheaf on $S$. For any holomorphic line bundle $L$ on $S$

$$
\hat{c}(L) \in H^{1}\left(S, \mathscr{P}_{S}\right)
$$

is called the refined chern class of $L$. If, further, $h$ is a hermitian metric on $L$, we set

$$
c_{1}(L, h)=i / 2 \pi \operatorname{curv} h=i / 2 \pi \bar{\partial} \partial \log h
$$

and call it the first chern form of the hermitian line bundle $(L, h)$, where curv denotes the curvature form. Then $c_{1}(L, h)$ is a section of $\Phi_{S}$ and we have

$$
\hat{c}(L)=\delta_{S}\left(c_{1}(L, h)\right),
$$

where $\delta_{S}: \Gamma\left(S, \Phi_{S}\right) \rightarrow H^{1}\left(S, \mathscr{P}_{S}\right)$ is the coboundary map arising from (1.2).

c) Let $f: \mathscr{X} \rightarrow S$ be a proper smooth morphism of connected and reduced complex spaces with connected fibers. For any $C^{\infty}(p, q)$-form $\alpha$ on $\mathscr{X}$ we can define its fiber integral $\beta=\int_{\mathscr{X} / S} \alpha$ along the fibers of $f$, which is a $C^{\infty}(p-n, q-n)$-form on $S$, where $n$ is the dimension of the fibers. For instance, in the case $(p, q)=(n+1, n+1)$ we obtain a $(1,1)$-form and we are interested in the condition for $\beta$ to be locally $\partial \bar{\partial}$-exact on $S$. Concerning this question the following special case of a recent result of Varouchas [V] turns out to be very useful for our purpose.

Proposition 1.3. Let $f: \mathscr{X} \rightarrow S$ and $n$ be as above. Let $\omega_{0}, \ldots, \omega_{n}$ be real $C^{\infty}$ $(1,1)$-forms on $\mathscr{X}$ which are locally $\partial \bar{\partial}$-exact on $\mathscr{X}$. Then the fiber integral $\int_{\mathscr{X} / S} \omega_{0} \wedge \cdots \wedge \omega_{n}$ is again a locally $\partial \bar{\partial}$-exact real $(1,1)$-form on $S$, i.e., a section of $\Phi_{S}$.

Proof. The problem is local. So by restricting $S$ we may assume that $H^{i}(\mathscr{X}, \mathbb{R})=$ 0 for $i \geqq 2 n+1$ and $H^{j}(\mathscr{X}, \mathscr{F})=0$ for $j \geqq n+1$ and for any coherent analytic sheaf $\mathscr{F}$ on $\mathscr{X}$. Then by [V; Th. 2] there exists a real $C^{\infty}(n, n)$-form $\chi$ on $\mathscr{X}$ such that

$$
\omega_{0} \wedge \cdots \wedge \omega_{n}=i \partial \bar{\partial} \chi
$$

on the whole $\mathscr{X}$. In fact, in [V] this is proved for the case where $\omega_{0}=\cdots=\omega_{n}$ and $\omega=\omega_{i}$ is a Kähler form; however, the proof clearly shows that it suffices only to assume that $\omega$ is locally $\partial \bar{\partial}$-exact and real. Thus for any real numbers $r_{0}, \ldots, r_{n},\left(r_{1} \omega_{1}+\cdots+r_{n} \omega_{n}\right)^{n+1}$ 
is $\partial \bar{\partial}$-exact on the whole $\mathscr{X}$. From this one deduces that the same is true for $\omega_{0}^{i_{0}} \wedge \cdots \wedge \omega_{n}^{i_{n}}$ for any $0 \leqq i_{0}, \ldots, i_{n}$ with $i_{0}+\cdots+i_{n}=n+1$. Hence, (1.7) holds. Finally, we get

$$
\int_{\mathscr{X} / S} \omega_{0} \wedge \cdots \wedge \omega_{n}=i \int_{\mathscr{X} / S} \partial \bar{\partial} \chi=i \partial \bar{\partial} \int_{\mathscr{X} / S} \chi .
$$

The proposition follows.

d) Let $f: \mathscr{X} \rightarrow S$ be a smooth morphism of reduced complex spaces. We can then define the relative holomorphic tangent (resp. cotangent) bundle $T_{\mathscr{X} / S}$ (resp. $T_{\mathscr{X} / S}^{*}$ ) as a holomorphic vector bundle on $\mathscr{X}$. Then $\bigwedge^{p} \mathbb{T}_{\mathscr{X} / S}^{*} \otimes \bigwedge^{q} \bar{T}_{\mathscr{X} / S}^{*}$ is a complex $C^{\infty}$ vector bundle on $\mathscr{X}$. We denote by $\mathscr{D}_{\mathscr{X} / S}^{p, q}$ the sheaf of $\mathbb{C}^{\infty}$ sections of this bundle. Sections of $\mathscr{D}_{\mathscr{X} / S}^{p, q}$ are called $C^{\infty}$ relative $(p, q)$-forms on $\mathscr{X}$ over $S$. We set $\mathscr{D}_{\mathscr{X} / S}^{q}=\mathscr{D}_{\mathscr{X} / S}^{0, q}$ and $\mathscr{E}_{\mathscr{X} / S}^{k}:=$ $\underset{p+q=k}{\oplus} \mathscr{D}_{\mathscr{X} / S}^{p, q}$. Then we set $\mathscr{D}_{\mathscr{X} / S}^{0,0}=\mathscr{E}_{\mathscr{X} / S}^{0}=\mathscr{D}_{\mathscr{X}}$. Also we denote by $\Omega_{\mathscr{X} / S}^{p}$ the sheaf of germs of holomorphic sections of $\bigwedge^{p} \mathbb{T}_{\mathscr{X} / S}^{*}$ (i.e., the sheaf of relative holomorphic $p$-forms), and by $\mathcal{O}_{\mathscr{X} / S}$ the subsheaf of $\mathscr{D}_{\mathscr{X}}$ consisting of those functions which are holomorphic when restricted to each fiber.

Since $f$ is smooth, we have differential operators

$$
d_{\mathscr{X} / S}: \mathscr{E}_{\mathscr{X} / S}^{k} \rightarrow \mathscr{E}_{\mathscr{X} / S}^{k+1}, \partial_{\mathscr{X} / \mathbf{S}}: \Omega_{\mathscr{X} / S}^{p} \rightarrow \Omega_{\mathscr{X} / S}^{p+1}, \bar{\partial}_{\mathscr{X} / \mathbf{S}}: \mathscr{D}_{\mathscr{X} / S}^{q} \rightarrow \mathscr{D}_{\mathscr{X} / S}^{q+1}
$$

as in the absolute case, which give rise to complexes on $\mathscr{X}$

$$
\begin{aligned}
& d_{\mathscr{X} / S}: 0 \rightarrow f^{\circ} \mathscr{D}_{S} \rightarrow \mathscr{D}_{\mathscr{X}} \stackrel{d}{\rightarrow} \mathscr{E}_{\mathscr{X} / S}^{1} \stackrel{d}{\rightarrow} \mathscr{E}_{\mathscr{X} / S}^{2} \rightarrow \\
& \partial_{\mathscr{X} / \mathbf{S}}: 0 \rightarrow f^{\circ} \mathcal{O}_{S} \rightarrow \mathcal{O}_{\mathscr{X}} \stackrel{\stackrel{\partial}{\rightarrow}}{\rightarrow} \Omega_{\mathscr{X} / S}^{1} \stackrel{\vec{\partial}}{\rightarrow} \Omega_{\mathscr{X} / S}^{2} \rightarrow \\
& \bar{\partial}_{\mathscr{X} / \mathbf{S}}: 0 \rightarrow \mathcal{O}_{\mathscr{X} / S} \rightarrow \mathscr{D}_{\mathscr{X}} \stackrel{\bar{\partial}}{\rightarrow} \mathscr{D}_{\mathscr{X} / S}^{1} \stackrel{\bar{\partial}}{\rightarrow} \mathscr{D}_{\mathscr{X} / S}^{2} \rightarrow
\end{aligned}
$$

where $f^{\circ}$ denotes the topological inverse image, and in each line the second arrow is the natural inclusion.

Proposition $\mathbb{1}_{\text {.4. }}$ The above three sequences are all exact, i.e., the relative versions of Poincaré and Dolbeault lemma hold.

In the Poincare cases this follows from the observation that the construction of the homotopy for $d$ or $\partial$ in the absolute case depends smoothly on the parameter. The Dolbeault case is due to Andreotti and Grauert $[\mathbb{A}-\mathbb{G} ; \S 7, b), c)]$ when $\mathbb{S}$ is nonsingular. The proof in the general case, which can also take care of the Poincaré cases, will be given in the Appendix.

The functions which are locally imaginary parts of functions in $\mathcal{O}_{\mathscr{X} / \mathrm{S}}$ form a subsheaf $\mathscr{P}_{\mathscr{X} / S}$ of $\mathscr{D}_{S}(\mathbb{R})$. Sections of $\mathscr{P}_{\mathscr{X} / S}$ are called relative pluriharmonic functions on $\mathscr{X}$ over $S$. We have the obvious exact sequence

$$
0 \longrightarrow f^{\circ} \mathscr{D}_{S}(\mathbb{R}) \stackrel{j_{0}}{\longrightarrow} \mathcal{O}_{\mathscr{X} / \mathbf{S}} \stackrel{\operatorname{Im}}{\longrightarrow} \mathscr{P}_{\mathscr{X} / \mathbf{S}} \longrightarrow 0
$$

where $j_{0}$ is the natural inclusion. We also set

$$
\Phi_{\mathscr{X} / S}=\operatorname{Ker}\left(d_{\mathscr{X} / S}: \mathscr{D}_{\mathscr{X} / S}^{1,1} \rightarrow \mathscr{E}_{\mathscr{X} / S}^{3}\right) \text {. }
$$


Then Proposition 1.4 yields as in the absolute case:

Lemma 1.5. The following sequence is exact:

$$
0 \longrightarrow \mathscr{P}_{\mathscr{X} / S} \stackrel{j_{1}}{\longrightarrow} \mathscr{D}_{\mathscr{X}}(\mathbb{R}) \stackrel{i \partial_{\mathscr{X} / S} \bar{\partial}_{X / S}}{\longrightarrow} \Phi_{\mathscr{X} / S} \longrightarrow 0
$$

where $j_{1}$ is the natural inclusion.

e) We next define the notion of a Kähler metric on a reduced complex space $S$. We start with that of a hermitian metric. Denote by $T=T_{S} \rightarrow S$ the tangent linear fiber space, whose fibers over $s \in S$ are naturally identified with the Zariski tangent space $T_{s} S$ of $S$ at $s$ (cf. [Fi]). With respect to an embedding j: $U \subset D$ of an open subset $U \subseteq S$ into a domain $D$ in some $\mathbb{C}^{m}$ we have a canonical embedding of the restriction $T \mid U$ into the holomorphic tangent bundle $T_{D}$ of $D$ :

$$
\begin{array}{ccc}
T \mid U \subset & T_{D} \\
\downarrow & & \downarrow \\
U & & \\
& \hookrightarrow & D .
\end{array}
$$

Then a hermitian metric on $S$ is by definition a collection $h=\left\{h_{s}\right\}_{s \in S}$ of hermitian inner products $h_{s}$ on $T_{s} S$ It is said to be of class $C^{\infty}$ if for any point $s$ of $S$, if we take $U$ sufficiently small around $s$ in (1.11) $\left\{h_{s}\right\}_{s \in U}$ can be extended to a $C^{\infty}$ hermitian metric $h_{D}$ on $D$.

Definition 1.1. A $C^{\infty}$ hermitian metric $h$ on $S$ is called a $C^{\infty}$ Kähler metric if for any point $s \in S$ there exist an embedding of a neighborhood $U$ of $s$ into a domain $D \subseteq \mathbb{C}^{m}$ and a $\mathbb{C}^{\infty}$ Kähler metric $h_{D}$ which extends $h \mid U$ (as a hermitian metric).

We have also an equivalent notion of Kähler forms. First of all, note that any real $C^{\infty}(1,1)$-form $\omega$ on $S$ determines at each point $s \in S$ a hermitian form (not necessary positive definite) $h(\omega)_{s}$ on $T_{s} S$.

Definition 1.2. A Kähler form on $S$ is a locally $\partial \bar{\partial}$-exact, real $C^{\infty}(1,1)$-form $\omega$ on $S$ such that the induced hermitian form $h(\omega)_{s}$ is positive definite at each point $s$ of $S$.

Remark 1.1. 1) By the definition any Kähler form $\omega$ is locally written in the form $\omega=i \partial \bar{\partial} \psi$ for some $C^{\infty}$ strictly plurisubharmonic function $\psi$, which is determined up to additions of pluriharmonic functions. Thus the notion of a Kähler form given above coincides with the standard one used, e.g., in [Fu2] or [V], which originates from Moishezon.

2) A Kähler form induces naturally a Kähler metric as is clear from the definitions. More precisely, by using 1) of Corollary 1.2 one sees easily that this sets up a natural bijective correspondence between Kähler forms and Kähler metrics as in the smooth case.

Let $f: \mathscr{X} \rightarrow S$ be a smooth morphism of reduced complex spaces. Then a horizontal distribution for $f$ is a collection $T^{H} \mathscr{X}=\left\{T_{x}^{H}\right\}_{x \in \mathscr{X}}$ of subspaces $T_{x}^{H}$ of the Zariski tangent spaces $T_{x} \mathscr{X}$ of $\mathscr{X}$ such that $f$ induces an isomorphism $T_{x}^{H} \rightarrow T_{f(x)} S$. $T^{H} \mathscr{X}$ is 
called of class $C^{\infty}$ if for any point $x \in \mathscr{X}$ we can find a neighborhood $\mathbb{V}$ and a realization of $f$ by a projection

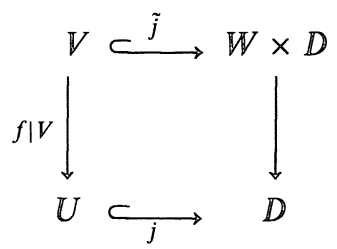

such that $T^{H} \mathscr{X}$ is induced by a $C^{\infty}$ horizontal distribution for the projection $\pi$ in the obvious sense, where $U \subseteq S$ and $j$ are as in (1.11), $W$ is a manifold and $\tilde{j}$ is a closed embedding.

Let now $\omega_{\mathscr{X}}$ be a $C^{\infty}(1,1)$-form on $\mathscr{X}$ which induces a Kähler form on each fiber of $f$. Then define for any $x$ the subspace $T_{x}^{H}\left(\omega_{\mathscr{X}}\right)$ of $T_{x} \mathscr{X}$ by the $\omega_{\mathscr{X}}$-orthogonal complement of $T_{x} \mathscr{X}_{s}$, where $s=f(x)$. Then:

$$
T^{H}\left(\omega_{\mathscr{X}}\right):=\bigcup_{x \in \mathscr{C}} T_{x}^{H}\left(\omega_{\mathscr{X}}\right)
$$

is a $C^{\infty}$ horizontal distribution for $f$.

Definition 1.3. Suppose that $\omega_{\mathscr{X}}$ is a Kähler form with the associated $\mathbb{K}$ ähler metric $h_{\mathscr{X}}$ and $S$ is given a hermitian metric $h_{S}$. Then $f$ is said to be a Kähler submersion if the linear isomorphism $T_{x}^{H}\left(\omega_{\mathscr{X}}\right) \widetilde{\rightarrow} T_{f(x)} S$ is a hermitian isometry for any $x$.

In this case we know that $h_{S}$ also is a Kähler metric at least on the smooth locus $S_{\text {reg. }}$ (See $[$ Wa $]$.)

f) For the description of the natural Kähler structure on the global moduli spaces considered in this paper it seems most natural to introduce the notion of a "complex $V$-structure" generalizing the notion of a $V$-manifold in the sense of Satake (cf. $[\mathrm{Ba}])$.

Definition 1.4. Let $Y$ be a reduced complex space. Then:

1) A local (analytic) Galois cover of $Y$ is a pair $\mathscr{F}=(\pi: \tilde{U} \rightarrow U, G)$ consisting of a holomorphic map $\pi: \widetilde{U} \rightarrow U$ of a connected complex space $\tilde{U}$ onto an open subset $U$ of $Y$ and of a finite group $G$ acting biholomorphically on $\tilde{U}$ over $U$ such that $\pi$ induces an

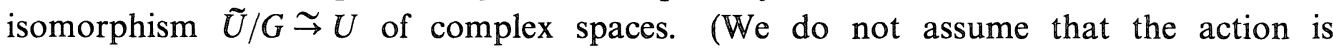
effective.)

2) Let $\mathscr{F}^{\prime}=\left(\pi^{\prime}: \tilde{U}^{\prime} \rightarrow U^{\prime}, G^{\prime}\right)$ be another local Galois cover of $Y$ with $U^{\prime} \leqq U$, then a morphism of $\mathscr{F}^{\prime}$ into $\mathscr{F}$ is a commutative diagram of complex spaces

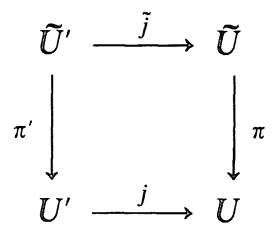

where $j$ is the inclusion and $\tilde{j}$ is an open embedding, together with an injective homomorphism $\mu: G^{\prime} \rightarrow G$ such that $\tilde{j}$ is $\mu$-equivariant. Any such $\tilde{j}$ is said to be 
associated to $j$. If such a morphism exists, $\mathscr{F}$ and $\mathscr{F}^{\prime}$ are said to be compatible.

3) A complex $V$-structure on $Y$ is a collection

$$
\mathscr{V}=\left\{\mathscr{F}_{\alpha}=\left(\pi_{\alpha}: \tilde{U}_{\alpha} \rightarrow U_{\alpha}, G_{\alpha}\right)\right\}_{\alpha \in A}
$$

of local Galois covers of $Y$ such that 1) $\left\{U_{\alpha}\right\}_{\alpha \in A}$ forms a basis for open sets of $Y$, and 2) $\mathscr{F}_{\alpha}$ and $\mathscr{F}_{\beta}$ are compatible whenever $U_{\alpha} \subseteq U_{\beta}$.

4) Let $Y^{\prime}$ be another complex space with a complex $V$-structure $\mathscr{V}^{\prime}=\left\{\mathscr{F}_{\gamma}^{\prime}=\left(\pi^{\prime}\right.\right.$ : $\left.\left.\widetilde{U}_{\gamma}^{\prime} \rightarrow U_{\gamma}^{\prime}, G_{\gamma}^{\prime}\right)\right\}$. Then a morphism $f: Y \rightarrow Y^{\prime}$ of complex spaces is said to be $V$-smooth if for any point $y \in Y$ there exist $\mathscr{F}_{\alpha} \in \mathscr{V}, \mathscr{F}_{\gamma}^{\prime} \in \mathscr{V}^{\prime}$ and a homomorphism $\mu_{\alpha \gamma}: G_{\alpha} \rightarrow G_{\gamma}^{\prime}$ such that $f(y) \in U_{\gamma}^{\prime}, y \in U_{\alpha}$, and $f \mid U_{\alpha}$ lifts to a $\mu_{\alpha \gamma}$-equivariant smooth morphism $\tilde{U}_{\alpha} \rightarrow \widetilde{U}_{\gamma}^{\prime}$.

Let $Y$ be a complex space with a complex $V$-structure $\mathscr{V}=\left\{\mathscr{F}_{\alpha}=\left(\pi_{\alpha}: \widetilde{U}_{\alpha} \rightarrow\right.\right.$ $\left.\left.U_{\alpha}, G_{\alpha}\right)\right\}$.

Definition 1.5. 1) A Kähler V-metric on $Y$ is a collection $h=\left\{h_{\alpha}\right\}$ of $G_{\alpha}$-invariant Kähler metrics $h_{\alpha}$ on $\tilde{U}_{\alpha}$ such that if $U_{\alpha} \subseteq U_{\beta}$ then $\tilde{j}_{\alpha \beta}^{*} h_{\beta}=h_{\alpha}$, where $\tilde{j}_{\alpha \beta}: \tilde{U}_{\alpha} \rightarrow \tilde{U}_{\beta}$ is associated to the inclusion $U_{\alpha} \subseteq U_{\beta}$. In a similar manner we can define differential $V$-forms and Kähler $V$-forms etc. on $Y$.

2) Let $Y$ and $Y^{\prime}$ be complex spaces with complex $V$-structure and with Kähler $V$-metrics. A morphism $f: Y \rightarrow Y^{\prime}$ is said to be a Kähler $V$-submersion if it is $V$-smooth and a lift $\widetilde{U}_{\alpha} \rightarrow \widetilde{U}_{\gamma}^{\prime}$ as in 4) of Definition 1.4 can be taken to be a Kähler submersion.

Let $F$ be a holomorphic line bundle on $Y$. Then to each $F_{\alpha}:=\pi_{\alpha}^{*}\left(F \mid U_{\alpha}\right)$ the action of $G_{\alpha}$ on $\tilde{U}_{\alpha}$ lifts naturally. Moreover, any embedding $\tilde{j}_{\alpha \beta}: \widetilde{U}_{\alpha} \rightarrow \widetilde{U}_{\beta}$ associated to an inclusion $U_{\alpha} \subseteq U_{\beta}$ induces naturally an open embedding $\hat{j}_{\alpha \beta}: F_{\alpha} \subseteq F_{\beta}$ of bundle spaces.

Definition 1.6. A $C^{\infty}$ hermitian $V$-metric on $F$ is a collection $h=\left\{h_{\alpha}\right\}$ of $G_{\alpha}$ invariant $C^{\infty}$ hermitian metrics $h_{\alpha}$ on $F_{\alpha}$ such that $\hat{j}_{\alpha \beta}^{*} h_{\beta}=h_{\alpha}$ for any $\hat{j}_{\alpha \beta}$ as above. In this case the collection $\left\{c_{1}\left(F_{\alpha}, h_{\alpha}\right)\right\}$ of the first chern forms of $\left(F_{\alpha}, h_{\alpha}\right)$ determines a $(1,1)$-V-form on $Y$, which we shall denote by $c_{1}(F, h)$ and call it the first chern $V$-form of $(F, h)$.

The following notion will also be useful in studying the moduli spaces.

Definition 1.7. Let $Y$ be as above. An abelian $V$-sheaf on $Y$ is a collection $L=\left\{L_{\alpha}\right\}$ of sheaves of abelian groups $L_{\alpha}$ on $\tilde{U}_{\alpha}$ with a lift of the $G_{\alpha}$-action to $L_{\alpha}$ and with the following property: For any open embedding $\tilde{j}_{\alpha \beta}: \widetilde{U}_{\alpha} \hookrightarrow \tilde{U}_{\beta}$ associated to an inclusion $U_{\alpha} \subseteq U_{\beta}$, we are given an isomorphism $v_{\alpha \beta}: L_{\alpha} \widetilde{\sim} \tilde{j}_{\alpha \beta}^{*} L_{\beta}$ which is $\mu_{\alpha \beta^{-}}$ equivariant, where $\mu_{\alpha \beta}$ : $G_{\alpha} \rightarrow G_{\beta}$ is the given homomorphism, such that for any inclusions $U_{\alpha} \subseteq U_{\beta} \subseteq U_{\gamma}$ and for any open embeddings $\tilde{j}_{\alpha \beta}, \tilde{j}_{\beta \gamma}$ and $\tilde{j}_{\alpha \gamma}$ associated to these inclusions with $\tilde{j}_{\beta \gamma} \tilde{j}_{\alpha \beta}=\tilde{j}_{\alpha \gamma}$, we get $\tilde{j}_{\alpha \beta}^{*}\left(v_{\beta \gamma}\right) v_{\alpha \beta}=v_{\alpha \gamma}$.

From the definition we have:

Lemma 1.6. Let $\mathscr{L}=\left\{\mathscr{L}_{\alpha}\right\}$ be an abelian $V$-sheaf on $Y$ as above. Let $\overline{\mathscr{L}}_{\alpha}:=$ $\pi_{\alpha *}^{G_{\alpha}} \mathscr{L}_{\alpha}$ be the $G_{\alpha}$-invariant direct image sheaves on $U_{\alpha}$. Then $\overline{\mathscr{L}}_{\alpha}$ for all $\alpha$ patch together naturally to a global abelian sheaf $\overline{\mathscr{L}}$ on $Y$. 
Examples of abelian $V$-sheaves arise from sheaves naturally attached to each $\widetilde{U}_{\alpha}$, such as $\mathscr{O}_{\tilde{U}_{\alpha}}, \mathscr{D}_{\tilde{U}_{\alpha}, q}^{p, q} \mathscr{D}_{\tilde{U}_{\alpha}}, \Phi_{\tilde{U}_{\alpha}}, \mathscr{P}_{\tilde{U}_{\alpha}}$ etc. We denote by $\Phi_{Y}^{V}$ and $\mathscr{D}_{Y}^{V}$ respectively the abelian sheaves on $Y$ determined by $\Phi_{\tilde{U}_{\alpha}}$ and $\mathscr{D}_{\tilde{U}_{\alpha}}$ and Lemma 1.6. Note that for $\mathcal{O}_{\tilde{U}_{\alpha}}$ and $\mathscr{P}_{\tilde{U}_{\alpha}}$ the resulting sheaves are just $\mathcal{O}_{Y}$ and $\mathscr{P}_{Y}$ respectively. The exact sequence (1.2) induces an exact sequence

$$
0 \rightarrow \mathscr{P}_{Y} \rightarrow \mathscr{D}_{Y}^{V}(\mathbb{R}) \rightarrow \Phi_{Y}^{V} \rightarrow 0
$$

on $Y$.

Note that by definition we may consider a $\mathbb{K}$ ähler $V$-form $\omega_{Y}$ as a section of $\Phi_{Y}^{V}$.

Definnition $\mathbb{1}_{\text {. }}$. The image $\hat{c}\left(\omega_{Y}\right) \in \mathbb{H}^{1}\left(Y, \mathscr{P}_{Y}\right)$ by the coboundary map $\Gamma\left(\mathbb{Y}, \Phi_{Y}^{V}\right) \rightarrow$ $H^{1}\left(Y, \mathscr{P}_{Y}\right)$ induced by $(1.14)$ is called the refined $\mathbb{K}$ ähler class of $\omega_{Y}$. Its image $\omega_{Y} \in$ $H^{2}(Y, \mathbb{R})$ by the coboundary map arising from (1.1) is called the Kähler class of $\omega_{Y}$. The same terminology is used also for an (ordinary) complex space. (In this case (1.14) is reduced to (1.2).)

If $\mathscr{L}_{\alpha}$ are all invertible $\mathcal{O}_{\tilde{U}_{\alpha}}$-modules in Definition 1.7 , and $\mathbb{L}_{\alpha}$ is the corresponding line bundle, we call the collection $\left\{L_{\alpha}\right\}$ a line $V$-bundle on $Y$. The hermitian $V$-metric on a line $V$-bundle is also defined analogously to Definition 1.6. If $m$ is a common multiple of the orders of $G_{\alpha}$ for all $\alpha,\left\{L_{\alpha}^{m}\right\}$ descends to an ordinary line bundle on $Y$; indeed, in this case $\pi_{\alpha^{\alpha^{\alpha}}}^{G_{\mathcal{L}_{\alpha}}}$ are again invertible $\mathcal{O}_{U_{\alpha}}$-modules.

Definition 1 . 9 . Let $Z$ be an (ordinary) complex space. Then a morphism $f: \mathbb{Z} \rightarrow$ $Y$ of complex spaces is said to be a $V$-morphism if for any point $z \in \mathbb{Z}$, there exist a

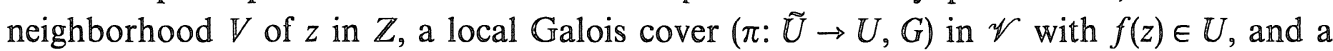
morphism $\tau: V \rightarrow \widetilde{U}$ such that $f \mid V=\pi \tau$.

In this case we can define the pull-backs $f^{*} B$ of $V$-objects $B=\left\{B_{\alpha}\right\}$ on $\mathbb{Y}$ such as $C^{\infty} V$-forms, line bundles, hermitian metric etc.

Proposiđịn 1.7. Let $Y$ be a reduced complex space with a complex V-structure. Suppose that there exists a $\mathbb{K}$ ähler $\mathbb{V}$-form $\omega_{Y}$ on $\mathbb{Y}$ whose $\mathbb{K}$ ähler class $\gamma$ is integral, i.e., $\gamma$ is in the image of $\mathbb{H}^{2}(X, \mathbb{Z}) \rightarrow \mathbb{H}^{2}(X, \mathbb{R})$. Then there exists a positive line bundle $\mathbb{L}$ with $c_{1}(L)=\gamma$. In particular, if $Y$ is compact, $Y$ is projective.

Proof. Let $\mathscr{V}=\left\{\mathscr{F}_{\alpha}=\left(\pi_{\alpha}: \widetilde{U}_{\alpha} \rightarrow U_{\alpha}, G_{\alpha}\right)\right\}$ be the complex $V$-structure on $\mathbb{Y}$. Suppose that $\omega_{Y}$ is given by a system $\left\{\omega_{\alpha}\right\}$ of $G_{\alpha}$-invariant $\mathbb{K} a ̈ h l e r$ forms $\omega_{\alpha}$ on $\widetilde{U}_{\alpha}$. For $\alpha$ with small $U_{\alpha}$ we may write $\omega_{\alpha}=i \partial \bar{\partial} \tilde{\psi}_{\alpha}$ for some $G_{\alpha}$-invariant $C^{\infty}$ strongly plurisubharmonic (psh) function $\tilde{\psi}_{\alpha}$ on $\tilde{U}_{\alpha}$. Then $\tilde{\psi}_{\alpha}$ descends to a continuous strongly psh function $\psi_{\alpha}$ on $U_{\alpha}$. If $U_{\alpha} \subseteq U_{\beta}$, then $\psi_{\alpha}-\psi_{\beta}$ is $\mathbb{C}^{\infty}$ pluriharmonic on $U_{\alpha}$. In fact, take an open embedding $\tilde{j}_{\alpha \beta}: \widetilde{U}_{\alpha} \rightarrow \widetilde{U}_{\beta}$ associated to the inclusion $\mathbb{U}_{\alpha} \subseteq U_{\beta}$. Then $\tilde{\psi}_{\alpha}-\tilde{j}_{\alpha \beta}^{*} \tilde{\psi}_{\beta}$ can be written as a real part of a $G_{\alpha}$-invariant holomorphic function $\tilde{h}_{\alpha \beta}$ on $\tilde{U}_{\alpha}$; the latter descends to a holomorphic function $h_{\alpha \beta}$ on $\mathbb{U}_{\alpha}$ and we see that $\psi_{\alpha}-\psi_{\beta}$ is the real part of $h_{\alpha \beta}$ on $U_{\alpha}$. Thus $\psi_{\alpha}-\psi_{\beta}$ is $C^{\infty}$ pluriharmonic on $U_{\alpha}$. Then by Theorem 1 of [V] there exists a $C^{\infty}$ strongly psh function $\psi_{\alpha}^{\prime}$ on $\mathbb{U}_{\alpha}$ such that $\psi_{\alpha}^{\prime}-\psi_{\beta}^{\prime}=h_{\alpha \beta}$. Thus we get a global $\mathbb{C}^{\infty} \mathbb{K} a ̈ h l e r$ form $\omega_{Y}^{\prime}$ by setting $\omega_{Y}^{\prime} \mid U_{\alpha}=i \partial \bar{\partial} \psi_{\alpha}^{\prime}$ such that $\gamma=\left[\omega_{Y}^{\prime}\right]$ as both are the image of $\left\{h_{\alpha \beta}\right\} \in H^{1}\left(\left\{U_{\alpha}\right\}, \mathscr{P}_{Y}\right)$. 
Now we observe the following commutative diagram of cohomology exact sequences on $Y$

$$
\begin{aligned}
& H^{1}\left(\mathcal{O}_{Y}\right) \rightarrow H^{1}\left(\mathcal{O}_{Y}^{*}\right) \rightarrow H^{2}(\mathbb{Z}) \\
& \downarrow \downarrow \\
& H^{1}\left(\mathscr{P}_{Y}\right)=H^{1}\left(\mathscr{P}_{Y}\right) \\
& \downarrow \downarrow \\
& H^{2}(\mathbb{Z}) \rightarrow H^{2}(\mathbb{R}) \rightarrow H^{2}\left(S^{1}\right) .
\end{aligned}
$$

The assumption that $\gamma$ is integral shows that the element $h \in H^{1}\left(\mathscr{P}_{Y}\right)$ determined by $\left\{h_{\alpha \beta}\right\}$ is in the image of $H^{1}\left(\mathcal{O}_{Y}^{*}\right)$. Let $L$ be a line bundle with the refined chern class $h$. Then it is easy to construct hermitian metric $k$ on $L$ such that $c_{1}(L, k)=\omega_{Y}^{\prime}$ (cf. Prop. 3.10 below). Thus $L$ is positive, and further, is ample if $Y$ is compact. (See [Gr].)

\section{§2. Elliptic Complex Associated to a Compact Kähler Manifold}

Fix a connected compact Kähler manifold $X$ and a Kähler form $\omega$ on it throughout this section. Accordingly, for any abelian sheaf $\mathscr{F}$ we write $H^{q}(\mathscr{F})$ for $H^{q}(X, \mathscr{F})$ for short, and similarly, for a $C^{\infty}$ vector bundle $E$ write $\Gamma(E)$ for the space of $C^{\infty}$ sections. Let $T$ and $T^{*}$ be holomorphic tangent and cotangent bundles of $X$ respectively. We denote by $A^{q}=A^{q}(X)$ (resp. $\left.D^{q}=D^{q}(X)\right)$ the space $A^{q}=\Gamma\left(T \otimes \wedge^{q} \bar{T}^{*}\right.$ ) (resp. $D^{q}=$ $\Gamma\left(\bigwedge^{q} \bar{T}^{*}\right)$ ) of vector valued $C^{\infty}(0, q)$-forms (resp. ordinary $(0, q)$-forms) on $X$ which form as usual the Dolbeault complex with respect to $\bar{\partial}$, where the bar denotes the complex conjugate.

Now in this section, we shall construct a certain elliptic complex $B^{*}=B^{*}(X, \omega)$ naturally associated to the Kähler manifold $(X, \omega)$ (depending also on the Kähler form $\omega$ ), essentially as a subcomplex of $A^{*}$. The cohomology group $H^{q}\left(B^{\circ}\right)$ of this complex will be interpreted as a certain sheaf cohomology group $H^{q}\left(\Sigma_{\gamma}\right)$, where $\Sigma_{\gamma}$ is defined by the Atiyah sequence associated to the Kähler class $\gamma$ of $\omega$ (cf. (2.13)). Since $H^{q}\left(\Sigma_{\gamma}\right)$ is considered as the space of certain infinitesimal deformations associated to $(X, \omega)$ (cf. Sect. 4) this means that we get natural harmonic representatives for such infinitesimal deformations. If $\omega$ is of constant scalar curvature, these representatives are exactly the ones coming from the decomposition theorem of Berger-Ebin [B-E] formulated in terms of Riemannian geometry.

Denote by $g$ the Kähler metric associated to $\omega$. Then $g$ and $\omega$ give natural identifications of tangent and cotangent bundles; we denote these isomorphisms $T \rightarrow$ $\bar{T}^{*}$ and $\bar{T}^{*} \rightarrow T$ by $\downarrow_{g}, \downarrow_{\omega}$ and $\uparrow_{g}, \uparrow_{\omega}$ respectively.

We first define a bundle map

$$
l_{\omega}^{q}: T \otimes \bigwedge^{q} \bar{T}^{*} \rightarrow \bigwedge^{q+1} \bar{T}^{*}, \quad q \geqq 0,
$$

by the composition

$$
T \otimes \bigwedge^{q} \bar{T}^{*} \stackrel{\downarrow_{\omega} \otimes i d}{\longrightarrow} \bar{T}^{*} \otimes \bigwedge^{q} \bar{T}^{*} \subset^{i d \times j_{q}} \longrightarrow \bar{T}^{* \otimes(q+1)} \stackrel{A}{\longrightarrow} \bigwedge^{q+1} \bar{T}^{*},
$$


where $j_{q}: \bigwedge^{q} \bar{T}^{*} \rightarrow \bar{T}^{* \otimes q}$ is the natural inclusion and $A$ is the alternation operator. We denote by the same letter $l_{\omega}^{q}$ the induced linear map

$$
l_{\omega}^{q}: A^{q} \rightarrow D^{q+1} .
$$

From the definition the following is immediate.

Lemma 2.1. $l_{\omega}^{q}$ are all surjective in (2.1), and hence also in (2.3).

Lemma 2.2. The linear maps $\imath_{\omega}^{q}, q \geqq 0$, induces a homomorphism $i_{\omega}^{\circ}: A^{\circ} \rightarrow \mathbb{D}^{\circ+1}$ of Dolbeault complexes.

Proof. Write $\imath=l_{\omega}^{q}$. We have to show that $\bar{\partial} \imath(V)=\imath \bar{\partial}(V)$ for all $V \in A^{q}$. Since the problem is local, we may assume that $V$ is of the form $a \otimes b$, where $a$ is a local nonvanishing holomorphic vector field and $b$ is a $C^{\infty}(0, q)$-form. Note first that $l(a \otimes b)=\imath(a) \otimes b$ by the definition and that $\bar{\partial}(a \otimes b)=a \otimes \bar{\partial} b$. We have thus only to show that $\bar{\partial}(l(a) \otimes b)=l(a) \wedge \bar{\partial} b$. This clearly follows from $\bar{\partial} l(a)=0$. So we shall show the latter. It suffices to show that for any local vector fields $U, V$ of type $(1,0)$ such that $a, U, V$ all commute, we have $(d l(a))(U, V)=0$. In fact, noting that $l(a)(W)=\omega(a, W)$ for any $W$ by the definition, we have

$$
\begin{aligned}
2(d(l(a))(U, V) & =U(l(a)(V))-V(l(a)(U)) \\
& =U \omega(a, V)-V \omega(a, U)+a \omega(U, V) \\
& =3(d \omega)(U, a, V)=0 .
\end{aligned}
$$

Now for $q>0$ we define $B^{q}=B^{q}(X, \omega)$ by the kernel of $\imath_{\omega}^{q}$ and set $\bar{\partial}_{B}=\bar{\partial}: B^{q} \rightarrow$ $B^{q+1}$. Namely

$$
B^{q}=\operatorname{Ker}\left(l_{\omega}^{q}: A^{q} \rightarrow D^{q+1}\right)
$$

We further set

$$
B^{0}=B^{0}(X, \omega)=C^{\infty}(X)
$$

where $C^{\infty}(X)$ is the space of $\mathbb{C}$-valued $C^{\infty}$ functions. We then get the following commutative diagram of exact sequences

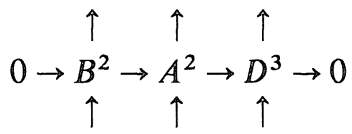

$$
\begin{aligned}
& 0 \rightarrow B^{1} \rightarrow A_{\uparrow}^{1} \rightarrow \underset{\uparrow}{D^{2}} \rightarrow 0 \\
& \underset{\uparrow}{A^{0} \stackrel{\uparrow}{\rightarrow} D^{1}} \\
& 0 \rightarrow D^{0} \\
& \uparrow \\
& 0 \text {. }
\end{aligned}
$$


Then we see that the composite map

$$
L: D^{0} \stackrel{\bar{\partial}}{\longrightarrow} D^{1} \stackrel{\uparrow_{\omega}}{\longrightarrow} A^{0} \stackrel{\bar{\partial}}{\longrightarrow} A^{1}
$$

has image in $B^{1}$. Hence we set $\bar{\partial}_{B}:=L: B^{0} \rightarrow B^{1}$;

$$
\bar{\partial}_{B}=\bar{\partial} \uparrow_{\omega} \bar{\partial}=i \bar{\partial} \uparrow_{g} \bar{\partial} .
$$

By Lemma 2.2 and the definition of $\bar{\partial}_{B},\left(B^{\circ}, \bar{\partial}_{B}\right)$ is a complex. We note the cohomology exact sequence which arises from (2.4):

$$
\longrightarrow H^{q}\left(\mathcal{O}_{X}\right) \stackrel{\varepsilon^{q}}{\longrightarrow} H^{q}\left(B^{\circ}\right) \stackrel{a^{q}}{\longrightarrow} H^{q}\left(\Theta_{X}\right) \stackrel{w^{q}}{\longrightarrow} H^{q+1}\left(\mathcal{O}_{X}\right) \longrightarrow
$$

where we have used the canonical isomorphisms $H^{q}\left(A^{*}\right) \cong H^{q}\left(\Theta_{X}\right)$ and $H^{q}\left(D^{\bullet}\right) \cong H^{q}\left(\mathcal{O}_{X}\right)$.

Proposition 2.3. $\left(B^{\circ}, \bar{\partial}_{B}\right)$ is an elliptic complex.

Proof. Let $x$ be any point of $X$. Fix any nonzero real cotangent vector $v$ at $x$. Then we have the following commutative diagram of symbol sequence of complexes $A^{\circ}, B^{\circ}$, and $D^{\circ}$ at $(x, v)$ :

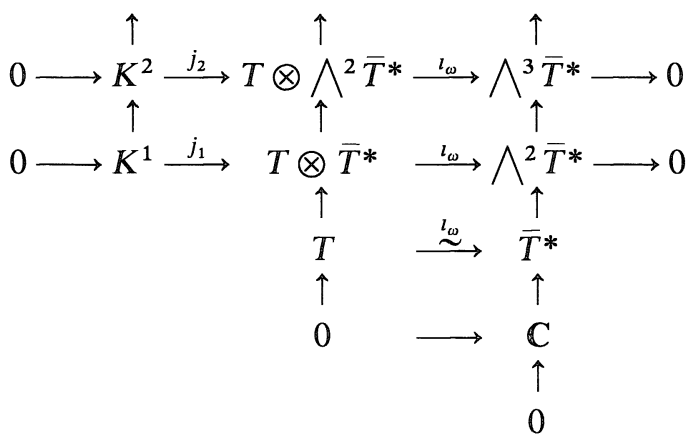

where $K^{q}, q \geqq 1$, is the kernel of $l_{\omega}^{q}$ in (2.1) and $j^{q}$ are the natural inclusions. Since the Dolbeault complexes are elliptic, the middle and the right vertical sequences are exact. It follows that the left vertical sequence is also exact except at degree 0 and 1 . The exactness at degree at 0 or 1 follows immediately if we note that $\sigma_{v}\left(\bar{\partial}_{B}\right)=i \sigma_{v}\left(\bar{\partial}_{A}\right) \uparrow_{\omega} \sigma_{v}\left(\bar{\partial}_{D}\right)$, where $\sigma_{v}$ denotes the symbol.

We now consider elements of $A^{1}:=\Gamma\left(T \otimes \bar{T}^{*}\right)$ as a homomorphism $\bar{T} \rightarrow T$ of vector bundles. Then $B^{1}$ is by definition the subspace of elements $\varphi$ of $A^{1}$ which is skew symmetric with respect to $\omega$;

$$
B^{1}=B^{1}(X, \omega)=\left\{\varphi \in A^{1} ; \omega(\varphi(U), V)+\omega(U, \varphi(V))=0\right\}
$$

or

$$
=\left\{\varphi \in A^{1} ; g(\varphi(U), V)=g(U, \varphi(V))\right\},
$$

where $U, V \in A^{0}=\Gamma(T)$, and note that $g(U, V)=\omega(U, J V)$ with $J$ the complex structure of $X$.

By virtue of the above proposition we may apply the usual harmonic theory to the complex $\left(B^{\circ}, \bar{\partial}_{B}^{\circ}\right)$. First of all, since $X$ is given a Kähler metric, $B^{q}$, as well as $A^{q}$ and $D^{q}$, 
carries a natural hermitian $L^{2}$-inner product. For instance if $\varphi$ and $\psi$ are elements of $B^{1} \subseteq A^{1}$ considered as homomorphisms $\bar{T} \rightarrow T$ as above, this inner product is given explicitly by

$$
(\varphi, \psi)=\int_{X} \operatorname{tr}(\varphi \bar{\psi}) \omega^{[n]},
$$

where $\omega^{[n]}:=\omega^{n} / n$ ! is the volume form associated to the Kähler metric $g$, and the trace tr is taken in End $T$. Let $\bar{\partial}_{B}^{*}$ be the formal adjoint of $\bar{\partial}_{B}$ with respect to $g$. In our case, the Laplacian $\square^{q}=\square_{B}^{q}$ is defined in each degree as follows:

$$
\begin{aligned}
& \square^{0}=\bar{\partial}_{B}^{*} \bar{\partial}_{B}, \quad \square^{1}=\bar{\partial}_{B} \bar{\partial}_{B}^{*}+\left(\bar{\partial}^{*} \bar{\partial}\right)^{2} \\
& \square^{q}=\left(\overline{\partial \bar{\partial}^{*}}\right)^{2}+\left(\bar{\partial}^{*} \bar{\partial}\right)^{2}, \quad q \geqq 2 .
\end{aligned}
$$

(We shall write $\bar{\partial}=\bar{\partial}_{B}$ for degree $\geqq 1$.) We denote by $\mathscr{H}_{B}^{q}$ the space of harmonic forms. For instance we have

$$
\begin{aligned}
& \mathscr{H}_{B}^{0}=\left\{f \in C^{\infty}(X) ; \bar{\partial}_{B} f=0\right\}, \\
& \mathscr{H}_{B}^{1}=\left\{\varphi \in B^{1} ; \square^{1} \varphi=0\right\}=\left\{\bar{\partial} \varphi=\bar{\partial}_{B}^{*} \varphi=0\right\} .
\end{aligned}
$$

An immediate consequence of the harmonic theory is as follows (cf. e.g. [We]).

Theorem 2.4. $\mathscr{H}_{B}^{q}$ are all finite dimensional and the natural map $v^{q}: \mathscr{H}_{B}^{q} \rightarrow H^{q}\left(B^{\circ}\right)$ are isomorphisms.

Note that with respect to local coordinates, say $z_{1}, \ldots, z_{n}$ on $X$, if $\varphi \in B^{1}$ is written as $\varphi=\sum \varphi_{\beta}^{\alpha} \partial / \partial z_{\alpha} \otimes d \bar{z}_{\beta}, \bar{\partial}_{B}^{*}$ takes the form

$$
\sum \varphi_{\bar{\delta} \bar{\beta}}^{\bar{\beta} \bar{\delta}}=0, \quad \varphi_{\bar{\delta} \bar{\beta}}=\sum g_{\alpha \bar{\delta}} \varphi_{\bar{\beta}}^{\alpha},
$$

where $g_{\alpha \bar{\beta}}$ is the metric tensor and $\varphi_{\alpha \bar{\beta}}=\varphi_{\beta \bar{\alpha}}$ by (2.7).

We further define subspaces $\hat{\mathscr{H}}_{B}$ of $A^{0}$ and $\overline{\mathscr{H}}_{B}^{1}$ of $\mathscr{H}_{B}^{1}$ by

$$
\hat{\mathscr{H}}_{B}:=\left\{\xi \in A^{0} ; \bar{\partial} \xi \in \mathscr{H}_{B}^{1}\right\},
$$

and

$$
\overline{\mathscr{H}}_{B}^{1}:=\bar{\partial} \hat{\mathscr{H}}_{B} \subseteq \mathscr{H}_{B}^{1}
$$

Definition 2.1. Define $\check{\mathscr{H}}_{B}^{1}$ to be the orthogonal complement of $\overline{\mathscr{H}}_{B}^{1}$ in $\mathscr{H}_{B}^{1} ; \breve{\mathscr{H}}_{B}^{1}=$ ${ }^{\perp} \overline{\mathscr{H}}_{B}^{1}$. We call $\check{\mathscr{H}}_{B}^{1}$ the essential harmonic space in $B^{1}$.

We shall give a cohomological interpretation of $\breve{\mathscr{H}}_{B}^{1}$. We denote by $\gamma$ the Kähler class of $\omega ; \gamma=[\omega]$. We first note that $w=w^{1}: H^{1}\left(\Theta_{X}\right) \rightarrow H^{2}\left(\mathcal{O}_{X}\right)$ is given by the cup product with $\gamma$ considered as an element of $H^{1}\left(\Omega_{X}^{1}\right)$, as follows easily from the definition of the sequence (2.5). Therefore, we get the following exact sequence

$$
H^{1}\left(\mathcal{O}_{X}\right) \stackrel{\varepsilon}{\longrightarrow} H^{1}\left(B^{\circ}\right) \stackrel{a}{\longrightarrow} H^{1}\left(\Theta_{X}\right)_{\gamma} \longrightarrow 0
$$

where $\varepsilon=\varepsilon^{1}, a=a^{1}$ and

$$
H^{1}\left(\Theta_{X}\right)_{\gamma}:=\operatorname{Ker}\left(\gamma: H^{1}\left(\Theta_{X}\right) \rightarrow H^{2}\left(\mathcal{O}_{X}\right)\right)
$$


Note that if we set ' $\hat{\mathscr{H}}_{B}=\hat{\mathscr{H}}_{B} / \uparrow_{\omega} \bar{\partial} \mathscr{H}_{B}^{0}, \bar{\partial}$ induces a linear map ' $\hat{\mathscr{H}}_{B} \rightarrow \mathscr{H}_{B}^{1}$, which will still be denoted by $\bar{\partial}$.

Theorem 2.5. 1) There exists a linear isomorphism $\delta: H^{1}\left(\mathcal{O}_{X}\right) \stackrel{\sim}{\rightarrow} \hat{\mathscr{H}}_{B}$ such that $v \bar{\partial} \delta=\varepsilon$, where $v=v^{1}$ as above. 2) The map $a^{\prime}:=a v: \mathscr{H}_{B}^{1} \rightarrow H^{1}\left(\Theta_{X}\right)_{\gamma}$ induces by restriction to $\breve{\mathscr{H}}_{B}^{1}$ a natural isomorphism $\check{\mu}$ : $\check{\mathscr{H}}_{B}^{1} \rightarrow H^{1}\left(\Theta_{X}\right)_{\gamma}$ such that $a^{\prime}=\check{\mu} p$, where $p: \breve{H}_{B}^{1} \rightarrow \check{\mathscr{H}}_{B}^{1}$ is the orthogonal projection.

Proof. For simplicity, we write $\uparrow=\uparrow_{\omega}$. Let $G$ and $H$ be the Green operator and the harmonic projection operator respectively associated to the harmonic theory on $B^{\circ}$. Then we define $\hat{\delta}: Z^{1}(X) \rightarrow \hat{\mathscr{H}}_{B}$ by

$$
\hat{\delta}(\varphi)=\uparrow\left(\varphi-\bar{\partial} \bar{\partial}_{B}^{*} G \bar{\partial} \uparrow \varphi\right),
$$

where $Z^{1}(X)$ is the space of $\bar{\partial}$-closed $(0,1)$-forms. In fact, if we set $\xi=\bar{\partial} \uparrow \varphi$ we get

$$
\bar{\partial} \hat{\delta}(\varphi)=\xi-\bar{\partial}_{B} \bar{\partial}_{B}^{*} G \bar{\partial} \xi=H \xi ;
$$

hence the image of $\hat{\delta}$ is certainly in $\hat{\mathscr{H}}_{B}$. Moreover, if we set $\varphi=\bar{\partial} f, f \in C^{\infty}(X)$, in the definition of $\hat{\delta}$ we get

$$
\uparrow\left(\bar{\partial} f-\bar{\partial} \bar{\partial}_{B}^{*} G \bar{\partial}_{B} F\right)=\uparrow(\bar{\partial} f-\bar{\partial}(f-H f))=\uparrow \bar{\partial} H f \in \uparrow \bar{\partial} \mathscr{H}_{B}^{0} .
$$

Thus, $\hat{\delta}$ induces a linear map $\delta: H^{1}\left(\mathcal{O}_{X}\right) \rightarrow^{\prime} \hat{\mathscr{H}}_{B}$. The formula (2.11) shows then the commutativity $v \bar{\partial} \delta=\varepsilon$ in view of the definition of $\varepsilon$. If $\delta(\varphi)=0$, then $\varphi=\bar{\partial} \bar{\partial}_{B}^{*} G \bar{\partial} \uparrow \varphi+$ $\bar{\partial} f$ for some $f \in \mathscr{H}_{B}^{0}$; hence $\delta$ is injective. On the other hand, for any $\xi \in \hat{\mathscr{H}}_{\boldsymbol{B}}$, if we set $\xi=\uparrow \varphi$ for a unique $\varphi \in Z^{1}(X)$, then since $G \bar{\partial} \uparrow \varphi=G \bar{\partial} \xi=0$, we have $\xi=\delta(\varphi)$. Hence $\delta$ is surjective. This proves 1 ).

2) then follows from 1) together with (2.10) and the fact that the orthogonal complement $\overline{\mathscr{H}}_{B}^{1}$ of $\check{\mathscr{H}}_{B}^{1}$ is precisely the kernel of $a^{\prime}$ by 1 ).

Proposition 2.6. In the above proposition suppose further that $(X, \omega)$ is of constant scalar curvature. Then $\uparrow_{\omega}$ induces an isomorphism $\mathscr{H}^{1} \widetilde{\sim}^{\prime} \hat{\mathscr{H}}_{B}$ such that $\uparrow_{\omega}=\delta j$, where $\mathscr{H}^{1}$ is the space of harmonic $(0,1)$-forms in $D^{1}$, and $j: \mathscr{H}^{1} \rightarrow H^{1}\left(\mathcal{O}_{X}\right)$ is the natural isomorphism.

Proof. It suffices to show that $\hat{\delta} \mid \mathscr{H}^{1}=\uparrow$ in the above proof. By the definition of $\hat{\delta}$ this follows if we show that $\bar{\partial}_{B}^{*} \bar{\partial} \uparrow \varphi=0$ for $\varphi \in \mathscr{H}^{1}$. Let $\square^{\prime \prime}$ be the Laplacian operating on $(0,1)$-forms and $\rho$ the Ricci form of $(X, \omega)$. By contraction with $\uparrow \varphi, \rho$ defines again a $(0,1)$-form, say $Q(\varphi)$. Then we have (cf. [K; p. 158])

$$
0=\square^{\prime \prime} \varphi=\sum \varphi_{\bar{\beta} ; \alpha}^{\alpha}+Q(\varphi), \quad \varphi=\sum_{\beta} \varphi_{\beta} d \bar{z}_{\beta},
$$

where in our notations we may further write

$$
\sum \varphi_{\bar{\beta} ; \alpha}^{\alpha} d z^{\bar{\beta}}=\downarrow_{g} \bar{\partial}^{*} \bar{\partial} \uparrow_{g} \varphi=\downarrow \bar{\partial}^{*} \bar{\partial} \uparrow \varphi,
$$

where $\downarrow=\downarrow_{\omega}$. On the other hand, since $(X, \omega)$ is of constant scalar curvature $\bar{\partial}^{*} Q(\varphi)=0$ (cf. [K; p. 98]). Hence, $\bar{\partial}_{B}^{*} \bar{\partial} \uparrow \varphi=\left(\bar{\partial}^{*} \downarrow \bar{\partial}^{*}\right) \bar{\partial} \uparrow \varphi=0$.

In the Kähler-Einstein case there is a comparison result of our essential harmonic space $\check{\mathscr{H}}_{B}^{1}$ and the harmonic space $\mathscr{H}_{B}^{1}$ with certain spaces of harmonic forms in 
$A^{1}$. So suppose that $(X, \omega)$ is $\mathbb{K a ̈ h l e r - E i n s t e i n . ~ R e c a l l ~ t h a t ~ w e ~ h a v e ~ t h e ~ f o l l o w i n g ~ t w o ~}$ cases:

a) $\gamma=r c_{1}(X)$ for some real number $r \neq 0$, and

b) $\gamma$ is arbitrary and $c_{1}(X)=0$.

Let $\mathscr{H}_{A}^{1}$ be the space of harmonic 1 -forms in $A^{1}$ with respect to $g$. Let ${ }^{\prime} \mathscr{H}_{A}^{1}:=\mathscr{H}_{A}^{1} \cap B^{1}$ and " $\mathscr{H}_{A}^{1}$ be the subspaces of $\mathscr{H}_{A}^{1}$ of those elements which are symmetric and antisymmetric with respect to $g$ respectively (cf. (2.7)). Then by [Koi] (cf. also [Sch4] [Si]) we have the following:

Proposition 2.7. In the case a) we have $\mathscr{H}_{A}^{1}={ }^{\prime} \mathscr{H}_{A}^{1}$. In the case b) we have the direct sum decomposition $\mathscr{H}_{A}^{1}={ }^{\prime} \mathscr{H}_{A}^{1} \oplus " \mathscr{H}_{A}^{1}$, and " $\mathscr{H}_{A}^{1}$ is mapped isomorphically to $H^{2}\left(\mathcal{O}_{X}\right)$ via the natural linear map $\mathscr{H}_{A}^{1} \underset{\sim}{\rightarrow} H^{1}\left(\Theta_{X}\right) \stackrel{w}{\longrightarrow} H^{2}\left(\mathcal{O}_{X}\right)$.

Proof. See [Koi; Prop. 8.2, 8.3]. As for the last assertion he proves that " $\mathscr{H}_{A}^{1}$ is in general isomorphic to the space of parallel $(0,2)$-forms, considered as a subspace of $H^{2}\left(\mathcal{O}_{X}\right)$. But if $c_{1}(X)=0$, every anti-holomorphic 2-form is parallel (cf. [Bea]). From this, the last assertion follows.

Proposiư⿴囗十⺝ 2.8. Suppose that $(X, \omega)$ is Kähler-Einstein as above. Then the natural inclusion $B^{1} \hookrightarrow A^{1}$ induces a hermitian isomorphism of hermitian spaces $h: \breve{\mathscr{H}}_{B}^{1} \rightarrow \mathscr{H}_{A}^{1}$ which fit into the following commutative diagram with isomorphic arrows

$$
\begin{gathered}
\check{\mathscr{H}}_{B}^{1} \underset{\rightarrow}{\longrightarrow} H^{1}\left(\Theta_{X}\right)_{\gamma} \\
\imath \downarrow \quad \imath \downarrow \\
\mathscr{H}_{A}^{1} \stackrel{\sim}{\rightarrow} \mathbb{H}^{1}\left(\Theta_{X}\right)
\end{gathered}
$$

in the case a). Similarly, in the case b) we get a hermitian isomorphism $h^{\prime}: \check{\mathscr{H}}_{B}^{1} \stackrel{\sim}{\rightarrow}^{\prime} \mathscr{H}_{A}^{1}$ of hermitian spaces.

Proof. By the definition of the harmonic spaces we have clearly the inclusion ${ }^{\prime} \mathscr{H}_{A}^{1} \subseteq \mathscr{H}_{B}^{1}$. Further, by the fact that $\mathscr{H}_{A}^{1}$ is orthogonal to the image of $A^{0}$ by $\bar{\partial}$ we get ${ }^{\prime} \mathscr{H}_{A}^{1} \subseteq \check{\mathscr{H}}_{B}^{1}$. On the other hand, we have in general

$$
\operatorname{dim} \check{\mathscr{H}}_{B}^{1}=\operatorname{dim} H^{1}\left(\Theta_{X}\right)_{\gamma} \leqq \operatorname{dim} H^{1}\left(\Theta_{X}\right)=\operatorname{dim} \mathscr{H}_{A}^{1} .
$$

Hence, by Proposition 2.7 we have $\breve{\mathscr{H}}_{B}^{1}=\mathscr{H}_{A}^{1}$ in the case a) and $\check{\mathscr{H}}_{B}^{1}={ }^{\prime} \mathscr{H}_{A}^{1}$ in the case b). The commutativity of (2.12) is also obvious.

We now go back to a general $(X, \omega)$ with the Kähler class $\gamma=[\omega]$ as before. Consider now $\gamma$ as an element of $H^{1}\left(\Omega_{X}^{1}\right)=H^{1}\left(\mathscr{H}_{o m_{\mathcal{O}_{X}}}\left(\Theta_{X}^{1}, \mathcal{O}_{X}\right)\right)$. Then it defines a locally free extension

$$
0 \rightarrow \mathcal{O}_{X} \rightarrow \Sigma_{\gamma} \rightarrow \Theta_{X} \rightarrow 0
$$

of $\Theta_{X}$ by $\mathcal{O}_{X}$. We then get the associated cohomology exact sequence

$$
\longrightarrow H^{1}\left(\mathcal{O}_{X}\right) \stackrel{v}{\longrightarrow} H^{1}\left(\Sigma_{\gamma}\right) \stackrel{u}{\longrightarrow} H^{1}\left(\Theta_{X}\right) \longrightarrow H^{2}\left(\mathcal{O}_{X}\right) \longrightarrow,
$$

where the coboundary maps $H^{q}\left(\Theta_{X}\right) \rightarrow H^{q+1}\left(\mathcal{O}_{X}\right)$ are obtained by a cup product with $\gamma ; \cup \gamma$. 
If $\gamma=c_{1}(L)$ is the chern class of a holomorphic line bundle $L$ on $X$, then (2.13) is nothing but the Atiyah sequence associated to the principal $\mathbb{C}^{*}$-bundle $L^{\times}:=L-0 \rightarrow$ $X$. (See [At].) In this case $\Sigma_{L}:=\Sigma_{\gamma}$ is identified with the sheaf (on $X$ ) of $\mathbb{C}^{*}$-invariant holomorphic vector fields on $L$; in other words, $\Sigma_{L}$ is just the $\mathbb{C}^{*}$-invariant direct image sheaf

$$
\Sigma_{L}=\pi_{*}^{\mathbb{C}^{*}} \Theta_{L}
$$

We put $Q:=T L^{\times} / \mathbb{C}^{*}$, the quotient of the holomorphic tangent bundle $T L^{\times}$of $L^{\times}$ by the natural $\mathbb{C}^{*}$ action. Then $Q$ is a vector bundle on $X$ with $\mathcal{O}_{X}(Q)=\Sigma_{L}$. In general we denote also by $Q$ the vector bundle on $X$ corresponding to $\Sigma_{\gamma}$. Then the sequence (2.13) is associated to an exact sequence of vector bundles

$$
0 \longrightarrow 1_{X} \stackrel{\imath}{\longrightarrow} Q \stackrel{\pi}{\longrightarrow} T \longrightarrow 0 .
$$

Then in accordance with the case of line bundles we call any $C^{\infty}$ splitting of the sequence $(2.16)$ a connection of type $(1,0)$ (of the class $\gamma$ ). Such a connection is thus given by a homomorphism $\theta: Q \rightarrow 1_{X}$ with $\theta l=i d$. As usual $\theta$ is represented by a system $\alpha=\left\{\alpha_{i}\right\}$ of $C^{\infty}(1,0)$-forms $\alpha_{i}$ with respect to a suitable open covering $\mathscr{U}=\left\{U_{i}\right\}$ of $X$ such that $\psi_{i}-\psi_{j}=c_{i j}$, where $c=\left\{c_{i j}\right\}$ is a representing cocycle of $\gamma$ in $H^{1}\left(\Omega_{X}^{1}\right)$.

For the given Kähler form $\omega$ such connections with "curvature form" $2 \pi / i \omega$ are constructed as follows. Let $\mathscr{U}=\left\{U_{i}\right\}$ be a sufficiently fine Stein covering of $X$ so that we may write $\omega=2 \pi / i \partial \bar{\partial} \psi_{i}$ for some $\mathbb{R}$-valued $C^{\infty}$ functions $\psi_{i}$ on $U_{i}$ and $\psi_{i}-\psi_{j}=$ $\operatorname{Re} f_{i j}$ for some holomorphic function $f_{i j}$ on $U_{i} \cap U_{j}$. Then it is easy to see that we can take $\left\{d f_{i j}\right\}$ as a representative $c$ as above and

$$
\alpha=\left\{\partial \psi_{i}\right\}
$$

defines a connection of type $(1,0)$ of $\gamma$ with $\omega=i / 2 \pi \bar{\partial} \alpha$. If $\omega$ is the first chern form $c_{1}(L, h)$ of some hermitian line bundle $(L, h)$ the unique hermitian connection for $(L, h)$ is a typical such connection.

We now compare the exact sequences (2.5) and (2.14). By the five lemma it is immediately clear that $H^{q}\left(\Sigma_{\gamma}\right)$ is isomorphic to $H^{q}\left(B^{\circ}\right)$. It is important, however, to have a canonical such isomorphism in defining generalized Weil-Petersson metrics later. In the sequel we write $\Sigma$ for $\Sigma_{\gamma}$.

Theorem 2.9. Associated to any connection $\theta$ of type $(1,0)$ of $\gamma$ with curvature $\omega$ as above, there exists a natural isomorphism $\lambda^{q}: H^{q}\left(B^{\circ}\right) \rightarrow H^{q}(\Sigma)$ for each $q \geqq 0$ which extends to an isomorphism of the sequences (2.5) and (2.14), where the other morphisms are all identities.

In particular, if $\omega=c_{1}(L, h)$ for some hermitian line bundle $(L, h)$, then by taking the unique hermitian connection as $\theta$, we have a canonical such isomorphism. More generally, one can show that $\lambda^{1}$ actually is independent of the choice of a connection $\theta$ as above. By composing with the isomorphisms $v^{q}: \mathscr{H}_{B}^{q} \neg H^{q}\left(B^{\circ}\right)$ we get isomorphisms

$$
\mu^{q}: \mathscr{H}_{\mathrm{B}}^{q} \rightarrow H^{q}(\Sigma)
$$


For the proof, first we note that the sequence $(2.14)$ is obtained from the exact sequence of the Dolbeault complexes

$$
0 \longrightarrow \mathbb{D}^{q} \stackrel{l^{q}}{\longrightarrow} \hat{A}^{q} \stackrel{\pi^{q}}{\longrightarrow} A^{q} \longrightarrow 0, \quad q \geqq 0,
$$

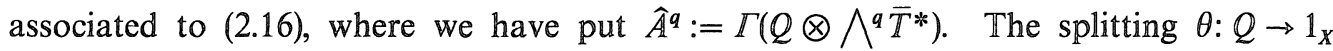
determines naturally a homomorphism $\chi: T \rightarrow Q$ such that $\chi \pi=\mathrm{id}_{T}$ and $\operatorname{Im} \chi=$ $\mathbb{K} e r \theta$. Denote by $\theta^{q}: \widehat{A}^{q} \rightarrow \mathbb{D}^{q}$ and $\chi^{q}: A^{q} \rightarrow \widehat{A}^{q}$ be the induced homomorphisms. Define a $\mathbb{C}$-linear map $j^{0}: B^{0}=\mathbb{C}^{\infty}(X) \rightarrow \widehat{A}^{0}$ by

$$
j^{0}(f)=-l(f)+2 \pi / i \chi\left(\uparrow_{\omega} \bar{\partial}\right)(f),
$$

where $l=\iota^{0}: C^{\infty}(X)=\Gamma\left(1_{X}\right) \rightarrow \widehat{A}^{0}$. We first prove

Proposition 2.10. $\quad \chi^{\circ}: B^{\circ} \rightarrow \hat{A}^{\circ}$ is a homomorphism of complexes.

The essential point is contained in the following:

Lemma 2.11。 $\theta^{q+1} \bar{\partial} \chi^{q}(V)=-i / 2 \pi l_{\omega}^{q}(V)$ for any $V \in A^{q}$.

Proof. The problem is local. So we may assume that $\mathbb{V}$ is of the form $a \otimes b$, where $a$ is a holomorphic vector field and $b$ is a $\mathbb{C}^{\infty}(0, q)$-form on $X$. Then we have

$$
\theta \bar{\partial} \chi(a \otimes b)=\theta \bar{\partial}(\chi(a) \otimes b)=A(\theta \bar{\partial} \chi(a) \otimes b)
$$

since $(\theta \chi(a)) \otimes \bar{\partial} b=0$. This reduces the proof to the case $q=0$ (and $b \equiv 1$ ). Fix a local holomorphic splitting $j: \mathbb{T} \rightarrow \mathbb{Q}$. With respect to $j$ consider $a$ as a holomorphic section of $Q$ and similarly $\theta$ is identified with the $C^{\infty}(1,0)$-form which represents $\theta$ with respect to this splitting. Then we have

$$
\bar{\partial} \chi(a)=\bar{\partial}(a-\theta(a) l)=-\bar{\partial}\left(l_{a} \theta\right) l=-l_{a}(\bar{\partial} \theta) l=-i / 2 \pi\left(l_{a} \omega\right) l
$$

where $l$ is the section 1 of $\mathcal{O}_{X}$ and $l_{a}$ is the contraction with $a$. Hence we get $\theta \bar{\partial} \chi(a)=-i / 2 \pi l_{a} \omega=-i / 2 \pi l_{\omega}(a)$ as desired.

Proof of Proposition 2.10. By Lemma 2.11, if $V \in \mathbb{B}^{q}, q \geqq 1$, then $\bar{\partial} \chi(V) \in \chi\left(\mathbb{B}^{q+1}\right)$. Write $\bar{\partial} \chi(V)=\chi\left(\mathbb{V}^{\prime}\right)$ for some $\mathbb{V}^{\prime} \in A^{q+1}$. Then $V^{\prime}=\pi \chi\left(V^{\prime}\right)=\pi \bar{\partial} \chi(\mathbb{V})=\bar{\partial} \pi \chi(\mathbb{V})=\bar{\partial} \mathbb{V}$. Hence $\bar{\partial} \chi(V)=\chi \bar{\partial}(V)$ as desired. Similarly, again by using Lemma 2.11 we have

$$
\begin{aligned}
\theta \bar{\partial} j^{0}(f) & =-\theta \bar{\partial} l(f)+2 \pi / i(\theta \bar{\partial} \chi)\left(\uparrow_{\omega} \bar{\partial} f\right) \\
& =-\theta l(\bar{\partial} f)+\imath_{\omega}\left(\uparrow_{\omega} \bar{\partial} f\right)=-\bar{\partial} f+\bar{\partial} f=0
\end{aligned}
$$

since $l_{\omega} \uparrow_{\omega}=\downarrow_{\omega} \uparrow_{\omega}=$ identity. It follows that $\bar{\partial} j^{0}(f) \in \operatorname{Im} \chi$. If we write $\bar{\partial} j^{0}(f)=\chi\left(\mathbb{V}^{\prime}\right)$ for some $V^{\prime} \in A^{1}$, we get $V^{\prime}=\bar{\partial} \uparrow_{\omega} \bar{\partial} f=\bar{\partial}_{B} f$ by the same argument as above. Hence $\bar{\partial} j^{0}(f)=\chi\left(\bar{\partial}_{B} f\right)$.

Proof of Theorem 2.9. By Proposition 2.10 $\chi^{q}, q \geqq 1$, and $j^{0}$ induce a homomorphism $\lambda^{q}: H^{q}\left(B^{\circ}\right) \rightarrow H^{q}\left(A^{\circ}\right)=H^{q}(\Sigma)$. Therefore the theorem follows by five lemma if we show that $\lambda^{q}$ fit into the sequence (2.5) and (2.14). In view of the definitions of the coboundary map in (2.5) this follows immediately from Lemma 2.11 and (2.20). 


\section{§3. Families of Polarized Kähler and Hodge Mamifolds}

In this section we introduce the notions of polarized and metrically polarized families of compact Kähler and Hodge manifolds, which will be the basic objects of our study throughout the paper.

First of all, the notions of polarized compact $\mathbb{K a ̈ h l e r ~ m a n i f o l d s ~ a n d ~ f a m i l i e s ~ o f ~ s u c h ~}$ manifolds were introduced in [Fu3] and [Sch3] in slightly different but equivalent formulations. Let $X$ be a compact connected $\mathbb{K} a ̈ h l e r$ manifold. Any Kähler form $\omega$ on $X$, being $d$-closed, determines a de $\mathbb{R}$ ham class $[\omega]$ in $H^{2}(X, \mathbb{R})$. In general such a class $\gamma$ is called a $\mathbb{K a ̈ h l e r}$ class, or a $\mathbb{K}$ ähler polarization, on $X$, and the resulting pair $(X, \gamma)$ is called a polarized (compact) Kähler manifold.

In what follows $f: \mathscr{X} \rightarrow S$ always denotes a proper smooth morphism of reduced complex spaces with connected fibers $X_{s}:=f^{-1}(s)$. In this case we shall call $f$ also a family of compact complex manifolds (over $S$ ). $\mathbb{F}$ or an abelian sheaf $\mathscr{F}$ on $\mathscr{X}$ we write $\left(R^{q} f_{*} \mathscr{F}\right)(S)=\Gamma\left(S, R^{q} f_{*} \mathscr{F}\right)$.

Deffinition 3.1. A polarized family $(f: \mathscr{X} \rightarrow S, \tilde{\gamma})$ of (compact) Kähler manifolds consists of a family of compact complex manifolds $f: \mathscr{X} \rightarrow S$ and an element $\tilde{\gamma} \in\left(R^{2} f_{*} \mathbb{R}\right)(S)$ such that all the restrictions $\gamma_{s}:=\tilde{\gamma} \mid X_{s} \in H^{2}\left(X_{s}, \mathbb{R}\right), s \in S$, are $\mathbb{K a ̈ h l e r}$ classes on $X_{s}$. In this case $\gamma$ is called a polarization of the family $f$. Isomorphisms of two families are defined in an obvious way.

In [Sch3] a Kähler class is considered rather as an element of $H^{1}\left(X, \Omega_{X}^{1}\right)$, and accordingly, a polarization $\gamma$ for a family is considered as an element of $R^{1} f_{*} \Omega_{X / S}^{1}(S)$. Moreover, $f$ is assumed to be Kähler (at least locally with respect to $S$ ) in the following sense: There exist an open covering $\mathscr{U}=\left\{U_{i}\right\}$ of $\mathscr{X}$ and $\mathbb{R}$-valued $C^{\infty}$ functions $p_{i}$ on $U_{i}$ such that $p_{i}-p_{j}$ are pluriharmonic on $U_{i} \cap U_{j}$, and that the resulting relative real $(1,1)$-form $\omega_{\mathscr{X} / S}=i \partial_{\mathscr{X} / S} \bar{\partial}_{\mathscr{X} / S} p_{j}$ restricts to a $\mathbb{K a ̈ h l e r}$ form on each fiber. However, as we shall show in Lemma 3.2 below, in any polarized family $(f: \mathscr{X} \rightarrow S, \tilde{\gamma})$ as in Definition $3.1 f$ is actually a $\mathbb{K a ̈ h l e r}$ morphism in the above sense. Further, the equivalence of the two notions of the polarizations of families follows from the next proposition.

Proposition 3.1. Let $(f: \mathscr{X} \rightarrow S, \tilde{\gamma})$ be a polarized family of Kähler manifolds. Then $\tilde{\gamma}$ induces a section $\gamma_{\mathscr{X} / S}$ of $R^{1} f_{*} \Omega_{\mathscr{X} / S}^{1}$ whose restriction to each fiber is a Kähler class, and vice versa.

Proof. Let $\alpha \in\left(R^{2} f_{*} f^{\circ} \mathcal{O}_{S}\right)(S)$ be the image of $\tilde{\gamma} \in\left(R^{2} f_{*} \mathbb{R}\right)(S)$ by the natural homomorphism $R^{2} f_{*} \mathbb{R} \cong R^{2} f_{*} f^{\circ} \mathbb{R} \rightarrow R^{2} f_{*} f^{\circ} \mathcal{O}_{S}$. Observe then that the sequence

$$
0 \rightarrow R^{1} f_{*} d_{\mathscr{X} / S} \mathcal{O}_{\mathscr{X}} \rightarrow R^{2} f_{*} f^{\circ} \mathcal{O}_{S} \rightarrow R^{2} f_{*} \mathcal{O}_{\mathscr{X}} \rightarrow 0
$$

coming from the short exact sequence $0 \rightarrow f^{\circ} \mathcal{O}_{S} \rightarrow \mathcal{O}_{\mathscr{X}} \rightarrow d_{\mathscr{X} / S} \mathcal{O}_{\mathscr{X}} \rightarrow 0$ is exact with $\mathbb{R}^{1} f_{*} d_{\mathscr{X} / S} \mathcal{O}_{\mathscr{X}}$ locally free (as well as $\mathbb{R}^{q} f_{*} d_{\mathscr{X} / S} \mathcal{O}_{\mathscr{X}}$ for any other $q$ ). Here $\alpha$ is mapped to zero in $\left(R^{2} f_{*} \mathcal{O}_{\mathscr{X}}\right)(S)$ since this is true fiber-wise. Hence $\alpha$ comes from a uniquely determined section of $R^{1} f_{*} d_{\mathscr{X} / S} \mathcal{O}_{\mathscr{X}}$ which gives rise to a desired section $\gamma_{\mathscr{X} / S}$ of $R^{1} f_{*} \Omega_{\mathscr{X} / S}^{1}$.

Suppose conversely that we are given an element $\gamma_{\mathscr{X} / S}$ of $\left(R^{1} f_{*} \Omega_{\mathscr{X} / S}^{1}\right)(S)$ with the property of the proposition. Consider the usual inclusions 


$$
\begin{gathered}
f_{*} \Omega_{\mathscr{X} / S}^{1} \subseteq \mathbb{R}^{1} f_{*} d_{\mathscr{X} / S} \mathcal{O}_{\mathscr{X}} \cong \mathbb{R}^{2} f_{*} f^{\circ} \mathcal{O}_{S} \\
\text { UII } \quad \text { UII UII } \\
\text { (0) } \cong\left(\mathbb{R}^{2} f_{*} \Omega_{\mathscr{X} / S}^{1}\right)_{\mathbb{R}} \cong \mathbb{R}^{2} f_{*} f^{\circ} \mathbb{R}
\end{gathered}
$$

where the second row is induced by the conjugation in $\mathbb{R}^{2} f_{*} f^{\circ} \mathcal{O}_{S}$. Then $\gamma_{\mathscr{X} / S}$ clearly induces $\tilde{\gamma} \in\left(R^{2} f_{*} \mathbb{R}\right)(S)$. One can see easily that the assignments $\gamma \rightarrow \gamma_{\mathscr{X} / S}$ and $\gamma_{\mathscr{X} / S} \rightarrow \tilde{\gamma}$ above are inverses to each other.

Let now $f: \mathscr{X} \rightarrow S$ be a family of compact complex manifolds in general. Then a family $\tilde{\omega}=\left\{\omega_{s}\right\}_{s \in S}$ of $\mathbb{K a ̈ h l e r}$ forms $\omega_{s}$ on the fibers $X_{s}$ is called of class $\mathbb{C}^{\infty}$ if it is induced by a section of $\Phi_{\mathscr{X} / S} \subseteq \mathscr{D}_{\mathscr{X} / S}^{1,1}$ (cf. (1.9)), which is then uniquely determined by $\tilde{\omega}$. We then identify $\tilde{\omega}$ with the corresponding section of $f_{*} \Phi_{\mathscr{X} / \mathbf{S}}$. The induced Kähler classes $\tilde{\gamma}:=\left\{\gamma_{s}\right\} \in H^{2}\left(X_{s}, \mathbb{R}\right)$ are then induced by the image $\delta_{2} \delta_{1}(\tilde{\omega}) \in\left(\mathbb{R}^{2} f_{*} f^{\circ} \mathscr{D}_{S}(\mathbb{R})\right)(S)$ of $\tilde{\omega}$ via the composite map

$$
f_{*} \Phi_{\mathscr{X} / S} \stackrel{\delta_{1}}{\longrightarrow} R^{1} f_{*} \mathscr{P}_{\mathscr{X} / S} \stackrel{\delta_{2}}{\longrightarrow} \mathbb{R}^{2} f_{*} f^{\circ} \mathscr{D}_{\mathbf{S}}(\mathbb{R})
$$

where $\delta_{1}$ and $\delta_{2}$ are the coboundary homomorphisms induced by the sequences $(1.10)$ and (1.8) respectively.

Definition 3.2. A metrically polarized family $(f: \mathscr{X} \rightarrow S, \tilde{\omega})$ of compact Kähler manifolds consists of a family $f: \mathscr{X} \rightarrow S$ of compact complex manifolds and a $\mathbb{C}^{\infty}$ family $\tilde{\omega}=\left\{\omega_{s}\right\}$ of $\mathbb{K a ̈ h l e r}$ forms such that $\tilde{\gamma}:=\delta_{2} \delta_{1}(\tilde{\omega})$ is constant, i.e., $\tilde{\gamma} \in\left(\mathbb{R}^{2} f_{*} \mathbb{R}\right)(S) \subseteq$ $R^{2} f_{*} f^{\circ} \mathscr{D}_{S}(\mathbb{R})(S)$; thus $(f, \tilde{\omega})$ naturally gives rise to a polarized family $(f, \tilde{\gamma})$ of $\mathbb{K a ̈ h l e r}$ manifolds. In this case $\tilde{\omega}$ is called a metric polarization for $f$, or for $(f, \tilde{\gamma})$.

We shall see below that any metric polarization is always induced by a Kähler form on the total space locally with respect to $S$. First we give a definition.

Definition 3.3. Let $(f: \mathscr{X} \rightarrow S, \tilde{\omega})$ be a metrically polarized family as above and $\omega_{\mathscr{X}}$ a locally $\partial \bar{\partial}$-exact real $C^{\infty}(1,1)$-form on $\mathscr{X}$ i.e., a section of $\Phi_{\mathscr{X}}$. We shall call $\omega_{\mathscr{X}}$ an admissible $(1,1)$-form for $(f, \tilde{\omega})$ if its restriction $\omega_{\mathscr{X}} \mid X_{S}$ to each fiber agrees with $\omega_{s}$.

Lemma 3.2. Let $(f: \mathscr{X} \rightarrow S, \tilde{\gamma})$ be a polarized family. Then there exist locally with respect to $S$ a metric polarization $\tilde{\omega}$ for $(f, \tilde{\gamma})$, and an admissible $(1,1)$-form $\omega_{\mathscr{X}}$ for the resulting metrically polarized family $(f, \tilde{\omega})$.

We prove the lemma in a more general form for later use. Let $(f: \mathscr{X} \rightarrow S, \tilde{\gamma})$ be as above. Let $S^{\prime} \subseteq S$ be a closed analytic subset and $\mathscr{X}^{\prime}:=\mathscr{X} \times{ }_{S} S^{\prime}$. Set $\tilde{\gamma}^{\prime}=\tilde{\gamma} \mid \mathscr{X}^{\prime}$ and $f^{\prime}=f \mid \mathscr{X}^{\prime}$. Then $\left(f^{\prime}: \mathscr{X}^{\prime} \rightarrow S^{\prime}, \tilde{\gamma}^{\prime}\right)$ is again a polarized family. Then Lemma 3.2 follows from the special case of the next lemma where $S^{\prime}$ reduces to a single point.

Lemma 3.3. Let $\tilde{\omega}^{\prime}$ be a metric polarization for $\left(f^{\prime}, \tilde{\gamma}^{\prime}\right)$ with an admissible $(1,1)$-form $\omega_{\mathscr{X}}$. Then $\tilde{\omega}^{\prime}$ extends locally with respect to $S$ to a metric polarization $\tilde{\omega}$ for $(f, \tilde{\gamma})$, and $\omega_{\mathscr{X}}$, to an admissible $(1,1)$-form $\omega_{\mathscr{X}}$ for $(f, \tilde{\omega})$.

Proof. We consider the following commutative diagrams with exact rows, considered around an arbitrary point of $S^{\prime}$; 


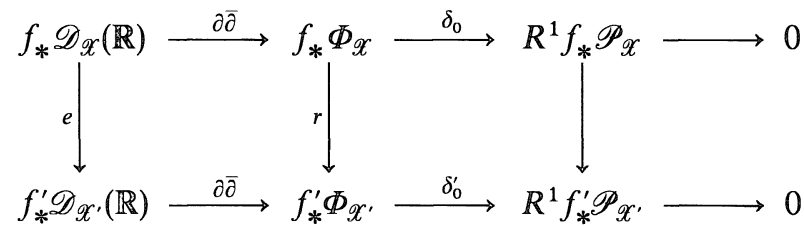

and

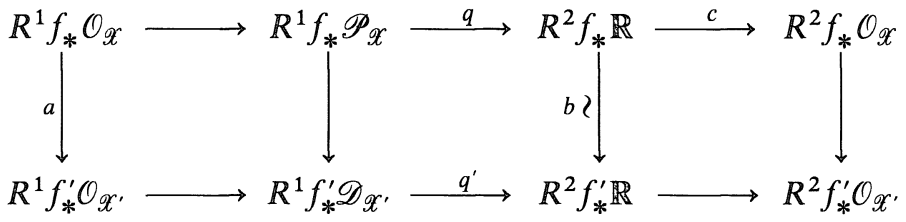

coming from the short exact sequences (1.2) and (1.1) on $\mathscr{X}$ and $\mathscr{X}^{\prime}$. Here, $a$ and $e$ are surjective, and $b$ is isomorphic. (For $a$ we use the cohomological flatness of $\mathcal{O}_{\mathscr{X}}$ with respect to $f$.) Moreover, $\beta:=b^{-1} q^{\prime} \delta_{0}^{\prime}\left(\omega_{X^{\prime}}\right) \in\left(R^{2} f_{*} \mathbb{R}\right)(S)$ is just the given polarization $\tilde{\gamma}$. Therefore, $c(\beta)=0$, and $\beta$ is in the image of $q$. From these, by a simple diagram chase we can find a section $\omega_{\mathscr{X}}$ of $f_{*} \Phi_{\mathscr{X}}$ with $r\left(\omega_{\mathscr{X}}\right)=\omega_{\mathscr{X}}$. Then this $\omega_{\mathscr{X}}$ and the associated metric polarization $\tilde{\omega}$ clearly satisfy the requirement of the lemma.

Proposition 3.4. Let $(f: \mathscr{X} \rightarrow S, \tilde{\omega})$ be a metrically polarized family. Then locally with respect to $S$ there exists always an admissible $(1,1)$-form $\omega_{\mathscr{X}}$ for $(f, \tilde{\omega})$.

We need a lemma.

Lemma 3.5. The homomorphism $\delta_{2}: R^{1} f_{*} \mathscr{P}_{\mathscr{X} / S} \rightarrow R^{2} f_{*} f^{\circ} \mathscr{D}_{S}(\mathbb{R})$ in (3.1) is injective.

Proof. By definition $\delta_{2}$ fits into the exact sequence

$$
R^{1} f_{*} f^{\bullet} \mathscr{D}_{S}(\mathbb{R}) \stackrel{\beta}{\longrightarrow} R^{1} f_{*} \mathcal{O}_{\mathscr{X} / S} \longrightarrow R^{1} f_{*} \mathscr{P}_{\mathscr{X} / S} \stackrel{\delta_{2}}{\longrightarrow} R^{2} f_{*} f^{\bullet} \mathscr{D}_{S}(\mathbb{R}) \text {. }
$$

Hence it suffices to show that $\beta$ is surjective. Note that $R^{1} f_{*} f^{\bullet} \mathscr{D}_{S}(\mathbb{R})=$ $R^{1} f_{*} \mathbb{R} \otimes_{\mathbb{R}} \mathscr{D}_{S}(\mathbb{R})$ is a locally free $\mathscr{D}_{S}(\mathbb{R})$-module, while $R^{1} f_{*} \mathcal{O}_{\mathscr{X}_{/ S}}$ is a locally free $\mathscr{D}_{S^{-}}$ module of rank equal to $\operatorname{dim} H^{1}\left(X_{s}, \mathcal{O}_{X_{s}}\right)$ by $S$ (cf. Lemma 12.6 below). Then the surjectivity of $\beta$ follows from the following commutative diagram

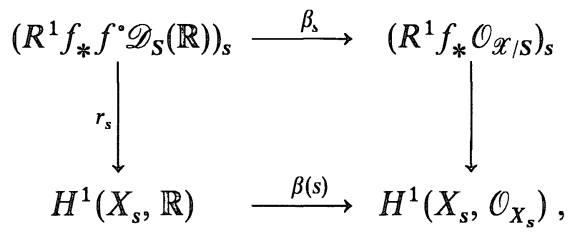

where $s$ is any point, the vertical arrows are the restriction maps, and both $r_{s}$ and $\beta(s)$ are surjective.

Proof of Proposition 3.4. Let $(f, \tilde{\gamma})$ be the induced polarized family. By Lemma 3.2 we can find a metric polarization $\tilde{\omega}^{\prime}$ for $(\tilde{f}, \tilde{\gamma})$ which possesses an admissible $(1,1)-$ form $\omega_{\mathscr{X}}^{\prime}$. Then $\tilde{\omega}-\tilde{\omega}^{\prime} \in\left(f_{*} \Phi_{\mathscr{X} / \mathrm{S}}\right)(S)$ is mapped to zero by $\delta_{1}$ in (3.1) by Lemma 3.5 . Therefore we can find an element $\psi \in \Gamma\left(\mathscr{X}, \mathscr{D}_{\mathscr{X}}(\mathbb{R})\right.$ ) such that $\tilde{\omega}=\tilde{\omega}^{\prime}+i \partial_{\mathscr{X} / S} \bar{\partial}_{\mathscr{X} / S} \psi$ (cf. (3.2) below). We then define $\omega_{\mathscr{X}}=\omega_{\mathscr{X}}^{\prime}+i \partial_{\mathscr{X}} \bar{\partial}_{\mathscr{X}} \psi$, which is clearly admissible for $(f, \tilde{\omega})$. 
Let $(f: \mathscr{X} \rightarrow S, \tilde{\omega})$ be as above. We observe the following commutative diagram with exact rows

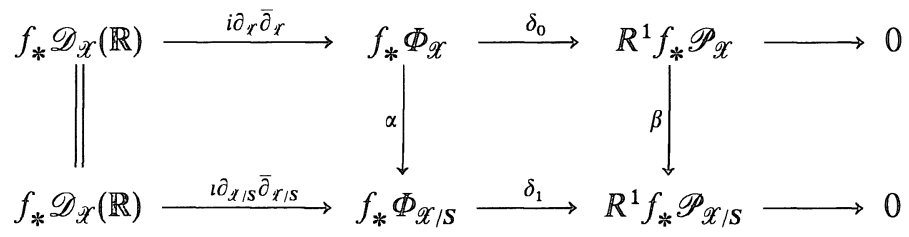

Definnition 3.4. We say that an element $\eta \in\left(\mathbb{R}^{1} f_{*} \mathscr{P}_{\mathscr{P}}\right)(S)$ is compatible with $\tilde{\omega}$ if $\beta(\eta)=\delta_{1}(\tilde{\omega})$. In such a case a triple $(f, \tilde{\omega}, \eta)$ is called a refined metrically polarized family (of Kähler manifolds).

Remark 3.1. The proofs of Lemma 3.2 and Proposition 3.4 show that for any refined metrically polarized family $(f, \tilde{\omega}, \eta)$ we can always find an admissible $(1,1)$-form $\omega_{\mathscr{X}}$ for $(f, \tilde{\omega})$ with $\delta_{0}\left(\omega_{\mathscr{X}}\right)=\eta$ locally with respect to $S$.

We now fix a metrically polarized family $(f: \mathscr{X} \rightarrow S, \tilde{\omega})$.

Defimition 3.5. We say that two admissible $(1,1)$-forms $\omega_{\mathscr{X}}$ and $\omega_{\mathscr{X}}^{\prime}$ on $X$ for $(f, \tilde{\omega})$ are equivalent if the following equivalent conditions are satisfied:

$$
\delta_{0}\left(\omega_{\mathscr{X}}\right)=\delta_{0}\left(\omega_{\mathscr{X}}^{\prime}\right) \quad \text { as a section of } \mathbb{R}^{1} f_{*} \mathscr{P}_{\mathscr{X}}
$$

and

(3.4) we may write $\omega_{\mathscr{X}}^{\prime}=\omega_{\mathscr{X}}+f^{*} \omega_{S}$ for some locally $\partial \bar{\partial}$-exact $(1,1)$-form $\omega_{S}$ on $S$.

The equivalence of (3.3) and (3.4) follows easily from the commutative diagram (3.2). If $\omega_{\mathscr{X}}$ is admissible for $(f, \tilde{\omega})$, then any $(1,1)$-form which is equivalent to it in the sense of (3.4) is again an admissible $(1,1)$-form. In particular, by taking $\omega_{S}$ to be a suitable Kähler form on $S$ we may always take $\omega_{\mathscr{X}}$ to be a Kähler form (locally with respect to $S$ ).

Definition 3.6. We call an admissible $(1,1)$-form $\omega_{\mathscr{X}}$ for $(f, \tilde{\omega})$ normalized if the fiber integral of $\omega_{\mathscr{X}}$, as a $(1,1)$-form on $S$, satisfies the condition

$$
\int_{\mathscr{X} / S} \omega_{\mathscr{X}}^{n+1}=0,
$$

where $n$ is the dimension of the fibers.

Propositiom 3.6. 1) Let $(f, \tilde{\omega})$ be as above. In each equivalence class of admissible $(1,1)$-forms for $(f, \tilde{\omega})$ there exists a unique normalized form $\omega_{\mathscr{X}}$ locally with respect to $S$. 2) For any refined metrically polarized family $(f: \mathscr{X} \rightarrow S, \tilde{\omega}, \eta)$ there exists a unique normalized admissible $(1,1)$-form $\omega_{\mathscr{X}}$ such that $\delta_{0}\left(\omega_{\mathscr{X}}\right)=\eta$. 3) For any element $\eta_{\mathscr{X}} \in$ $H^{1}\left(\mathscr{X}, \mathscr{P}_{\mathscr{X}}\right)$ there exists an admissible $(1,1)$-form $\omega_{\mathscr{X}}$ on $\mathscr{X}$ such that $\delta_{\mathscr{X}}\left(\omega_{\mathscr{X}}\right)=\eta_{\mathscr{X}}$, where $\delta_{\mathscr{X}}: \Gamma\left(\mathscr{X}, \Phi_{\mathscr{X}}\right) \rightarrow H^{1}\left(\mathscr{X}, \mathscr{P}_{\mathscr{X}}\right)$ is the coboundary map coming from (1.2).

Proof. Fix any admissible $(1,1)$-form $\omega_{\mathscr{X}}$ according to Proposition 3.4. From the equation ' $\omega_{\mathscr{X}}=\omega_{\mathscr{X}}+f^{*} \omega_{S}$ we obtain

$$
{ }^{\prime} \omega_{\mathscr{X}}^{n+1} \equiv \omega_{\mathscr{X}}^{n+1}+f^{*} \omega_{s} \wedge \omega_{\mathscr{X}}^{n} \quad \bmod f^{*} \omega_{S} \wedge f^{*} \omega_{S}
$$


By taking the fiber integral we then get

$$
\int_{\mathscr{X} / \mathrm{S}}{ }^{\prime} \omega_{\mathscr{X}}^{n+1}=\int_{\mathscr{X} / \mathrm{S}} \omega_{\mathscr{X}}^{n+1}+C \omega_{S},
$$

where $C=\int_{X_{s}} \omega_{s}^{n}$ is a positive constant which is independent of $s \in S$. Hence, if we set

$$
\omega_{S}=-1 / C \int_{\mathscr{X} / S} \omega_{\mathscr{X}}^{n+1},
$$

then by a theorem of Varouchas (cf. Proposition 1.3) we have $\omega_{S} \in \Gamma\left(S, \Phi_{S}\right)$. Hence, by this choice of $\omega_{S}$ the form ' $\omega_{\mathscr{X}}$ satisfies (3.5), which determines $C$ uniquely. This shows 1 ).

2) follows from the uniqueness in 1) and Remark 3.1 .

3) We observe the following commutative diagram of exact sequences

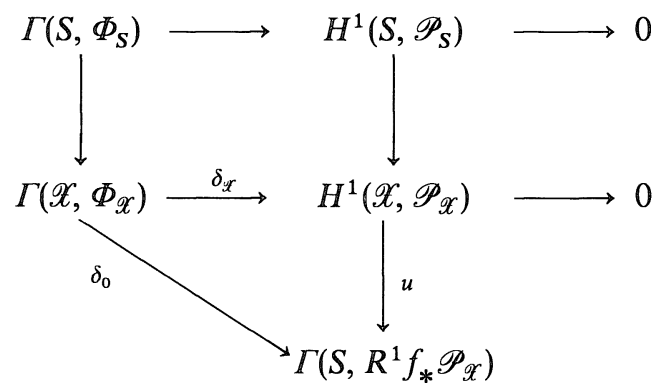

where the right vertical sequence comes from the Leray spectral sequence for $f$, and the horizontal one from (1.2). By 2) we can find an element $\omega_{\mathscr{X}} \in \Gamma\left(\mathscr{X}, \Phi_{\mathscr{X}}\right)$ such that $\delta_{0}\left(\omega_{\mathscr{X}}\right)=u\left(\eta_{\mathscr{X}}\right)$. Then a simple diagram chase shows the existence of an element $\omega_{S} \in \Gamma\left(S, \Phi_{S}\right)$ such that $\delta_{\mathscr{X}}\left(\omega_{\mathscr{X}}+f^{*} \omega_{S}\right)=\eta_{\mathscr{X}}$.

Corollary 3.7. Suppose that the first Betti number $b_{1}\left(X_{s}\right)$ vanishes for any $s \in S$. Then a normalized admissible $(1,1)$-form is unique, and is defined globally on the whole space $X$.

Proof. The exact sequence

$$
\rightarrow R^{1} f_{*} \mathcal{O}_{\mathscr{Y}} \rightarrow R^{1} f_{*} \mathscr{P}_{\mathscr{X}} \rightarrow R^{2} f_{*} \mathbb{R} \rightarrow
$$

shows that $v:\left(R^{1} f_{*} \mathscr{P}_{\mathscr{X}}\right)(S) \rightarrow\left(R^{2} f_{*} \mathbb{R}\right)(S)$ is injective, while if $\tilde{\gamma}$ is the polarization determined by $\tilde{\omega}$, we have $v \delta_{0}\left(\omega_{\mathscr{X}}\right)=\tilde{\gamma}$ for any admissible form $\omega_{\mathscr{X}}$. Thus $\delta_{0}\left(\omega_{\mathscr{Y}}\right)$ is independent of $\omega_{\mathscr{r}}$. The result then follows from Proposition 3.6.

We shall also give another characterization of the equivalence classes of admissible forms.

Lemma 3.8. Let $\omega_{\mathscr{X}}$ and $\omega_{\mathscr{X}}^{\prime}$ be admissible $(1,1)$-forms for $(f, \tilde{\omega})$. Then $T^{H}\left(\omega_{\mathscr{X}}\right)=$ $T^{H}\left(\omega_{\mathscr{X}}^{\prime}\right)$ if and only if $\omega_{\mathscr{X}}$ and $\omega_{\mathscr{X}}^{\prime}$ are equivalent, where $T^{H}\left(\omega_{\mathscr{X}}\right)$ is the horizontal distribution for $f$ associated to $\omega_{\mathscr{X}}$ and $T^{H}\left(\omega_{\mathscr{X}}^{\prime}\right)$ is similar (cf. (1.13)).

Proof. The sufficiency is obvious. The necessity is shown in [B-G-S II; Th.1.7] when $S$ is nonsingular (cf. Remark 3.2 below.) In the general case, let $\omega_{\mathscr{X}}$ and $\omega_{\mathscr{X}}^{\prime}$ 
be two admissible $(1,1)$-forms with $T^{H}\left(\omega_{\mathscr{X}}\right)=\mathbb{T}^{H}\left(\omega_{\mathscr{X}}^{\prime}\right)$, which we may assume to be normalized. Then by the uniqueness in Proposition 3.6 and the above result of $[\mathrm{B}-\mathrm{G}-\mathrm{S}] \omega_{\mathscr{X}}-\omega_{\mathscr{X}}^{\prime}$ must vanish on the smooth locus of $\mathscr{X}$. By Corollary $1.2 \omega_{\mathscr{X}}=\omega_{\mathscr{X}}^{\prime}$ on the whole $\mathscr{X}$.

Remark 3.2. For any $(f, \tilde{\omega})$ and $\omega_{\mathscr{X}}$ as above the resulting triple $\left(f, \omega_{\mathscr{X}}, T^{H}\left(\omega_{\mathscr{X}}\right)\right)$ is exactly the Kähler fibration in the sense of [B-G-S II; Def.1.4]. Lemma 3.8 shows that this notion is equivalent to our notion of refined metrically polarized family as defined above.

For later applications we discuss admissible forms for families of complex tori.

Proposition 3.9. Let $(f: \mathscr{X} \rightarrow S, \tilde{\omega})$ be a metrically polarized family of complex tori such that $\omega_{s}$ are flat Kähler forms on $X_{s}$ for all $s . \quad$ Then, for any admissible $(1,1)$-forms $\omega_{\mathscr{X}}$ and $\omega_{\mathscr{X}}^{\prime}$ for $(f, \tilde{\omega})$, there exists locally with respect to $S$ an automorphism $g$ of $\mathscr{X}$ over $S$ such that $g^{*} \omega_{\mathscr{X}}$ and $\omega_{\mathscr{X}}^{\prime}$ are equivalent.

Proof. The problem is local with respect to $S$. Fixing a holomorphic section to $f$ we put on $\mathscr{X} \rightarrow S$ a natural structure of a relative Lie group over $S$ acting on itself by translations over $S$ (cf. [Fu3; (2.1)]). (Namely, we identify $\mathscr{X} \rightarrow S$ with Aut $_{0} \mathscr{X} / S \rightarrow S$.) We consider the induced action of $\mathscr{X} \rightarrow S$ on the exact sequence

$$
R^{1} f_{*} \mathbb{R} \longrightarrow R^{1} f_{*} \mathcal{O}_{\mathscr{X}} \stackrel{\alpha}{\longrightarrow} \mathbb{R}^{1} f_{*} \mathscr{P}_{\mathscr{X}} \stackrel{\alpha^{\prime}}{\longrightarrow} \mathbb{R}^{2} f_{*} \mathbb{R}
$$

arising from (1.1). Since the action on $\mathbb{R}^{2} f_{*} \mathbb{R}$ is trivial, in view of Definition 3.5 it suffices to show that $\mathscr{X} / S$ acts transitively on the inverse image $P_{\gamma}:=\alpha^{-1}(\tilde{\gamma}) \subseteq$ $\left(R^{1} f_{*} \mathscr{P}_{\mathscr{X}}\right)(S)$ where $\tilde{\gamma}$ is the polarization associated to $\tilde{\omega}$.

We consider the associated infinitesimal action of $f_{*} \Theta_{\mathscr{X} / S}$ where $\Theta_{\mathscr{X} / S}$ is the relative tangent sheaf. The action on $R^{1} f_{*} \mathcal{O}_{\mathscr{X}}$ is given by the coboundary homomorphism $w: f_{*} \Theta_{\mathscr{X} / S} \rightarrow R^{1} f_{*} \mathcal{O}_{\mathscr{X}}$ coming from the relative Atiyah sequence associated to $(f, \tilde{\gamma})$ generalizing (2.13) (cf. (4.2) below). Both $f_{*} \Theta_{\mathscr{X} / S}$ and $R^{1} f_{*} \mathcal{O}_{\mathscr{X}}$ are locally free $\mathcal{O}_{S^{-}}$ modules and on each fiber $w$ induces an isomorphism $H^{0}\left(X_{s}, \Theta_{X_{s}}\right) \neg H^{1}\left(X_{s}, \mathcal{O}_{X_{s}}\right)$; hence $w$ itself is isomorphic. Now $\left(R^{1} f_{*} \mathcal{O}_{\mathscr{X}}\right)(S)$ acts transitively on $P_{\gamma}$ by the exactness of (3.6), and so the orbit map $o:\left(\mathbb{R}^{1} f_{*} \mathcal{O}_{\mathscr{X}}\right)(S) \rightarrow P_{\gamma}, \zeta \rightarrow \alpha(\zeta)+\delta_{0}\left(\omega_{\mathscr{X}}\right)$, is surjective. Thus the action of $\mathscr{X} \rightarrow S$ on $P_{\gamma}$ is transitive.

We now look at families of compact Kähler manifolds which are polarized by chern classes of positive line bundles.

Definition 3.7. 1) A polarized Hodge manifold is a pair $(X, L)$ consisting of a projective manifold and a positive line bundle $L$ on $X$. An isomorphism of two polarized Hodge manifolds $(X, L)$ and $\left(X^{\prime}, L^{\prime}\right)$ is an isomorphism $\psi: X \neg X^{\prime}$ of complex manifolds such that $\psi^{*} L^{\prime} \cong L$.

2) A polarized family of Hodge manifolds $(f: \mathscr{X} \rightarrow S, \mathscr{L})$ consists of a family of projective manifolds $f: \mathscr{X} \rightarrow S$ and a holomorphic line bundle $\mathscr{L}$ on $\mathscr{X}$ whose restrictions $L_{s}:=\mathscr{L} \mid X_{s}$ to fibers are all positive. An isomorphism of two such families $(f$ : $\mathscr{X} \rightarrow S, \mathscr{L})$ and $\left(f^{\prime}: \mathscr{X}^{\prime} \rightarrow S, \mathscr{L}^{\prime}\right)$ is an isomorphism $\psi: \mathscr{X} \neg \mathscr{X}^{\prime}$ of complex spaces over 
$S$ such that $\psi^{*} \mathscr{L}^{\prime}=\mathscr{L} \otimes f^{*} F$ for some holomorphic line bundle $F$ on $S$. This amounts to considering, instead of line bundle itself, the induced section of relative Picard variety.

Remark 3.3. In 1) $(X, L)$ gives rise naturally to a polarized $\mathbb{K a ̈ h l e r ~ m a n i f o l d ~}(X, \gamma)$, where $\gamma=c_{1}(L) \in H^{2}(X, \mathbb{R})$, which is in the image of $H^{2}(X, \mathbb{Z})$. Conversely, any $(X, \gamma)$ with $\gamma \in \operatorname{Im}\left(H^{2}(X, \mathbb{Z}) \rightarrow H^{2}(X, \mathbb{R})\right)$ is induced by some $(X, L)$ as above with $L$ defined up to numerical equivalence; hence a polarized Hodge manifold is just a projective manifold with an (inhomogeneous) polarization in the sense of algebraic geometry (cf. $[\mathrm{P}])$. Similarly, in 2$)(f, \mathscr{L})$ gives rise to a polarized family $(f, \tilde{\omega})$ of Kähler manifolds, where $\omega_{s}=c_{1}\left(L_{s}\right)$.

Definition 3.8. A metrically polarized family of Hodge manifolds is a triple $(f, \tilde{\omega}, \mathscr{L})$, where $(f, \tilde{\omega})$ is a metrically polarized family of Kähler manifolds and $(f, \mathscr{L})$ is a polarized family of Hodge manifolds such that $\omega_{s}$ represents $c_{1}\left(L_{s}\right)$ on $X_{s}$.

Remark 3.4. As in Remark $3.3(f, \tilde{\omega}, \mathscr{L})$ as above gives rise to a refined metrically polarized family $(f, \tilde{\omega}, \eta)$ of Kähler manifolds, where $\eta=\hat{c}(\mathscr{L})$ with the refined chern class $\hat{c}(\mathscr{L})$ identified with its image in $\left(R^{1} f_{*} \mathscr{P}_{\mathscr{X} / S}\right)(S)$ (cf. (1.5)). In fact, one sees from Lemma 3.5 that $\tilde{\omega}$ and $\eta$ are compatible in the sense of Definition 3.4.

Definition 3.9. Let $(f: \mathscr{X} \rightarrow S, \tilde{\omega}, \mathscr{L})$ be a metrically polarized family of Hodge manifolds. We call a hermitian metric $h$ on $\mathscr{L}$ admissible if the first chern form $c_{1}(\mathscr{L}, h)$ is an admissible $(1,1)$-form for $(f, \tilde{\omega})$. If, further, $c_{1}(\mathscr{L}, h)$ is normalized, $h$ is called normalized also.

Proposition 3.10. Let $(f: \mathscr{X} \rightarrow S, \tilde{\omega}, \mathscr{L})$ be a metrically polarized family of Hodge manifolds. Then there exists an admissible (resp. normalized admissible) hermitian metric $h$ on $\mathscr{L}$ (resp. on $\mathscr{L}$ locally with respect to $S$ ). Moreover, the totality of such metrics is given by $h^{\prime}=h \cdot f^{*} e^{p}$ for all $C^{\infty} \mathbb{R}$-valued functions (resp. pluriharmonic functions) $p$ on $S$, where $e^{p}=\exp (p)$.

Proof. By Remark 3.4 and Proposition 3.6 there exists an admissible $(1,1)$-form $\omega_{\mathscr{X}}$ for $(f, \tilde{\omega})$ such that $\delta_{\mathscr{X}}\left(\omega_{\mathscr{X}}\right)=\hat{c}(\mathscr{L})$ in $H^{1}\left(\mathscr{X}, \mathscr{P}_{\mathscr{X}}\right)$. Furthermore, $\omega_{\mathscr{X}}$ can be taken to be normalized if the family is considered locally over $S$. Take now any hermitian metric $h_{o}$ on $\mathscr{L}$. Since $\delta_{\mathscr{X}}\left(c_{1}(\mathscr{L}, h)\right)=\hat{c}(\mathscr{L})$, for some $q \in \Gamma\left(\mathscr{X}, \mathscr{D}_{\mathscr{X}}(\mathbf{R})\right)$ we may write

$$
\omega_{\mathscr{X}}=c_{1}\left(\mathscr{L}, h_{o}\right)-(i / 2 \pi) \partial \bar{\partial} q=c_{1}(\mathscr{L}, h),
$$

where $h=e^{q} h_{o}$, thus proving the first assertion. The above proof further shows that if $c_{1}\left(\mathscr{L}, h^{\prime}\right)$ is admissible for another hermitian metric $h^{\prime}, h=h^{\prime} e^{q^{\prime}}$ for some $q^{\prime} \in$ $\Gamma\left(\mathscr{X}, \mathscr{D}_{\mathscr{X}}(\mathbb{R})\right)$. But since $h / h^{\prime}$ is then on each fiber pluriharmonic, and hence is constant, $q$ is of the form $q=f^{*} p$ for some $p \in \Gamma\left(S, \mathscr{D}_{S}(\mathbb{R})\right)$. If $c_{1}\left(\mathscr{L}, h^{\prime}\right)$ also is normalized, by the uniqueness $q$, and hence $p$ also, must be pluriharmonic.

Corollary 3.11. Locally with respect to $S$ a normalized hermitian metric on $\mathscr{L}$ is determined uniquely up to automorphisms of $\mathscr{L}$ over $\mathscr{X}$. 
Proof. If $h=h^{\prime} \cdot f^{*}\left(e^{p}\right)$ with $p$ pluriharmonic as in the proposition and if $p$ is locally a real part of some $g \in \Gamma\left(S, \mathcal{O}_{S}\right)$, the multiplication by $f^{*} e^{g / 2}$ on the fibers of $\mathscr{L}$ transforms $h^{\prime}$ into $h$.

Remark 3.5. Though $h$ is determined only up to automorphisms of $\mathscr{L}$, the resulting chern form $c_{1}(\mathscr{L}, h)$ is independent of the choice of such an $h$. Hence we always have a global normalized admissible $(1,1)$-form $\omega_{\mathscr{X}}$ which coincides with $c_{1}(\mathscr{L}, h)$ on any open subset on which a normalized admissible hermitian metric is defined. (This is in fact a special case of Proposition 3.6 where $\eta=\hat{c}(\mathbb{L})$.)

Note however that the de $\mathbb{R}$ ham class of $\omega_{\mathscr{X}}$ in $H^{2}(\mathscr{X}, \mathbb{R})$ (cf. Def. 1.8) may in general not coincide with $c_{1}(\mathscr{L})$, or it may even not an integral class. The obstruction for the latter and for the global existence of a normalized hermitian metric is detected as follows.

Proposition 3.12. Let $(f: \mathscr{X} \rightarrow S, \tilde{\omega}, \mathscr{L})$ be as above. Then there exists an element $\xi \in H^{2}\left(S, S^{1}\right)$ whose vanishing is equivalent to the existence of a holomorphic line bundle $F$ on $S$ and a hermitian metric $h$ on $\mathscr{L} \otimes f^{*} F$ such that $c_{1}\left(\mathscr{L} \otimes f^{*} F, h\right)=\omega_{\mathscr{X}}$.

Proof. We observe the following commutative diagram of exact sequences

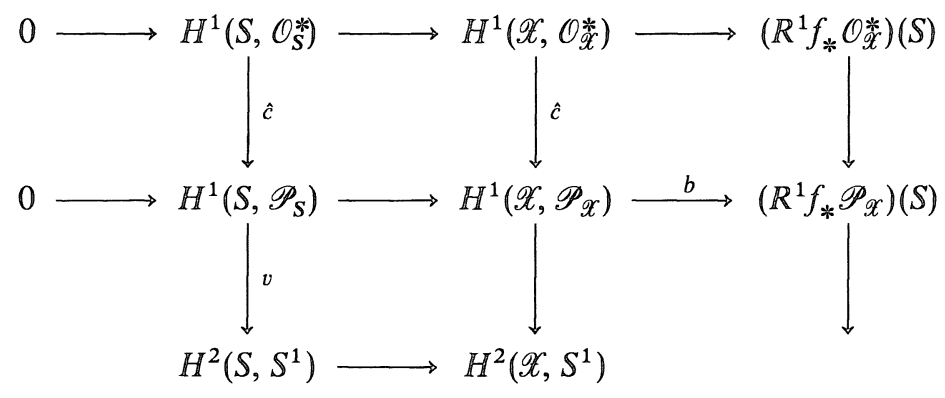

where the horizontal sequences come from the Leray spectral sequences for $f$ and the left and the middle vertical sequences are just the sequences (1.4) for $S$ and $\mathscr{X}$. Let $\eta \in H^{1}\left(\mathscr{X}, \mathscr{P}_{\mathscr{X}}\right)$ be the image of $\omega_{\mathscr{X}} \in \Gamma\left(\mathscr{X}, \Phi_{\mathscr{X}}\right)$. Since $b(\eta)=b(\hat{c}(\mathscr{L}))$, we may consider $\eta-\hat{c}(\mathscr{L}) \in H^{1}\left(S, \mathscr{P}_{S}\right)$. Then we set $\xi=v(\eta-\hat{c}(\mathscr{L}))$. This vanishes if and only if there is a line bundle $F$ on $S$ such that $\eta-\hat{c}(\mathscr{L})=\hat{c}(F)$, or equivalently, $\eta=\hat{c}\left(\mathscr{L} \otimes f^{*} F\right)$ on $\mathscr{X}$. In this case, the proof of Proposition 3.10 shows the existence of an $h$ with $c_{1}\left(\mathscr{L} \otimes f^{*} F, h\right)=\omega_{\mathscr{X}}$ as in the proposition.

\section{\$ 4. Infimitesimal Deformations for Polærized Families}

In this section we shall introduce the Kodaira-Spencer maps associated to the polarized families introduced in the previous section. In the case of a metrically polarized family, we shall see that any admissible $(1,1)$-form gives rise naturally to representatives of the $\mathbb{K}$ odaira-Spencer classes. In the special case of metrics of constant scalar curvature these representatives turn out always to be harmonic, which is closely related with the $\mathbb{K}$ ähler property of generalized Weil-Petersson metrics defined below. 
For a complex space $S$ we shall denote by $\Theta_{S}$ the holomorphic tangent sheaf of $S$. Let $f: \mathscr{X} \rightarrow S$ be a family of compact complex manifolds. Then the associated locally free extension

$$
0 \rightarrow \Theta_{\mathscr{X} / S} \rightarrow \Theta_{\mathscr{X}} \rightarrow f^{*} \Theta_{S} \rightarrow 0
$$

of $f^{*} \Theta_{S}$ by the relative tangent sheaf $\Theta_{\mathscr{X} / S}$ gives rise to the commutative diagram

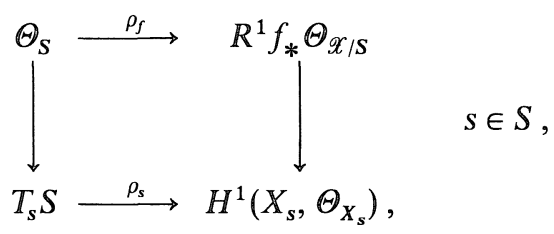

defining the Kodaira-Spencer map $\rho_{s}$ for the family $f$ at $s$, where the vertical arrows are the restriction maps.

Let $\eta$ be any element of $\left(R^{1} f_{*} \mathscr{P}_{\mathscr{X}}\right)(S)$ and assume $S$ to be Stein. The natural homomorphism

$$
\mathscr{P}_{\mathscr{X}} \rightarrow \partial_{\mathscr{X}} \mathscr{P}_{\mathscr{X}} \rightarrow d \mathcal{O}_{\mathscr{X}} \rightarrow \Omega_{\mathscr{X}}^{1}
$$

gives a homomorphism $\zeta: R^{1} f_{*} \mathscr{P}_{\mathscr{X}} \rightarrow R^{1} f_{*} \Omega_{\mathscr{X}}^{1}$. The space $\left(R^{1} f_{*} \Omega_{\mathscr{X}}^{1}\right)(S)=H^{1}\left(\mathscr{X}, \Omega_{\mathscr{X}}^{1}\right)$ classifies the set of isomorphism classes of coherent extensions $0 \rightarrow \mathcal{O}_{\mathscr{X}} \rightarrow \Sigma \rightarrow \Theta_{\mathscr{X}} \rightarrow 0$ of $\Theta_{\mathscr{X}}$ by $\mathcal{O}_{\mathscr{X}}$ such that $\Sigma$ is locally isomorphic to the direct sum $\mathcal{O}_{\mathscr{X}} \oplus \Theta_{\mathscr{X}}$; so $\gamma_{\mathscr{X}}:=\zeta(\eta)$ gives rise to the following commutative diagram of exact sequences of $\mathcal{O}_{\mathscr{X}}$-modules

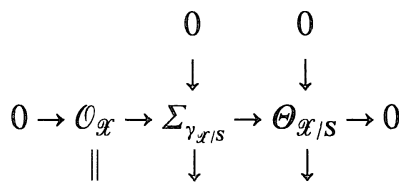

$$
\begin{aligned}
& 0 \rightarrow \mathcal{O}_{\mathscr{X}} \rightarrow \Sigma_{\gamma_{\mathscr{X}}} \rightarrow \Theta_{\mathscr{X}} \rightarrow 0 \\
& \downarrow \downarrow \downarrow \\
& f^{*} \Theta_{S}=f^{*} \Theta_{S} \\
& \downarrow \downarrow \downarrow \\
& 0 \quad 0
\end{aligned}
$$

where the top horizontal sequence is defined by the natural image $\gamma_{\mathscr{X} / S}$ of $\gamma_{\mathscr{X}}$ in the space $H^{1}\left(\mathscr{X}, \Omega_{\mathscr{X} / S}^{1}\right)$, which classifies the set of isomorphism classes of (locally free) extensions of $\Theta_{\mathscr{X} / S}$ by $\mathcal{O}_{\mathscr{X}}$. From the middle vertical sequence we get the following commutative diagram

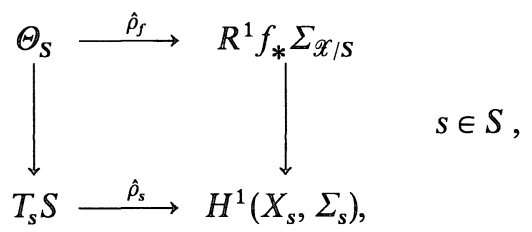

where the vertical maps are the restriction maps and $\Sigma_{s}=\Sigma_{\gamma_{s}}$ (cf. (2.13)). 
Definition 4. 1 . The $\mathbb{C}$-linear map $\hat{\rho}_{s}: T_{s} S \rightarrow H^{1}\left(X_{s}, \Sigma_{s}\right)$ obtained in (4.3) is called the Kodaira-Spencer map at $s$ associated to the family $(f, \eta)$. We say that $(f, \eta)$ is effective if $\hat{\rho}_{s}$ is injective at any $s \in \mathbb{S}$.

From (4.1), (4.2) and (4.3) we get the following commutative diagram with exact rows

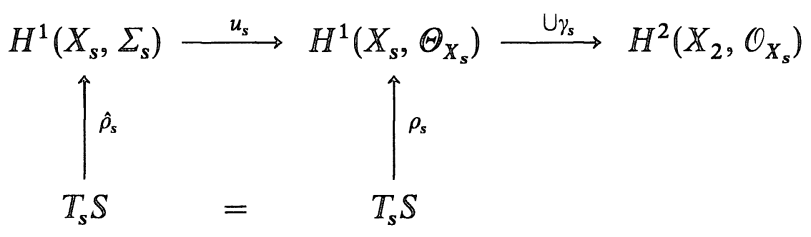

where $\gamma_{s} \in H^{2}\left(X_{s}, \mathbb{R}\right)$ is induced by $\tilde{\gamma}=q(\eta) \in\left(R^{2} f_{*} \mathbb{R}\right)(S)$ (cf. (3.2)). Denote by $H^{1}\left(X_{s}, \Theta_{X_{s}}\right)_{\gamma_{s}}$ the kernel of $\cup \gamma_{s}$.

Lemma 4. 1. Let $(f: \mathscr{X} \rightarrow S, \tilde{\gamma})$ be a polarized family of compact Kähler manifolds. Then for any point $s \in S$ we have $\operatorname{Im} \rho_{s} \subseteq H^{1}\left(X_{s}, \Theta_{X_{s}}\right)_{\gamma_{s}}$.

Proof. Since $\gamma$ is mapped to zero by $\alpha$ in the exact sequence

$$
R^{1} f_{*} \mathscr{P}_{\mathscr{X}} \longrightarrow R^{1} f_{*} \mathbb{R} \stackrel{\alpha}{\longrightarrow} \mathbb{R}^{2} f_{*} \mathcal{O}_{\mathscr{X}},
$$

$\tilde{\gamma}$ can locally always be lifted to an element $\eta$ as above. The result then follows from (4.4).

For any polarized family $(f, \tilde{\gamma})$ as above we call the induced map

$$
\rho_{s}: T_{s} S \rightarrow H^{1}\left(X_{s}, \Theta_{X_{s}}\right)_{\gamma_{s}}
$$

the Kodaira-Spencer map associated to $(f, \tilde{\gamma})$ at $s$. We call $(f, \tilde{\gamma})$ effective if $\rho_{s}$ is injective for all $s$.

Next we consider a polarized family $(f: \mathscr{X} \rightarrow S, \mathscr{L})$ of Hodge manifolds. Let $\eta$ be the natural image of the refined chern class $\hat{c}(\mathscr{L}) \in H^{1}\left(X, \mathscr{P}_{\mathscr{X}}\right)$ in $\left(\mathbb{R}^{1} f_{\mathscr{*}} \mathscr{P}_{\mathscr{X}}\right)(S)$ (cf. (1.5)). In this case we call the Kodaira-Spencer map $\hat{\rho}_{s}$ associated to $(f, \eta)$ the KodairaSpencer map associated to $(f, \mathscr{L})$; and $(f, \mathscr{L})$ is said to be effective if so is $(f, \eta)$.

Note that in this case the diagram (4.2), and hence (4.3) also, can be constructed geometrically from $\mathscr{L}$, e.g., the top horizontal sequence of $(4.2)$ is nothing but the relative Atiyah sequence for the principal $\mathbb{C}^{*}$-bundle $\mathscr{L}^{*}:=\mathscr{L}-0$, which yields on each fiber the Atiyah sequence $(2.13)_{s}$ associated to the principal $\mathbb{C}^{*}$-bundle $L_{s}$ on $X_{s}$. In view of this we write in this case $\Sigma_{\mathscr{L}}=\Sigma_{\gamma_{\mathscr{T}}}$ and $\Sigma_{\mathscr{L}_{/ S}}=\Sigma_{\gamma_{\mathscr{Q} / S}}$ in (4.2).

We next discuss realizations of the above Kodaira-Spencer maps via differential forms. Let $f: \mathscr{X} \rightarrow S$ in general be a family of compact complex manifolds. Let $T^{H}=T^{H} \mathscr{X}$ be any $C^{\infty}$ horizontal distribution for $f(\mathrm{cf} .(\S 1, \mathrm{e}))$. Fix an arbitrary point $o \in S$ and set $X=X_{o}$. Take a $C^{\infty}$ trivialization $\psi: \mathscr{X} \rightarrow X \times S$ over $S$ of $f$ such that

$$
T^{H}\left|X=T^{H} \psi\right| X,
$$

where $T^{H} \psi$ is the (real) horizontal distribution for $f$ defined by the trivialization. (Starting from a given trivialization it is easy to construct one satisfying (4.5).) Now $\psi$ 
induces a $C^{\infty}$ family of almost complex structures on (the underlying $C^{\infty}$ manifold of) $X$ and this is given as usual by a $C^{\infty}$ family $\tilde{\varphi}=\left\{\varphi_{s}\right\}_{s \in S}$ of elements $\varphi_{s}$ of $A^{1}=A^{1}(X)=$ $\Gamma\left(T \otimes T^{*}\right)$ (cf. $\S 2$ ) with $\varphi_{o}=0$. Then for any vector $v \in T_{o} S$ we may speak of the derivative of this family at $o$ with respect to $v$, say $L_{v} \tilde{\varphi}$, as an element of $A^{1}$; the resulting $\mathbb{C}$-linear map $T_{o} S \rightarrow A^{1}, v \rightarrow L_{v} \tilde{\varphi}$, will be denoted by $\xi_{o}$;

$$
\xi_{o}: T_{o} S \rightarrow A^{1} .
$$

This is actually independent of the choice of the above $C^{\infty}$ trivialization $\psi$ and depends only on the restriction $T^{H} \mid X$, which is a complex vector bundle on $X$.

Lemma 4.2. Let $Z^{1}$ be the kernel of $\bar{\partial}$ in $A^{1}$. Then $\xi\left(T_{0} S\right) \subseteq Z^{1}$; moreover, if $p: Z^{1} \rightarrow H^{1}\left(X, \Theta_{X}\right)$ is the natural projection via the Dolbeault isomorphism, we have $\rho_{o}=p \xi_{o}$, where $\rho_{o}: \mathbb{T}_{o} S \rightarrow H^{1}\left(X, \Theta_{X}\right)$ is the Kodaira-Spencer map.

\section{Proof. See e.g. $[\mathrm{Ku}]$.}

Fix $v \in T_{o} S$. Take a local embedding $(S, o) \subset\left(\mathbb{C}^{m}, o\right), \mathbb{C}^{m}=\mathbb{C}^{m}\left(s_{1}, \ldots, s_{m}\right)$, such that $v=\partial / \partial s, s=s_{1}$, at $o$. Choose a local coordinate system of the form $(z, s), z=$ $\left(z_{1}, \ldots, z_{n}\right)$ and $s=\left(s_{1}, \ldots, s_{m}\right)$, on an open subset $\mathscr{U}$ of $\mathscr{X}$ such that $f$ is given by $f(z, s)=s$. Write the horizontal lift $\tilde{v}$ of $v$ as

$$
\tilde{v}=\partial / \partial s+\sum a^{\alpha} \partial / \partial z_{\alpha}
$$

on $U:=X \cap \mathscr{U}$. The following is also well-known:

Lemma A. $\xi_{o}(v)=\bar{\partial}\left(\sum a^{\alpha} \partial / \partial z_{\alpha}\right)=\sum a_{; \bar{\beta}}^{\alpha} \partial / \partial z_{\alpha} \otimes d \bar{z}_{\beta}$ on $U$.

In the above situation if more generally $\tilde{A}=\left\{a_{s}\right\}_{s \in S}$ is a $C^{\infty}$ family of tensor fields $a_{s}$ on $X_{s}$ and $\tilde{A}^{\prime}=\left\{a_{s}^{\prime}\right\}$ the induced family of tensor fields on $X$ via $\psi$, we may consider the tensor field $L_{v} \widetilde{A}^{\prime}$ on $X$. This is also independent of choice of $\psi$ and depends only on $T^{H} \mid X$ and $\widetilde{A}$; so it shall be denoted by $L_{v} \widetilde{A}$. (This is in fact nothing but the relative Lie derivative defined by $T^{H}$ at $o$, at least when $S$ is nonsingular. (See [B-G-S] [Si] e.g.)

Suppose now that there exists a holomorphic line bundle $\mathscr{L}$ on $\mathscr{X}$. Let $\tilde{\phi}: \mathscr{L}^{\times} \rightarrow \mathscr{X}$ be the associated principal $\mathbb{C}^{*}$-bundle. Denote by $\pi: \hat{A}^{1} \rightarrow A^{1}$ be the natural homomorphism as in $\S 2$, where $\hat{A}^{1}=\Gamma\left(X, Q \otimes \bar{T}^{*}\right)$ with respect to the restriction $L:=L_{o}$. Let now $\hat{f}: \mathscr{L} \rightarrow S$ be the natural projection. Suppose that we are given a $\mathbb{C}^{*}$-invariant horizontal subbundle $T^{H} \mathscr{L}$ for $\hat{f}$ such that for any $l \in \mathscr{L}^{\times}, \tilde{\phi}$ induces an isomorphism

$$
T_{l}^{H} \mathscr{L} \cong T_{x}^{H}, \quad x=\tilde{\phi}(l)
$$

Suppose further that $\psi$ is lifted to a trivialization of $\mathbb{C}^{*}$-bundles $\hat{\psi}: \mathscr{L}^{\times} \rightarrow L^{\times} \times S$ such that $T^{H} \mathscr{L}\left|L \cong T^{H} \hat{\psi}\right| L$ in a similar sense as before. In this case the resulting family of almost complex structures on (the underlying $C^{\infty}$ bundle of) $L$ is given by a $C^{\infty}$ family $\hat{\varphi}=\left\{\hat{\varphi}_{s}\right\}$ of elements $\hat{\varphi}_{s}$ of $\hat{A}^{1}$ with $\hat{\varphi}_{o}=0$ (cf. [Gri]). Then, as before, we can take the derivative of this family at $o$ with respect to any $v \in T_{o} S$ and we denote by $\hat{\xi}_{o}: T_{o} S \rightarrow \hat{A}^{1}$ the resulting $\mathbb{C}$-linear map. 
Lemm2 4.4. Let $\hat{Z}^{1}$ be the kernel of $\bar{\partial}$ in $\hat{A}^{1}$. Then: 1) $\hat{\xi}_{o}\left(T_{o} S\right) \subseteq \hat{Z}^{1}$. 2) If $p: \hat{Z}^{1} \rightarrow H^{1}\left(X, \Sigma_{L}\right)$ is the natural projection with respect to the Dolbeault isomorphism, $p \hat{\xi}_{o}=\hat{\rho}_{o}$, where $\hat{\rho}_{o}$ is the Kodaira-Spencer map associated to $(\hat{f}, \mathscr{L})$ at $\left.o .3\right)$ The following diagram is commutative

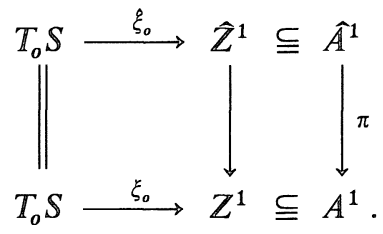

Proof. The proofs of 1) and 2) are the same as that of Lemma 4.2. We only note that the proof of Lemma 4.2 does not require the properness of $f$; so we apply it to $\hat{f}$ and then restrict to the subspace of $\mathbb{C}^{*}$-invariant elements. 3 ) is obvious.

We now consider a metrically polarized family $(f: \mathscr{X} \rightarrow S, \tilde{\omega})$ with a fixed admissible $(1,1)$-form $\omega_{\mathscr{X}}$. We set $T^{H}=T^{H}\left(\omega_{\mathscr{X}}\right)$ as in (1.13) in the above consideration. Then, taking $\tilde{A}$ to be $\tilde{\omega}=\left\{\omega_{s}\right\}$ in the remark after Lemma 4.3 we have a 2 -form $L_{v} \tilde{\omega}$ on $X$.

Lemma 4.5. $L_{v} \tilde{\omega}=0$.

Proof. This was shown in $[\mathbb{B}-G-S \mathbb{I I} ;$ Th. 1.7,d)] when $S$ is nonsingular. The general case can be reduced to this case as follows. The problem is local; we take a local realization of $f$ by a projection as in (1.12) such that $\omega_{\mathscr{X}}$ extends to a $d$-closed $(1,1)$-form $\hat{\omega}_{\mathscr{X}}$ on $W \times D$. Then $\hat{\mathbb{T}}^{H}:=\mathbb{T}^{H}\left(\hat{\omega}_{\mathscr{X}}\right)$ extends $\mathbb{T}^{H}\left(\omega_{\mathscr{X}}\right)$ to a horizontal distribution for $\pi$. Let $\hat{\omega}=\left\{\hat{\omega}_{s}\right\}$ be the family of $\mathbb{K} a ̈ h l e r$ forms induced by $\hat{\omega}_{\mathscr{X}}$ on the fibers of $\pi$. Now applying the above result of $[\mathbb{B}-\mathrm{G}-\mathrm{S}]$ to $\hat{T}^{H}$ and $\hat{\omega}$ we get $L_{v} \hat{\omega}=0$ for any $v \in \mathbb{T}_{o} \mathbb{D}$, where $L_{v}$ is taken with respect to $\pi$. The lemma follows since $L_{v} \hat{\omega}=\mathbb{L}_{v} \tilde{\omega}$ for $v \in \mathbb{T}_{s} S$.

The kernel of $\bar{\partial}$ in $B^{1}=B^{1}(X, \omega), \omega=\omega_{o}$, is denoted by $Z^{1}(B)$ (cf. (2.6)).

Lemma 4.6. If $T^{H}=T^{H}\left(\omega_{\mathscr{X}}\right)$ is as above, then the image of $\xi_{o}$ is contained in $\mathbb{B}^{1}$, and hence in $Z^{1}(B)$.

Proof. With respect to a $\mathbb{C}^{\infty}$ trivialization $\psi$ as above let $\left\{\omega_{s}^{\prime}\right\}$ be the family of 2-forms on $X$ defined by $\tilde{\omega}$. Then $\omega_{s}^{\prime}$ must be isotropic on the graph $\Gamma_{\varphi_{s}} \subseteq \bar{T} \oplus T$ of $\varphi_{s}: \bar{T} \rightarrow T$. Hence we get

$$
0=\omega_{s}^{\prime}\left(u+\varphi_{s}(u), w+\varphi_{s}(w)\right), \quad u, w \in \bar{A}^{0} .
$$

On the other hand, $L_{v} \tilde{\omega}^{\prime}=0$ for any $v \in \mathbb{T}_{o} S$ by Lemma 4.4 ; so by differentiating with respect to $s$ (symbolically) and setting $s=o$ we get

$$
0=\omega\left(\dot{\varphi}_{o}(u), w\right)+\omega\left(u, \dot{\varphi}_{o}(w)\right),
$$

where $\omega=\omega_{o}^{\prime}$. Hence $\dot{\varphi}_{o} \in B^{1}$.

Let $p$ be any local potential function for $\omega_{\mathscr{X}}$. Then in local coordinates we see that in the notation of (4.6)

$$
a_{\bar{\beta}}=\partial \dot{p} / \partial z_{\bar{\beta}}, \quad a_{\bar{\beta}}=g_{\alpha \bar{\beta}} a^{\alpha},
$$


where denotes the derivative $\partial / \partial s$, and $g_{\alpha \bar{\beta}}$ is the metric tensor associated to $\omega_{o}$. Note that $g_{\alpha \bar{\beta}}=\partial^{2} p / \partial z_{\alpha} \partial \bar{z}_{\beta}$ on $U$. (Lemma 4.6 also follows from (4.8).)

Next we treat the case of a metrically polarized family $(f, \tilde{\omega}, \mathscr{L})$ of Hodge manifolds. We fix an admissible hermitian metric $h$ on $\mathscr{L}$ for $(f, \tilde{\omega})$ (locally over $S$ ) and set $\omega_{\mathscr{X}}=c_{1}(\mathscr{L}, h)$. Let $\theta_{\mathscr{L}}:=\partial \log h$ be the hermitian connection of $(\mathscr{L}, h)$, considered as a $\mathbb{C}^{*}$-invariant 1 -form on $\mathscr{L}^{\times}$. Define a $\mathbb{C}^{*}$-invariant horizontal subbundle $T^{H}\left(\theta_{\mathscr{L}}\right)=$ $\cup_{l} T_{l}^{H}\left(\theta_{\mathscr{L}}\right)$ for $\hat{f}$ by

$$
T_{l}^{H}\left(\theta_{\mathscr{L}}\right)=\left\{v \in T_{l}: \theta_{\mathscr{L}}(v)=0, \text { and } \tilde{\phi}_{*}(v) \in T^{H}\left(\omega_{\mathscr{X}}\right)\right\}
$$

This clearly satisfies the condition (4.7).

On the other hand, $\theta_{\mathscr{L}}$ restricts to a hermitian connection $\theta_{s}$ of $\left(L_{s}, h_{s}\right)$ and we apply the relative Lie derivative $\hat{L}_{v}$ for $\hat{f}$ to this family of $C^{\infty} 1$-forms $\tilde{\theta}=\left\{\theta_{s}\right\}$ :

Lemma 4.7. $\hat{L}_{v} \tilde{\theta}=0$ for any $v \in T_{o} S$.

Proof. By the same method as in the proof of Lemma 4.5 we may assume that $S$ is nonsingular. $\hat{L}_{v}$ is then the usual relative Lie derivative associated to $T^{H}\left(\theta_{\mathscr{L}}\right)$. It suffices to show that $\left(\hat{L}_{v} \tilde{\theta}\right)(u)=0$ for any $C^{\infty}$ vertical vector field $u$ on $\mathscr{L}$. Let $\tilde{v}$ be the horizontal lift of $v$ to $\mathscr{L}^{\times}$. Noting that $[\tilde{v}, u]$ is vertical, we have

$$
\begin{aligned}
\left(\hat{L}_{v} \tilde{\theta}\right)(u) & =\tilde{v}(\theta(u))-\theta([\tilde{v}, u])=\tilde{v} \theta(u)-u \theta_{\mathscr{L}}(\tilde{v})-\theta([\tilde{v}, u]) \\
& =\left(d \theta_{\mathscr{L}}\right)_{o}(\tilde{v}, u)=\left(\tilde{\phi}^{*} \omega_{\mathscr{X}}\right)(\tilde{v}, u)=\omega_{\mathscr{X}}\left(\tilde{\phi}_{*} \tilde{v}, \tilde{\phi}_{*} u\right)=0
\end{aligned}
$$

since $\tilde{\phi}_{*} \tilde{v} \in T^{H}\left(\omega_{\mathscr{X}}\right)$ and $\tilde{\phi}_{*} u$ is vertical.

Now consider $\theta=\theta_{o}$ as a homomorphism $\theta: Q \rightarrow 1$ as in Section 2 and let $\theta^{1}$ : $\hat{A}^{1} \rightarrow D^{1}$ be the induced linear map. Recall also the associated linear map $\chi^{1}$ : $\widehat{A}^{1} \rightarrow A^{1}$ with $\pi \chi=\mathrm{id}_{A^{1}}$ and $\operatorname{Im} \chi^{1}=\operatorname{Ker} \theta^{1}$.

Proposition 4.8. Let $\hat{\xi}_{o}: T_{o} \rightarrow \hat{A}^{1}$ be the linear map associated to $T^{H}\left(\theta_{\mathscr{L}}\right)$. Then we have $\hat{\xi}_{o}(v) \in \chi^{1}\left(B^{1}\right)$ for any $v \in T_{o} S$.

Proof. First of all, $\pi \hat{\xi}_{o}(v)=\xi_{o}(v) \in B^{1}$ for any $v$ by Lemma 4.4. On the other hand, since each $\theta_{s}$ is of type $(1,0)$, for any $u \in \widehat{A}^{0}$ we must have

$$
0=\theta_{s}^{\prime}\left(\bar{u}+\hat{\varphi}_{s}(\pi(\bar{u})),\right.
$$

where $\tilde{\theta}^{\prime}=\left\{\theta_{s}^{\prime}\right\}$ is the family of $\mathbb{C}^{*}$-invariant 1 -forms on $L^{\times}$with $\theta_{o}=\theta$ defined by $\hat{\psi}$, and where $\hat{\varphi}_{s}$ is considered a homomorphism $\bar{T} \rightarrow Q$. Then each $\theta_{s}^{\prime}$ is considered a homomorphism $Q \rightarrow 1_{X}$. By Lemma $4.7 \dot{\theta}_{o}^{\prime}=0$ (in the same notation as in the proof of Lemma 4.6). Hence we have

$$
0=\theta^{1}\left(\hat{\varphi}_{o}(\pi(u))\right)=\theta^{1}\left(\hat{\xi}_{o}(v)\right) .
$$

Hence, $\hat{\xi}_{o}(v)=\chi^{1}(u)$ for some $u \in A^{1}$; then $\xi_{o}(v)=\pi \hat{\xi}_{o}(v)=\pi \chi^{1}(u)=u$; so $u \in B^{1}$ as desired.

Lemma 4.9. Let $(f, \tilde{\omega}, \mathscr{L})$ be as above. Then the following diagram is commutative; 


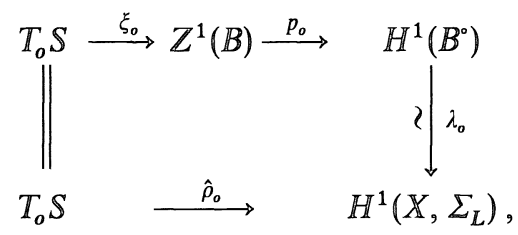

where $p_{o}$ is the natural projection and $\lambda_{o}=\lambda_{o}^{1}$ is as in Theorem 2.9.

Proof. We drop the suffix $o$. By the definition of $\lambda$ we have a $\mathbb{C}$-linear map $\chi: Z^{1}(B) \rightarrow \hat{Z}^{1}:=\left\{\hat{\varphi} \in \hat{A}^{1} ; \bar{\partial} \hat{\varphi}=0\right\}$ such that $\pi \chi$ is the identity and that the following diagram is commutative

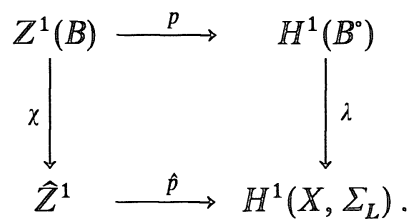

Hence we have by Lemma $4.4 \lambda p \xi=\hat{p} \chi \xi=p \chi \pi \hat{\xi}$. On the other hand, by Proposition 4.8 we have $\chi \pi \hat{\xi}=\hat{\xi}$ since $\chi \pi$ is the identity on $\chi\left(B^{1}\right)$. Thus by Lemma 4.4 we get $\lambda p \xi=\hat{p} \hat{\xi}=\hat{\rho}$.

In order to get an analogue of Lemma 4.9 for a general refined metrically polarized family we shall look at the above construction in the Hodge manifold case in another way. Let $T \mathscr{L}^{\times}$be the tangent linear fiber space of $\mathscr{L}^{\times}$and $\mathscr{Q}_{\mathscr{L}}=T \mathscr{L}^{\times} / \mathbb{C}^{*}$ its quotient with respect to the natural $\mathbb{C}^{*}$ action. Then $Q_{\mathscr{L}}$ is a linear fiber space over $\mathscr{X}$ which fits into the exact sequence

$$
0 \rightarrow 1_{\mathscr{X}} \rightarrow Q_{\mathscr{L}} \rightarrow \mathrm{T} \mathscr{X} \rightarrow 0
$$

of linear fiber spaces; moreover, if we take the sheaf of holomorphic sections in (4.10) we get exactly the middle horizontal sequence of (4.2). Let $\theta_{\mathscr{L}}$ be a connection as above which defines a $C^{\infty}$ splitting of the sequence (4.10).

On the other hand, $\hat{\psi}$ induces a $C^{\infty}$ trivialization of the sequences (4.10); in particular a $C^{\infty}$ trivialization $\psi_{Q}: Q_{\mathscr{L}} \rightarrow S \times Q_{L}$, over $\psi$ which preserves the vector bundle structures and induces the given one $\psi_{*}: T \mathscr{X} \rightarrow S \times T X$ on $T \mathscr{X}$ and the canonical one $1_{\mathscr{X}} \rightarrow S \times 1_{X}$. Then $\psi_{Q}$ induces a $\mathbb{C}^{\infty}$ family of almost complex structures on the vector bundle $Q_{L} \rightarrow X$ which coincides with the standard one on the trivial subbundle $1_{X} \subset Q_{L}$. Such a family is easily seen to be represented by a $C^{\infty}$ family of elements of $\hat{A}^{1}$ and can be identified with the above family $\hat{\varphi}$ (cf. [Gri]).

Now we consider a general refined polarized family $(f: \mathscr{X} \rightarrow S, \tilde{\omega}, \eta)$ of $\mathbb{K a ̈ h l e r}$ manifolds. Let $\omega_{\mathscr{X}}$ be an admissible $(1,1)$-form with $\delta_{1}\left(\omega_{\mathscr{X}}\right)=\eta$ and $\psi: \mathscr{X} \Im S \times X$ a trivialization with $T^{H} \psi\left|X=T^{H}\left(\omega_{\mathscr{X}}\right)\right| X$ as before. In this case we also have the linear fiber space $Q_{\eta} \rightarrow \mathscr{X}$ and the corresponding exact sequence (4.10) which gives rise to the middle horizontal sequence of (4.2). (Note that the situation are the same as in the Hodge case at least locally with respect to $\mathscr{X}$.) Then a connection $\theta_{\mathscr{X}}$ of type $(1,0)$ with curvature $\omega_{\mathscr{X}}$, defined in the same way as (2.15), defines a $C^{\infty}$ splitting of (4.10). Then we set $T^{H} Q:=T^{H}\left(\theta_{\mathscr{X}}\right)=\operatorname{Ker} \theta_{\mathscr{X}} \subseteq Q_{\eta}$. We take a $C^{\infty}$ trivialization $\hat{\psi}$ of (4.10) lifting $\psi$ and with the same property as above such that $T^{H} \psi_{Q}\left|X=T^{H} Q\right| X$, where $\psi_{Q}$ : 
$Q_{\eta} \rightarrow S \times Q, Q:=Q_{\eta} \mid X$, is the induced $C^{\infty}$ trivialization. Then $\psi_{Q}$ induces a $C^{\infty}$ family of complex structures on the fibers of $Q \rightarrow X$ which induces the standard one on $1_{X} \hookrightarrow Q$; this family in turn is represented by a $C^{\infty}$ family of elements of $\hat{A}^{1}$; $\hat{\varphi}=\left\{\hat{\varphi}_{s}\right\}$. Once such a family is obtained, just as above we get a linear map $\hat{\xi}_{o}: T_{o} S \rightarrow$ $\hat{A}^{1}$ depending only on the given $T^{H} Q$, which reduces to the previous one when $(f, \tilde{\omega}, \eta)$ comes from a family of Hodge manifolds. Then we get:

Lemma 4.10. The diagram (4.9) is commutative also in the general case.

Proof. If we show that $\operatorname{Im} \hat{\xi}_{0} \subseteq \chi^{1}\left(B^{1}\right)$, the rest of the proof is the same as in Lemma 4.9. The proof of Proposition 4.8 shows that the assertion follows from the equation $\dot{\theta}_{o}^{\prime}=0$, where $\tilde{\theta}^{\prime}=\left\{\theta_{s}^{\prime}\right\}$ is the $C^{\infty}$ family of connections $\theta_{s}^{\prime}: Q \rightarrow 1_{X}$ defined by $\theta_{\mathscr{X}}$ and $\psi_{Q}$. In fact, the latter follows from Lemma 4.7 since the assertion is local with respect to $\mathscr{X}$ and hence we may assume that we are in the line bundle case.

In the cases of families of Kähler manifolds with constant scalar curvature the following phenomenon occurs which is basic for the Kähler property of the generalized Weil-Petersson metric below.

Theorem 4.11. Let $(f: \mathscr{X} \rightarrow S, \tilde{\omega})$ be a metrically polarized family of Kähler manifolds and $\omega_{\mathscr{X}}$ an admissible $(1,1)$-form for $(f, \tilde{\omega})$. Let $\xi_{s}: T_{s} S \rightarrow Z^{1}(B)$ be the linear map associated to $\left(f, \omega_{\mathscr{X}}\right)$. Then if $\omega_{s}$ is of constant scalar curvature for an $s$, we have $\xi_{s}\left(T_{s} S\right) \subseteq \mathscr{H}_{s}^{1}$, where $\mathscr{H}_{s}^{1}=\mathscr{H}_{B_{s}}^{1}$ is the space of harmonic forms in the complex $B_{s}^{1}:=$ $B^{1}\left(X_{s}, \omega_{s}\right)$.

Proof. Fix any point $o \in S$ and any element $v \in T_{o} S$. We have to show that $\bar{\partial}_{B}^{*} \xi_{o}(v)=0$, or by Lemma 4.3 and (2.9), to show that $a_{\bar{\delta} ; \bar{\beta}} \bar{\beta}^{\bar{\delta} \delta}=0$ in the notation of the lemma. We use the summation convention:

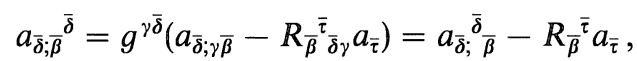

where $R_{\bar{\beta}} \overline{\bar{t}}_{\bar{\delta} \gamma}$ and $R_{\alpha \bar{\beta}}$ denote the curvature, and the Ricci, tensors respectively. All $\omega_{s}$ are of constant scalar curvature $R$, which is independent of the parameter by our polarization assumption. In view of (4.9) settirg $g=\operatorname{det}\left(g_{\alpha \bar{\beta}}\right)$ we get

$$
\left(R_{\alpha \bar{\beta}}\right)^{\circ}=-(\log g)_{; \bar{\beta} \alpha}^{\circ}=-g^{\gamma \bar{\delta}}\left(g_{\gamma \bar{\delta}}\right)_{; \bar{\beta} \alpha}^{\circ}=a_{\bar{\delta} ; \bar{\beta} \alpha}^{\bar{\delta}} .
$$

Hence by (4.12) we have

$$
\begin{aligned}
& 0=\dot{R}=g^{\alpha \bar{\beta}}\left(R_{\alpha \bar{\beta}}\right)^{\bullet}-R^{\alpha \bar{\beta}}\left(g_{\alpha \bar{\beta}}\right)^{\bullet}=-a_{\bar{\delta} ; \bar{\beta}} \bar{\delta}_{\bar{\beta}} \bar{\beta}+R_{\alpha}^{\bar{\beta}} a_{\bar{\beta} ; \alpha} \\
& =a_{\bar{\delta} ; \bar{\beta}} \bar{\beta} \bar{\delta} \text {. }
\end{aligned}
$$

(The last equality holds since the scalar curvature is constant on the fiber.)

Remark 4.1. The above formula (4.13) is a special case of a formula for the variation of the scalar curvature in Riemannian geometry (cf. [B-E]).

In the case of a family of Kähler-Einstein manifolds with nonvanishing Ricci curvature we have a distinguished admissible $(1,1)$-form for $(f, \tilde{\omega})$. First of all, $\tilde{\omega}$ 
induces a hermitian metric, say $h$, on the relative anti-canonical bundle $\mathbb{K}_{\mathscr{X} / S}^{-1}$ and then $\omega_{\mathscr{X}}:=r c_{1}\left(K_{\mathscr{X} / S}^{-1}, h\right)$ is the admissible $(1,1)$-form mentioned above, where $\omega_{s} \in r c_{1}\left(X_{s}\right)$.

Proposition 418. Let $(f: \mathscr{X} \rightarrow \mathbb{S}, \tilde{\omega})$ be a family of $\mathbb{K}$ ähler-Einstein manifolds, and $\omega_{\mathscr{X}}$ the distinguished admissible $(1,1)$-form defined above. Then we have $\xi_{s}\left(\mathbb{T}_{s} S\right) \subseteq \mathscr{H}_{A_{s}}^{1}$ in the same notation as in the previous theorem, where $\mathscr{H}_{\boldsymbol{A}_{\boldsymbol{s}}}^{1}$ is the space of vector valued harmonic 1-forms on $X_{s}$.

Proof. In the same notations as in the previous proof we have to show that $a_{\bar{\delta} ; \bar{\beta}}^{\bar{\delta}}=0 . \quad$ By (4.12) and the $\mathbb{K}$ ähler-Einstein condition, we have

$$
a_{\bar{\delta} ; \bar{\beta}}^{\bar{\delta}}=a_{\bar{\delta} ; \bar{\beta}}^{\bar{\delta}_{\bar{\beta}}}-2 \pi / r a_{\bar{\beta}} .
$$

On the other hand, from (4.9) and the condition

$$
g_{\alpha \bar{\beta}}=r / 2 \pi \partial^{2} \log g / \partial z_{\alpha} \partial \bar{z}_{\beta} .
$$

we have $2 \pi / r a_{\bar{\beta}}=-\partial(\log g)^{\circ} / \partial \bar{z}_{\beta}$, and $a_{\bar{\delta} ;}^{\bar{\delta}}=-g^{\gamma \bar{\beta}} \dot{g}_{\bar{\gamma} \bar{\beta}}=-(\log g)^{\circ}$. From this and (4.14) the desired equality follows.

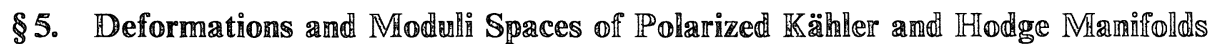

In this section we shall review basic structures of the local and global moduli spaces of polarized Kähler and Hodge manifolds, with special regard to their natural complex $V$-structures in the global case. We also study the structure of the natural morphism between these two moduli spaces.

We start with the Kähler case. In this case, the relevant results were shown in [Fu3] and [Sch2,3]. We review the basic construction rather in detail since the construction of the related moduli spaces treated in this paper follows more or less the same pattern.

In accordance with the previous sections, for simplicity, the base spaces of deformations of complex manifolds are assumed to be reduced.

Definition 5.1. Let $(X, \gamma)$ be a polarized compact $\mathbb{K}$ ähler manifold and $S=(S, o)$ a (reduced) complex space with a distinguished point $o \in \mathbb{S}$. Then a deformation of $(\mathbb{X}, \gamma)$ over $S$ consists of a polarized family $(f: \mathscr{X} \rightarrow S, \tilde{\gamma})$ of compact $\mathbb{K}$ ähler manifolds together with an isomorphisms $(X, \gamma) \rightarrow\left(X_{o}, \gamma_{o}\right)$, where $\gamma_{o}$ is the restriction of $\tilde{\gamma}$ to the fiber $\mathbb{X}_{o}$.

We get as usual the notions of isomorphic deformations, pull-backs of deformations, and of complete, versal (effective + complete), and universal deformations.

Theorem 5.1. Let $(X, \gamma)$ be a polarized compact $\mathbb{K a ̈ h l e r}$ manifold. Then $(\mathbb{X}, \gamma)$ posesses a versal deformation which induces complete deformations of all nearby fibers.

See [Fu3; Prop. 8, Remark 9] [Sch 3; 1.6]. A versal deformation is also called a Kuranishi family and its base space $S=S(X, \gamma)$ a Kuranishi space of $(X, \gamma)$.

Remark 5.1. By the Kodaira-Spencer map $\rho_{o}: \mathbb{T}_{o} S \rightarrow H^{1}\left(X, \Theta_{X}\right)_{\gamma}$ the Zariski tangent space $T_{o} S$ of $S$ at the reference point $o$ is naturally identified with a complex 
subspace of $H^{1}\left(X, \Theta_{X}\right)_{\gamma}$ (cf. Lemma 4.1). If we consider also Kuranishi families with non-reduced base spaces as in [Fu3], $T_{o} S$ actually coincides with $H^{1}\left(X, \Theta_{X}\right)_{\gamma}$.

We shall recall that a $\mathbb{K}$ uranishi family is actually universal under a certain condition on the automorphism group Aut $X$ of $X$. We first explain the condition. Let $A=\operatorname{Alb} X$ be the Albanese variety of $X$ and $J$ : Aut $X \rightarrow$ Aut $A$ be the natural homomorphism (Jacobi homomorphism), where Aut $_{o} X$ denotes the identity component of Aut $X$. Let $L(X)$ be the identity component of the kernel of $J$. Then $L(X)$ has the natural structure of a linear algebraic group and the quotient $\left(\right.$ Aut $\left._{o} X\right) / L(X)$ is a complex torus (cf. [Fu1]). (We call $L(X)$ the linear part of $\mathrm{Aut}_{o} X$.) Hence, the following conditions are equivalent: 1) Aut $t_{o}$ is a complex torus (of dimension $\geqq 0$ ), and 2$) L(X)$ reduces to the identity.

Definnition 5.2. A compact $\mathbb{K}$ ähler manifold is said to be in (the class) $\mathscr{A}$ if $X$ satisfies the above equivalent conditions.

As follows from the definition, in the Lie algebra of all holomorphic vector fields on $X$ the Lie algebra $l(X)$ of $L(X)$ is characterized as the space of annihilators of holomorphic 1-forms. Furthermore, given a Kähler metric $g$ on $X$ it admits the following identification;

$$
l(X)=\left\{i \uparrow_{g} \bar{\partial} f=i \sum f^{\alpha} ; \partial / \partial z^{\alpha} ; f \in \mathscr{H}_{B}^{0}\right\} .
$$

$($ See $[\mathbb{K} ; 4.4]$.)

Now we come back to deformations. Let $(X, \gamma)$ be any polarized compact Kähler manifold and $(f: \mathscr{X} \rightarrow S, \tilde{\gamma})$ a deformation of $(X, \gamma)$ over $S=(S, o)$. Then we have:

Proposition 5.2. Suppose that $X$ is in $\mathscr{A}$. Then in a small neighborhood of o, $X_{s}$ are all in $\mathscr{A}$, and the dimension $\operatorname{dim} A u t\left(X_{s}, \gamma_{s}\right)\left(=\operatorname{dim}\right.$ Aut $\left.X_{s}\right)$ is independent of $s$; moreover, $\mathrm{Aut}_{o} \mathscr{X} / S \rightarrow S$ is smooth.

Here, Aut $\mathscr{x} / S$ denotes the component of the identity section of the relative Lie group Aut $\mathscr{X} / S$ of relative automorphism of $\mathscr{X}$ over $S$. See [Fu3; 2.4 and Prop. 2] for the first statement, and $[\mathrm{Fu} 3 ; \S 4]$ and $[\mathrm{Sch} 3 ; \S 1]$ for the rest. From this, together with a general principle of deformation theory one gets:

Proposition 5.3. If $X$ is in $\mathscr{A}$, a Kuranishi family of $(X, \gamma)$ is universal, and gives also universal deformations of all nearby fibers.

See [Fu3; Prop.8, Prop.9] and [Sch3; 1.8] for the proof. As a formal consequence of Proposition 5.3 we get a natural action of Aut $(X, \gamma)$ on a Kuranishi space $S=$ $S(X, \gamma)$, which by Proposition 5.2 factors through the finite quotient group $G=$ $G(X, \gamma):=$ Aut $(X, \gamma) /$ Aut $_{o} X$ (cf. [Fu3; 5.3] [Sch2; (3.4), Sch3; 1.9]). However, S/G does not necessarily classify the isomorphism classes of corresponding polarized manifolds $\left(X_{s}, \gamma_{s}\right)$. Therefore, in order to get the moduli space in the analytic category, we have to consider more restrictive classes than $\mathscr{A}$.

First, recall that for any polarized family $\mathscr{X}=(\mathscr{X} \rightarrow S, \tilde{\gamma})$ and $\underline{X}^{\prime}=\left(\mathscr{X}^{\prime} \rightarrow S, \tilde{\gamma}^{\prime}\right)$ of Kähler manifolds the functor $\underline{I s o m}_{S}\left(\mathscr{X}, \underline{X}^{\prime}\right):(\mathrm{An} / S) \rightarrow($ Sets $)$ which assigns to a 
space $T \rightarrow S$ the set of isomorphisms between pull-backs of $\mathscr{X}$ and $\underline{X}^{\prime}$ to $T$ is representable by a complex space $\operatorname{Isom}_{S}\left(\mathscr{X}, \underline{X}^{\prime}\right) \rightarrow S$. Here, and in what follows, $(\mathbb{A} / S)$ denotes the category of complex spaces over $S$.

Now we consider in general a class $\mathscr{K}$ of polarized compact $\mathbb{K a ̈ h l e r}$ manifolds $(X, \gamma)$ such that 1$) \mathscr{K}$ is closed under local deformations and 2$)$ the following condition $(B)$ is satisfied:

(B) For any polarized families $\mathscr{X}$ and $\underline{X}^{\prime}$ as above with $X_{s}$ and $X_{s}^{\prime}$ all in $\mathscr{K}$, $\operatorname{Isom}_{S}\left(\mathscr{X}, \underline{X}^{\prime}\right)$ is proper over $S$.

In particular, $\mathscr{K}$ is a subclass of $\mathscr{A}$.

Then, as almost formal consequences of the above two conditions we get the following:

(5.2) If $(X, \gamma) \in \mathscr{K}$, then for any points $r$ and $s$ of a $\mathbb{K}$ uranishi space $S(X, \gamma),\left(X_{r}, \gamma_{r}\right)$ and $\left(X_{s}, \gamma_{s}\right)$ are isomorphic if and only if $s=g(r)$ for some $g \in G(\mathbb{X}, \gamma)$. (See [Fu3; 6.2] $[$ Sch3; 1.9] and $[\operatorname{Sch} 2 ; \S 3]$.)

(5.3) In the notations of $(\mathbb{B})$ if a sequence of points $s_{v} \in \mathbb{S}$ converges to a point $t \in S$, and if $\left(X_{s_{v}}, \gamma_{s_{v}}\right)$ and $\left(X_{s_{v}}^{\prime}, \gamma_{s_{v}}^{\prime}\right)$ are isomorphic, then $\left(X_{t}, \gamma_{t}\right) \cong\left(X_{t}^{\prime}, \gamma_{t}^{\prime}\right)$. (See [Fu3; Prop.10] [Sch2; (3.4), Sch3; (1.9)]).

As a typical example of $\mathscr{K}$ we can take the class of nonuniruled manifolds (cf. [Fu3]):

(5.4) The class of non-uniruled manifolds are closed under deformations and satisfies the condition $(\mathbb{B})$.

With (5.2), (5.3) and (5.4) in hand, it is standard to construct the global moduli space of non-uniruled $\mathbb{K a ̈ h l e r ~ m a n i f o l d s . ~ F i r s t , ~ w e ~ f i x ~ a n ~ u n d e r l y i n g ~} C^{\infty}$ structure $(M, \alpha)$ consisting of a compact connected $C^{\infty}$ manifold $M$ and a class $\alpha \in H^{2}(\mathbb{M}, \mathbb{R})$. Denote by $\mathfrak{M}_{0}$ the set of isomorphism classes of polarized $\mathbb{K a ̈ h l e r}$ manifold $(\mathbb{X}, \gamma)$ which are diffeomorphic to $(\mathbb{M}, \alpha)$, i.e., there exists a diffeomorphism $\varphi: X \neg \mathbb{M}$ such that $\varphi^{*} \alpha=\gamma$. We also denote by $\mathfrak{D P}^{\prime}=\mathfrak{M}_{\alpha}$ the subset of $\mathfrak{M R}_{0}$ consisting of the classes of non-uniruled manifolds. Since the underlying $C^{\infty}$ structure is invariant under (polarized) deformations, for any point of $\mathfrak{M}$ represented by a polarized $\mathbb{K}$ ähler manifold $(X, \gamma)$ we have the natural map $\pi: S(X, \gamma) \rightarrow \mathfrak{M}$ which induces by (5.2) a bijection $S(X, \gamma) / G(X, \gamma) \Im U(X, \gamma):=\pi(S(X, \gamma)) \subseteq \mathfrak{D}$. Then from (5.2)-(5.4) we obtain the following theorem, which is the main result of [Fu3] and [Sch3].

Theorem 5.4. The set $\mathfrak{M}$ carries a natural structure of a Hausdorff complex space with at most countably many connected components. It also carries a natural structure of a complex $V$-structure induced by the collection $\{S(X, \gamma) \rightarrow U(X, \gamma) ; G(X, \gamma)\}$ of all possible such pairs.

See $[\mathrm{Fu}-\mathrm{Sch}]$ for the countability of the components of $\mathfrak{M}$. For the last assertion we assume more precisely that besides having all the properties mentioned so far each $S(X, \gamma)$ is connected and simply connected. Then the last assertion follows from the above description of the local structure of $\mathfrak{M A}$ and the definition of $V$-structure (Def. 1.3), in view of the next lemma.

Lemman 5.5. Let $S=S(X, \gamma)$ and $S^{\prime}=S\left(X^{\prime}, \gamma^{\prime}\right)$ be two Kuranishi spaces satisfying the above conditions such that $U(X, \gamma) \subseteq \mathbb{U}\left(X^{\prime}, \gamma^{\prime}\right)$. Then there exists an embedding 
$\tau: S \rightarrow S^{\prime}$ over the above inclusion which lifts to a morphism $\tilde{\tau}: \underline{\mathscr{X}} \rightarrow \underline{\mathscr{X}}^{\prime}$ of the corresponding universal families.

We first recall some general fact. Let $T=S \times S$ and $p_{i}: T \rightarrow S, i=1,2$, be the two projections. Let $\mathscr{X}_{i}=\left(\mathscr{X}_{i} \rightarrow T, \tilde{\omega}_{i}\right)$ be the pull-backs of the universal family by $p_{i}$. Let $\Gamma_{g}$ be the graph of the action of an element $g$ of $G=G(X, \gamma)$ on $S$. Consider the morphism $I:=\operatorname{Isom}_{T}\left(\mathscr{X}_{1}, \mathscr{X}_{2}\right) \rightarrow T$ with its image denoted by $R$. Then by (5.2) $R$ is the union of $\Gamma_{g}$. In this case, if $I \rightarrow \widetilde{R} \rightarrow \mathbb{R}$ is the Stein factorization, we have

$$
\widetilde{R}=\coprod_{g} \Gamma_{g} \quad \text { (disjoint union) } .
$$

More precisely, let $q$ : Aut $(X, \gamma) \rightarrow G$ be the natural projection, and $I_{g}$ the connected component of $I$ containing $q^{-1}(g)$ with respect to the identification $I_{(o, o)}=\operatorname{Aut}(X, \gamma)$. Then $I$ is a disjoint union $I=\prod_{g} I_{g}$ and $I_{g} \rightarrow \Gamma_{g}$ is smooth and admits a holomorphic section.

Proof of Lemma 5.5. Pulling back both universal families to $V:=S \times S^{\prime}$ by the natural projections, we get families $\underline{\hat{X}}$ and $\underline{\hat{X}}^{\prime}$. Let $\hat{I}=$ $\operatorname{Isom}_{V}\left(\hat{\mathscr{X}}, \underline{\mathscr{X}}^{\prime}\right)$ and $\hat{I} \stackrel{r}{\rightarrow} \hat{R} \rightarrow V$ the Stein factorization. Since $\hat{I} \rightarrow \widehat{R}$ is locally isomorphic to $I \rightarrow \widetilde{R}$ by the local universality of both families, by (5.5) we see that the natural projection $\widehat{R} \rightarrow S$ is an unramified covering. Since $S$ is connected and simply connected, each connected component $\widehat{R}_{v}$ of $\widehat{R}$ is mapped isomorphically to $S$. This shows the existence of $\tau$ as in the lemma. The last assertion then follows from the existence of holomorphic section to $r^{-1}\left(\widehat{R}_{v}\right) \rightarrow \widehat{R}_{v}$.

$\mathfrak{M}$ with the complex structure as in the theorem is called the moduli space of polarized non-uniruled compact Kähler manifolds (with underlying $C^{\infty}$ structures $(M, \alpha)$ ).

We next consider the moduli problem for polarized Hodge manifolds in parallel with the Kähler case. First of all, starting from Definition 3.7, we get as in Definition 5.1 notions concerning deformations of (polarized) Hodge manifolds such as (universal) deformation of Hodge manifolds, pull-backs of such deformations etc. The following result is well-known (cf. [Gri; 3.2]).

Theorem 5.6. Any polarized Hodge manifold $(X, L)$ possesses a versal deformation which induces a complete deformation of all nearby fibers.

A versal deformation of $(X, L)$ in the theorem is called a (reduced) Kuranishi family of $(X, L)$ and its base space $S=S(X, L)$ a (reduced) Kuranishi space of $(X, L)$.

Remark 5.2. By the Kodaira-Spencer map $\hat{\rho}_{o}: T_{o} S \rightarrow H^{1}\left(X, \Sigma_{L}\right)$ the Zariski tangent space $T_{o} S$ of $S$ at the reference point $o$ is naturally identified with a complex subspace of $H^{1}\left(X, \Sigma_{L}\right)$. If we consider also Kuranishi families with nonreduced base spaces, we have the identification $T_{o} S=H^{1}\left(X, \Sigma_{L}\right)$.

For a polarized Hodge manifold $(X, L)$ denote by Aut $(X, L)$ the group of automorphisms of $(X, L)$; Aut $(X, L)=\left\{\varphi \in\right.$ Aut $\left.X ; \varphi^{*} L \cong L\right\}$, and by $\operatorname{Aut}_{0}(X, L)$ its identity component. It is known that $\operatorname{Aut}_{o}(X, L)$ is exactly the linear part $L(X)$ of $\operatorname{Aut}_{o} X$ in this case (cf. e.g. $[\mathbb{K} ; 9.4]$ ). Hence we get: 
Lermmma 5.7. Let $(X, L)$ be as above. Then $X$ is in $\mathscr{A}$, if and only if $\mathbb{A u t}_{o}(X, L)$ reduces to the identity.

In particular, if $X$ is in $\mathscr{A}$, in a $\mathbb{K}$ uranishi family $(f: \mathscr{X} \rightarrow S, \mathscr{L})$ of $(\mathbb{X}, \mathbb{L})$, $\operatorname{dim}$ Aut $\left(X_{s}, L_{s}\right)$ is independent of $s$. Then, similarly to Proposition 5.3 we get:

Proposition 5.\&. If $X$ is in $\mathscr{A}$, a Kuranishi family of $(X, L)$ is universal and gives also universal deformations of all nearby fibers.

In this case $G(X, L):=$ Aut $(X, L) / A_{u t}(X, L)=$ Aut $(X, L)$ is a finite subgroup of $G(X, \gamma), \gamma=c_{1}(L)$. It acts naturally on the $\mathbb{K}$ uranishi space $S(\mathbb{X}, L)$ as in the $\mathbb{K a ̈ h l e r}$ case.

To consider the global moduli space we fix a pair $(M, \alpha)$ as in the $\mathbb{K a ̈ h l e r}$ case with $\alpha \in \operatorname{Im}\left(H^{2}(M, \mathbb{Z}) \rightarrow H^{2}(M, \mathbb{R})\right.$ ) (cf. Remark 3.3). We denote by $\mathfrak{M A}_{H}$ the set of isomorphism classes of polarized non-uniruled Hodge manifolds $(\mathbb{X}, \mathbb{L})$ such that $\left(\mathbb{X}, c_{1}(\mathbb{L})\right)$ is diffeomorphic to $(M, \alpha)$ in the previous sense.

Theorem 5.9. The set $\mathfrak{M}_{H}$ carries a natural structure of a Hausdorff complex space with at most countably many connected components. Moreover, it carries a natural complex $\mathbb{V}$-structure.

$\mathfrak{M}_{H}$ with the complex structure in the above theorem is called the moduli space of polarized non-uniruled Hodge manifolds.

Remark 5.3. If one fixes a Hilbert polynomial $P$ for $(X, L)$ and considers the corresponding moduli space $\mathfrak{M P}_{H}^{P}$ as a subset of $\mathfrak{M}_{H}$, then it is open and closed in $\mathfrak{D U}_{H}$ and carries a natural structure of an algebraic space of finite type over $\mathbb{C}$; in fact $\mathfrak{D P}_{H}^{P}$ can be realized as a quotient of a suitable Hilbert scheme by a projective general linear group (cf. Sect. 11).

We give a sketch of a proof of Theorem 5.9 along the same line as in the $\mathbb{K}$ ähler case. We need an analogue of (5.4). Let $\underline{X}_{a}=(\mathscr{X} \rightarrow \mathbb{S}, \mathscr{L})$ and $\underline{X}_{a}^{\prime}=\left(\mathscr{X}^{\prime} \rightarrow \mathbb{S}, \mathscr{L}^{\prime}\right)$ be two polarized families of Hodge manifolds. Since we have the notions of isomorphisms of such families we have the natural functor $\underline{I s o m}_{S}\left(\mathscr{X}_{a}, \mathscr{X}_{a}^{\prime}\right):(\mathbb{A n} / S) \rightarrow(\mathbb{S e t s})$ analogous to the $\mathbb{K a ̈ h l e r}$ case. Denote by $\mathscr{X}=(\mathscr{X} \rightarrow S, \tilde{\gamma})$ and $\underline{X}^{\prime}=\left(\mathscr{X}^{\prime} \rightarrow \mathbb{S}, \tilde{\gamma}^{\prime}\right)$ the induced family of polarized $\mathbb{K a ̈ h l e r ~ m a n i f o l d s . ~}$

Proposition 5.10. The functor $\underline{I s o m}_{S}\left(\mathscr{X}_{a}, \mathscr{X}_{a}^{\prime}\right)$ is representable by a closed complex subspace $\underline{I s o m}_{S}\left(\mathscr{X}_{a}, \mathscr{X}_{a}^{\prime}\right)$ of $\underline{I}_{\operatorname{som}_{S}}\left(\mathscr{X}, \underline{\mathscr{X}}^{\prime}\right)$. Moreover, it is finite, i.e., proper with finite fibers, over $S$ if the fibers of $\mathscr{X}$ and $\mathscr{X}^{\prime}$ are not uniruled.

Proof. Let $\Phi: \underline{X} \times_{S} I \rightarrow \underline{X}^{\prime} \times_{S} I$ be the universal isomorphism of the induced polarized families of $\mathbb{K a ̈ h l e r}$ manifolds over $I:=\mathbb{I s o m}_{S}\left(\mathscr{X}, \mathscr{X}^{\prime}\right) \rightarrow \mathbb{S}$. Denote by $\mathscr{L}_{I}$ and $\mathscr{L}_{I}^{\prime}$ be the pull-backs of $\mathscr{L}$ and $\mathscr{L}^{\prime}$ to $\mathscr{X} \times_{S} I$ and $\mathscr{X}^{\prime} \times_{s} I$ respectively. Then $\left(\Phi^{*} \mathscr{L}_{I}^{\prime}\right) \otimes \mathscr{L}_{I}^{-1}$ defines a section of the relative $\operatorname{Picard}$ variety $\mathbb{P i c}\left(\mathscr{X} \times_{S} I / I\right) \rightarrow \mathbb{I}$. Its zero set $I_{0}:=\mathbb{I s o m}_{S}\left(\mathscr{X}_{a}, \mathscr{X}_{a}^{\prime}\right) \subseteq \mathbb{I}$ provided with the restriction of $\Phi$ to an isomorphism $\mathscr{X} \times_{S} I_{0} \neg \mathscr{X}^{\prime} \times_{s} I_{0}$ clearly represents $\underline{I s o m}_{S}\left(\mathscr{X}_{a}, \mathscr{X}_{a}^{\prime}\right)$. The last assertion follows from (5.4) and the fact that each fiber $\mathbb{I}_{0, s}$ is either empty or is a principal homogeneous space of Aut $\left(X_{s}, \mathbb{L}_{s}\right)$, which is finite. 
The rest of the proof of Theorem 5.9 is now formally just the same as in the case $\mathfrak{M}$. We note that in particular for any polarized Hodge manifold $(X, L)$ we have a natural map $\pi: S(X, L) \rightarrow \mathfrak{M}_{H}$ which induces a bijection $S(X, L) / G(X, L) \neg U(X, L):=$ $\pi(S(X, L))$, giving the local analytic structure of $\mathfrak{M}_{H}$; further, the collection $\{(S(X, L) \rightarrow$ $U(X, L) ; G(X, L))\}$ determines a complex $V$-structure of $\mathfrak{M}_{H}$ in the previous notations.

We now compare the two moduli spaces considered so far. First, let $(X, L)$ be a polarized Hodge manifold in general with the associated polarized Kähler manifold $(X, \gamma)$. Then a Kuranishi family $(f: \mathscr{X} \rightarrow S, \mathscr{L})$ of $(X, L)$ gives rise to the associated deformation of $(X, \gamma)$, which in turn induces a versal map

$$
r: S(X, L) \rightarrow S(X, \gamma)
$$

between the corresponding Kuranishi spaces. Under the identifications of the Zariski tangent spaces as in Remarks 5.1 and 5.2 the differential $r_{*}$ of $r$ at $o$ is induced by the natural homomorphism

$$
u: H^{1}(X, \Sigma) \rightarrow H^{1}\left(X, \Theta_{X}\right)_{\gamma}
$$

in the exact sequence (2.14).

We shall give a description of this morphism in terms of the relative Picard variety associated to the Kuranishi family $(f: \mathscr{X} \rightarrow S, \tilde{\gamma})$ of $(X, \gamma)$. Since the construction is more or less standard, we omit some technical details for which we refer to [Fu4]. Let $\Gamma:=\operatorname{Im}\left(R^{2} f_{*} \mathbb{Z} \rightarrow R^{2} f_{*} \mathbb{R}\right)$, considered also as a local system on $S$. Then, corresponding to the natural sheaf homomorphism $R^{1} f_{*} \mathcal{O}_{\mathscr{X}}^{*} \rightarrow \Gamma$ we get a homomorphism $\tau$ : Pic $\mathscr{X} / S \rightarrow \Gamma$ over $S$ of the relative Picard variety to $\Gamma$. Considering $\tilde{\gamma}$ as a section $S \rightarrow \Gamma$, we set $\operatorname{Pic}_{\gamma} \mathscr{X} / S:=\tau^{-1}(\tilde{\gamma}(S))$. Then $\operatorname{Pic}_{\gamma} \mathscr{X} / S$ is smooth over $S$. Since Aut $\mathscr{X} / S$ is also smooth by Proposition 5.2, with respect to the natural action of Aut $_{o} \mathscr{X} / S \rightarrow S$ on $\operatorname{Pic}_{\gamma} \mathscr{X} / S$ over $S$ we can construct the relative quotient of $\operatorname{Pic}_{\gamma} \mathscr{X} / S$ over $S$ :

$$
u: P_{\gamma}(\mathscr{X} / S) \rightarrow S \text {. }
$$

Furthermore, both the quotient map $q: \operatorname{Pic}_{\gamma} \mathscr{X} / S \rightarrow P_{\gamma}(\mathscr{X} / S)$ and the structure morphism $P_{\gamma}(\mathscr{X} / S) \rightarrow S$ turn out to be smooth.

The fiber over $o$ of $u$ is naturally identified with the quotient abelian variety $P_{\gamma}(X):=\operatorname{Pic}_{\gamma} X /$ Aut $_{o} X$, where $\operatorname{Pic}_{\gamma} X=\left\{L \in \mathbb{P i c}_{\gamma} X ; c_{1}(L)=\gamma\right\}$. Let $l$ and $\bar{l}$ be the points of $\operatorname{Pic}_{\gamma} X\left(\subseteq \operatorname{Pic}_{\gamma} \mathscr{X} / S\right)$ and $P_{\gamma}(X)\left(\subseteq P_{\gamma}(\mathscr{X} / S)\right)$ determined by $L$. We fix a Poincaré line bundle $\mathscr{L}_{\gamma}$ on $\mathscr{X} \times{ }_{s} \mathbb{P i c}_{\gamma} \mathscr{X} / S$, associated to some section $S \rightarrow \mathscr{X}$ of $f$. It is then formal to prove the following:

Proposition 5.11. Set $\mathbb{P}=P_{\gamma}(\mathscr{X} / S)$. Let $\overline{\mathscr{L}}_{\gamma}$ be the pull-back of $\mathscr{L}_{\gamma}$ to $\mathscr{X} \times{ }_{s} P$ via holomorphic section $\sigma:(P, \bar{l}) \rightarrow\left(\operatorname{Pic}_{\gamma} \mathscr{X} / S, l\right)$ to $q$. Then the family $\left(f_{P}: \mathscr{X} \times{ }_{S} P \rightarrow P, \overline{\mathscr{L}}_{\gamma}\right)$ is a Kuranishi family of $(X, L)$ with respect to any given isomorphism of $(X, L)$ with $\left(X_{u(\bar{l})}, \bar{L}_{\bar{l}}\right)$. In particular, $r: S(X, L) \rightarrow S(X, \gamma)$ is smooth with its fiber dimension $\operatorname{dim} H^{1}\left(X, \mathcal{O}_{\mathscr{X}}\right)-\operatorname{dim} H^{0}\left(X, \Theta_{X}\right)$.

Moreover, the natural action of $G(X, \gamma)=\operatorname{Aut}(X, \gamma) /$ Aut $_{o} X$ on $S$ lifts naturally to an action on $P$ and we get the induced proper surjective morphism

$$
\bar{q}: P_{\gamma}(\mathscr{X} / S) / G(X, \gamma) \rightarrow S(X, \gamma) / G(X, \gamma)
$$


At $\bar{l} \in P_{\gamma}(\mathscr{X} / S)$ this also is isomorphic to the morphism $\bar{r}: S(X, L) / G(X, L) \rightarrow S(X, \gamma) /$ $G(X, \gamma)$ induced by $r$ and the inclusion $G(X, L) \rightarrow G(X, \gamma)$.

By the definitions of the moduli spaces $\mathfrak{M}_{H}$ and $\mathfrak{M}$ we have a canonical map $v: \mathfrak{M}_{H} \rightarrow \mathfrak{M}$ which assigns to the isomorphism class of a polarized Hodge manifold $(X, L)$ the class of the induced polarized $\mathbb{K a ̈ h l e r}$ manifold $(X, \gamma)$. The induced map $v^{-1}(U(X, \gamma)) \rightarrow U(X, \gamma)$ is naturally identified with the morphism $\bar{q}$ above. Together with Proposition 4.10 this shows the following:

Theorem 5.112. The canonical map $v: \mathfrak{M}_{H} \rightarrow \mathfrak{M}$ above is a proper $\mathbb{V}$-smooth morphism of complex spaces with complex $\mathbb{V}$-structures.

In particular, if a point $m$ of $\mathfrak{M}$ is represented by a polarized $\mathbb{K} a ̈ h l e r$ manifold $(X, \gamma)$, then the fiber over $m$ is naturally identified with $\operatorname{Pic}_{\gamma} X / A u t ~(X, \gamma)$.

\section{§6. Extremal Kähler Mamifolds-Their Deformations and Moduli Spaces}

In this section we first review the definition and the basic properties of extremal Kähler manifolds, and then, construct the local and global moduli spaces of extremal Kähler and Hodge manifolds in the class $\mathscr{A}$ introduced in the previous section. The main point here is to show that for any metrically polarized family of $\mathbb{K a ̈ h l e r}$ manifolds in $\mathscr{A}$ an extremal Kähler metric given on a fiber extends uniquely and smoothly to all nearby fibers as a family of extremal $\mathbb{K a ̈ h l e r}$ metrics. This also forms the basis of the relations between these moduli spaces and those of the corresponding polarized manifolds defined in the previous section.

Let $(X, \gamma)$ be a polarized compact Kähler manifold. Let $U$ be the space of all the $C^{\infty}$ Kähler forms $\omega$ on $X$ which represent the $\mathbb{K a ̈ h l e r ~ c l a s s ~} \gamma \in H^{2}(X, \mathbb{R})$. According to Calabi [C1] [C2] we shall introduce the notion of extremal $\mathbb{K a ̈ h l e r}$ metrics as follows. First, we consider the functional $\Phi$ on $U$ defined by:

$$
\Phi(\omega):=\int_{X} R^{2} \omega^{n}, \quad \omega \in U, \quad n=\operatorname{dim} X .
$$

where $R=R(\omega)$ is the scalar curvature of the associated $\mathbb{K a ̈ h l e r}$ metric $g_{\omega}$. Then the Euler-Lagrange equation of $\Phi$ is given by the following (cf. [C1]):

$$
\bar{\partial}_{B} R=i \bar{\partial} \uparrow_{g} \bar{\partial} R=0, \quad \text { or } \quad \mathbb{R}_{; \bar{\beta}} \bar{\delta}=0 .
$$

Definition 6.1. Let $\omega$ be a $\mathbb{K a ̈ h l e r}$ form on $X$. Then $\omega$, or the associated $\mathbb{K}$ ähler metric $g_{\omega}$, is called extremal if it gives a critical point of the functional $\Phi$. If $\omega$ is extremal, we call the pair $(X, \omega)$ an extremal (compact) $\mathbb{K}$ ähler manifold.

From the definition it is clear that a metric with constant scalar curvature is extremal. Conversely, by (5.1) and (6.2) we obtain:

Proposition 6.1. If $X$ is in $\mathscr{A}$, then a Kähler form $\omega$ is extremal if and only if it is of constant scalar curvature.

Remark 6.1. Any $\mathbb{K}$ ähler-Einstein metric is of constant scalar curvature, and hence is extremal. Precisely, it occurs in one of the following three cases: 1) $c_{1}(\mathbb{X})<0$, and 
$\left.\gamma=-e c_{1}(X), 2\right) c_{1}(X)=0$ and $\gamma$ is arbitrary, and 3) $c_{1}(X)>0$, and $\gamma=e c_{1}(X)$, where $e$ is any positive real number. Indeed, a Kähler-Einstein metric is exactly a Kähler metric with constant scalar curvature in $\gamma$ in one of the above three cases. In the cases 1) and 2) $X$ is always in $\mathscr{A}$ (cf. e.g. [Fu1; Prop. 6.5]), and hence any extremal metric in $\gamma$ is Kähler-Einstein.

For any compact Kähler manifold $(X, \omega)$ we set

$$
\operatorname{Aut}(X, \omega)=\left\{h \in \text { Aut } X ; h^{*} \omega=\omega\right\} .
$$

This coincides with the group of isometries of $(X, \omega)$. Denote by $\operatorname{Aut}_{o}(X, \omega)$ its identity component. There are some necessary conditions on the structure of $\operatorname{Aut}_{o}(X, \omega) \subseteq$ Aut $_{o} X$ for $X$ to admit an extremal metric due to Matsushima, Lichnerowicz, and Calabi (cf. $[\mathrm{K}][\mathrm{C} 2])$ :

(6.3) Suppose that $\omega$ is extremal. Then $\operatorname{Aut}_{o}(X, \omega)$ is a maximal compact subgroup of Aut $_{o} X$. If, further, it is of constant scalar curvature, $L(X)$ is a complexification of $\operatorname{Aut}_{o}(X, \omega) L(X)$. In particular, if $X$ is in $\mathscr{A}$, $\operatorname{Aut}_{o}(X, \omega)=\operatorname{Aut}_{o} X$. If $X$ is not of constant scalar curvature, then $\operatorname{dim}\left(\operatorname{Aut}_{o}(X, \omega) \cap L(X)\right)>0$. Here $L(X)$ is the linear part of Aut $_{o} X$ (cf. Sect. 5).

The last condition gives a necessary condition for the existence of extremal Kähler metrics which gives rise to many examples of compact Kähler manifolds (not in $\mathscr{A}$ ) with no extremal metrics (Levine). On the other hand, Burns and de Bartolomeis $[B-B]$ recently have found examples of ruled surfaces in $\mathscr{A}$ and Kähler classes on them in which no extremal metrics exist (cf. also [Fu6]). For non-uniruled manifolds such examples seem to be unknown.

By Calabi [C2] it is also known that $L(X)$ acts transitively on each connected component of the set of extremal Kähler metrics; in particular if $X$ is in $\mathscr{A}$, this set is descrete (cf. Theorem 6.3 below).

Now we consider deformations of extremal manifolds.

Definition 6.2. A metrically polarized family $(f: \mathscr{X} \rightarrow S, \tilde{\omega})$ is said to be a (polarized) family of extremal Kähler manifolds if $\omega_{s}$ is extremal on $X_{s}$ for any $s$. An isomorphism of two such families $(f: \mathscr{X} \rightarrow S, \tilde{\omega})$ and $\left(f^{\prime}: \mathscr{X}^{\prime} \rightarrow S, \tilde{\omega}^{\prime}\right)$ is an isomorphism $\psi: \mathscr{X} \rightarrow \mathscr{X}^{\prime}$ of complex spaces over $S$ with $\psi^{*} \tilde{\omega}^{\prime}=\tilde{\omega}$ in the obvious sense.

Starting from these notions we get as in Definition 5.1 the notions of deformations of an extremal Kähler manifolds, universal deformations of such, etc.

Theorem 6.2. Let $(X, \omega)$ be an extremal compact Kähler manifold with $X$ in $\mathscr{A}$. Then $(X, \omega)$ posses a universal deformation $(f: \mathscr{X} \rightarrow S, \tilde{\omega})$ which induces also universal deformations of all nearby fibers. Furthermore, the associated deformation of the associated polarized Kähler manifold $(X, \gamma)$ is a Kuranishi family of $(X, \gamma)$.

We shall call a universal deformation of the theorem a Kuranishi family of $(X, \omega)$ and its base space $S=S(X, \omega)$ a Kuranishi space of $(X, \omega)$. The proof of the above theorem follows formally from the universality of a Kuranishi family of $(X, \gamma)$ and the following extension theorem of an extremal metric. (See also [Fu5].) 
Theorem 6.3. Let $(f: \mathscr{X} \rightarrow S, \tilde{\gamma})$ be a polarized family of compact $\mathbb{K}$ ähler manifolds and $o \in S$ a point. Suppose that $X_{o}$ is in $\mathscr{A}$ and $\gamma_{o}$ is represented by an extremal $\mathbb{K} a ̈ h l e r$ form $\omega_{o}$ on $X_{o}$. Then over a sufficiently small neighborhood $U$ of $o, \omega_{o}$ extends uniquely to a $C^{\infty}$ family $\tilde{\omega}=\left\{\omega_{s}\right\}_{s \in U}$ of extremal Kähler forms $\omega_{s}$ on $X_{s}$ which represents $\gamma_{s}$ for all $s \in U$.

Proof. The proof depends on the fact that the equation " $R=$ const" leads to a (non-linear) elliptic equation of order four, and uses the implicit function theorem.

Now fix any metric polarization $\tilde{\omega}^{\prime}$ for $(f, \tilde{\gamma})$ with $\omega_{o}^{\prime}=\omega_{o}$ by Lemma 3.2. Any other metric polarization is then of the form $\omega^{\prime}+i \partial_{\mathscr{X} / S} \bar{\partial}_{\mathscr{X} / S} \varphi$ for some real $C^{\infty}$ function $\varphi$ on $\mathscr{X}$ locally over $S$ (cf. Lemma 3.5 and (3.2)). Thus our $\omega_{s}$ should be of the form $\omega_{s}^{\prime}+i \partial_{s} \bar{\partial}_{s} \varphi_{s}$ for some $C^{\infty}$ function $\varphi_{s}$ on $X_{s}$, which is uniquely determined if we assume $\int_{X_{s}} \varphi_{s} \omega_{s}^{\prime n}=0$, where $n$ is the dimension of the fibers of $f$.

In order to treat the case of a singular base space rigourously, we first remark the following: The problem is local with respect to $S$. So we can assume that $S$ is a closed analytic subset of a domain $D$ in $\mathbb{C}^{m}$, and there exist a $C^{\infty}$ trivialization $\tau: \mathscr{X} \rightarrow X \times S$ over $S$ and a $C^{\infty}$ family $\left\{J_{s}\right\}_{s \in D}$ of almost complex structures on $X$ which induce for $s \in \mathbb{S}$ the complex structures of $X_{s}$ via $\tau_{s}:=\tau \mid X_{s}$, where $X=X_{o}$ (cf. $[\mathbb{K u}]$ ). Thus $\partial$ - and $\bar{\partial}$-operators on the fibers $X_{s}$ come from a $C^{\infty}$ family of differential operators $\partial_{s}$ and $\bar{\partial}_{s}, s \in D$.

Take and fix a positive integer $k \geqq n+5$. Let $H^{k}(X)$ be the Sobolev space of real functions on $X$, whose derivatives up to order $k$ exist in the distributional sense and are square integrable. By Sobolev's lemma every element of $H^{k}(X)$ is at least of class $C^{4}$. We set

$$
W=\left\{(s, \psi) \in S \times H^{k}(X) ; \omega_{s}^{\prime}+i \partial_{s} \bar{\partial}_{s}\left(\psi \cdot \tau_{s}\right) \text { is } \mathbb{K a ̈ h l e r ~ o n ~} X_{s}\right\} .
$$

Set $\omega=\omega_{o}$ and denote by $H_{o}^{k}(X)$ the closed subspace of $H^{k}(X)$ defined by $\int_{X} \psi \omega^{n}=0$. Then the map

$$
\begin{aligned}
& \mathbb{F}:\left(\mathbb{S} \times H_{o}^{k}(X)\right) \cap W \rightarrow H_{o}^{k-4}(X), \\
& F(s, \psi)=\mathbb{R}(s, \psi)-\left(\int_{X} \mathbb{R}(s, \psi) \omega^{n} / \int_{X} \omega^{n}\right),
\end{aligned}
$$

where $R(s, \psi)$ is the scalar curvature of $\omega_{s}^{\prime}+i \partial_{s} \bar{\partial}_{s}\left(\psi \tau_{s}\right)$ pulled-back to $X$ by $\tau_{s}$. One can see that $F$ is of class $C^{\infty}$ (also for singular $S$ ). We first look for a solution of $F(s, \phi(s))=0$, where $\phi: S \rightarrow H_{o}^{k}(X)$ is a $\mathbb{C}^{\infty}$ map.

Now the second partial derivative $D_{2} F$ of $F$ at $(o, 0)$ is the linear map $D: H_{o}^{k}(X) \rightarrow$ $H_{o}^{k-4}(X)$ which assigns to a function $\psi \in H_{o}^{k}(X)$ the function $-\bar{\partial}_{B}^{*} \bar{\partial}_{B} \psi$ in the notations of Sect. 2. (See (2.5) and Lemma 2.1 of [C2]. The contribution from the second term of (6.4) clearly yields zero.) Thus $\left(D_{2} F\right)(\psi)=0$ implies $\bar{\partial}_{B} \psi=0$. By (5.1) and our assumption that $X_{s} \in \mathscr{A}$ we get that $\psi$ is constant, which means by our normalization $\psi=0$.

Since $D$ is a strongly elliptic operator, this implies that $D$ possesses a bounded inverse. Hence, by the implicit function theorem, over a neighborhood of $o$ there exists a unique $C^{\infty} \operatorname{map} \phi: S \rightarrow H_{o}^{k}(X)$ with $\phi(o)=0$ and $F(s, \phi(s))=0$. It remains to show 
that $\phi(s)$ is of class $C^{\infty}$ for all $s$. Since $\phi(s)$ is at least of class $C^{4}$ as we have remarked above, this in fact follows from the next lemma, (thus completing the proof).

Lemmmæ 6.4. Let $(X, \omega)$ be a Kähler manifold with a $C^{\infty}$ Kähler form $\omega$. Let $\varphi$ be a real function of class $C^{4}$ on $X$ such that $\omega+i \partial \bar{\partial} \varphi$ is again a Kähler form and its scalar curvature is constant. Then $\varphi$ is a $C^{\infty}$ function.

Proof. We use the regularity theorem of Agmon-Douglis-Nirenberg [A-D-N; Th. 12.1] (cf. also [Bes]). For any real function $\psi$ on $X$ such that $\omega(\psi)$ is a Kähler form, denote by $R(\psi)$ the scalar curvature of $\omega(\psi)$. Then the condition " $R(\psi)=$ const." leads to a nonlinear differential equation $P(\psi)=0$ of order 4 in $\psi$ with $C^{\infty}$ coefficients, and $\varphi$ is a $C^{4}$ solution of this equation. The first variation of this equation at $\varphi$ is exactly the elliptic equation $\bar{\partial}_{B}^{*} \bar{\partial}_{B} \psi=0$ as we have noted in the previous proof, where $\bar{\partial}_{B}$ and $\bar{\partial}_{B}^{*}$ are now taken with respect to the $\mathbb{K}$ ähler form $\omega(\varphi)$. Therefore, by [A-D-N] we get that $\varphi$ is of class $C^{\infty}$.

Remark 6.2. The above lemma and Theorem 6.3 are also true even if we replace " $C^{\infty}$ " by "real analytic" there.

In order to pass from Theorem 6.2 to global moduli space we need a result analogous to (5.4). In this case, however, we can stay in the class $\mathscr{A}$ which is larger than the class of non-uniruled manifolds. Let $\underline{X}_{e}:=(\mathscr{X} \rightarrow S, \tilde{\omega})$ and $\underline{X}_{\bar{e}}\left(\mathscr{X}^{\prime} \rightarrow S, \tilde{\omega}^{\prime}\right)$ be two families of extremal compact Kähler manifolds. Since we have the notions of isomorphisms of such families we have the natural functor

$$
\underline{\operatorname{Isom}}_{S}\left(\underline{\mathscr{X}}_{e}, \underline{X}_{e}^{\prime}\right):(\mathrm{An} / S) \rightarrow(\text { Sets }) .
$$

Denote by $\mathscr{X}$ and $\underline{X}^{\prime}$ the underlying polarized families of Kähler manifolds so that we have the natural morphism of functors $\underline{I s o m}_{S}\left(\underline{\mathscr{X}}_{e}, \underline{X}_{e}^{\prime}\right) \rightarrow \underline{I s o m}_{S}\left(\underline{\mathscr{X}}, \underline{\mathscr{X}}^{\prime}\right)$.

Proposition 6.5. Suppose that $X_{s}$ and $X_{s}^{\prime}$ are in $\mathscr{A}$ for all s. Then $\underline{I s o m}_{S}\left(\underline{X}_{e}, \underline{X}_{e}^{\prime}\right)$ is representable by an open and closed subspace $\underline{I s o m}_{S}\left(\underline{X}_{e}, \underline{X}_{e}^{\prime}\right)$ of $\underline{I s o m}_{S}\left(\mathscr{X}, \underline{X}^{\prime}\right)$ which is proper over $S$.

Proof. The fact that $\underline{I s o m}_{S}\left(\mathscr{X}_{e}, \underline{X}_{e}^{\prime}\right)$ is representable by an open subset of $\operatorname{Isom}_{S}\left(\mathscr{X}, \underline{X}^{\prime}\right)$ follows easily from Theorem 6.3. In fact, let $\Phi: \underline{X} \times_{S} I \rightarrow \underline{X}^{\prime} \times_{S} I$ be the universal isomorphism, where $I=\operatorname{Isom}_{S}\left(\mathscr{X}, \underline{X}^{\prime}\right)$. Then the zero of $\Phi^{*} \tilde{\omega}_{I}^{\prime}-\tilde{\omega}_{I}$, considered on $I$, is an open and closed subset of $I$ by Theorem 6.3. This serves as $\operatorname{Isom}_{S}\left(\mathscr{X}_{e}, \underline{X}_{e}^{\prime}\right)$. It remains to show that this set is proper over $S$. Take any sequence of points $S_{v}$ of $S$ converging to a point $o \in S$, and a sequence of isomorphisms $\varphi_{v}:\left(X_{s_{v}}, \omega_{s_{v}}\right) \rightarrow$ $\left(X_{s_{v}}^{\prime}, \omega_{s_{v}}^{\prime}\right)$. Since these are isometries with respect to the underlying Riemannian metrics, by Lemma 6.8 of [Fu-Sch] for any $m>0$ a sequence of $\left\{\varphi_{v}\right\}$ converges in the $C^{m}$ topology to a $C^{m}$ isometry $\varphi: X_{o} \rightarrow X_{o}^{\prime}$ with respect to the underlying Riemannian metrics. However, as a $C^{m}$-limit of biholomorphic maps $\varphi_{v}, \varphi$ also is biholomorphic. Hence the convergence actually takes place in $\operatorname{Isom}_{S}\left(\underline{x}_{e}, \underline{X}_{e}^{\prime}\right)$. So the latter is proper over $S$.

Now let $(X, \omega)$ be an extremal compact Kähler manifold, and $(f: \mathscr{X} \rightarrow S, \tilde{\omega})$ a Kuranishi family of $(X, \omega)$. By the universality of $(f, \tilde{\omega})$ combined with $(6.3)$ and Proposition 5.2, we have a natural action of the finite group $G(X, \omega):=\operatorname{Aut}(X, \omega) /$ 
Aut $_{o} X$ on $S(X, \omega)$. Moreover, from Proposition 6.5 we conclude that for any points $r$ and $s$ of $S(X, \omega),\left(X_{r}, \omega_{r}\right)$ and $\left(X_{s}, \omega_{s}\right)$ are isomorphic if and only if $r=k(s)$ for some $k \in G(X, \omega)$.

It is then standard to construct a global moduli space of extremal $\mathbb{K a ̈ h l e r ~ m a n i f o l d s ~}$ as in the case of moduli space of compact $\mathbb{K}$ ähler manifolds. We first fix a compact $\mathbb{C}^{\infty}$ manifold $M$ and a class $\alpha \in H^{2}(M, \mathbb{R})$. Denote by $\mathfrak{M}_{e}$ the set of isomorphism classes of compact extremal Kähler manifolds $(X, \omega)$ with $X$ in $\mathscr{A}$ such that $(X, \gamma), \gamma=[\omega]$, is diffeomorphic to $(M, \alpha)$.

Theorem 6.6. The set $\mathfrak{M}_{e}$ carries a natural structure of a Hausdorff reduced complex space with a natural complex $\mathbb{V}$-structure.

We call $\mathfrak{M}_{e}$ with the above complex structure the moduli space of extremal compact Kähler manifolds (in the class $\mathscr{A}$ ). The complex $V$-structure, and hence also the local analytic structure, of $\mathfrak{M}_{e}$ is described as follows. If $m$ is a point of $\mathfrak{M}_{e}$ represented by an extremal Kähler manifold $(X, \omega)$, the image $U(X, \omega)$ of the natural map $S(X, \omega) \rightarrow$ $\mathfrak{M}_{e}$ forms an open neighborhood of $m$ and it is identified with $S(X, \omega) / G(X, \omega)$. Then the collection $\{S(X, \omega) \rightarrow U(X, \omega) ; G(X, \omega)\}$ with $S(X, \omega)$ sufficiently small as in Sect. 5 give the complex $V$-structure of $\mathfrak{M}_{e}$.

Let $\mathfrak{M}^{\prime}$ be the subset of $\mathfrak{M}_{e}$ consisting of the classes non-uniruled manifolds. Then we have a natural map $\kappa: \mathfrak{M}_{e}^{\prime} \rightarrow \mathfrak{M}$ of $\mathfrak{M}^{\prime}$ into the moduli space $\mathfrak{M}$ of polarized Kähler manifolds considered in Sect. 5.

Theorem 6.7. $\mathfrak{M}_{e}^{\prime}$ is an open and closed subspace $\mathfrak{M}_{e}$. The natural morphism $k: \mathfrak{M}_{e}^{\prime} \rightarrow \mathfrak{M}$ is an open holomorphic map of complex spaces with discrete fibers, which correspond bijectively to the set of extremal Kähler metrics of the given polarized Kähler manifolds considered modulo the action of $\operatorname{Aut}(X, \gamma)$.

For the first assertion see (5.4). The second assertion follows from the following local description of the map $\kappa$ in terms of $V$-structures. Let $m \in \mathfrak{M}_{e}^{\prime} \subseteq \mathfrak{M}_{e}$ and $(X, \omega)$ be as before. Let $(X, \gamma)$ be the associated polarized $\mathrm{Kähler}$ manifolds. By Theorem 6.2 we may identify $S=S(X, \omega)$ with $S(X, \gamma)$. The natural inclusion $\operatorname{Aut}(X, \omega) \leqq \operatorname{Aut}(X, \gamma)$ induces an inclusion $G(X, \omega) \subseteq G(X, \gamma)$, and then a finite morphism

$$
S(X, \omega) / G(X, \omega) \rightarrow S(X, \gamma) / G(X, \gamma) .
$$

This can be identified with the map $\kappa$ in a neighborhood of $m$ with respect to the natural identifications of $S / G(X, \omega)$ and $S / G(X, \gamma)$ with open neighborhoods of $m \in \mathfrak{M}_{e}^{\prime}$ and $\kappa(\mathfrak{m}) \in \mathfrak{M}$ respectively.

We shall study a variation of the previous result for Hodge manifolds.

Definition 6.3. An extremal Hodge manifold is a triple $(X, \omega, L)$, where $(X, \omega)$ is an extremal Kähler manifold and $(X, L)$ is a polarized Hodge manifold such that $\omega$ represents the first chern class $c_{1}(L)$. A (polarized) family of extremal Hodge manifolds is a metrically polarized family $(f: \mathscr{X} \rightarrow S, \omega, \mathscr{L})$ of Hodge manifolds (cf. Def. 3.8) such that $(f, \tilde{\omega})$ is a family of extremal Kähler manifolds. An isomorphism of two such families $(f: \mathscr{X} \rightarrow S, \tilde{\omega}, \mathscr{L})$ and $\left(f^{\prime}: \mathscr{X}^{\prime} \rightarrow S, \tilde{\omega}^{\prime}, \mathscr{L}^{\prime}\right)$ is an isomorphism $\varphi: \mathscr{X} \underset{\rightarrow}{\rightarrow} \mathscr{X}^{\prime}$ of com- 


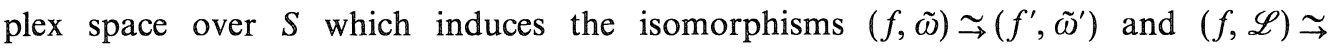
$\left(f^{\prime}, \mathscr{L}^{\prime}\right)$ of the underlying families (cf. Def. 6.2 and 3.7).

Theorem 6.8. Any extremal Hodge manifold $(X, \omega, \mathscr{L})$ with $X$ in $\mathscr{A}$ posesses a universal deformation $(f: \mathscr{X} \rightarrow S, \tilde{\omega}, \mathscr{L})$ which also induces universal deformations of all nearby fibers. Furthermore, the associated deformation $(f: \mathscr{X} \rightarrow S, \mathscr{L})$ of the polarized Hodge manifolds is a Kuranishi family of $(X, L)$.

Proof. Let $(f: \mathscr{X} \rightarrow S, \mathscr{L}),\left(X_{o}, L_{o}\right)=(X, L)$, be a $\mathbb{K}$ uranishi family of $(X, L)$. Then by Theorem 6.3 there exists a unique extremal metric polarization $\tilde{\omega}=\left\{\omega_{s}\right\}$ for $(f, \mathscr{L})$ with $\omega_{o}=\omega$. It is immediate to see that the resulting triple $(f, \tilde{\omega}, \mathscr{L})$ gives a desired universal family.

As before, we get a Kuranishi family $S=S(X, \omega, L)$ and the group $G(X, \omega, L):=$ $\operatorname{Aut}(X, \omega, L) /$ Aut $_{o} X$ acting on $S$. From the last assertions of Theorems 6.2 and 6.8 together with Proposition 5.11 we have:

Corollary 6.9. The natural morphism $S(X, \omega, L) \rightarrow S(X, \omega)$ is smooth.

As before, in order to pass from a local to global moduli space we need a result on the structure of isomorphisms of families. Let $(f: \mathscr{X} \rightarrow S, \tilde{\omega}, \mathscr{L})$ be a family of extremal Hodge manifolds. We set $\underline{x}_{e}=(f, \tilde{\omega}), \underline{X}_{a}=(f, \mathscr{L})$ and $\underline{X}_{a, e}=(f, \tilde{\omega}, \mathscr{L})$. If $\left(f^{\prime}: \mathscr{X}^{\prime} \rightarrow\right.$ $\left.S, \tilde{\omega}^{\prime}, \mathscr{L}^{\prime}\right)$ is another such family, $\mathscr{X}_{e}^{\prime}, \mathscr{X}_{a}^{\prime}$, and $\mathscr{X}_{a, e}^{\prime}$ are defined similarly. We get then a functor $\operatorname{Isom}_{S}\left(\underline{X}_{a, e}, \underline{X}_{a, e}^{\prime}\right):(\mathrm{An} / S) \rightarrow($ Sets $)$ in the obvious way. Then the following is immediate from the definitions and Propositions 5.10 and 6.5.

Proposition 6.10. Suppose that $X_{s}$ and $X_{s}^{\prime}$ are in $\mathscr{A}$ for all $s$. Then the functor $\operatorname{Isom}_{S}\left(\underline{X}_{a, e}, \underline{X}_{a, e}^{\prime}\right)$ defined above is representable by the subspace $I_{a, e}:=\operatorname{Isom}_{S}\left(\underline{X}_{a}, \underline{X}_{a}^{\prime}\right) \cap$ $\operatorname{Isom}_{S}\left(\mathscr{X}_{e}, \underline{X}_{e}^{\prime}\right)\left(\subseteq \operatorname{Isom}_{S}\left(\mathscr{X}, \underline{X}^{\prime}\right)\right)$. In particular, the natural morphism $I_{a, e} \rightarrow S$ is finite.

Let $M$ be a compact $C^{\infty}$ manifold and $\alpha \in \operatorname{Im}\left(H^{2}(M, \mathbb{Z}) \rightarrow H^{2}(M, \mathbb{R})\right)$ be an integral element. Denote by $\mathfrak{M}_{H, e}$ the set of isomorphism classes in the obvious sense of extremal Hodge manifolds $(X, \omega, L)$ with $X$ in $\mathscr{A}$. From Proposition 6.10 we get as before:

Theorem 6.11. The set $\mathfrak{M}_{H, e}$ carries a natural structure of a Hausdorff reduced complex space with a natural complex V-structure.

Let $\mathfrak{M}_{H, e}^{\prime}$ be the subset of $\mathfrak{M}_{H, e}$ consisting of isomorphism classes of $(X, \omega, L)$ with $X$ non-uniruled. Then we have the following commutative diagram of natural maps

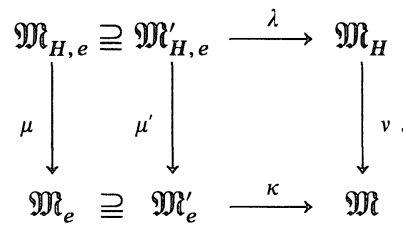

Then as Theorems 5.12 and 6.7 we get:

Theorem 6.12. $\mathfrak{M}_{H, e}^{\prime}$ is an open and closed subspace of $\mathfrak{M}_{H, e}$, and all the maps in (6.5) are morphisms of complex spaces, where $\lambda$ and $\kappa$ are open with discrete fibers, and $\mu$, 
$\mu^{\prime}$ and $v$ are proper surjective and $\mathbb{V}$-smooth. The fiber of $\mu$ over a point of $\mathfrak{M A}_{e}$ represented by an extremal $K$ ähler manifold $(X, \omega)$ is naturally identified with $\mathbb{P i c}_{\gamma} X /$ $\operatorname{Aut}(X, \omega)$, where $\gamma=[\omega]$.

The local description of $\mu$ similar to the one for $v$ in Theorem 5.12 is given by the following commutative diagram

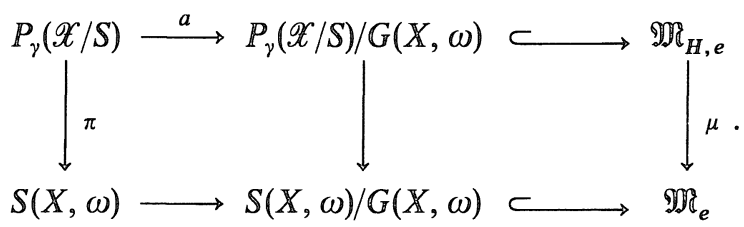

Here, as a map into $\mathfrak{M}_{H, e}, a$ is clearly a $V$-morphism in the sense of Definition 1.9; in fact, locally at each point of $P_{\gamma}(\mathscr{X} / S) \pi$ is locally isomorphic to the natural morphism $S(X, \omega, L) \rightarrow S(X, \omega)$.

\section{§7. Generalized Weil-Petersson Metrics and their Kähler Property}

In this section, first for any polarized (effective) family of extremal Kähler and Hodge manifolds we define the generalized Weil-Petersson metric, which is a hermitian metric on the base space of the family. This will be done here as a formal consequence of the results of Sections 2 and 5. We also see that this agrees with the classical definition in the Kähler-Einstein case. We then proceed to state our results concerning its Kähler property in a series of theorems. In the Hodge case our result gives more precisely the representation of the Weil-Petersson form as a certain fiber integral along the given family. The Kähler property is a direct consequence of this in the Hodge case, even for singular base spaces thanks to the recent result of Varouchas [V]. The proof of the integral formula, which we formulate more generally for any refined metrically polarized families of $\mathbb{K} a ̈ h l e r$ manifolds, will be given in Section 8. The $\mathbb{K}$ ähler property for families of $\mathbb{K a ̈ h l e r ~ m a n i f o l d s ~ w i l l ~ b e ~ d e d u c e d ~ f r o m ~ t h i s ~ i n ~ S e c t i o n ~ 9 . ~ T h e ~ g l o b a l ~}$ consequence of the above results on the moduli spaces $\mathfrak{M}_{H, e}$ and $\mathfrak{M}_{e}$ will also be given.

In the rest of the paper, "Weil-Petersson" will often be abbreviated to WP.

We now give the definitions of generalized WP metrics. For the definition however we may consider any polarized family (not necessarily extremal). We start with the Kähler case. Let $(f: \mathscr{X} \rightarrow S, \tilde{\omega})$ be a metrically polarized family of compact $\mathbb{K a ̈ h l e r}$ manifolds. For any $s \in S$, composing the Kodaira-Spencer map $\rho_{s}: T_{s} S \rightarrow H^{1}\left(X_{s}, \Theta_{X_{s}}\right)_{\gamma_{s}}$ associated to the underlying polarized family $(f, \tilde{\gamma})$ with the natural isomorphism $\check{\mu}_{s}^{-1}: H^{1}\left(X_{s}, \Theta_{X_{s}}\right)_{\gamma_{s}} \rightarrow \check{\mathscr{H}}_{s}^{1}$ (Theorem 2.5), we get a $\mathbb{C}$-linear map

$$
\check{\rho}_{s}: T_{s} S \rightarrow \check{\mathscr{H}}_{s}^{1}
$$

where $\check{\mathscr{H}}_{s}^{1}=\check{\mathscr{H}}_{B_{s}}^{1}$ is the essential harmonic space in $B_{s}^{1}:=B^{1}\left(X_{s}, \omega_{s}\right)$ (cf. Def. 2.1). Then by pulling back the hermitian inner product of the hermitian space $\breve{\mathscr{H}}_{s}^{1}$ by $\check{\rho}_{s}$ we get a natural (positive semi-definite) hermitian inner product $h_{s}$ on $T_{s} S$. If $(f, \tilde{\omega})$ is effective, i.e., so is $(f, \tilde{\gamma})$, then $h_{s}$ is positive definite. Denote also by $\omega_{s}$ the imaginary part of $h_{s}$, 
which is an alternating form on the (real underlying space of) the Zariski tangent space $T_{s} S$, considered also as an element of $\Lambda^{1,1} T_{s}^{*} S$.

Definition 7.1. Suppose that $(f, \tilde{\omega})$ is effective. Then the generalized Weil-Petersson metric on $S$ associated to $(f, \tilde{\omega})$ is the collection $h_{W P}:=\left\{h_{s}\right\}_{s \in S}$ of hermitian inner products $h_{s}$ on $T_{s} S$ defined above. The collection $\omega_{W P}:=\left\{\omega_{s}\right\}_{s \in S}$ is called the generalized Weil-Petersson form on $S$ associated to $(f, \tilde{\omega})$.

By the functoriality of $\check{\rho}_{s}$ it is immediate to see that if $\left(f^{\prime}: \mathscr{X}^{\prime} \rightarrow S^{\prime}, \tilde{\omega}^{\prime}\right)$ is another such family and $\psi$ is an isomorphism of two families $(f, \tilde{\omega})$ and $\left(f^{\prime}, \tilde{\omega}^{\prime}\right)$, then $\psi$ induces a biholomorphic isometry of hermitian spaces $\left(S, h_{W P}(S)\right)$ and $\left(S^{\prime}, h_{W P}\left(S^{\prime}\right)\right)$.

Next, we consider the case of a polarized family $(f: \mathscr{X} \rightarrow S, \tilde{\omega}, \mathscr{L})$ of Hodge manifolds. Composing the Kodaira-Spencer map $\hat{\rho}_{s}: T_{s} S \rightarrow H^{1}\left(X_{s}, \Sigma_{s}\right)$ associated to the underlying polarized family $(f, L)$ with the natural isomorphism $\mu_{s}^{-1}: H^{1}\left(X_{s}, \Sigma_{s}\right) \rightarrow \mathscr{H}_{s}^{1}$ (2.15) we get a $\mathbb{C}$-linear map

$$
\bar{\rho}_{s}: T_{s} S \rightarrow \mathscr{H}_{s}^{1},
$$

where $\Sigma_{s}=\Sigma_{L_{s}}$ and $\mathscr{H}_{s}^{1}=\mathscr{H}_{B_{s}}^{1}$. Then, by pulling back the hermitian inner product on the hermitian space $\mathscr{H}_{s}^{1}$ by $\bar{\rho}_{s}$ we get a natural hermitian (possibly semidefinite) inner product $\hat{h}_{s}$ on $T_{s} S$. If the family is effective, i.e., if so is $(f, \mathscr{L})$, then $\hat{h}_{s}$ is positive definite. As in the Kähler case denote by $\hat{\omega}_{s}$ the imaginary part of $\hat{h}_{s}$.

Definition 7.2. Suppose that $(f, \tilde{\omega}, \mathscr{L})$ is effective. Then the generalized $W P$ metric associated to $(f, \tilde{\omega}, \mathscr{L})$ is the collection $\hat{h}_{W P}=\left\{\hat{h}_{s}\right\}_{s \in S}$ of hermitian inner products on $T_{s} S$ defined above. The collection $\hat{\omega}_{W P}:=\left\{\hat{\omega}_{s}\right\}_{s \in S}$ is called the generalized WP form associated to $(f, \tilde{\omega}, \mathscr{L})$.

Similar assertion on the functoriality of $\hat{h}_{W P}$ is true as for the case of a family of Kähler manifolds.

It turns out to be useful to consider $\hat{h}_{W P}$ also for a general refined metrically polarized family $(f: \mathscr{X} \rightarrow S, \tilde{\omega}, \eta)$ of Kähler manifolds (cf. Def. 3.4). Let $\hat{\rho}_{s}: T_{s} S \rightarrow H^{1}\left(X_{s}\right.$, $\Sigma_{s}$ ) be the Kodaira-Spencer map associated to the pair $(f, \eta)$ (Def. 4.1). Then in the same manner as above, composing $\hat{\rho}_{s}$ with $\mu_{s}^{-1}$ we get a naturally induced hermitian inner product $\hat{h}_{s}$ on each $T_{s} S$, and $\hat{h}_{s}$ is positive definite if $(f, \tilde{\omega}, \eta)$ is effective, i.e., so is $(f, \eta)$. Similarly, $\hat{\omega}_{s}$ is defined for each $s$.

Definition 7.3. Let $(f, \tilde{\omega}, \eta)$ be an effective, refined metrically polarized family as above. Then the collections $\hat{h}_{W P}=\left\{\hat{h}_{s}\right\}_{s \in S}$ and $\hat{\omega}_{W P}=\left\{\hat{\omega}_{s}\right\}_{s \in S}$ are respectively called the generalized WP metric, and form, associated to $(f, \tilde{\omega}, \eta)$.

Remark 7.1. 1) If $(f, \tilde{\omega}, \eta)$ comes from a family $(f, \tilde{\omega}, \mathscr{L})$ of Hodge manifolds (cf. Remark 3.4), then the generalized WP metrics in Definitions 7.2 and 7.3 coincide as is clear from the definitions. 2) If the first Betti number $b_{1}\left(X_{s}\right)=0$ for any $s$, then $\mathscr{H}_{s}^{1}=\check{\mathscr{H}}_{s}^{1}$ and $\hat{\rho}_{s}=\check{\rho}_{s}$; therefore we always have $\hat{h}_{W P}=h_{W P}$ and $\hat{\omega}_{W P}=\omega_{W P}$. In this case $\eta$ is uniquely determined by $(f, \tilde{\omega})$ (cf. Cor. 3.7) and hence $\hat{h}_{W P}$ depends only on $(f, \tilde{\omega})$. 
We shall obtain a more manageable expression for $\hat{\omega}_{W P}$ by using admissible $(1,1)$-forms. So for $(f, \tilde{\omega}, \eta)$ as above, we fix an admissible $(1,1)$-form $\omega_{\mathscr{X}}$ which is mapped to $\eta$ (cf. Prop. 3.6) and let $\xi_{s}: \mathbb{T}_{s} S \rightarrow \mathbb{B}_{s}^{1}$ be the linear map associated to the horizontal distribution $\mathbb{T}^{H}\left(\omega_{\mathscr{X}}\right)$. Composing $\xi_{s}$ with the orthogonal projection $B_{s}^{1} \rightarrow \mathscr{H}_{s}^{1}$ we get a natural $\mathbb{C}$-linear map $\rho_{s}\left(\omega_{\mathscr{X}}\right): \mathbb{T}_{s} S \rightarrow \mathscr{H}_{s}^{1}$. Then we get as before a hermitian inner product $\hat{h}_{s}\left(\omega_{\mathscr{X}}\right)$ and an alternating form $\hat{\omega}_{s}\left(\omega_{\mathscr{X}}\right)$ on $\mathbb{T}_{s} S$. We set $\hat{\omega}_{W P}\left(\omega_{\mathscr{X}}\right)=\left\{\hat{\omega}_{s}\left(\omega_{\mathscr{X}}\right)\right\}$ and call it the generalized WP form associated to $\left(f, \omega_{\mathscr{X}}\right)$. Then by Lemmas 4.9 and 4.10 and the above definitions the following is clear.

Lemma 7.1. Let $(f: \mathscr{X} \rightarrow S, \tilde{\omega}, \eta)$ be an effective refined metrically polarized family. Let $\omega_{\mathscr{X}}$ be an admissible $(1,1)$-form for $(f, \tilde{\omega})$ with $\eta=\delta_{0}\left(\omega_{\mathscr{X}}\right)$. Then the generalized WP forms associated to $(f, \tilde{\omega}, \eta)$ and $\left(f, \omega_{\mathscr{X}}\right)$ coincide; $\hat{\omega}_{W P}=\hat{\omega}_{W P}\left(\omega_{\mathscr{X}}\right)$.

We now consider the special case of families of $\mathbb{K}$ ähler-Einstein manifolds. Let $(f: \mathscr{X} \rightarrow S, \tilde{\omega})$ be an effective family of $\mathbb{K}$ ähler-Einstein manifolds. First, if $\omega_{s} \in m c_{1}\left(X_{s}\right)$ for some nonzero integer $m,\left(f, \tilde{\omega}, \mathbb{K}_{\mathscr{X} / S}^{m}\right)$ is a family of extremal Hodge manifolds and we have the associated generalized WP metric $\hat{h}_{W P}$. Propositions 4.12, 2.8 and Lemma 7.1 yield:

Proposition 7.2. Let $(f: \mathscr{X} \rightarrow S, \tilde{\omega})$ be as above. The two generalized WP metrics $\hat{h}_{W P}$ and $h_{W P}$ on $S$ coincide.

Next, we compare the generalized WP metric with the original Weil-Petersson metric considered in [Koi], [Sch4], [Si]. In this case by Proposition 2.7 we have the natural isomorphisms

$$
H^{1}\left(X_{s}, \Theta_{X_{s}}\right)_{\gamma_{s}} \cong H^{1}\left(X_{s}, \Theta_{X_{s}}\right) \cong \mathscr{H}_{A_{s}}^{1} \quad \text { if } \quad \omega_{s}=r c_{1}\left(X_{s}\right), \quad r \neq 0
$$

and

$$
H^{1}\left(X_{s}, \Theta_{X_{s}}\right)_{\gamma_{s}} \cong \mathscr{H}^{1} \quad \text { if } \quad c_{1}\left(X_{s}\right)=0
$$

where $\mathscr{H}_{A_{s}}^{1}$ is the space of vector valued harmonic $(0,1)$-forms with respect to the given

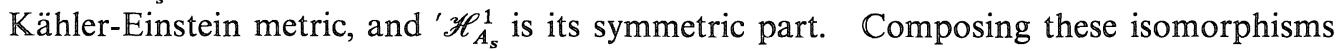
with $\rho_{s}: T_{s} S \rightarrow H^{1}\left(X_{s}, \Theta_{X_{s}}\right)_{\gamma_{s}}$ we obtain a natural $\mathbb{C}$-linear map $\rho_{s}^{0}: \mathbb{T}_{s} S \rightarrow \mathscr{H}_{A_{s}}^{1}$ (resp. ' $\mathscr{H}_{A_{s}}^{1}$ ). This induces as before a natural hermitian inner product on $\mathbb{T}_{S} S$, denoted by $h_{S}^{0}$.

Defimition 7.4. The Weil-Petersson metric on $S$ is the collection $h_{W P}^{0}:=\left\{h_{s}^{0}\right\}_{s \in S}$ of the hermitian inner product $h_{s}^{0}$ on $\mathbb{T}_{s} S$.

Thus, we have a priori two notions of $W P$ metrics $h_{W P}$ and $h_{W P}^{0}$ in this case. However, these actually coincide.

Proposition 7.3. In the Kähler-Einstein case as above, both notions coincide: $h_{W P}=$ $h_{W P}^{0}$.

Proof. This follows from Proposition 2.8 and the above definitions at once.

The proposition implies that our generalized WP metric is a correct generalization of the classical one. In what follows we write $h_{W P}$ for $h_{W P}^{0}$ and identify both. In the 
Kähler-Einstein case it is known that $h_{W P}$ is actually a $C^{\infty}$ Kähler metric on $S$ if $S$ is smooth and $X_{s}$ is in $\mathscr{A}$ for any s. (See Remark 7.2 below).

We shall generalize this result to families of extremal Kähler and Hodge manifolds with any reduced base space $S$. (See Definition 1.1 for the notion of a Kähler metric on a complex space.)

Theorem 7.4. Let $(f: \mathscr{X} \rightarrow S, \omega)$ be an effective family of extremal compact Kähler manifolds with constant scalar curvature. Suppose that $X_{s}$ is in $\mathscr{A}$ for any $s$ if $b_{1}\left(X_{s}\right)>0$. Then the generalized WP metric $h_{W P}$ is a $C^{\infty}$ Kähler metric on $S$.

Combined with Proposition 7.3, Theorem 7.4 yields:

Corollary 7.5. Let $(f: \mathscr{X} \rightarrow S, \tilde{\omega})$ be an effective family of Kähler-Einstein manifolds. Then the associated WP metric on $S$ is a $C^{\infty}$ Kähler metric.

Remark 7.2. When $S$ is nonsingular, this is due to Koiso [Koi] (with some additional conditions when $c_{1}\left(X_{s}\right) \geqq 0$ ). (See also [Sch4] for polarized families.) Note that if $c_{1}\left(X_{s}\right)>0$, then $b_{1}\left(X_{s}\right)=0$.

In the Hodge case we can weaken the assumption slightly.

Theorem 7.6. Let $(f: \mathscr{X} \rightarrow S, \tilde{\omega}, \mathscr{L})$ be an effective family of extremal Hodge manifolds with constant scalar curvature. Then the generalized WP metric $\hat{h}_{W P}$ is a $C^{\infty}$ Kähler metric on $S$.

In particular, for any compact extremal Kähler manifold $(X, \omega)$ and any extremal Hodge manifold $(X, \omega, L)$ with $X \in \mathscr{A}$, associated Kuranishi spaces $S(X, \omega)$ and $S(X, \omega, L)$ carry natural $C^{\infty}$ generalized $W P$ Kähler metrics, denoted by $h_{W P}(X, \omega)$ and $\hat{h}_{W P}(X, \omega, L)$ respectively.

In the sequel, it is also convenient to consider non-effective families. The same definitions as above give us $h_{W P}, \hat{h}_{W P}, \omega_{W P}$, and $\hat{\omega}_{W P}$ though they may be degenerate. We call $\omega_{W P}$ and $\hat{\omega}_{W P}$ generalized $W P$ form in the general case also.

We show Theorem 7.6 in a more general form.

Theorem 7.7. Let $(f: \mathscr{X} \rightarrow S, \tilde{\omega})$ be a family of extremal compact Kähler manifolds with constant scalar curvature. Let $\omega_{\mathscr{X}}$ be any admissible $(1,1)$-form for $(f, \tilde{\omega})$. Then the generalized WP form $\hat{\omega}_{W P}\left(\omega_{\mathscr{X}}\right)$ associated to $\left(f, \omega_{\mathscr{X}}\right)$ is induced by a unique element of $\Gamma\left(S, \Phi_{S}\right)$, i.e., by a unique real $C^{\infty}$ locally $\partial \bar{\partial}$-exact $(1,1)$-form on $S$.

In fact, Theorem 7.6 follows from this theorem as follows.

Proof of Theorem 7.6. For any family $(f, \tilde{\omega}, \mathscr{L})$ as in Theorem 7.6, if we take any admissible $(1,1)$-form $\omega_{\mathscr{X}}$ for $(f, \tilde{\omega})$ such that $\delta_{\mathscr{X}}\left(\omega_{\mathscr{X}}\right)=\hat{c}(\mathscr{L})$ in $H^{1}\left(\mathscr{X}, \mathscr{P}_{\mathscr{X}}\right)$ by Proposition 3.6, then by Lemma 7.1 we have $\hat{h}_{W P}=\hat{h}_{W P}\left(\omega_{\mathscr{X}}\right)$. Hence, by Theorem 7.7 the associated $W P$ form $\hat{\omega}_{W P}$ is induced by a section $\omega_{S} \in \Gamma\left(S, \Phi_{S}\right)$, which is clearly a Kähler form since $\hat{h}_{s}$ is positive definite at each point.

The proofs of Theorems 7.4 and 7.7 can be obtained by modifications of the proofs of Koiso [Koi], or of $\mathrm{Siu}$ [Si] in the Kähler-Einstein case, at least when $S$ is 
nonsingular. There is also another proof from the view point of infinite dimensional moment maps. (See [Fu5].)

Here, however, we follow a method which takes care of the cases of singular parameter spaces as well. This consists of representing the $W P$ form $\hat{\omega}_{W P}\left(\omega_{\mathscr{X}}\right)$ in Theorem 7.7 as a certain fiber integral along the fibers of $f$. To state the result we introduce a notation. Let $(f: \mathscr{X} \rightarrow S, \tilde{\omega})$ be any metrically polarized family of compact Kähler manifolds. Then $\tilde{\omega}$ induces a hermitian metric $g$ on the holomorphic relative tangent bundle $T_{\mathscr{X} / S}$, and hence a metric $\bigwedge^{n} g$ on the relative anticanonical bundle $K_{\mathscr{X} / S}^{-1}$, where $n$ is the dimension of the fibers. Then we write $c_{1}(\mathscr{X} / S, g)$ for the first chern form $c_{1}\left(K_{\mathscr{X} / S}^{-1}, \bigwedge^{n} g\right)$ and call it the relative first chern form for $(f, \tilde{\omega})$. We further set

$$
b=b(f, \tilde{\omega})=c_{1}\left(X_{s}\right) \cdot \gamma_{s}^{[n-1]} / \gamma_{s}^{[n]}, \quad \gamma_{s}=\left[\omega_{s}\right]
$$

which is independent of $s$ as long as $S$ is connected, where $\gamma^{[k]}=\gamma^{k} / k$ !

Theorem 7.8. Let $(f: \mathscr{X} \rightarrow S, \tilde{\omega})$ be a family of extremal compact $K$ ähler manifolds with constant scalar curvature with $\mathbb{S}$ connected. Let $\omega_{\mathscr{X}}$ be an admissible $(1,1)$-form for $(f, \tilde{\omega})$. Then we have the following equality:

$$
\int_{\mathscr{X} / S} c_{1}(\mathscr{X} / S, g) \omega_{\mathscr{X}}^{[n]}-b \int_{\mathscr{X} / S} \omega_{\mathscr{X}}^{[n+1]}=-1 / 2 \pi \hat{\omega}_{W P}\left(\omega_{\mathscr{X}}\right),
$$

where $\omega_{\mathscr{X}}^{[k]}=\omega_{\mathscr{X}}^{k} / k !$ for any $k$.

Remark 7.3. When $\omega_{\mathscr{X}}$ is normalized (cf. Def. 3.6), (7.2) takes a simpler form

$$
\int_{\mathscr{X} / S} c_{1}(\mathscr{X} / S, g) \omega_{\mathscr{X}}^{[n]}=-1 / 2 \pi \hat{\omega}_{W P}\left(\omega_{\mathscr{X}}\right) \text {. }
$$

Conversely, (7.2) can be deduced formally from (7.3). These fiber integral formulas are inspired by the results of [B-G-S] (cf. Sect. 10) and of Wolpert [Wo].

Theorem 7.7 is deduced from Theorem 7.8 as follows.

Proof of Theorem 7.7. The fiber integral on the left hand side of (7.2) belongs to $\Gamma\left(S, \Phi_{S}\right)$ by Varouchas (cf. Prop. 1.3). Hence $\hat{\omega}_{W P}\left(\omega_{\mathscr{X}}\right)$ is induced by an element of $\Gamma\left(S, \Phi_{S}\right)$ by Theorem 7.8. See Corollary 1.2 for the uniqueness assertion.

Thus our remaining task is to show Theorems 7.4 and 7.8. We shall prove Theorem 7.8 in the next section, and then in Section 9 Theorem 7.4 will be deduced from Theorem 7.7.

On the other hand, in the $\mathbb{K a ̈ h l e r - E i n s t e i n ~ c a s e ~ w i t h ~ n o n - z e r o ~ f i r s t ~ c h e r n ~ c l a s s ~ t h e r e ~}$ is another canonical representation of the Weil-Petersson form $\omega_{W P}$ as a fiber integral which has a more invariant form than (7.2).

Theorem 7。. Let $(f: \mathscr{X} \rightarrow \mathbb{S}, \tilde{\omega})$ be a family of Kähler-Einstein manifolds with $c_{1}\left(X_{s}\right) \neq 0$ for all $s$. Then we have

$$
r^{n} /(n+1) ! \int_{\mathscr{X} / S} c_{1}(\mathscr{X} / S, g)^{n+1}=-1 / 2 \pi \omega_{W P}
$$


where $\omega_{s} \in r c_{1}\left(X_{s}\right)$.

Proof. We set $\omega_{\mathscr{X}}=r c_{1}(\mathscr{X} / S, g)$ in (7.2). Then since $b=n / r$ in this case, we get the formula (7.4) with $\omega_{W P}$ replaced by $\hat{\omega}_{W P}$. The theorem then follows from Proposition 7.2.

Remark 7.4. When $n=1$, i.e., the case of a family of compact Riemann surfaces, this was first shown by Wolpert in [Wo] by a different method.

Finally, we draw immediate global conclusions from Theorems 7.4 and 7.6.

Theorem 7.10. Let $\mathfrak{M}_{e}$ and $\mathfrak{M}_{H, e}$ be the moduli spaces of extremal Kähler and Hodge manifolds in $\mathscr{A}$ respectively. Then there exist natural $C^{\infty} \mathrm{Kähler} \mathrm{V-metrics} \mathrm{(cf.}$ Def. 1.5) on these spaces with respect to their natural complex $V$-structures. Moreover, the natural morphism $\mu: \mathfrak{M}_{H, e} \rightarrow \mathfrak{M}_{e}$ is a Kähler V-submersion with respect to these Kähler V-metrics.

Proof. Let $(X, \omega)$ be any extremal compact Kähler manifold in $\mathscr{A}$. Since the action of the finite group $G(X, \omega):=\operatorname{Aut}(X, \omega) /$ Aut $_{o} X$ on a $\mathbb{K}$ uranishi space $S=$ $S(X, \omega)$ is induced by an automorphism of the $\mathbb{K}$ uranshi family, we see, from the functoriality, that the generalized $W P$ metric $h_{W P}(X, \omega)$ on $S$ is invariant by $G(X, \omega)$. In view of the definition of a $\mathbb{K a ̈ h l e r} V$-metric and the functoriality of $h_{W P}(X, \omega)$ it is easy to check that the collection $\left\{h_{W P}(X, \omega)\right\}$ gives a global $\mathbb{K a ̈ h l e r} V$-metric on $\mathfrak{M}_{e}$ in the sense of Definition 1.5. The proof for $\mathfrak{M}_{H, e}$ is quite the same. Finally, the last assertion follows from Proposition 9.1 below (based only on the results of Sections 2 and 4 ) and the definitions of complex $V$-structures on both the spaces.

We call the $\mathbb{K a ̈ h l e r} V$-metrics obtained in the above theorem the generalized WP $V$-metrics on $\mathfrak{M}_{H, e}$ or $\mathfrak{M}_{e}$, and the associated Kähler $V$-forms the generalized $W P$ $\mathbb{V}$-forms. The $\mathbb{K}$ ähler classes of the latter in $H^{2}(N, \mathbb{R}), N=\mathfrak{M}_{H, e}$ or $\mathfrak{M}_{e}$, (cf. Def. 1.8) is called the generalized WP class on $N$.

\section{\$ \&. A Gemerall Fiber Imategral Formula}

In this section we give a proof of the fiber integral formula (7.2), and hence of Theorem 7.8. In fact, we shall formulate and prove a more general fiber integral formula (8.2) which is true for any metrically polarized family and which specializes to (7.2) in the case of constant scalar curvature. This formula (8.2) can be proved directly starting from the set-up in Lemma 4.3 and formula (4.8). Here we proceed in a different way.

In general, let $(f: \mathscr{X} \rightarrow S, \tilde{\omega})$ be a metrically polarized family and $\omega_{\mathscr{X}}$ an admissible $(1,1)$-form for $(f, \tilde{\omega})$. Let $\xi_{s}: T_{s} S \rightarrow B_{s}^{1}$ be the linear map associated to $T^{H}\left(\omega_{\mathscr{X}}\right)$ (cf. Lemma 4.6). By the pulling-back from the hermitian space $B_{s}^{1}$ we get a natural hermitian inner product on $T_{s} S$, whose imaginary part we shall denote by $\Omega_{s}=\Omega_{s}\left(\omega_{\mathscr{X}}\right)$. Explicitly,

$$
\Omega_{s}(U, V)=\int_{X_{s}} \operatorname{tr}\left(\xi_{s}(U) \overline{\xi_{s}(V)}\right) \omega_{s}^{[n]}, \quad U, V \in T_{s} S,
$$


where $n$ is the fiber dimension of $f$. The collection $\left\{\Omega_{s}\right\}$ of $(1,1)$-forms on $T_{s} S$ on $S$ will be denoted by $\Omega_{S}=\Omega_{S}\left(\omega_{\mathscr{X}}\right)$. Since the definitions contains no harmonic projection operator it is clear that $\Omega_{S}$ is induced by a $C^{\infty}(1,1)$-form on $S$. Note that when $(f, \tilde{\omega})$ is extremal, $\xi_{s}(U)$ and $\xi_{s}(V)$ are harmonic by Theorem 4.11 ; hence $\Omega_{S}\left(\omega_{X}\right)=\hat{\omega}_{W P}\left(\omega_{X}\right)$ by the definitions. Thus, (7.2) is a consequence of the following fiber integral formula which is true for any metrically polarized family.

Theorem $\mathbb{B}_{0} \mathbb{1}_{0} \quad$ Let $(f: \mathscr{X} \rightarrow S, \tilde{\omega})$ and $\omega_{\mathscr{X}}$ be as above. Then we have

$$
\int_{\mathscr{X} / \mathbf{S}} c_{1}(\mathscr{X} / \mathbb{S}, g) \omega_{\mathscr{X}}^{[n]}-\frac{1}{2 \pi} \int_{\mathscr{X} / \mathbf{S}} R \omega_{\mathscr{X}}^{[n+1]}=-1 / 2 \pi \Omega_{S}\left(\omega_{\mathscr{X}}\right),
$$

where $R$ is the $\mathbb{R}$-valued $C^{\infty}$ function on $\mathscr{X}$ which restricts to the scalar curvature of $\omega_{s}$ on each fiber $X_{s}$. In particular, $\Omega_{S}\left(\omega_{\mathscr{X}}\right)$ is locally $\partial \bar{\partial}$-exact on $S$.

Note that the last assertion follows from Varouchas [V] and (8.2). If the family $(f, \eta)$ defined by $\left(f, \omega_{\mathscr{X}}\right)$ is effective, $\Omega_{S}\left(\omega_{\mathscr{X}}\right)$ is thus a $\mathbb{K a ̈ h l e r}$ form (cf. Lemma 4.9$)$. The left hand side of (8.2), say $A\left(\omega_{\mathscr{X}}\right)$, can also be interpreted as follows. For any real $C^{\infty}(1,1)$-form $\alpha$ on $\mathscr{X}$ we set

$$
B(\alpha):=\int_{\mathscr{X} / S} c_{1}(\mathscr{X} / S, g) \alpha^{[n]}, \quad \alpha^{[n]}=\alpha^{n} / n ! .
$$

We decompose $\omega_{\mathscr{X}}$ into the vertical and horizontal components with respect to the associated horizontal distribution $T^{H}\left(\omega_{\mathscr{X}}\right)$;

$$
\omega_{\mathscr{X}}=\hat{\omega}_{\mathscr{X}}+\omega_{\mathscr{X}}^{H},
$$

where $\hat{\omega}_{\mathscr{X}}$ and $\omega_{\mathscr{X}}^{H}$ are real $C^{\infty}(1,1)$-forms on $\mathscr{X}$ such that $\hat{\omega}_{\mathscr{X}}(U, V)=0$ whenever $U$ is horizontal, and $\omega^{H}(U, V)=0$ whenever $U$ is vertical.

Lernma 8.2. We have $A\left(\omega_{\mathscr{X}}\right)=B\left(\hat{\omega}_{\mathscr{X}}\right)$.

Proof. Denote by $c_{1}(\mathscr{X} / S, g)_{s}$ the restriction of $c_{1}(\mathscr{X} / S, g)$ to $X_{s}$. Since $\hat{\omega}_{\mathscr{X}}^{[n+1]} \equiv 0$ and $c_{1}(\mathscr{X} / S, g)_{s} \wedge \omega_{s}^{[n-1]}=1 / 2 \pi R_{s} \omega_{s}^{[n]}$ for any $s$, we get

$$
\begin{aligned}
B\left(\omega_{\mathscr{X}}\right) & =\int_{\mathscr{X} / S} c_{1}(\mathscr{X} / S, g) \hat{\omega}_{\mathscr{X}}^{[n]}+\int_{\mathscr{X} / \mathbf{S}} c_{1}(\mathscr{X} / S, g) \hat{\omega}_{\mathscr{X}}^{[n-1]} \wedge \omega_{\mathscr{X}}^{H} \\
& =B\left(\hat{\omega}_{\mathscr{X}}\right)+1 / 2 \pi \int_{\mathscr{X} / \mathbf{S}} R \hat{\omega}_{\mathscr{X}}^{[n]} \wedge \omega_{\mathscr{X}}^{H}
\end{aligned}
$$

and

$$
\int_{\mathscr{X} / \mathbf{S}} R \omega_{\mathscr{X}}^{[n+1]}=\int_{\mathscr{X} / \mathbf{S}} R \hat{\omega}_{\mathscr{X}}^{[n]} \wedge \omega_{\mathscr{X}}^{H},
$$

from which the lemma follows immediately.

Thus Theorem 8.1 is equivalent to:

Theorem 8.3. Let $(f: \mathscr{X} \rightarrow S, \tilde{\omega})$ and $\omega_{\mathscr{X}}$ be as in Theorem 8.1. Let $\hat{\omega}_{\mathscr{X}}$ be the vertical component of $\omega_{\mathscr{X}}$ defined in (8.4). Then the following equality holds true: 


$$
B\left(\hat{\omega}_{\mathscr{X}}\right):=\int_{\mathscr{X} / S} c_{1}(\mathscr{X} / S, g) \hat{\omega}_{\mathscr{X}}^{[n]}=-1 / 2 \pi \Omega_{S}\left(\omega_{\mathscr{X}}\right) .
$$

The proof depends on three lemmas. The first two give a proof of the theorem in a special situation; the general case is then considered as an approximation of this special situation.

Let $D$ be a neighborhood of the origin $o$ of $\mathbb{C}^{m}=\mathbb{C}^{m}\left(t_{1}, \ldots, t_{m}\right)$. Let $M$ be a compact connected $C^{\infty}$ manifold of even dimension $2 n$. Set $Y=D \times M$ and denote by $p: Y \rightarrow D$ the natural projection. We assume that there exists an almost complex structure on $Y$ such that $p$ is almost complex and $D(m):=D \times m$ is an almost complex submanifold for all $m \in M$. In particular, we may speak of the relative canonical bundle $K_{Y / D}$, whose restriction to each $D(m)=D$ is holomorphic line bundle.

Let now $\tilde{\omega}=\left\{\omega_{t}\right\}_{t \in D}$ be $C^{\infty}$ real $d$-closed 2-forms such that $\omega_{t}^{n}$ is a volume form on the almost complex manifold $Y_{t}:=p^{-1}(t)$. Then as a family of volume forms on $Y_{t}\left\{\omega_{t}^{n}\right\}$ is considered as a hermitian metric $h$ on $K_{Y / D}^{-1}$. Denote by $c_{1}(Y / D, h)$ the associated chern form.

Let $m$ be the ideal at $o$ of germs of $\mathbb{C}$-valued $C^{\infty}$ functions on $D$ generated by $t_{i}$, $1 \leqq i \leqq m$, and $\bar{m}$ its complex conjugate. We assume that $\dot{\omega}_{o}=0$, where the dot denotes the derivative with respect to $t$; or equivalently, we may write

$$
\omega_{t} \equiv \omega+\sum t_{\alpha} \bar{t}_{\beta} \omega_{\alpha \bar{\beta}} \quad \bmod \left(\mathrm{m}^{2}, \bar{m}^{2}\right)
$$

for some $C^{\infty}$ complex 2-forms $\omega_{\alpha \bar{\beta}}$ on $M$, where $\omega=\omega_{o}$. Denote by $\tilde{\omega}^{\prime}=\left\{\omega_{t}^{\prime}\right\}$ the constant family $\omega_{t}^{\prime}=\omega$ on $M$ and by $h^{\prime}$ and $c_{1}\left(X / S, h^{\prime}\right)$ the corresponding hermitian metrics on $K_{Y / D}^{-1}$ and the first chern form respectively.

Lemma 8.4. Set $X=Y_{o}$. Suppose that the de Rham classes of $\omega_{t}$ is independent of $t$. Then we have

$$
\int_{X} c_{1}(Y / D, h) \omega^{n}=\int_{X} c_{1}\left(Y / D, h^{\prime}\right) \omega^{n}
$$

as an element of $\bigwedge T_{o}^{*} D$, where $\int_{X}$ denotes the value of the fiber integral $\int_{\mathscr{X} / S}$ at $o$, and $\omega^{n}$ is considered as an $n$-form on Y via the natural projection $Y \rightarrow M$.

Proof. Let $m$ be any point of $M$. We compute $c_{1}(Y / D, h)$ and $c_{1}\left(Y^{\prime} / D, h^{\prime}\right)$ on $D(m)$. Let $\eta(t)$ be any non-vanishing holomorphic section of $K_{Y / D}^{-1}$ on $D(m)$. Then

$$
|\eta(t)|^{2}=p(t)|\eta(t)|^{2}
$$

where $|\eta(t)|$ and $|\eta(t)|^{\prime}$ are the norms of $\eta(t)$ with respect to the metrics $h$ and $h^{\prime}$ respectively; further $p(t)=p(t, m)$ is the restriction to $D(m)$ of the global $C^{\infty}$ function

$$
p(t, m):=\tilde{\omega}^{n} / \tilde{\omega}^{\prime n}
$$

defined on $Y$. Applying $-i / 2 \pi \partial \bar{\partial} \log$ to both sides we get on $D(m)$ the relation

$$
c_{1}(Y / D, h)=-i / 2 \pi \partial \bar{\partial} \log p(t)+c_{1}\left(Y / D, h^{\prime}\right) .
$$

On the other hand, from (8.6) we get

$$
\omega_{t}^{n} \equiv \omega^{n}+\sum t_{\alpha} \bar{t}_{\beta} v_{\alpha \bar{\beta}} \quad \bmod \left(\mathrm{m}^{2}, \bar{m}^{2}\right),
$$


where $v_{\alpha \bar{\beta}}=n \omega_{\alpha \bar{\beta}} \wedge \omega^{n-1}$ is a $C^{\infty} 2 n$-form on $M$. Further, if we define $C^{\infty}$ functions $c_{\alpha \bar{\beta}}$ on $M$ by $c_{\alpha \bar{\beta}}=v_{\alpha \bar{\beta}} / \omega^{n}$, then from (8.7) and (8.9) we get

$$
p(t) \equiv 1+\sum c_{\alpha \bar{\beta}} t_{\alpha} \bar{t}_{\beta} \quad \bmod \left(\mathrm{m}^{2}, \bar{m}^{2}\right) .
$$

Hence,

$$
\log p(t) \equiv \sum c_{\alpha \bar{\beta}} t_{\alpha} \bar{t}_{\beta} \quad \bmod \left(\mathrm{m}^{2}, \bar{m}^{2}\right)
$$

Applying $\partial \bar{\partial}$ on $D(m)$ to both sides and then evaluating at $t=0$, we have

$$
\partial \bar{\partial} \log p(t)_{\mid t=0}=\sum c_{\alpha \bar{\beta}} d t_{\alpha} \wedge d \bar{t}_{\beta} .
$$

Substituting this in (8.9) we have on $D(m)$ at $t=0$

$$
c_{1}(Y / D, h)=c_{1}\left(Y / D, h^{\prime}\right)-i / 2 \pi \sum c_{\alpha \bar{\beta}} d t_{\alpha} \wedge d \bar{t}_{\beta} .
$$

In view of the definition of $c_{\alpha \bar{\beta}}$, from this we get

$$
\int_{X} c_{1}(Y / D, h) \omega^{n}=\int_{X} c_{1}\left(Y / D, h^{\prime}\right) \omega^{n}-i / 2 \pi \int_{X} v_{\alpha \bar{\beta}} d t_{\alpha} \wedge d \bar{t}_{\beta} .
$$

But since the de $\mathbb{R}$ ham classes of $\omega_{t}$ are constant, $\omega_{\alpha \bar{\beta}}$, and so $v_{\alpha \bar{\beta}}$ also, must be $d$-exact. Hence the last term of (8.10) vanishes. The lemma follows.

Retaining the previous notations we assume for simplicity that $\mathbb{X}:=\mathbb{Y}_{o}$ is a complex manifold. Let $A^{1}=A^{1}(X)=\Gamma\left(X, T \otimes \bar{T}^{*}\right)$ as before. Then we may represent the family of almost complex structures on $Y_{t}$ canonically by a $C^{\infty}$ family $\tilde{\varphi}=\left\{\varphi_{t}\right\}$ of elements of $A^{1}$ (cf. §4). For any vector $v \in \mathbb{T}_{o} D$ one can take the derivative $\mathbb{L}_{v} \tilde{\varphi} \in A^{1}$ at $o$ of this family. Denote by $\xi_{0}$ the resulting linear map $\xi_{0}: \mathbb{T}_{o} D \rightarrow A^{1}, \xi_{0}(v)=\mathbb{L}_{v} \tilde{\varphi}$. Then we get an element $\Omega_{0}$ of $\bigwedge^{1,1} \mathbb{T}_{o}^{*} \mathbb{D}$ by

$$
\Omega_{0}(u, v)=\int_{X} \operatorname{tr}\left(\xi_{0}(u) \overline{\xi_{0}(v)}\right) \omega^{[n]} \quad u, v \in \mathbb{T}_{o} D
$$

where elements of $A^{1}$ is regarded as a map $\bar{T} \rightarrow \mathbb{T}$ and tr is taken in End $\mathbb{T}$.

Lemma \$.5. In the above notations we have

$$
\int_{X} c_{1}\left(Y / S, h^{\prime}\right) \omega^{[n]}=-1 / 2 \pi \Omega_{o}
$$

in the same convention as in the previous lemma.

Proof. Fix any $m \in M$. Then $D=D(m) \rightarrow \operatorname{Hom}\left(\bar{T}_{x}, T_{x}\right)=\operatorname{Hom}\left(T_{x}^{*}, \bar{T}_{x}^{*}\right), x \rightarrow$ $\varphi_{t}(x)$, is a holomorphic map into a finite dimensional vector space, where $x=(o, m)$. Take any $\mathbb{C}$-basis $e_{1}, \ldots, e_{n} \in \mathbb{T}_{x}^{*}$. Then

$$
\mu(t):=\bigwedge_{1}^{n}\left(e_{i}+\varphi_{t}\left(e_{i}\right)\right)
$$

gives a nonvanishing holomorphic section of $K_{Y / D}$ on $\mathbb{D}(m)$. Then the norm $|\mu(t)|$ of $\mu(t)$ with respect to $h$ is given by: 


$$
\mu(t) \wedge \bar{\mu}(t)=|\mu(t)|^{2} \mu(o) \wedge \bar{\mu}(o) .
$$

From this we get that if we set

$$
B(t)=\left(\begin{array}{cc}
I & \bar{\varphi}_{t} \\
\varphi_{t} & I
\end{array}\right) \in \operatorname{End}\left(T_{x}^{*} \oplus \bar{T}_{x}^{*}\right)
$$

we have $|\mu(t)|^{2}=\operatorname{det} B(t)=\operatorname{det}\left(I-\varphi_{t} \bar{\varphi}_{t}\right)$, where $I$ denotes the identity. Hence we have

$$
\begin{aligned}
c_{1}\left(Y / S, h^{\prime}\right) & =i /\left.2 \pi \partial \bar{\partial} \log |\mu(t)|^{2}\right|_{t=0} \\
& =-1 / 2 \pi \operatorname{tr}\left(\partial_{t} \varphi_{t}(o) \bar{\partial}_{t} \bar{\varphi}_{t}(o)\right),
\end{aligned}
$$

where $\partial_{t} \varphi_{t}(o)$ is the symbolical expression for $\xi_{0}$. Hence, by taking the fiber integral with respect to the volume form $\omega^{[n]}$ yields the lemma.

In order to realize the situation of the above lemmas in the situation of Theorem 8.2 we recall a result of Kuranishi $[\mathrm{Ku}]$. Let $f: \mathscr{X} \rightarrow S$ be as in Theorem 8.2. Then a $C^{\infty}$ trivialization $\psi: \mathscr{X} \rightarrow S \times M$ of $f$ is said to depend holomorphically on $s \in S$ if for any point $m \in M, S_{1}(m):=\psi^{-1}(S \times m)$ is an analytic subset of $\mathscr{X}$. In this case $T^{H} \psi:=\bigcup T_{x}^{H} \psi, T_{x}^{H}(\psi)=T_{x} S_{1}(m)$, with $\psi(x)=(f(x), m)$, is the horizontal subbundle associated to $\psi$.

Lemma 8.6. Let $o \in S$ be any point. Suppose that we are given a $C^{\infty}$ complex horizontal subbundle $T^{H}$ for $f$. Then there exists a $C^{\infty}$ trivialization $\psi$ of $f$ depending holomorphically on such that $T^{H} \psi\left|X=T^{H}\right| X$.

Proof. The result is due to Kuranishi $[\mathrm{Ku}]$ if we neglect the additional tangential condition. However, as the proof there shows it is immediate to see that the construction can be carried out with the given initial data $T \mathscr{X}\left|X=T^{H}\right| X \oplus T$.

Proof of Theorem 8.3. Fixing an arbitrary point $o \in S$ we show the equality $B\left(\hat{\omega}_{\mathscr{X}}\right)=-1 / 2 \pi \Omega_{S}\left(\omega_{\mathscr{X}}\right)_{o}$ as an element of $\bigwedge^{1,1} T_{o}^{*} S$. By Lemma 8.6 there exists a $C^{\infty}$

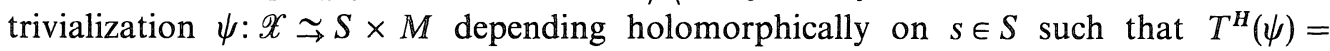
$T^{H}\left(\omega_{\mathscr{X}}\right)$ along $X$. We may assume that $S$ is an analytic subset of a domain $D$ in $\mathbb{C}^{m}, o$ is the origin of $\mathbb{C}^{m}$, and $T_{o} S=T_{o} D$. By $\psi$ we consider $\tilde{\omega}=\left\{\omega_{s}\right\}_{s \in S}$ as a $C^{\infty}$ family of symplectic forms on $M$ with a fixed de Rham class; $\psi$ then defines a $C^{\infty}$ family of Kähler structures $\tilde{J}=\left\{J_{s}\right\}_{s \in S}$ on $\left(M, \omega_{s}\right)$. We may assume further that $\tilde{J}$ and $\tilde{\omega}$ extends to a $C^{\infty}$ family $\left\{J_{t}\right\}$ and $\left\{\omega_{t}\right\}, t \in D$, such that $\omega_{t}$ are $d$-closed with fixed de Rham class and $J_{t}$ depends holomorphically on $t$ also. Hence on $Y:=D \times M$ we get a natural almost complex structure for which the projection $p: Y \rightarrow D$ is almost holomorphic and $D(m), m \in M$, are almost complex submanifold of $Y$. Finally by Lemma 4.5 the condition (8.6) is fulfilled. Therefore, all the conditions of Lemmas 8.4 and 8.5 are satisfied. The theorem then follows from these two lemmas.

\section{§9. Comparison of Generalized Weil-Petersson Metrics and Proof of Theorem 7.4}

In this section first we compare two generalized Weil-Petersson metrics $\hat{h}_{W P}$ and $h_{W P}$ for Hodge manifolds. In global terms our results says that 1 ) the natural morph- 
ism $\mu: \mathfrak{M}_{H, e} \rightarrow \mathfrak{M}_{e}$ is a $\mathbb{K}$ ähler $V$-submersion, 2) $\mathfrak{M}_{H, e}$ admits a natural foliation which is transversal to the fibers of $\mu, 3)$ the generalized WP metric is $V$-flat on each fiber, 4 ) the generalized $W P V$-form on $\mathfrak{M}_{e}$ is a fiber integral of a power of that on $\mathfrak{M}_{H, e}$, and 5) the locus of canonical bundles on $\mathfrak{M}_{H, e}$ is a union of leaves of the foliation in 2). As an application of these considerations we also prove Theorem 7.4 in the second half of this section.

We consider it locally. Let $(X, \omega, L)$ be an extremal Hodge manifold in $\mathscr{A}$. Let $\tilde{S}:=S(X, \omega, \mathbb{L})$ and $S:=S(X, \omega)$ be $\mathbb{K}$ uranishi spaces of $(X, \omega, \mathbb{L})$ and $(X, \omega)$ with generalized WP metrics $\hat{h}_{W P}$ and $h_{W P}$ respectively. In this section we first study a relation between $\hat{h}_{W P}$ and $h_{W P}$ with respect to the natural smooth morphism $r: \tilde{S} \rightarrow S$. This will then give a proof of Theorem 7.4 immediately in this case (cf. Corollary 9.3 below) and also give us the idea how to proceed in the general case.

Recall that $\hat{h}_{W P}$ is a $C^{\infty} \mathbb{K a ̈ h l e r}$ metric by Theorem 7.6. Hence, it defines a $\mathbb{C}^{\infty}$ horizontal distribution $T^{H} \tilde{S}:=T^{H}\left(\hat{\omega}_{W P}\right)$. Recall that $r$ is said to be a Kähler submersion if for any $\tilde{s} \in \tilde{S}$ with $s=r(\tilde{S})$ the induced isomorphism $r_{*}: \mathbb{T}_{\tilde{s}}^{H} \tilde{S} \rightarrow T_{s} S$ is isometric with respect to the hermitian metrics $\hat{h}_{W P, \tilde{s}} \mid T_{\tilde{s}}^{H} \tilde{S}$ and $h_{W P, s}$ (cf. Def. 1.3).

Proposition 9.1. 1) $r$ is a Kähler submersion. 2) The restriction of $\hat{h}_{W P}$ to each fiber of $r$ is flat.

Proof. 1) We first observe the following commutative diagram which we get from (4.4) and Theorems 2.5 and 2.9:

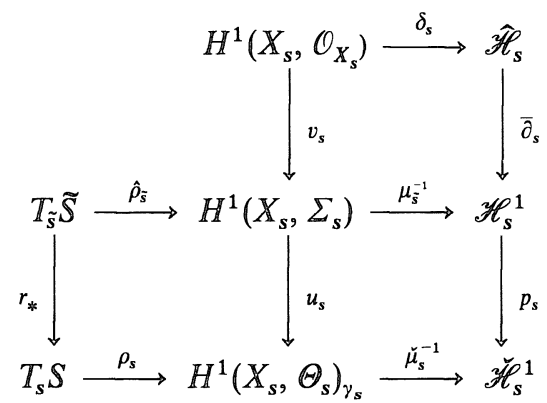

where $\Sigma_{s}=\Sigma_{L_{s}}=\Sigma_{\gamma_{s}}$, $\hat{\mathscr{H}}_{s}=\hat{\mathscr{H}}_{B_{s}}$, and $p_{s}$ is the orthogonal projection. The assertion then follows from this, the fact that $\hat{\rho}_{\tilde{s}}\left(T_{\tilde{s}}{\widetilde{S_{s}}}_{s}\right)=\operatorname{Im} v_{s}$, and the definitions of $\check{\mathscr{H}}_{s}^{1}, \hat{h}_{W P}$ and $h_{W P}$. Here, $\tilde{S_{\tilde{s}}}=r^{-1}(s)$.

2) For simplicity of notations we show 2) only for the fiber $F$ through $\tilde{o} \in \tilde{S}$, where $\tilde{o}$ is the base point of $\tilde{S}$. (The proof in the general case is completely the same since $X$ is in $\mathscr{A}$.) Let $l \in \mathbb{P i c} X$ be the point corresponding to $L$. Take an abelian subvariety $T$ of Pic $X$ passing through $l$ which is transversal to the Aut $_{o} X$-orbit $O$ of $l$ in Pic $X$ with $\operatorname{dim} T+\operatorname{dim} O=\operatorname{dim} \operatorname{Pic} X$. Let $\mathscr{L}$ be the restriction of a universal line bundle on $X \times \operatorname{Pic} X$ to $X \times T$. Then the universal morphism $T \rightarrow \tilde{S}$ associated to the family $\left(p: X \times T \rightarrow T, \tilde{\omega}_{0}, \mathscr{L}\right)$ of extremal Hodge manifolds induces an isomorphism $(T, l) \cong(\tilde{S}, \tilde{o})$, where $p$ is the natural projection and $\tilde{\omega}_{0}=\{\omega\}$ is the constant family.

So we have only to show the flatness of the generalized WP metric for the extremal family $\left(p, \tilde{\omega}_{0}, \mathscr{L}\right)$. By our construction the tangent space of $T$ at $t \in T$ is identified via 
the Kodaira-Spencer map with the image $I$ of the homomorphism $v: H^{1}\left(\mathcal{O}_{\mathscr{X}}\right) \rightarrow H^{1}\left(\Sigma_{L}\right)$, independently of $t$. Thus it suffices to show that the hermitian metric on $I$ induced by the isomorphism $\mu_{t}^{-1}: H^{1}\left(\Sigma_{L}\right) \widetilde{H_{B}^{1}}$ of (2.15) actually is independent of $t$, or more specifically, that $\mu_{t}^{-1} v$ is independent of $t$. In fact, this can be seen from the following commutative diagram (cf. Theorem 2.9):

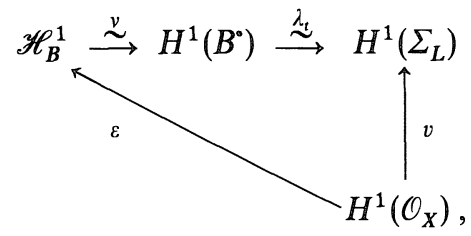

where $\mu_{t}=\lambda_{t} v$, and $\varepsilon$ and $v$ are independent of $t \in T$.

By 1) of the above proposition and a theorem of Watson [Wa] if we restrict $T^{H} \tilde{S}$ to the set $\tilde{S}_{\text {reg }}$ of smooth points, then $T^{H} \tilde{S}$ is integrable with each leaf $F$ a complex submanifold. $F$ turns out to extend across the singularities of $\tilde{S}$.

Proposition 9.2. The closure $\bar{F}$ in $\tilde{S}$ of each leaf $F$ as above is an analytic subset of $\tilde{S}$ which is mapped isomorphically onto $S$ by $r$. Moreover, the Zariski tangent space $T_{\tilde{s}} \bar{F}$ at each point $\tilde{s} \in \bar{F}$ coincides with $T_{\tilde{s}}^{H} \tilde{S}$.

Corollary 9.3. For any $\bar{F}$ above, $r$ induces a biholomorphic Kähler isometry $(\bar{F}$, $\left.\left.\hat{h}_{W P}\right|_{\bar{F}}\right) \cong\left(S(X, \omega), h_{W P}\right)$. In particular, the generalized $W P$ metric $h_{W P}$ on $S(X, \omega)$ is a $C^{\infty}$ Kähler metric.

Proof. The first assertion is immediate from the above two propositions. Then, since $\hat{h}_{W P}$ is a $C^{\infty}$ Kähler metric, the same is also true for $h_{W P}$.

The last assertion of the corollary is exactly the statement of Theorem 7.4 (for $S(X, \omega))$ in the Hodge case. Before proceeding to the proof of the proposition we make an observation related with the above result in the case $L=K_{X}^{m}, m \in \mathbb{Z}-0$. In this case we have a natural holomorphic section $\sigma: S \rightarrow \tilde{S}$ with $\sigma(o)=\tilde{o}$ of $r$, i.e., the universal morphism associated to the family $\left(f: \mathscr{X} \rightarrow S, \tilde{\omega}, K_{\mathscr{X} / S}^{m}\right)$ of extremal Hodge manifolds, where $(f, \tilde{\omega})$ is a Kuranish family of $(X, \omega)$.

Proposition 9.4. The image $\sigma(S)$ coincides with the leaf $\bar{F}$ passing through of the horizontal distribution $T^{H} \tilde{S}$ as in Proposition 9.2.

Proof. Let $\tilde{s}$ be any point of $\tilde{S}$ with $r(\tilde{s})=s$. By Proposition 9.1 and Proposition

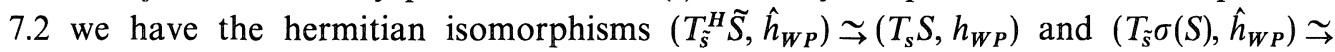
$\left(T_{s} S, h_{W P}\right)$ respectively, both induced by $r_{*}$. This implies that the orthogonal projection $T_{\tilde{s}} \tilde{S} \rightarrow T_{\tilde{s}}^{H} \tilde{S}$ restricted to the subspace $T_{\tilde{s}} \sigma(S)$ is an isometry. This implies that $T_{\tilde{s}}^{H} \tilde{S}=$ $T_{\tilde{s}} \sigma(S)$.

As an application of Proposition 9.1 we also give a formula which essentially gives the generalized $W P V$-form on $\mathfrak{M}_{e}$ as a fiber integral of a power of that on $\mathfrak{M}_{H, e}$. Let $(X, \omega)$ be an extremal Kähler manifold with integral Kähler class $[\omega]$. We recall the commutative diagram (6.6): 


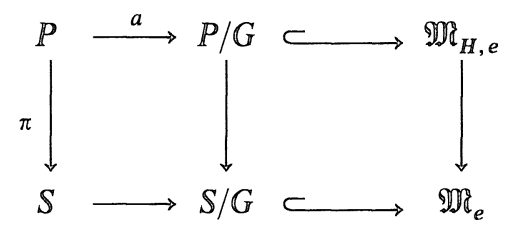

where $P=P_{\gamma}(\mathscr{X} / S), S=S(X, \omega)$ and $G=G(X, \omega)$; further $a$ is a $V$-morphism into $\mathfrak{M}_{H, e}$. So we get a lift $\bar{\omega}_{W P}:=a^{*} \omega_{W P}\left(\mathfrak{D U}_{H, e}\right)$ of the generalized $W P \mathbb{P}$-form $\omega_{W P}\left(\mathfrak{M U}_{H, e}\right)$ on $\mathfrak{M}_{H, e}$ to an (ordinary) $\mathbb{K} a ̈ h l e r$ form on $P$.

Proposition 9.5. Let $m$ be the dimension of the fibers of $\pi$. Let $C=\int_{P_{s}} \bar{\omega}_{W P}(s)$, which is independent of $s$. Then we get on $S=S(X, \omega)$ the fiber integral formula

$$
\int_{P / S} \bar{\omega}_{W P}^{m+1}=(m+1) C \omega_{W P}
$$

where $\omega_{W P}=\omega_{W P}(X, \omega)$.

Proof. Decompose $\bar{\omega}_{W P}$ into vertical and horizontal components with respect to $T^{H}\left(\bar{\omega}_{W P}\right) ; \bar{\omega}_{W P}=\hat{\bar{\omega}}_{W P}+\bar{\omega}_{W P}^{H} . \quad$ Then we get

$$
\int_{P / S} \bar{\omega}_{W P}^{m+1}=(m+1) \int_{P / S} \hat{\bar{\omega}}_{W P}^{m} \wedge \omega_{W P}^{H}=(m+1) C \omega_{W P}
$$

as desired, where the last equality follows from Proposition 9.1, 1).

Now we are in a position to prove Proposition 9.2.

Proof of Proposition 9.2. In the notations of Proposition 5.11 let $Q$ be the connected component of $P_{\gamma}(\mathscr{X} / S)$ which contains $\bar{l}$. This is a smooth fiber space of abelian varieties over $S . \quad \mathbb{B} y$ the definition of $\bar{\omega}_{W P},\left((Q, \bar{l}), \bar{\omega}_{W P}\right)$ and $\left((\tilde{S}, \tilde{o}), \hat{\omega}_{W P}\right)$ are isomorphic over $S$ (cf. Proposition 5.11). Hence, it suffices to prove the corresponding assertion for the fiber space $\pi:\left(Q, \bar{\omega}_{W P}\right) \rightarrow\left(S, \omega_{W P}\right)$. Let $T^{H} Q=T^{H}\left(\bar{\omega}_{W P}\right) . \quad\left(Q, \bar{\omega}_{W P}\right)$ is a $\mathbb{K a ̈ h l e r}$ space with $\pi$ a $\mathbb{K a ̈ h l e r ~ s u b m e r s i o n ; ~ f u r t h e r m o r e , ~ o n ~ e a c h ~ f i b e r ~} \bar{\omega}_{W P}$ induces a flat $\mathbb{K}$ ähler form $\bar{\omega}_{s}$ on an abelian variety, and on the smooth locus $\mathcal{Q}_{\text {reg }}$ of $Q, T^{H} Q$ is integrable and each leaf, say $F$, is a complex submanifold of $Q_{\text {reg }}$. We have to show that the closure $\bar{F}$ of $F$ in $Q$ is an analytic subspace which is mapped isomorphically onto $S$ and that $T_{q} \bar{F}=T_{q}^{H} Q$ for any $q \in \bar{F}$.

Now, considered as an extremal deformation of $\left(Q_{o}, \omega_{o}\right)$, where $\omega_{o}=\bar{\omega}_{W P} \mid Q_{o}, \pi$ is induced from a $\mathbb{K}$ uranishi family $\left(\pi_{0}: Q_{0} \rightarrow S_{0}, \tilde{\omega}_{0}\right)$ of $\left(Q_{o}, \omega_{o}\right)$, where $S_{0}$ is smooth. For $\left(\pi_{0}, \tilde{\omega}_{0}\right)$ from its construction it is immediate to see that we have an admissible $(1,1)$-form $\omega_{Q_{0}}$ such that the corresponding horizontal distribution $T^{H} Q_{0}$ is integrable and each leaf is a complex submanifold mapped isomorphically to $S_{0}$.

Let $\bar{\omega}_{Q}$ be the pull-back of $\omega_{Q_{0}}$ to $Q$ by the given morphism $u: Q \rightarrow Q_{0}$. Then by Proposition 3.9 there exists an automorphism $g$ of $Q$ over $S$ such that $g^{*} \bar{\omega}_{Q}$ and $\bar{\omega}_{W P}$ are equivalent. Therefore, by Lemma 3.8 we have only to prove the corresponding assertion for $g^{*} \bar{\omega}_{Q}$. But the closure of each leaf of $T^{H}\left(g^{*} \bar{\omega}_{Q}\right)$ on $Q_{\text {reg }}$ is just the inverse image of some leaf on $Q_{0}$ of $\mathbb{T}^{H}\left(\omega_{Q_{0}}\right)$ via $u g$. Hence, for $g^{*} \bar{\omega}_{Q}$ the assertion is immediate to see. 
As the above proof and Corollary 9.3 indicate, even in the general case, if we construct a family of complex tori over $s$ which has the same property as $Q \rightarrow S$ in the above proof, Theorem 7.4 could be deduced from Theorem 7.7 in the same manner. We first construct such a fiber space.

Let $(X, \omega)$ be an extremal compact $\mathbb{K} a ̈ h l e r$ manifold in $\mathscr{A}$. Let $S=S(X, \omega)$ be a Kuranishi space of $(X, \omega)$ and $(f: \mathscr{X} \rightarrow S, \tilde{\omega})$ the associated $\mathbb{K}$ uranishi family.

Proposition 9.6. There exist a family of complex tori $\pi: Q \rightarrow S$ over $S$ and an admissible $(1,1)$-form $\omega_{\mathscr{Z}}$ for the induced family $\left(\bar{f}: \mathscr{Z} \rightarrow Q, \tilde{\omega}_{Q}\right), \mathscr{Z}=\mathscr{X} \times{ }_{S} Q$, with the following properties: 1) If we set $\eta_{\mathscr{Z}}=\delta_{0}\left(\omega_{\mathscr{Z}}\right) \in\left(R^{1} \bar{f}_{*} \mathscr{P}_{\mathscr{Z}}\right)(Q)$, then for any point $q \in Q$ the Kodaira-Spencer map $\hat{\rho}_{q}: T_{q} Q \rightarrow H^{1}\left(X_{s}, \Sigma_{s}\right)$ associated to $\left(\bar{f}, \eta_{\mathscr{Z}}\right)$ (cf. Def. 4.1) is injective and induces an isomorphism $T_{q} Q_{s} \underset{\rightarrow}{\rightarrow}$, where $s=\pi(q), Q_{s}=\pi^{-1}(s)$, and $I_{s}$ is the image of the natural homomorphism $v_{s}: H^{1}\left(X_{s}, \mathcal{O}_{X_{s}}\right) \rightarrow H^{1}\left(X_{s}, \Sigma_{s}\right)$. 2) Let $\hat{h}_{W P}=\hat{h}_{W P}\left(\omega_{\mathscr{Z}}\right)$ be the generalized WP metric associated to $\left(\bar{f}, \tilde{\omega}_{Q}, \eta_{\mathscr{Z}}\right)$, or equivalently to $\left(\bar{f}, \omega_{\mathscr{Z}}\right)$. Then the map $\pi:\left(Q, \hat{h}_{W P}\right) \rightarrow\left(S, h_{W P}\right)$ is a Kähler submersion and the restriction of $\hat{h}_{W P}$ to each fiber of $\pi$ is a flat Kähler metric.

Proof. a) Construction of the fiber space $Q \rightarrow S$. Since $\mathbb{R}^{1} f_{*} \mathcal{O}_{\mathscr{X}}$ is locally free on $S$ and commutes with base changes, there exists holomorphic vector bundle $V$ on $S$ such that for any base change $u: S^{\prime} \rightarrow S$ we have a canonical isomorphism $\mathcal{O}_{S^{\prime}}\left(u^{*} V\right) \cong$ $R^{1} f_{*}^{\prime} \mathcal{O}_{\mathscr{X}^{\prime}}$, where $\mathscr{X}^{\prime}=\mathscr{X} \times{ }_{S} S^{\prime}$ and $f^{\prime}: \mathscr{X}^{\prime} \rightarrow S^{\prime}$ is an induced morphism. In particular, we have the identification $V=\cup_{s} H^{1}\left(X_{s}, \mathcal{O}_{X_{s}}\right)$. The natural sheaf homomorphism $R^{1} f_{*} \mathbb{R} \rightarrow R^{1} f_{*} \mathcal{O}_{\mathscr{X}}$ induces isomorphisms $H^{1}\left(X_{s}, \mathbb{R}\right) \rightarrow H^{1}\left(X_{s}, \mathcal{O}_{X_{s}}\right)$ over the reals; so we may consider also

$$
V=\bigcup_{s} H^{1}\left(X_{s}, \mathbb{R}\right)
$$

as a family of real vector spaces.

Similarly, $f_{*} \Theta_{\mathscr{X} / S}$ is locally free and commutes with base changes since $X_{s}$ is in $\mathscr{A}$; so we get a holomorphic vector bundle $U \rightarrow S$ with similar properties for $f_{*} \Theta_{\mathscr{X} / S}$; moreover, the natural homomorphism $f_{*} \Theta_{\mathscr{X} / S} \rightarrow R^{1} f_{*} \mathcal{O}_{\mathscr{X}}$ arising from (4.2) is injective and its cokernel is locally free; hence it is realized by a vector bundle embedding $U \rightarrow V$. Take any holomorphic subbundle $\mu: W \rightarrow S$ of $V \rightarrow S$ such that 1) $U \oplus W \widetilde{\rightarrow}$, and 2) there exists a local subsystem $N$ of $R^{1} f_{*} \mathbb{R}$ such that we have $W=\cup_{s} N_{s}$ in the identification (9.3). Take then a discrete local subsystem $\Gamma$ of $N$ such that $Q:=W / \Gamma$ is proper over $S$. Then $Q$ is a fiber space of complex tori over $S$.

b) Construction of a refined Kähler polarization $\eta_{\mathscr{Z}}$ : We set $\mathscr{Y}=\mathscr{X} \times{ }_{S} W$ and let $\tilde{f}: \mathscr{Y} \rightarrow W$ be the natural morphism. We consider the tautological section $\sigma$ of $R^{1} \tilde{f}_{*} \mathcal{O}_{\mathscr{y}}$; namely with respect to the canonical isomorphisms

$$
R^{1} \tilde{f}_{*} \mathcal{O}_{\mathscr{y}} \cong \mathcal{O}_{W}\left(\mu^{*} V\right) \cong \mathcal{O}_{W}\left(V \times_{S} W\right) \supseteqq \mathcal{O}_{W}\left(W \times_{S} W\right),
$$

$\sigma$ corresponds to the diagonal section of $W \times{ }_{S} W$.

We fix a section $\eta_{\mathscr{X}}$ of $R^{1} f_{*} \mathscr{P}_{\mathscr{X}}$ which is mapped to $\tilde{\gamma}=\delta_{2} \delta_{1}(\tilde{\omega})$ with respect to the natural homomorphism $R^{1} f_{*} \mathscr{P}_{\mathscr{X}} \rightarrow R^{2} f_{*} \mathbb{R}$. By pulling it back to $W$, we obtain a section $\eta_{y}^{0}$ of $R^{1} \tilde{f}_{*} \mathscr{P}_{y}$ over $\mathbb{W}$. We then set

$$
\eta_{\mathscr{Y}}=\eta_{\mathscr{y}}^{0}+\alpha(\sigma)
$$


where $\alpha$ is the natural homomorphism $\mathbb{R}^{1} \tilde{f}_{*} \mathcal{O}_{\mathscr{y}} \rightarrow \mathbb{R}^{1} \tilde{f}_{*} \mathscr{P}_{\mathscr{y}}$.

Any section $\beta: S \rightarrow \Gamma \subseteq N$, considered as a holomorphic section of $W$, induces by translation an automorphism $\beta^{*}$ of $W$ over $S$, and then of $\tilde{f}: \mathscr{Y} \rightarrow \mathbb{W}$ and also of the sheaf $R^{1} \tilde{f}_{*} \mathscr{P}_{\mathscr{y}}$. But in view of the exact sequence $\left(\mu^{*} N \subseteq\right) \mathbb{R}^{1} \tilde{f}_{*} \mathbb{R} \rightarrow \mathbb{R}^{1} \tilde{f}_{*} \mathcal{O}_{\mathscr{Y}} \rightarrow$ $R^{1} \tilde{f}_{*} \mathscr{P}_{\mathscr{y}}, v^{*}$ leaves fixed the section $\eta_{\mathscr{y}}$ in the last action. Hence $\eta_{\mathscr{Y}}$ descends to a section $\eta_{\mathscr{Z}}$ of $R^{1} \bar{f}_{*} \mathscr{P}_{\mathscr{Z}}$.

c) We take any admissible $(1,1)$-form $\omega_{\mathscr{Z}}$ which is mapped to $\eta_{\mathscr{Z}}$ for $\left(f, \tilde{\omega}_{Q}\right)$. (See Proposition 3.6.) We check first the condition 1). For any point $q \in \mathbb{Q}$ with $s=\pi(q)$, by our construction $T_{q} Q_{s}$ is naturally identified with the subspace of $H^{1}\left(X_{s}, \mathcal{O}_{X_{s}}\right)$, and then by the definition of $\eta_{\mathscr{Z}}, \hat{\rho}_{q}$ restricted to $T_{q} Q_{s}$ is induced by the canonical homomorphism $v_{s}: H^{1}\left(\mathcal{O}_{X_{s}}\right) \rightarrow H^{1}\left(\Sigma_{s}\right)$ and is isomorphic onto the image of $v_{s}$ by our choice of $W$. Since $S$ is a $\mathbb{K}$ uranishi space, it follows that $\hat{\rho}_{q}$ itself is injective.

Finally, the first property of 2 ) is verified in the same way as for 1) of Proposition 9.1 by using the diagram (9.1), and the second property also is proved in the same way as the second half of the proof of 2) of Proposition 9.1 replacing $\Sigma_{L}$ by $\Sigma_{\gamma}$ in (9.2).

Proof of Theorem 7.4. The case $b_{1}=0$ follows from Remark 7.1 and Theorem 7.7 as in the proof of Theorem 7.6. So we may assume that $X_{s} \in \mathscr{A}$ for all $s$. In this case, to the fiber space $\pi:\left(Q, \hat{h}_{W P}\right) \rightarrow\left(S, h_{W P}\right)$ in Proposition 9.6 we can apply the proof of Proposition 9.2 (instead of to $\left(Q, \bar{\omega}_{W P}\right) \rightarrow\left(S, \omega_{W P}\right)$ there). As a conclusion we get that the horizontal distribution $T^{H} Q:=T^{H}\left(\hat{\omega}_{W P}\right)$ is integrable so that for each point $q \in Q$ there exists an (analytic) leaf $\bar{F}$ passing through $q$ which is mapped isomorphically to $S$ and $T_{q} \bar{F}=T_{q}^{H} Q$. Finally, the theorem follows from the $\mathbb{K a ̈ h l e r}$ isometry $\left(F, \hat{h}_{W P} \mid \bar{F}\right) \simeq$ $\left(S, h_{W P}\right)$ as in Corollary 9.3 because of the universality of $S(X, \omega)$.

\section{§10. Determinamt Lime Bumdles, Quillen Metries, and their Applications}

In this section, combining our fiber integral formula (Theorem 7.8) and the main result of $[\mathrm{B}-\mathrm{G}-\mathrm{S}]$ we construct a hermitian line bundle $(F, k)$ on the base space of any family $(f: \mathscr{X} \rightarrow S, \tilde{\omega}, \mathscr{L})$ of extremal Hodge manifolds whose first chern form gives up to a constant the associated generalized Weil-Petersson form. Thus $F$ is obtained as a certain determinant line bundle associated to a virtual vector bundle on $\mathscr{X}$ and hence is canonically determined: $k$ is the Quillen metric depending on the choice of an admissible hermitian metric on $\mathscr{L}$ for $(f, \tilde{\omega})$. In the $\mathbb{K}$ ähler-Einstein case $k$ also can be chosen canonical.

When we deal with families with singular parameter spaces we need [B-G-S] with singular parameter spaces also. In this respect we have formulated such a generalization also in this section, the proof of which will be given in Section 12.

We shall first recall the main result of [B-G-S]. Let $X$ be a compact $\mathbb{K}$ ähler manifold with a fixed Kähler metric $g$. Let $E$ be a holomorphic vector bundle on $X$ with a $C^{\infty}$ hermitian metric $h$. Then we define a 1 -dimensional complex vector space $\lambda(E)$ by

$$
\lambda(E)=\lambda(X, E):=\bigotimes_{q \geqq 0}\left(\bigwedge^{\max } H^{q}(X, E)\right)^{(-1)^{q+1}},
$$


where $\bigwedge^{\max }$ denotes in general the highest exterior power. Let $\mathscr{H}^{q}(E)$ be the space of harmonic forms on $X$ with values in $E$ with respect to the metrics $g$ and $h$. This is naturally a hermitian vector space. Hence, the natural isomorphism $\mathscr{H}^{q}(E) \cong H^{q}(X, E)$ induces a natural hermitian metric on each $H^{q}(X, E)$, and hence on $\lambda(E)$ also. We denote by ' $k$ the hermitian metric on $\lambda(E)$ thus obtained.

Further, one associates to any pair $Q=((X, g),(E, h))$ as above a real number $\tau=\tau(Q)$ as follows. Let $\left(D^{\circ}(E), \bar{\partial}\right)$ be the Dolbeault complex associated to $E$. Then, letting $\bar{\partial}^{*}$ be the formal adjoint of $\bar{\partial}$ with respect to $g$ and $h$ we consider the Laplacian $\square_{q}=\overline{\partial \partial}^{*}+\bar{\partial} * \bar{\partial}$ operating on $D^{q}(E)$. Then the associated zeta function $\zeta_{q}(s)=\Sigma \lambda^{-s}$, where the summation is over all the nonzero eigenvalues $\lambda$ of $\square_{q}$ counted with multiplicities, converges absolutely if $\operatorname{Re} s$ is sufficiently large and has a meromorphic continuation to the whole complex plane $\mathbb{C}$. Moreover, it is holomorphic at the origin so that its derivative $\zeta_{q}^{\prime}(o)$ at $o$ makes sense. Then the analytic torsion $\tau$ is defined by

$$
\tau=\sum_{q \geqq 0}(-1)^{q} q \zeta_{q}^{\prime}(o)
$$

Now by using $\tau$ we define a new hermitian metric $k$ on $\lambda(E)$ by

$$
k=k_{E}:={ }^{\prime} k e^{-(1 / 2) \tau} .
$$

Then $k$ is called the Quillen metric of $\lambda(E)$ associated to the pair $Q$.

We next consider the relative version of the above construction. Let $(f: \mathscr{X} \rightarrow S, \tilde{\omega})$ be a metrically polarized family of compact Kähler manifolds and $E \rightarrow \mathscr{X}$ a holomorphic vector bundle. First, by $\left[\mathrm{F}-\mathrm{K} ; \mathrm{Th}\right.$. II] the direct image $R f_{*} \mathscr{E}$ (in the derived category) is a perfect complex, i.e., locally on $S$ there exists a bounded complex $\mathscr{L}^{\bullet}$ of finite free $\mathcal{O}_{S}$-modules which is quasi-isomorphic to $R f_{*} \mathscr{E}$. (We denote by $\mathscr{E}$ the locally free sheaf corresponding to $E$.) Then the theory of Knudsen-Mumford [K-M] associates with $R f_{*} \mathscr{E}$ canonically an invertiblae $\mathcal{O}_{S}$-module det $R f_{*} \mathscr{E}$, called the determinant of $R f_{*} \mathscr{E}$. (Most of the theory of [K-M] also works in the analytic category.) Then we define $\lambda(E)$ to be the holomorphic line bundle on $S$ corresponding to $\left(\operatorname{det} R f_{*} \mathscr{E}\right)^{-1}$. We note that there exists a canonical isomorphism

$$
\lambda(E)_{s} \cong \lambda\left(E_{s}\right),
$$

where $\lambda\left(E_{s}\right)$ is defined by $(10.1)$.

On the other hand, $\tilde{\omega}$ defines a $C^{\infty}$ family $\tilde{g}:=\left\{g_{s}\right\}_{s \in S}$ of Kähler metrics $g_{s}$ on each fiber $X_{s}$. Let $k_{s}$ be the Quillen metric on $\lambda\left(E_{s}\right)$ associated to the pair $\left(\left(X_{s}, g_{s}\right),\left(E_{s}, h_{s}\right)\right)$, where $\left(E_{s}, h_{s}\right)$ is the restriction of $(E, h)$ to $X_{s}$. Via the natural isomorphism (10.3) we consider $k=\left\{k_{s}\right\}_{s \in S}$ as a hermitian metric on $\lambda(E)$ and call it the Quillen metric on $\lambda(E)$ associated to the pair $Q=((\mathscr{X}, \tilde{g}),(E, h))$.

Now the chern character ch $E$ of $E$ is written as a certain universal formal power series in the chern classes $c_{q}(E)$ of $E$, while for a hermitian vector bundle $(E, h)$ its chern classes are canonically represented by $d$-closed $C^{\infty}$ forms $c_{q}(E, h)$ of type $(q, q)$, constructed universally from the curvature form of $(E, h)$ (cf. $[\mathrm{K}-\mathrm{N}]$ ). (This is true also on any complex space as the arguments of $[\mathrm{K}-\mathrm{N}]$ shows.) By substituting these in the formula for ch $E$ we get a $C^{\infty} d$-closed differential form $\operatorname{ch}(E, h)$ on $X$; for example if $E$ is a line bundle $\mathscr{L}$, we have 


$$
\operatorname{ch}(\mathscr{L}, h)=1+c_{1}(\mathscr{L}, h)+c_{1}(\mathscr{L}, h)^{2} / 2 !+\cdots .
$$

Similarly, by using the total todd polynomial $\operatorname{td}(\mathscr{X} / S)$ associated to the relative tangent bundle $T_{\mathscr{X} / S}$ we define a $d$-closed $C^{\infty}$ form $\operatorname{td}(\mathscr{X} / S, g)$ on $\mathscr{X}$ :

$$
\operatorname{td}(\mathscr{X} / S, g)=1+c_{1}(\mathscr{X} / S, g) / 2+\text { higher degree terms . }
$$

Here $g$ is the hermitian metric of $T_{\mathscr{X} / S}$ defined by $\tilde{g}$.

Now in $[\mathrm{B}-\mathrm{G}-\mathrm{S}]$ the following is proved:

Theorem BGS. Let the notations and assumptions be as above. Suppose that $S$ is nonsingular. Then the Quillen metric $k_{E}$ on $\lambda(E)$ is smooth. The first chern form $c_{1}\left(\lambda(E), k_{E}\right)$ of the hermitian line bundle $\left(\lambda(E), k_{E}\right)$ is given by

$$
c_{1}\left(\lambda(E), k_{E}\right)=-\left[\int_{\mathscr{X} / S} \operatorname{td}(\mathscr{X} / S, g) \operatorname{ch}(E, h)\right]_{2},
$$

where []$_{2}$ denotes the components of degree 2 .

Under a certain assumption on the fiber integral in (10.5) the result turns out to be true even when $S$ has singularities. Namely, from the above theorem we shall deduce in Section 12 the following:

Theorem 10.1. For any reduced complex space $S$ the conclusion of the above theorem still holds true, provided that the right hand side of $(10.5)$ is locally $\partial \bar{\partial}$-exact on $S$.

Remark 10.1. We call any expression of the form $E=F-G$, where $F$ and $G$ are holomorphic vector bundles on $X$, a virtual vector bundle. Then a chern character is extended to any such $E$ simply by setting $\operatorname{ch}(E)=\operatorname{ch}(F)-\operatorname{ch}(G)$. Correspondingly, if $F$ and $G$ carry hermitian metrics $h_{F}$ and $h_{G}$ respectively, we can also define the chern character form ch $\left(E, h_{E}\right)$ by

$$
\operatorname{ch}\left(E, h_{E}\right)=\operatorname{ch}\left(F, h_{F}\right)-\operatorname{ch}\left(G, h_{G}\right),
$$

where $h_{E}$ is the "virtual" hermitian metric on $E$ determined by $h_{F}$ and $h_{G}$. In this situation we define the determinant bundle $\lambda(E)$ similarly by $\lambda(E)=\lambda(F) \otimes \lambda(G)^{-1}$ with Quillen metric $k_{E}$ defined by $k_{F} \otimes k_{G}^{-1}$. Then under these extended definitions the above theorems still hold true for any virtual hermitian vector bundles, as is immediately seen from the fact that the both sides of $(10.5)$ is additive in $(E, h)$.

Let $(\mathscr{L}, h)$ be a holomorphic hermitian line bundle on $\mathscr{X}$. Let $K_{\mathscr{X} / \boldsymbol{S}}$ be the relative canonical bundle with the hermitian metric induced by $g$. We are interested in the following virtual hermitian bundles:

$$
\begin{gathered}
E=\left(\mathscr{L}-\mathscr{L}^{-1}\right)^{\otimes n} \otimes\left(K_{\mathscr{X} / S}-K_{\mathscr{X} / S}^{-1}\right) \\
F=\mathscr{L} \otimes\left(\mathscr{L}-\mathscr{L}^{-1}\right)^{\otimes n},
\end{gathered}
$$

where $n=\operatorname{dim} X_{s}$ and in general

$$
\left(E_{1}-E_{2}\right) \otimes\left(E_{1}^{\prime}-E_{2}^{\prime}\right)=\left(E_{1} \otimes E_{1}^{\prime}\right) \oplus\left(E_{2} \otimes E_{2}^{\prime}\right)-\left(E_{1} \otimes E_{2}^{\prime}\right) \oplus\left(E_{1}^{\prime} \otimes E_{2}\right) .
$$


By Remark 10.1, for $E$ and $F$ the chern characters and the determinant bundles with Quillen metrics are defined. We get

$$
\operatorname{ch}\left(\left(\mathscr{L}-\mathscr{L}^{-1}\right)^{k+1}\right)=2^{k+1} c_{1}(\mathscr{L}, h)^{k+1}+\cdots
$$

and then

$$
\begin{aligned}
& \operatorname{ch}\left(E, h_{E}\right)=-2^{n+1} c_{1}(\mathscr{L}, h)^{n} c_{1}(\mathscr{X} / S, g)+\cdots \\
& \operatorname{ch}\left(F, h_{F}\right)=2^{n} c_{1}(\mathscr{L}, h)^{n}+2^{n-1} c_{1}(\mathscr{L}, h)^{n+1}+\cdots
\end{aligned}
$$

where $\cdots$ denotes the higher degree terms.

Proposition 10.2. Let $E$ and $F$ be as above. Then the first chern forms $c_{1}\left(\lambda(E), k_{E}\right)$ and $c_{1}\left(\lambda(F), k_{F}\right)$ are given respectively by

and

$$
-2^{n+1} \int_{\mathscr{X} / S} c_{1}(\mathscr{L}, h)^{n} c_{1}(\mathscr{X} / S, g),
$$

$$
2^{n-1}\left(\int_{\mathscr{X} / S} c_{1}(\mathscr{L}, h)^{n} c_{1}(\mathscr{X} / S, g)-2 \int_{\mathscr{X} / S} c_{1}(\mathscr{L}, h)^{n+1}\right) .
$$

Proof. By Varouchas (cf. Proposition 1.3) the above fiber integrals are both locally $\partial \bar{\partial}$-exact on $S$. Hence the result follows from Theorem 10.1, (10.4) and (10.7).

The idea of using virtual vector bundles in a similar situation is due to Donaldson [D].

For a polarized Hodge manifold $(X, L)$ of dimension $n$ we define integers $d(X, L)$ and $e(X, L)$ by $d(X, L)=c_{1}(X) \cdot c_{1}(L)^{n-1}$ and $e(X, L)=c_{1}(L)^{n}$ respectively. Let $(f: X \rightarrow$ $S, \mathscr{L}$ ) be a polarized family of Hodge manifolds with $S$ connected and with fiber dimension $n$. Then we define a virtual vector bundle $E(f, \mathscr{L})$ on $\mathscr{X}$ by

$$
E(f, \mathscr{L})=E^{\oplus(n d-2(n+1) e)}+F^{\oplus 4 n d},
$$

where $d=d\left(X_{s}, L_{s}\right)$ and $e\left(X_{s}, L_{s}\right)$ are independent of $s$. If we have a hermitian metric $h$ on $\mathscr{L}$, we get the Quillen metric $k_{E(f, \mathscr{L})}(h)$ on the corresponding determinant line bundle $\lambda(E(f, \mathscr{L}))$.

Theorem 10.3. Let $(f: \mathscr{X} \rightarrow S, \tilde{\omega}, \mathscr{L})$ be a family of extremal Hodge manifolds with $S$ connected, and $h$ an admissible hermitian metric on $\mathscr{L}$ for $(f, \tilde{\omega})$. Then the first chern form of the hermitian line bundle $(\lambda, k):=\left(\lambda(E(f, \mathscr{L})), k_{E(f, \mathscr{L})}(h)\right)$ is given by

$$
c_{1}(\lambda, k)=A_{n} \hat{\omega}_{W P}, \quad A_{n}=-\left(2^{n+1} e n !\right) / \pi .
$$

Proof. Set $r=b /(n+1)=(n / n+1)(d / e)$, where $b=b(f, \tilde{\omega})$ is as in (7.1). By Proposition 10.2 we get

$$
\begin{aligned}
& 2^{n+2}(n+1) e\left(\int_{\mathscr{X} / S} c_{1}(\mathscr{X} / S, g) c_{1}(\mathscr{L}, h)^{n}-r \int_{\mathscr{X} / S} c_{1}(\mathscr{L}, h)^{n+1}\right) \\
& \quad=(n+1) e\left((r-2) c_{1}\left(\lambda(E), k_{E}\right)+4 r c_{1}\left(\lambda(F), k_{F}\right)\right) \\
& \quad=c_{1}(\lambda, k) .
\end{aligned}
$$


The theorem then follows from Theorem 7.8 applied to $\omega_{\mathscr{X}}=c_{1}(\mathscr{L}, h)$, in view of Lemma 7.1 and Remark 7.1.

Corollary 10.4 . Let $(f: \mathscr{X} \rightarrow S, \tilde{\omega}, \mathscr{L})$ be any family of extremal Hodge manifolds. Then $A_{n} \tilde{\omega}_{W P}$ is a first chern form of some hermitian line bundle on $S$. In particular, $A_{n}\left[\tilde{\omega}_{W P}\right]$ is an integral class.

Proof. By Proposition 3.10 there exists a hermitian metric $h$ on $\mathscr{L}$ which is admissible for $(f, \tilde{\omega})$. The corollary thus follows from Theorem 10.3.

Remark 10.2. If $h$ is normalized in the above theorem (cf. Def. 3.9) we have similarly the following simpler formula:

$$
c_{1}\left(\lambda(E), k_{E}\right)=B_{n} \hat{\omega}_{W P}, \quad c_{1}\left(\lambda(F), k_{F}\right)=-\left(B_{n} / 4\right) \hat{\omega}_{W P}
$$

where $B_{n}=2^{n} n ! / \pi$. But the global existence of such a metric is not guaranteed (cf. Proposition 3.12).

On the other hand, in the $\mathbb{K a ̈ h l e r - E i n s t e i n ~ c a s e ~ t h e r e ~ e x i s t s ~ a ~ s i m i l a r ~ c o n s t r u c t i o n ~ o f ~}$ a hermitian line bundle on $S$ which turns out to globalize naturally to the corresponding moduli space (cf. Sect. 11).

Let $(f: \mathscr{X} \rightarrow S, \tilde{\omega})$ be a family of $\mathbb{K a ̈ h l e r - E i n s t e i n ~ m a n i f o l d s ~ w i t h ~} c_{1}\left(X_{s}\right) \neq 0$ for any $s$. We then consider the following virtual hermitian vector bundle

$$
G=\left(K_{\mathscr{X} / S}-K_{\mathscr{X} / S}^{-1}\right)^{n+1}, \quad n=\operatorname{dim} X_{s},
$$

whose chern form is given by

$$
\operatorname{ch}\left(G, h_{G}\right)=(-1)^{n+1} 2^{n+1} c_{1}(\mathscr{X} / S, g)^{n+1}+\cdots .
$$

Then by Theorem 7.9 we get similarly

Theorem 10.5. Let $(f: \mathscr{X} \rightarrow S, \tilde{\omega})$ be as above. Let $\left(\lambda(G), k_{G}\right)$ be the determinant line bundle with Quillen metric associated to $G$. Then we get

$$
c_{1}\left(\lambda(G), k_{G}\right)=(-1)^{n}\left(2 / r^{n}\right)(n+1) ! / \pi \omega_{W P},
$$

where $\omega_{s} \in r c_{1}\left(X_{s}\right)$.

\section{§1耳. Positive Lime Brumdles on the Mloduli Spaces of Extremal Hodge and

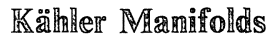

In this section, by globalizing the construction of the previous section we construct a line bundle with a hermitian $V$-metric on the moduli spaces of extremal Hodge and Kähler manifolds whose chern $V$-form coincides with the generalized Weil-Petersson $V$-form up to a constant. A similar but a slightly better result will be obtained for the moduli space of canonically polarized manifolds also. Especially, we shall see that any compact subvariety of these moduli spaces is projective. The method is to compare our construction with the (global) Hilbert scheme construction in algebraic geometry.

We now state the results precisely. Fix a compact connected $\mathbb{C}^{\infty}$ manifold $M$ and 
a class $\alpha \in H^{2}(M, \mathbb{R})$. We assume that $\alpha$ is integral, i.e., $\alpha \in \operatorname{Im}\left(H^{2}(M, \mathbb{Z}) \rightarrow H^{2}(M, \mathbb{R})\right)$. We consider the moduli spaces $\mathfrak{M}_{H, e}$ and $\mathfrak{M}_{e}$ of extremal Hodge and Kähler manifolds respectively with the underlying $C^{\infty}$ structure $(M, \alpha)$.

Theorem 11.1. There exist a holomorphic line bundle $F$ on $\mathfrak{M}_{H, e}$ and a hermitian $V$-metric $k$ on $F$ with respect to the natural complex $V$-structure on $\mathfrak{M}_{H, e}$ such that the first chern $V$-form $c_{1}(F, k)$ coincides with the generalized $W P V$-form on $\mathfrak{M}_{H, e}$ up to positive constants which may depend on the connected components of $\mathfrak{M}_{H, e}$.

See Definition 1.6 for the terminologies. By using this theorem we also obtain:

Theorem 11.2. The same statement as in Theorem 11.1 is true also for $\mathfrak{M}_{e}$.

In particular, the generalized $W P$ classes $\left[\hat{\omega}_{W P}\right]$ and $\left[\omega_{W P}\right]$ (cf. Def. 1.8) are integral classes up to positive constants. By Proposition 1.5 we get:

Corollary 11.3. Any compact analytic subspace $\mathfrak{M}_{H, e}$ and $\mathfrak{M}_{e}$ is projective.

Corollary 11.4 . Let $(f: \mathscr{X} \rightarrow S, \tilde{\omega})$ be a family of extremal Kähler manifolds such that the Kähler classes of $\omega_{s}$ are all integral. Then there exists a hermitian line bundle $\left(F_{S}, k_{S}\right)$ on $S$ whose first chern form coincides with the generalized WP form $\omega_{W P}$ associated to $(f, \tilde{\omega})$ up to a positive constant on each connected component of $S$.

Proof. The natural map $\pi: S \rightarrow \mathfrak{M}_{e}$ is clearly a $V$-morphism in the sense of Definition 1.9. Therefore, the hermitian $V$-metric $\pi^{*} k$ on $\pi^{*} F$ is actually an ordinary metric, where $(F, k)$ is a hermitian line bundle on $\mathfrak{M}_{e}$ in Theorem 11.2. Since the pull-back of the generalized $W P$ form on $\mathfrak{M}_{e}$ is that on $S$, the corollary follows from Theorem 11.2.

We now turn to the proof of Theorem 11.1. We need some preliminary considerations. For any numerical polynomial $P=P(Y) \in \mathbb{Q}[Y]$ let $\mathfrak{M}_{H}^{P}$ be the set of isomorphism classes of polarized Hodge manifolds $(X, L)$ with Hilbert polynomial $P$, i.e., $P(m)=\chi\left(X, L^{m}\right):=\Sigma_{q \geqq 0}(-1)^{q} \operatorname{dim} H^{q}\left(X, L^{m}\right)$ for every $m$. Each $\mathfrak{M}_{H}^{P}$ is open and closed in $\mathfrak{M}_{H}$, and $\mathfrak{M}_{H}$ is a disjoint union $\mathfrak{M}_{H}=\coprod_{P} \mathfrak{M}_{H}^{P}$. We set $\mathfrak{M}_{H, e}^{P}:=\lambda^{-1}\left(\mathfrak{M}_{H}^{P}\right)$. Then $\mathfrak{M}_{H, e}$ also is decomposed into a similar disjoint union $\mathfrak{M}_{H, e}=\coprod_{P} \mathfrak{M}_{H, e}^{P}$.

We shall first recall the algebraic construction of $\mathfrak{M}_{H}^{P}$ mentioned in Remark 5.3, which is based on Matsusaka's big theorem [Ma]. Namely, the latter implies the following lemma (cf. $[\mathrm{P}]$ ). We denote by $\mathbb{P}^{N}$ the complex projective space, by Hilb $\mathbb{P}^{N}$ the Hilbert scheme of $\mathbb{P}^{N}$ and by $H$ the hyperplane bundle on $\mathbb{P}^{N}$.

Lemma 11.5. There exist positive integers $N$ and $m$, and a Zariski open subset $U$ of $\left(\mathrm{Hilb}_{\mathbf{P}^{N}}\right)_{\mathrm{red}}$ with the following properties: For any $\left.u \in U: 1\right)$ The corresponding subspace $X_{u}$ of $\mathbb{P}^{N}$ is smooth. 2) There exists a positive line bundle $L_{u}$ on $X_{u}$ such that the isomorphism class of $\left(X_{u}, L_{u}\right)$ belongs to $\mathfrak{M}_{H}^{P}$ and $\left(X_{u}, L_{u}^{m}\right) \cong\left(X_{u}, H_{u}\right)$, where $H_{u}$ is the restriction of $H$ to $X_{u}$. 3) Conversely, any point of $\mathfrak{M}_{H}^{P}$ is represented by some $\left(X_{u}, L_{u}\right)$ as above. 4) The restriction map $\Gamma\left(\mathbb{P}^{N}, H\right) \rightarrow \Gamma\left(X_{u}, H_{u}\right)$ is isomorphic for any $u$. Finally, 5) $U$ is invariant under the action of $G:=P G L(N+1, \mathbb{C})$ on $\mathrm{Hilb}_{\mathrm{P}^{N}}$ and the action is with finite stabilizer $\partial t u$ where $X_{u}$ is in $\mathscr{A}$. 
Let $V=\left\{\left(u, L_{u}\right) ; u \in U, \mathbb{L}_{u} \in \mathbb{P i c} X_{u}, \mathbb{L}_{u}^{m} \cong \mathbb{H}\right\}$. Then the natural projection $\beta: \mathbb{V} \rightarrow$ $U$ makes $V$ a finite unramified covering of $U$, the natural action of $G$ on $U$ lifts to a proper action on $V$, and by construction we have a natural isomorphism $\mathfrak{D P}_{H}^{P} \underset{\rightarrow}{\rightarrow} / G$ of complex spaces.

Let $p_{0}: Z_{0} \rightarrow \mathbb{V}$ be the pull-back of the universal family over $\mathbb{H i l b}_{\mathbb{P}^{N}}$ by the natural map $\beta: V \rightarrow U \subseteq \mathbb{H i l b}_{\mathbb{P}^{N}}$, and $N_{0} \rightarrow Z_{0}$ the pull-back of $H^{N+1} \rightarrow \mathbb{P}^{N}$ via the projection $Z_{0} \rightarrow \mathbb{P}^{N}$. We get the polarized family $\left(p_{0}, N_{0}\right)$ of Hodge manifolds, to which the $G$-action on $\mathbb{V}$ naturally lifts. We further set

$W=\left\{\left(v, \omega_{v}\right) ; v \in V, \omega_{v}\right.$ is an extremal $\mathbb{K a ̈ h l e r}$ form on $X_{u}$ with $\left.\omega_{v} \in c_{1}\left(N_{0 v}\right)\right\}$.

Then by Theorem 6.3 we can put a natural structure of a complex space on $W$ such that the projection $\delta: W \rightarrow V$ is locally biholomorphic. Moreover, by the definition of $W$ the pull-back $(p: \mathbb{Z} \rightarrow W, N)$ of $\left(p_{0}, N_{0}\right)$ is turned naturally into a family $(p: \mathbb{Z} \rightarrow$ $W, \tilde{\omega}, N)$ of extremal Hodge manifolds. The natural $G$-action on $\left(p_{0}, N_{0}\right)$ lifts further to a natural action on the family $(p, \tilde{\omega}, N)$. Then by the construction we get a natural isomorphism of complex spaces

$$
\mathfrak{D N}_{H, e}^{P} \stackrel{\sim}{\rightarrow} \mathbb{W} / G .
$$

Lemma 11.6. There exists a G-invariant hermitian metric $h$ on $N$ which is admissible for $(p, \tilde{\omega})$.

Proof. Let $\alpha: W \rightarrow \bar{W}:=W / G$ be the natural projection. For any point $\bar{w} \in \bar{W}$ take a point $w \in W$ with $\alpha(w)=\bar{w}$. Take a local slice for the action of $G$ on $W$, i.e., a locally closed $G_{w}$-invariant analytic subset $\mathbb{E}$ of $\mathbb{W}$ passing through $w$ such that the natural map $E / G_{w} \rightarrow \bar{E}:=\alpha(E) \subseteq \bar{W}$ is isomorphic, where $G_{w}$ is the stabilizer at $w$ (cf. [H]). Take any $G_{w}$-invariant hermitian metric $h_{E}$ on $N \mid Z_{E}$, which is admissible for the restricted family $\left(Z_{E} \rightarrow E, \omega_{E}\right)$ over $E$ (cf. Proposition 3.10). Then define a $G$-invariant $C^{\infty}$ hermitian metric $h_{\bar{E}}$ on $N$ over $\alpha^{-1}(\bar{E})$, which is admissible for $(p, \tilde{\omega})$ by the condition; $h_{E, z}=g^{*} h_{E, g^{-1}(z)}$, where $g$ is any element of $G$ with $g^{-1}(z) \in \mathbb{Z}_{E}$. It is then standard to see that the definition is independent of the choice of such a $g$ and gives a $G$-invariant $\mathbb{C}^{\infty}$ hermitian metric. The admissibility is clear by the definition.

Now cover $\bar{W}$ by a locally finite open covering $\overline{\mathscr{E}}=\left\{E_{i}\right\}$ such that $\bar{E}_{i}$ is obtained as $\bar{E}$ as above. Let $h_{i}$ be a $G$-invariant hermitian metric on $N$ over $\widetilde{E}_{i}=\alpha^{-1}\left(\bar{E}_{i}\right)$ which is admissible for $(p, \tilde{\omega})$. Then by Proposition 3.10 over $\widetilde{E}_{i} \cap \widetilde{E}_{j}$ we have $h_{i} / h_{j}=p^{*} e^{q_{\tau_{j}}}$ for some $C^{\infty}$ function $q_{i j}$ on $\widetilde{E}_{i} \cap \widetilde{E}_{j}$, which is $G$-invariant by the $G$-invariance of $h_{i}$ and $h_{j}$. Hence, $q_{i j}$ is considered as a section on $\bar{E}_{i} \cap \bar{E}_{j}$ of the G-invariant direct image sheaf $\alpha_{*}^{G} \mathscr{D}_{W}$ on $\bar{W}$. Thus $\left\{q_{i j}\right\}$ defines a 1 -cocycle with respect to the covering $\overline{\mathscr{E}}$ with coefficients in $\alpha_{*}^{G} \mathscr{D}_{W}$. Since $\alpha_{*}^{G} \mathscr{D}_{W}$ is a fine sheaf, $H^{1}\left(\overline{\mathscr{E}}, \alpha_{*}^{G} \mathscr{D}_{W}\right)=0$. Therefore we can find elements $q_{i} \in \Gamma\left(\bar{E}_{i}, \alpha_{*}^{G} \mathscr{D}_{W}\right)$ such that $q_{i j}=q_{i}-q_{j}$. Then if we set $h_{i}^{\prime}=h_{i} p^{*} e^{q_{1}}$, these patch together globally to give a desired hermitian metric on $N$.

We also note a simple general lemma.

Lemma 11.7. Let $Y$ be a complex space and $F$ a holomorphic line bundle on $\mathbb{Y}$. Let $G$ be a finite group acting on $F \rightarrow \mathbb{Y}$ as bundle automorphisms. Suppose that for any $y \in Y$ the stabilizer $G_{y}$ at $y$ acts trivially on the fiber $\mathbb{F}_{y}$. Then the induced map 
$F / G \rightarrow Y / G$ makes $F / G$ again a line bundle over the quotient analytic space $Y / G$ such that $\pi^{*}(F / G) \cong \mathbb{F}$ canonically, where $\pi: Y \rightarrow Y / G$ is the projection.

Proof. Let $y$ be any point of $Y$. Take a local trivialization $F \mid V \cong V \times \mathbb{C}$ on some $G_{y}$-invariant neighborhood $V$ of $y$ such that $g V \cap V=\phi$ for any $g \notin G_{y}$. Then any element $g$ of $G_{y}$ acts on $V \times \mathbb{C}$ in the form $f(v, \zeta)=(g(v), \zeta),(v, \zeta) \in V \times \mathbb{C}$. Hence, in a neighborhood of the image of $y$ in $Y / G, F / G \rightarrow Y / G$ is isomorphic to the projection $(V / G) \times \mathbb{C} \rightarrow V / G$. It follows that $F / G$ is a line bundle on $Y / G$ with the desired property.

Proof of Theorem 11.1. We need only to prove the statement for each $\mathfrak{M}_{H, e}^{P}$. By Lemma 11.6 there exists a $G$-invariant hermitian metric $h$ on $N$ which is admissible for $(p, \tilde{\omega})$. Take any extremal Hodge manifold $(X, \omega, L)$ in $\mathfrak{M}_{H, e}^{P}$. In the notations of Section 6 let $S=S(X, \omega, L)$ and $H=G(X, \omega, L)$. Let $\mathscr{F}=(\pi: S \rightarrow \bar{S}, H)$ be the associated local Galois cover of $\mathfrak{M P}_{H, e}^{P}$. Take and fix any point $w \in W$ such that $\left(Z_{w}, \omega_{w}, N_{w}\right)$ is isomorphic to $(X, \omega, L)$. Then by a standard argument we can find a local $G_{w^{-}}$ invariant slice $E$ at $w$ for the $G$-action and an isomorphism of the induced family $\left(p_{E}: Z_{E} \rightarrow E, \tilde{\omega}_{E}, N_{E}\right)$ and the universal family $\left(f: \mathscr{X} \rightarrow S, b \tilde{\omega}, \mathscr{L}^{b}\right)$ over $S$, where $b=$ $m(N+1)$. Moreover, there exists a natural isomorphism $\mu_{w}: G_{w} \neg H$ such that the isomorphism $E \rightarrow S$ is $\mu_{w}$-equivariant. We consider the induced hermitian metric $h_{S}$ on $\mathscr{L}^{b}$ from $h \mid Z_{E}$ by the above isomorphism. Similarly to $G_{w}$, the action of $H$ on $S$ then lifts canonically to an action on the family $\left(f, b \tilde{\omega}, \mathscr{L}^{b}\right)$ preserving $h_{S}$.

We take the determinant bundle $\lambda_{s}:=\lambda\left(E\left(f, \mathscr{L}^{b}\right)\right)$ associated to the virtual vector bundle $E\left(f, \mathscr{L}^{b}\right)$ (cf. (10.8)). Let $k_{S}=k_{E\left(f, \mathscr{L}^{b}\right)}\left(h_{S}\right)$ be the corresponding Quillen metric on $\lambda_{S}$. The action of $H$ on $S$ lifts naturally to the action on $\left(\lambda_{S}, k_{S}\right)$, and hence on $\left(\lambda_{S}^{c}, k_{S}^{c}\right)$ for any integer $c$. By Theorem 10.3 we get $c_{1}\left(\lambda_{S}, k_{S}\right)=A_{n} b^{n} \hat{\omega}_{W P}$, where $\hat{\omega}_{W P}$ is the generalized $W P$ form for the family $(f, \tilde{\omega}, \mathscr{L})$.

On the other hand, the action of $G$ on $U$ is algebraic with $U$ quasi-projective; so the set of the orders of the stabilizers of this action is finite. Hence the same is true for the action of $G$ on $W$ because $G_{w} \subseteq G_{\beta \delta(w)}$. Take $c$ to be a common multiple of the orders of $G_{w}$ for all $w \in W$. Then by Lemma $11.7 \lambda_{S}^{c}$ descends to a line bundle, say $F_{S}$ on $S / G \subseteq \mathfrak{D P}_{H, e}^{P}$.

We show that $\left\{\lambda_{S}\right\}$ defines a line $V$-bundle on $\mathfrak{M}_{H, e}$ and $\left\{k_{S}\right\}$ defines a hermitian $V$-metric on this $V$-bundle (cf. the remark before Definition 1.9). By what we have seen above and Lemma 11.7 this would then imply that $\lambda_{S}^{c}$ descends to a line bundle, say $F$, on $\mathfrak{M}_{H, e}^{P}$ and $\left\{k_{S}^{b}\right\}$ defines a hermitian $V$-metric on $F$. It is clear that the pair $\left(F,\left\{k_{S}^{b}\right\}\right)$ satisfies the condition of the theorem.

Let $\left(X^{\prime}, \omega^{\prime}, L^{\prime}\right)$ be another Hodge manifold and write $S^{\prime}=S\left(X^{\prime}, \omega^{\prime}, L^{\prime}\right)$ and $H^{\prime}=$ $G\left(X^{\prime}, \omega^{\prime}, \mathbb{L}^{\prime}\right)$. Assume that $S^{\prime} / H^{\prime} \subseteq S / H \subseteq \mathfrak{D P}_{H, e}^{P}$ and that $\mathscr{L}^{\prime b}$ is given a hermitian metric $h_{S^{\prime}}$ via an isomorphism of $\left(f^{\prime}, b \tilde{\omega}^{\prime}, \mathscr{L}^{\prime b}\right)$ with $\left(p_{E^{\prime}}: Z_{E^{\prime}} \rightarrow E^{\prime}, \tilde{\omega}_{E^{\prime}}, N_{E^{\prime}}\right)$, where $E^{\prime}$ is a fixed local slice at a point $w^{\prime} \in \alpha^{-1}(\alpha(E))$. It suffices to show that there exist an inclusion $l: H^{\prime} \rightarrow H$, an $l$-equivariant morphism $\tau: S^{\prime} \rightarrow S$, and an isomorphism of the universal family $\left(f^{\prime}, b \tilde{\omega}^{\prime}, \mathscr{L}^{\prime b}\right)$ and the pull-back of $\left(f, b \tilde{\omega}, \mathscr{L}^{b}\right)$ by $\tau$ sending $h_{S^{\prime}}$ to $h_{S}$. This however follows readily from the fact that there exists a morphism $v: E^{\prime} \rightarrow G$ such that $\mu(e):=v(e)(e) \in E$ and $\mu\left(w^{\prime}\right)=w$; then the map $\mu: E^{\prime} \rightarrow E$ clearly lifts to a map of the corresponding families $\left(p_{E}, \tilde{\omega}_{E}, N_{E}\right)$ and $\left(p_{E^{\prime}}, \tilde{\omega}_{E^{\prime}}, N_{E^{\prime}}\right)$ sending the hermitian metrics $h_{E^{\prime}}$ to $h_{E}, h$ being $G$-invariant. 
Proof of Theorem 11.2. Let $(X, \omega)$ be any extremal compact $\mathbb{K} a ̈ h l e r$ manifold in $\mathfrak{M}_{e}$. We recall the commutative diagram $(9.4)$ associated to $(X, \omega)$, and especially the $V$-morphism $a: P \rightarrow \mathfrak{M}_{H, e}$. We set $\hat{F}=a^{*} F$ and $\hat{k}=a^{*} k$, where $(F, k)$ is a $(V$-) hermitian line bundle satisfying the condition of Theorem 11.1 on $\mathfrak{D l}_{H, e}$. Then $(\hat{F}, \hat{k})$ is a hermitian line bundle in the usual sense on $P$ and $c_{1}(\hat{F}, \hat{k})=c \bar{\omega}_{W P}$, where $c$ is a positive constant depending only on connected components of $\mathfrak{M}_{e}$ containing $(X, \omega)$, and $\bar{\omega}_{W P}=$ $a^{*} \hat{\omega}_{W P}\left(\mathfrak{M}_{H, e}\right)$. We take the determinant bundle with Quillen metric $\left(\bar{\lambda}_{S}, \bar{k}_{s}\right)$ associated to the virtual hermitian vector bundle $\left(\hat{F}-\hat{F}^{-1}\right)^{m+1}$ on $P$, where $m$ is the dimension of the fibers of $P \rightarrow S$. Here, $\left(\bar{\lambda}_{S}, \bar{k}_{S}\right)$ admits a natural $G$-action since $(\hat{F}, \hat{k})$ is $G$-invariant, where $G=G(X, \omega)$.

On the other hand, by combining (10.6) and Theorem 11.1, as in Proposition 10.2 we get

$$
c_{1}(\bar{\lambda}, \bar{k})=-2^{m+1} \int_{P / S} c_{1}(\hat{F}, \hat{k})^{m+1}=-2^{m+1} \tilde{c} \int_{P / S} \bar{\omega}_{W P}^{m+1}, \quad \tilde{c}=c^{m+1} .
$$

By Proposition 9.5 the last term equals $-2^{m+1} \tilde{c} C(m+1) \omega_{W P}^{m+1}$, where $C$ is a positive constant depending only on the connected component of $\mathfrak{M P}_{e}$ containing $(X, \omega)$.

We claim that $\bar{\lambda}:=\left\{\bar{\lambda}_{s}\right\}$ determines a line $V$-bundle on $\mathfrak{D e}_{e}$ and $\bar{k}:=\left\{\bar{k}_{s}\right\}$ the hermitian $V$-metric on it. Let $\left(X^{\prime}, \omega^{\prime}\right)$ be another extremal compact $\mathbb{K} a ̈ h l e r$ manifold in $\mathfrak{M}_{e}$ such that $S^{\prime} / G^{\prime} \subseteq S / G \subseteq \mathbb{D}_{e}$, where $S^{\prime}=S\left(X^{\prime}, \omega^{\prime}\right)$ and $G^{\prime}=G\left(X^{\prime}, \omega^{\prime}\right)$. Let $\pi^{\prime}: \mathbb{P}^{\prime} \rightarrow$ $S^{\prime}$ and $a^{\prime}: P^{\prime} \rightarrow M$ be the corresponding morphisms for $\left(X^{\prime}, \omega^{\prime}\right)$. Then for any open embedding $\check{j}: S^{\prime} \rightarrow S$ over $S^{\prime} / G^{\prime} \subseteq S / G$ which lifts to a morphism of corresponding universal families lifts also to a morphism $j_{P}: \mathbb{P}^{\prime} \rightarrow \mathbb{P}$ with $a^{\prime}=a j_{P}$. From this and the construction the assertion follows immediately.

Finally, by taking a suitable power of $(\bar{\lambda}, \bar{k})$ on each connected component we get a line bundle on $\mathfrak{M}_{e}$ instead of a $V$-bundle in view of the following lemma.

Lemm2 11.8. On each connected component the orders of the groups $G(\mathbb{X}, \omega)$ are bounded.

Proof. Let $\mathfrak{M}_{e}^{P}$ be the image of $\mathfrak{M}_{H, e}^{P} . \mathfrak{M R}_{e}^{P}$ is open and closed in $\mathfrak{M l}_{e}$. So it suffices to prove that the orders of $G(X,[\omega])$ are bounded on $\mathfrak{M}_{e}^{P}$, where $[\omega]$ denotes the

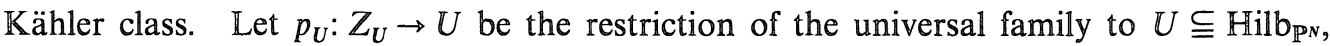
and $\underline{Z}_{U}=\left(p_{U}, \tilde{\gamma}\right)$ the polarized family of $\mathbb{K a ̈ h l e r}$ manifolds with $\gamma_{u}=c_{1}\left(H_{u}\right), u \in \mathbb{U}$. Then $\operatorname{Aut}_{U} \underline{Z}_{U}:=\mathbb{I s o m}_{U}\left(\underline{Z}_{U}, \underline{Z}_{U}\right)$ is naturally a Zariski open subset of an analytic space which is proper over the closure of $U$ in $\mathbb{H i l b}_{\mathbb{P}^{N}}$ (cf. [Fu3]). From this one sees readily that the number of connected components of each fiber of $\mathrm{Aut}_{U} Z_{U}$ is bounded. This implies the desired assertion.

Fix again a numerical polynomial $P=P(Y) \in \mathbb{Q}[Y]$. Next, by using Theorem 10.5 we shall construct a natural positive $V$-line bundle on the (reduced) coarse moduli space $\Omega^{P}$ of canonically polarized projective manifolds with Hilbert polynomial $P$. As a set $\mathfrak{R}^{P}$ is the set of isomorphism classes of projective manifolds $X$ with ample canonical bundle $K_{X}$ such that $P(m)=\chi\left(X, K_{X}^{m}\right)$ for all $m$. Let $U \subseteq\left(\mathrm{Hilb}_{\mathbb{P}^{N}}\right)_{\text {red }}$ be as in Lemma 11.5. Let $U_{1}$ be the closed quasi-projective subspace of $U$ defined by

$$
U_{1}=\left\{u \in \mathbb{U} ; K_{X_{u}}^{m} \cong H_{u}\right\}
$$


Then $U_{1}$ is preserved by $G$ and we have

$$
\mathfrak{R}^{P} \cong U_{1} / G
$$

$\Omega^{P}$ has thus the natural structure of an algebraic space of finite type over $\mathbb{C}$ as the action is proper. Each connected component of $\mathfrak{R}^{P}$ is naturally identified with an open and closed subset of one of the moduli spaces $\mathfrak{M}=\mathfrak{M}_{\alpha}$ in Theorem 5.4; further by the uniqueness of an Einstein-Kähler metric $\kappa: \kappa^{-1}\left(\mathfrak{\Re}^{P} \cap \mathfrak{M}\right) \rightarrow \mathfrak{K}^{P} \cap \mathfrak{M}$ is biholomorphic so that it can also be thought of as an open and closed subset of $\mathfrak{M}_{e}$ in Theorem 6.6. Hence by Theorem 7.10 we get:

Proposition 11.9. $\mathfrak{R}^{P}$ carries a natural complex $V$-structure as a complex space and a natural Kähler V-metric with respect to this $V$-structure.

We call the above Kähler $V$-metric the Weil-Petersson $V$-metric on $\Omega^{P}$. Our purpose is to show that there is a natural algebraic line bundle $\boldsymbol{\Omega}^{P}$, i.e. a line bundle in the category of algebraic spaces, with a hermitian $V$-metric whose first chern $V$-form coincides up to a constant the $W P$ Kähler $V$-form $\omega_{W P}$ on $\mathfrak{R}^{P}$. Namely, we show the following:

Theorem 1 1.10. There exist a natural algebraic line bundle $F$ on $\mathfrak{A}^{P}$ and a natural hermitian $V$-metric $k$ on $F$ such that the first chern $V$-form $c_{1}(F, k)$ coincides with the Weil-Petersson $V$-form on $\mathfrak{\Omega}^{P}$ up to a constant; $c_{1}(F, k)=a_{n} \omega_{W P}$, where $a_{n}=$ $(2 / m)^{n}(n+1) ! / \pi$ for some positive integer $m$, and $n$ is the dimension of the manifolds.

Corollary 11.11. Any compact analytic subspace of $\mathfrak{R}^{P}$ is projective.

The idea is to globalize the hermitian line bundle $\left(\lambda(G), k_{G}\right)$ in Theorem 10.5 to $\Re^{P}$. We first note the strong invariance property of these bundles. Let $f: \mathscr{X} \rightarrow S$ be a family of canonically polarized manifolds in general. Then by the uniqueness of the Kähler-Einstein metric mentioned above there exists a unique metric polarization $\tilde{\omega}$ on $f$ such that $\omega_{s}$ is a Kähler-Einstein form belonging to $-c_{1}\left(X_{s}\right)$ for any $s$. We may then consider the determinant bundle $\lambda(G)$ and the Quillen metric $k_{G}$ as in Theorem 10.5. From the above uniqueness together with the definition of $\left(\lambda(G), k_{G}\right)$ we get:

Lemma 11.12. Let $f_{i}: \mathscr{X}_{i} \rightarrow S_{i}, i=1,2$, be families of canonically polarized manifolds. Then any isomorphism of these two families, i.e., isomorphisms $\tilde{u}: \mathscr{X}_{1} \rightarrow \mathscr{X}_{2}$ and $u: S_{1} \rightarrow S_{2}$ of complex spaces with $f_{2} \tilde{u}=u f_{1}$, induces canonically an isomorphism $\lambda(u)$ : $\left(\lambda(G), k_{G}\right)_{1} \stackrel{\sim}{\rightarrow}\left(\lambda(G), k_{G}\right)_{2}$.

Proof of Theorem 11.10. Take any point of $\mathfrak{A}^{P}$ represented by a canonically polarized manifold $X$. Let $f: \mathscr{X} \rightarrow S$ be a Kuranishi family of $X$. We then have a natural morphism $\pi: S \rightarrow \Re^{P}$ and $H:=$ Aut $X$ acts naturally on $S$ inducing an isomor-

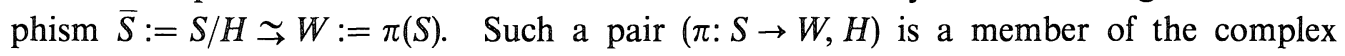
$V$-structure of $\AA^{P}$ and any member is obtained in this way. Let now $(\lambda, k):=\left(\lambda(G), k_{G}\right)$ be the determinant bundle with Quillen metric associated to $f$. Since the action of $H$ on $S$ is induced from its action on $\mathscr{X} \rightarrow S$, by Lemma 11.12 it lifts canonically to an action on $(\lambda, k)$, and hence also on $\left(\lambda^{m}, k^{m}\right)$ for any $m>0$. 
On the other hand, for any $u \in \mathbb{U}_{1}, G_{u}$ is naturally identified with Aut $X_{u}$. Hence the order of the group Aut $X_{u}$ is bounded for all $X$ in $\mathscr{A}^{P}$. Take $m$ to be the common multiple of these integers. Then for any $s \in S$ the stabilizer $\mathbb{H}_{s}$ acts trivially on the fiber $\lambda_{s}^{m}$. Hence, by Lemma $11.7 \lambda^{m}$ descends to a line bundle $\lambda_{m}:=\lambda^{m} / H$ on $\bar{S}$ with $\pi^{*} \lambda_{m} \cong \lambda^{m}$. As in the proof of Theorem 11.1 we conclude that $\lambda_{m}$ patch together to give a global line bundle over the whole $\mathfrak{R}^{P}$, which we shall denote by $F$. The assertion about the first chern $\mathbb{V}$-form is immediate from Theorem 10.5 .

It remains to show that $F$ is algebraic. Let $p_{1}: Z_{1} \rightarrow U_{1}$ be the restriction of the universal family on $\mathbb{H i l b}_{\mathbb{P}^{N}}$. Applying Theorem 10.5 to $p_{1}$ we obtain a determinant bundle with Quillen metric on $U_{1}$, denoted by $\left({ }^{\prime} \lambda, ' k\right)$, where ' $\lambda$ is naturally an algebraic bundle by the construction of $[\mathbb{K}-\mathbb{M}]$. The natural $G$-action on $\mathbb{Z}_{1} \rightarrow U_{1}$ induces by Lemma 11.12 an algebraic action on ${ }^{\prime} \lambda \rightarrow U_{1}$ and hence also on ' $\lambda^{m}$. The quotient space $\lambda_{m}:={ }^{\prime} \lambda^{m} / G$ has again a natural structure of an algebraic space with a natural algebraic morphism onto $\bar{U}_{1}:=U_{1} / G$.

Now let $u$ be an arbitrary point of $U_{1}$ such that $\bar{u}=\mu(u)$, where $\mu: \mathbb{U}_{1} \rightarrow \bar{U}_{1}$ is the natural projection. The quotient algebraic structure of ' $\lambda^{m}$ is obtained locally at $\bar{u}$ by 1) taking a $G_{u}$-invariant slice $V=V_{u}$ at $u$ which is an affine subscheme of $U_{1}$ and then, 2) identifying $\left(\lambda^{m} \mid \mathbb{V}\right) / G_{u}$ with ' $\lambda_{m} \mid \mu(\mathbb{V})$ on the open subset $\mu(\mathbb{V})$. Since $G_{u}$ acts trivially on the fiber ' $\lambda_{u}^{m}$ for any $u \in \mathbb{U}_{1}$, by Lemma $11.7 \quad \lambda_{m}$ is indeed a line bundle on $\bar{U}_{1}=\mathfrak{A}^{P}$.

We claim that $F$ is naturally isomorphic to ' $\lambda_{m}$ as a holomorphic line bundle, and hence, has a natural algebraic structure.

Let $u \in U_{1}$ and $V$ be as above. Denote by $f: \mathscr{X} \rightarrow S$ a Kuranishi family of $X=Z_{u}$. Let $\mathbb{V}_{S}=V \cap \mu^{-1}(W)$ with the natural projection $\mu_{S}: V_{S} \rightarrow \mathbb{W}$. We have then the universal morphism $\tau: V_{S} \rightarrow S$ associated to the induced family $\mathbb{Z} \times_{U} V_{S} \rightarrow \mathbb{V}_{S}$ which is $\left(G_{u}, \mathbb{H}\right)$ equivariant with respect to the natural identification $H=G_{u}$ such that $\mu_{S}=\pi \tau$. Since

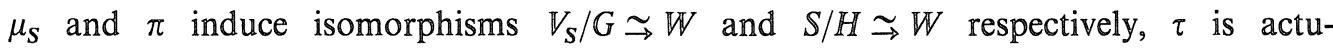
ally biholomorphic. It follows that $\tau$ induces also a $\left(G_{u}, H\right)$-equivariant isomorphism ${ }^{\prime} \lambda^{m} \mid V_{S} \underset{\rightarrow}{\rightarrow} \lambda^{m}$, and hence, we get on $\mathbb{W}$ the canonical isomorphism $\bar{\tau}:{ }^{\prime} \lambda_{m}\left|\mathbb{W} \leadsto \lambda_{m}=\mathbb{F}\right| \mathbb{W}$. By the canonicity of the construction we see that these isomorphisms $\bar{\tau}$ patch together to give a global isomorphism ' $\lambda_{m}$ and $F$ over the whole $\bar{U}_{1}=\Re^{P}$.

\section{\$12. Determimamt Bumalles and Qunihlem Metries with Simgular Base Spaces - Proof of Theorem 10.1}

In this section we prove Theorem 10.1, a generalization of Theorem BGS for singular parameter spaces. The proof is roughly divided into two parts. First, we follow the construction of $[\mathrm{B}-\mathrm{G}-\mathrm{S} \mathbb{I I I}]$ to get a determinant line bundle $\lambda=\lambda^{\mathrm{BGS}}$ and a Quillen metric $k$ on $S$. The point is to show that $(\lambda, k)$ has a natural $\mathbb{C}^{\infty}$ structure which extends the $C^{\infty}$ structure on the smooth part defined in [B-G-S]. This is essentially a consequence of the observation that locally at any point of $S$ our holomorphic families $\left\{\left(X_{s}, g_{s}\right)\right\}$ and $\left\{\left(E_{s}, h_{s}\right)\right\}$ extend to $C^{\infty}$ families of almost hermitian manifolds and of almost hermitian vector bundles respectively over an ambient manifold of $S$.

Secondly, we have to exhibit a natural $C^{\infty}$ isomorphism between $\lambda$ and our (holomorphic) determinant bundle $\lambda^{K M}=\lambda(E)$. Our idea for this is to use the theory of 
relative analytic spaces of Forster-Knorr $[\mathbb{F}-\mathbb{K}]$ (cf. also Schneider $[$ Sch $]$ ) to define a $C^{\infty}$ version $\lambda_{\infty}^{\mathrm{KM}}$ of $\lambda^{\mathrm{KM}}$ to get and use an intermediate of the above two bundles.

We start with explaining the smooth dependence of the analytic torsion on the parameter, which is necessary to see the smoothness of $k$, in a somewhat general setting.

Let $M$ be a compact $C^{\infty}$ manifold and $E$ a $C^{\infty}$ complex vector bundle on $M$. Let $D$ be a domain in some $\mathbb{R}^{k}$. Suppose that we are given a $C^{\infty}$ family $\left\{g_{y}\right\}_{y \in D}$ of $C^{\infty}$ Riemannian metrics on $M$ and a $C^{\infty}$ family $\left\{h_{y}\right\}$ of hermitian metrics $h_{y}$ on $E$. Let $D_{y}: \Gamma\left(E_{y}\right) \rightarrow \Gamma\left(E_{y}\right), y \in D$, be a $C^{\infty}$ family of self-adjoint strongly elliptic operators with respect to these metrics.

Let $b$ be a positive real number which is not an eigenvalue of any of $D_{y}$. Let $K_{y}^{b}$ be the direct sum of the eigenspaces of $D_{y}$ with eigenvalues $\lambda<b$, and $P_{y}^{b}: \Gamma\left(E_{y}\right) \rightarrow K_{y}^{b}$ the orthogonal projection. Set $Q_{y}^{b}=I-P_{y}^{b}$, where $I$ is the identity. Denote by $C$ a smooth path in the complex plane $\mathbb{C}$ encompassing all the eigenvalues $>b$ of $D_{y}$ for any $y$. Then for any $t$ with $0 \leqq t<\infty$ we define as usual

$$
T_{y}^{b}=e^{-t D_{y}} Q_{y}^{b}:=1 / 2 \pi \int_{C} e^{-t \xi}\left(\xi I-D_{y}\right)^{-1} d \xi
$$

This is a bounded linear operator $\Gamma\left(E_{y}\right) \rightarrow \Gamma\left(E_{y}\right)$ with $C^{\infty}$ kernel $p_{y}^{b}\left(t, x, x^{\prime}\right)$; namely we have for any $f \in \Gamma\left(E_{y}\right)$

$$
T_{y}^{b}(f)(x)=\int_{M} p_{y}^{b}\left(t, x, x^{\prime}\right) f\left(x^{\prime}\right) d v_{y}\left(x^{\prime}\right)
$$

where $d v_{y}$ is the volume form associated to $g_{y}$. In fact, by the proof of Proposition 2.8 of $[\mathrm{Bi}] p_{y}^{b}\left(t, x, x^{\prime}\right)$ is of class $C^{\infty}$ in $\left(y, t, x, x^{\prime}\right) \in D \times \mathbb{R}^{+} \times M \times M$.

For any fixed $(y, t), p_{y}^{b}(t, x, x)$ is a $C^{\infty}$ section of End $E_{y}$, whose trace we shall denote by $\operatorname{tr} p_{y}^{b}(t, x, x) \in C^{\infty}(M)$. Then according to [B-G-S III; Def. 1.4] we define

$$
\zeta_{y}^{b}(s)=\int_{0}^{\infty} t^{s-1} \operatorname{Tr}\left(T_{y}^{b}\right) d t, \quad s \in \mathbb{C},
$$

where

$$
\operatorname{Tr} T_{y}^{b}=\int_{M} \operatorname{tr} p_{y}^{b}(t, x, x) d v_{y}(x) .
$$

Then it is known that $\zeta_{y}^{b}(s)$ is holomorphic in $s$ for $\operatorname{Re}(s) \gg 0$, admits a meromorphic continuation to the whole $\mathbb{C}$, and is holomorphic at the origin $o$. In particular, its derivative $\zeta_{y}^{b^{\prime}}(o)$ at $o$ makes sense. The proof of the following proposition is essentially the same as in $[\mathrm{B}-\mathrm{F}]$ (cf. p. 168).

Proposition 12.1. $\zeta_{y}^{b \prime}(o)$ is a smooth function of $y \in D$.

Proof. One reduces the proof easily to showing that $\zeta_{y}^{b}(s)$ is of class $C^{\infty}$ on $D \times U$, where $U$ is some open subset of $\mathbb{C}$ on which $\zeta_{y}^{b}(s)$ are all holomorphic. First of all, $\operatorname{Tr} T_{y}^{b}$ is $C^{\infty}$ on $D \times \mathbb{R}^{+} \times M$ by the above mentioned result of Bismut. Hence, in order to show that $\zeta_{y}^{b}(s)$ is of class $C^{1}$, say, we need only to see that for any coordinate 
function $y$ on $D$ the integral

$$
\int_{0}^{\infty} t^{s-1}\left(\int_{M} \operatorname{tr} \frac{\partial}{\partial y} p_{y}^{b}(t, x, x) d v_{x}\right) d t
$$

converges in locally uniform way with respect to $(y, s) \in D \times \mathbb{U}$. If we take $\mathbb{U}$ so that $\operatorname{Re}(s)$ is sufficiently large for $s \in U$ this is true for $t \rightarrow 0$, i.e., for the integral over $(0,1]$ because of the asymptotic expantion formula which holds for any strongly elliptic operator (cf. [B-F; Th. 1.5]). For $t \rightarrow \infty$ this follows from:

Lemma 12.2. For all $y_{o} \in D$ there exist a neighborhood $U\left(y_{o}\right)$ and constants $0<A$, $0<C<1,0<t_{o}$ such that

$$
\left|\frac{\partial}{\partial y} \int_{M} \operatorname{tr} p_{y}^{b}(t, x, x) d v_{x}\right| \leqq A t C^{t-1} \quad \text { for } t \geqq t_{o}, \quad y \in \mathbb{U}\left(y_{o}\right) .
$$

Proof. First, from the definition of $\mathbb{T}_{y}^{b}$, we get that $p_{y}^{b}\left(t+t^{\prime}, x, x^{\prime}\right)$ is the convolution $\int_{M} p_{y}^{b}\left(t, x, x^{\prime \prime}\right) p_{y}^{b}\left(t^{\prime}, x^{\prime \prime}, x^{\prime}\right) d v_{x^{\prime \prime}}$. Then we use the $n$-fold convolution for $n t, n \in \mathbb{N}$, compute the partial derivative with respect to $y$ and use the inequality

$$
\begin{aligned}
& \left|\int_{M^{n}} f_{1}\left(x_{1}, x_{2}\right) f_{2}\left(x_{2}, x_{3}\right) \ldots f_{n}\left(x_{n}, x_{1}\right) d v_{x_{1}} \ldots d v_{x_{n}}\right| \\
& \quad \leqq\left(\int_{M^{2}} f_{1}^{2}\left(x, x^{\prime}\right) d v_{x} d v_{x^{\prime}} \ldots \int_{M^{2}} f_{n}\left(x, x^{\prime}\right) d v_{x} d v_{x^{\prime}}\right)^{1 / 2} .
\end{aligned}
$$

Now

$$
\int_{M^{2}} \operatorname{Tr}\left(p_{y}^{b}\left(t, x, x^{\prime}\right) p_{y}^{b}\left(t, x^{\prime}, x\right)\right) d v_{x} d v_{x^{\prime}}=\int_{M} \operatorname{tr} p_{y}^{b}(2 t, x, x) d v_{x}<1
$$

for $t \geqq t_{o}$ and $y \in U\left(y_{o}\right)$. From this and a continuity argument we get the claim. (Here $M^{k}$ is the product of $k$-copies of $M$.)

Now we work in the situation of Theorem 10.1. We shall recall the construction of a hermitian line bundle $(\lambda, k)$ from $[\mathrm{B}-\mathrm{G}-\mathrm{S}]$ and show that its $C^{\infty}$ structure on $S_{\text {reg }}$ extends naturally to the whole $S$ (cf. Proposition 12.3 below.)

Let $(f: \mathscr{X} \rightarrow S, \tilde{\omega})$ be a metrically polarized family of compact Kähler manifolds and denote by $g_{s}$ the Kähler metric on $X_{s}$ corresponding to $\omega_{s}$. Let $(E, h)$ be a hermitian vector bundle on $\mathscr{X}$. Denote by $\left(E_{s}, h_{s}\right)$ the restriction of $(E, h)$ to each fiber $X_{s}$. Let $D^{q}\left(E_{s}\right)$ be the space of $E_{s}$-valued $(0, q)$-forms on $X_{s}$ and $\bar{\partial}_{s}^{q}: D^{q}\left(E_{s}\right) \rightarrow D^{q+1}\left(E_{s}\right)$ the usual $\bar{\partial}$-operator on $X_{s}$ with the formal adjoint $\left(\bar{\partial}_{s}^{q}\right)^{*}$ with respect to the metrics $g_{s}$ and $h_{s}$. Then $\square_{s}^{q}:=\left(\bar{\partial}_{s}^{q+1}\right)^{*} \bar{\partial}_{s}^{q}+\bar{\partial}^{q+1}\left(\bar{\partial}^{q}\right)^{*}$ is a strongly elliptic self-adjoint operator. All these operators depend smoothly on $s$.

Now we take a real number $b>0$ and define $U^{b}$ by the open subset of $S$ such that $b$ is not an eigenvalue of $\square_{s}^{q}$ for any $q>0$ and $s \in U^{b}$. Denote by $K_{s}^{b, q}$ the direct sum of all the eigenspaces of $\square_{s}^{q}$ with eigenvalues $<b$. Then we set

$$
\lambda_{s}^{b}=\bigotimes_{q \geqq 0}\left(\lambda_{s}^{b, q}\right)^{(-1)^{q+1}},
$$


where $\lambda_{s}^{b, q}=\bigwedge^{\max } K_{s}^{b, q}$. Then $\lambda^{b}:=\bigcup_{s \in S} \lambda_{s}^{b}$ is a complex line bundle over $U^{b}$ which may a priori not be of class $C^{\infty}$. However, the above construction shows clearly that the restriction of $\lambda^{b}$ to be smooth part $U_{r e g}^{b}$ of $U^{b}$ coincides with the original one defined in [B-G-S III; § 1, b)].

First of all, $h_{s}$ and $g_{s}$ define on $K_{s}^{b, q}$ naturally a hermitian inner product, which in turn gives rise to a natural $C^{\infty}$ hermitian metric ${ }^{\prime} k_{s}^{b}$ on $\lambda_{s}^{b}$. Now we define a positive real number $\tau_{s}$ by

$$
\tau_{s}=\exp \left(-1 / 2 \sum_{q \geqq 0} q \zeta_{s}^{q^{\prime}}(0)\right),
$$

where $\zeta_{s}^{q}(t)=\zeta_{s}^{q, b}(t)$ is the zeta function defined by (12.1) for the hermitian vector bundle $E_{s} \otimes \bigwedge^{a} \bar{T}_{s}^{*}$ (instead of $E$ there). Then we define the Quillen metric $k^{b}$ on $\lambda^{b}$ by

$$
k_{s}^{b}={ }^{\prime} k_{s}^{b} \tau_{s}, \quad s \in U^{b} .
$$

If we take another positive number $c$ and the corresponding open subset $U^{c}$ of $S$, then exactly as in [B-G-S III; $\S 1, \mathrm{~b})$, d)] we can construct a natural hermitian isomorphism $\varphi_{b c}:\left(\lambda_{b}, k_{b}\right) \stackrel{\sim}{\rightarrow}\left(\lambda_{c}, k_{c}\right)$ which is smooth on the smooth locus $U_{r e g}^{b c}$ of $U^{b c}:=U^{b} \cap U^{c}$. Via the isomorphisms $\varphi_{b c}$ for various $b$ and $c, \lambda^{b}$ on $U^{b}$ patch together to give a hermitian complex line bundle $(\lambda, k)$ on the whole $S$. On the smooth locus $S_{\text {reg }}$ it gives precisely the one constructed in [B-G-S III; $\S 1]$ as is clear from the construction.

Proposition 12.3. The hermitian line bundle $(\lambda, k)$ constructed as above has a natural $C^{\infty}$ structure which restricts on $S_{\text {reg }}$ to the canonical one constructed in [B-G-S III].

Proof. We consider the following situation, which is always realized locally in a neighborhood of any point of $S: 1) S$ is an analytic subset of a domain $D$ in some $\mathbb{C}^{N} .2$ ) There exist a $C^{\infty}$ trivialization $(\psi, \tilde{\psi})$ of $(f: \mathscr{X} \rightarrow S, E)$ over $S$ as follows;

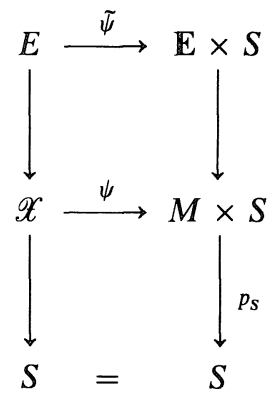

where $M$ is the underlying $C^{\infty}$ manifold of $X_{s}$ for $s \in S$ and $\mathbb{E} \rightarrow M$ is the underlying $C^{\infty}$ complex vector bundle of $E_{s} \rightarrow X_{s}$. 3) There exist a $C^{\infty}$ family $\left\{J_{y}\right\}_{y \in D}$ of almost complex structures $J_{y}$ on $M$ and a $C^{\infty}$ family $\left\{\widetilde{J}_{y}\right\}_{y \in D}$ of almost complex structures on $\mathbb{E}$ which are induced for $y \in S$ by the isomorphism $\left(\psi_{y}, \tilde{\psi}_{y}\right):\left(X_{y}, E_{y}\right) \stackrel{\sim}{\rightarrow}(M, \mathbb{E})$. 4) There exist a $C^{\infty}$ family $\left\{g_{y}\right\}_{y \in D}$ of $C^{\infty}$ almost hermitian metrics $g_{y}$ on the almost complex manifold $\left(M, J_{y}\right)$ and a $C^{\infty}$ family $\left\{h_{y}\right\}_{y \in D}$ of hermitian metrics on $E_{y}$ which are induced for $y \in S$ from the corresponding ones on $X_{s}$ and $E_{s}$ via $\left(\psi_{s}, \tilde{\psi}_{s}\right)$. Let $D_{y}^{q}(\mathbb{E})$ be the space of $\mathbb{E}$-valued $C^{\infty}(0, q)$-forms on the almost complex manifold $\left(M, J_{y}\right)$ and let $\bar{\partial}_{y}^{q}: D^{q}(\mathbb{E}) \rightarrow$ 
$D^{q+1}(\mathbb{E})$ be induced by $\left(J_{y}, \widetilde{J}_{y}\right)$. By $(\psi, \tilde{\psi})$, for $s \in \mathbb{S}$ this is identified with the Dolbeault operator on $X_{s}$.

In conclusion, the above consideration shows that the objects $D^{q}\left(E_{s}\right), \bar{\partial}_{s}^{q}: \mathbb{D}^{q}\left(E_{s}\right) \rightarrow$ $D^{q+1}\left(E_{s}\right), \bar{\partial}_{s}^{*}, \square_{s}^{q}$ defined naturally for $s \in S$ extends smoothly to a $C^{\infty}$ family of similar objects parametrized by $D$; in particular $\square_{y}^{q}$ is defined for all $y \in \mathbb{D}$ and may be assumed to be strongly elliptic by restricting $D$ if necessary. Now by using these extended objects we define $\widetilde{U}^{b} \subseteq D$ and $K_{y}^{q, b}$ for $y \in D$ in the same way as above. Then $\mathbb{K}_{D}^{q, b}:=$ $\bigcup_{y \in D} K_{y}^{q, b}$ has a natural structure of a $C^{\infty}$ complex vector bundle on the open subset

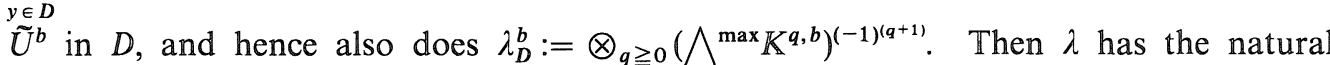
structure of a $C^{\infty}$ complex line bundle as the restriction of $\lambda_{D}^{b}$ to $S$.

Further, if we define the Quillen metric on $\lambda_{D}^{b}$ by the same formulae as in (12.2) and (12.3), then by Proposition 12.1 it is smooth over $D$, and hence its restriction to $S$, which is exactly the Quillen metric on $\lambda^{b}$ on $U^{b}$, is also smooth. Finally, the fact that $\varphi_{b c}$ is smooth can be shown similarly as in the smooth case.

Denote by $\mathscr{D}_{\mathscr{X} / S}^{q}(E)$ the sheaf of $E$-valued relative $C^{\infty}(0, q)$-forms on $\mathscr{X}$. We may consider the relative Dolbeault complex $\left(\mathscr{D}_{\mathscr{X} / S}^{\circ}(E), \bar{\partial}_{\mathscr{X} / S}\right)$, which is a fine resolution if the sheaf $\mathcal{O}_{\mathscr{X} / S}(E)$ by Proposition 1.4, where $\mathcal{O}_{\mathscr{X} / S}(E)$ is the sheaf of $C^{\infty}$ sections of $E$ which are holomorphic on each fiber of $f . \quad$ Let $\mathscr{K}^{q, b}$ be the sheaf of $\mathbb{C}^{\infty}$ sections of the $C^{\infty}$ vector bundle $K^{q, b}$ on $S$. Then we have the natural inclusion of complex of $\mathscr{D}_{S^{-}}$ modules

$$
l^{\circ}: \mathscr{K}^{\circ, b} \rightarrow f_{*} \mathscr{D}_{\mathscr{X} / S}^{\circ}(E) .
$$

Lemma $12.40 i^{\circ}$ is a quasi-isomorphism.

Proof. This is due to $[\mathbb{B}-G-\mathbb{S}$ III; Lemma 3.8] when $S$ is nonsingular. In the general case the proof is essentially the same. We use the notation of the proof of the previous proposition. First, note that $\mathscr{K}^{\circ, b}$ extends to a locally free $\mathscr{D}_{D}$-module $\mathscr{K}_{D}^{\circ, b}$ as the sheaf of $C^{\infty}$ sections of $\mathbb{K}_{D}^{\circ, b} \rightarrow D$. Similarly, the Dolbeault sheaf $f_{*} \mathscr{D}_{\mathscr{X} / S}^{\circ}(E)$ extends to the Dolbeault sheaf $\tilde{f}_{*} \mathscr{D}_{Z / D}^{\circ}(E)$ on the whole $D$, where $\mathbb{Z}=\mathbb{M} \times \mathbb{D}$ and $\tilde{f}_{D}: \mathbb{Z} \rightarrow \mathbb{D}$ is the natural projection. Finally, $l^{\circ}$ also extends to a natural inclusion $l_{D}^{\circ}: \mathscr{K}^{\circ, b} \rightarrow$ $f_{*} \mathscr{D}_{Z / D}^{\circ}(E)$ of sheaves of $\mathscr{D}_{D}$-modules.

Now for any $y \in Y$ let $P_{y}: D_{y}^{q}(E) \rightarrow K_{y}^{q, b}$ be the orthogonal projection and $G_{y}: D_{y}^{q}(E) \rightarrow$ $D_{y}^{q}(E)$ the Green operator for the strongly elliptic operator $\square_{y}^{q}$. These depend smoothly on $y$ (cf. [Ko-Mo; p. 177]) and give therefore the maps $\mathbb{P}: \tilde{f}_{*} \mathscr{D}_{Z / D}^{q}(E) \rightarrow \mathscr{K}_{D}^{q, b}$ and $G: \tilde{f}_{*} \mathscr{D}_{Z / D}^{q}(E) \rightarrow \tilde{f}_{*} \mathscr{D}_{Z / D}^{q}(E)$ of sheaves. Finally, as in $[\mathbb{B}-G-S] \bar{\partial}_{y}^{*} G_{y}\left(1-P_{y}\right)$ gives a $C^{\infty}$ homotopy between 1 and $P$, in view of the formula

$$
\bar{\partial}_{y} \bar{\partial}_{y}^{*} G_{y}\left(1-P_{y}\right)+\bar{\partial}_{y}^{*} G_{y}\left(1-P_{y}\right) \bar{\partial}_{y}=1-P_{y}, \quad y \in \mathbb{D}
$$

Now we want to apply the theory of a relative analytic space of Forster- $\mathbb{K}$ norr $[\mathrm{F}-\mathrm{K}]$ to the induced morphism of ringed spaces:

$$
f^{\infty}:\left(\mathscr{X}, \mathcal{O}_{\mathscr{X} / S}\right) \rightarrow\left(S, \mathscr{D}_{S}\right) .
$$

We have to check that $f^{\infty}$ satisfies the necessary conditions of $[\mathbb{F}-\mathbb{K}]$. We use freely the terminology of $[\mathrm{F}-\mathbb{K}]$. 
Let $U \subseteq S$ be an open subset embedded in a domain $D$ in $\mathbb{C}^{m}$ as a closed analytic subset. Denote by $\mathscr{J} \subseteq \mathscr{D}_{D}$ the sheaf of $C^{\infty}$ functions that vanish on $U$. Then $\Gamma(\mathscr{D}, \mathscr{J})$ is a closed ideal of the $m F$-algebra $\Gamma\left(D, \mathscr{D}_{D}\right)$. (Roughly, a Fréchet algebra whose topology is defined by multiplicative seminorms (cf. [F-K; p.118])). We shall endow $\Gamma\left(U, \mathscr{D}_{U}\right)=\Gamma\left(D, \mathscr{D}_{D}\right) / \Gamma(U, \mathscr{J})$ with the quotient $m F$-algebra structure, which is independent of the embedding as above. Then it is immediate to see:

Lemma 12.5. The ringed space $\left(S, \mathscr{D}_{S}\right)$ has the natural structure of an $m F B$-space ([F-K, p.119]) such that for any open subset $U \subseteq S$ as above the corresponding $m F$-algebra structure on $\Gamma\left(U, \mathscr{D}_{S}\right)$ is the one defined above. Moreover, it is of type $(L)$ in the sense that every point admits a neighborhood basis $\mathfrak{B}=\{V\}$ satisfying $H^{q}\left(V, \mathscr{D}_{S}\right)=0$ for all $q>0$.

Next we consider the $m F B$-structure on $\left(\mathscr{X}, \mathcal{O}_{\mathscr{X} / S}\right)$. Let $P$ be a polydisc in $\mathbb{C}^{n}$ and $U \subseteq S$ any open subset. Then we have a natural isomorphism

$$
\Gamma\left(U, \mathscr{D}_{S}\right) \hat{\otimes} \Gamma\left(P, \mathcal{O}_{P}\right) \underset{\rightarrow}{\rightarrow}\left(U \times P, \mathcal{O}_{(U \times P) / U}\right),
$$

which defines an $m F B$ algebra structure on the latter (cf. [F-K; p.121]), where $\hat{\otimes}$ denotes the topological tensor product. As [F-K; Prop. 1.1] shows, for any open subset $D \subseteq \mathbb{C}^{n}$ this introduces a natural $m F B$-structure on the ringed space $(U \times$ $\left.D, \mathcal{O}_{(U \times D) / U}\right)$. The proof of the following lemma is also straightforward:

Lemma 12.6. The ringed space $\left(\mathscr{X}, \mathcal{O}_{\mathscr{X} / S}\right)$ admits a natural structure of an $m F B$ space such that on any open subset $V$ of $\mathscr{X}$ which is isomorphic over an open subset $U$ of $S$ to the product $U \times D$ the induced $m F B$-structure coincides with the one induced from $\left(U \times D, \mathcal{O}_{(U \times D) / U}\right)$ via the given isomorphism. Moreover the morphism of $m F B$-spaces $f^{\infty}:\left(\mathscr{X}, \mathcal{O}_{\mathscr{X} / S}\right) \rightarrow\left(S, \mathscr{D}_{S}\right)$ is smooth, so that in particular $\left(\mathscr{X}, \mathcal{O}_{\mathscr{X} / S}\right)$ is a relative analytic space $\operatorname{over}\left(S, \mathscr{D}_{S}\right)(c f .[\mathrm{F}-\mathbb{K} ; \S 3])$.

Let now $\mathscr{E}$ be the $\mathcal{O}_{\mathscr{X}}$-module of germs of holomorphic sections of $E$. Set $\mathscr{E} \infty=$ $\mathscr{E} \otimes_{\mathcal{O}_{\mathscr{T}}} \mathcal{O}_{\mathscr{X} / \mathrm{S}}=\mathcal{O}_{\mathscr{X} / \mathrm{S}}(E)$ for brevity. In view of Lemmas 12.5 and 12.6 we can apply Theorem II of $[\mathrm{F}-\mathrm{K}]$ to the pairs $(f, \mathscr{E})$ and $\left(f^{\infty}, \mathscr{E}^{\infty}\right)$.

Lemma 12.7. $R f_{*} \mathscr{E}$ (resp. $\left.R f_{*}^{\infty} \mathscr{E}^{\infty}\right)$ is a perfect complex of $\mathcal{O}_{\mathbf{S}^{-}}\left(\right.$resp. $\mathscr{D}_{\mathbf{S}^{-}}$) modules in the sense that locally on $S$ it is quasi-isomorphic to a bounded complex of finite free $\mathcal{O}_{\mathbf{S}^{-}}\left(\right.$resp. $\left.\mathscr{D}_{\mathbf{S}^{-}}\right)$modules.

By virtue of this lemma we can apply the theory of determinants of KnudsenMumford [K-M] to $R f_{*}^{\infty} \mathscr{E}$ as well as to $R f_{*} \mathscr{E}$ so that, besides det $R f_{*} \mathscr{E}$, we can speak also of the determinant det $R f_{*}^{\infty} \mathscr{E}$, which is an invertible $\mathscr{D}_{S}$-module. (See [B-G-S III; $\S 3, a)]$ for a discussion about extending the theory of $[\mathrm{K}-\mathrm{M}]$ from schemes to complex manifolds. More generally one easily checks that Theorems 1 and 2 of [K-M] hold true without any change on any analytic space $S$, or on the associated $C^{\infty}$ space $\left(S, \mathscr{D}_{S}\right)$.) Now we set

$$
\underline{\lambda}^{\mathrm{KM}}=\left(\operatorname{det} R f_{*} \mathscr{E}\right)^{-1}, \quad \text { and } \quad \underline{\lambda}_{\infty}^{\mathrm{KM}}=\left(\operatorname{det} R f_{*}^{\infty} \mathscr{E} \infty\right)^{-1} .
$$

Then our next purpose is to show the following: 
Proposition 12.8 . There exists a canonical isomorphism of $\mathscr{D}_{\mathbf{S}}$-modules $\underline{\lambda}^{\mathrm{KM}} \otimes_{\mathcal{O}_{S}} \mathscr{D}_{\mathbf{S}} \rightarrow$ $\lambda_{\infty}^{\mathrm{KM}}$.

For any $\mathcal{O}_{X}$-module $\mathscr{F}$ we get a natural homomorphism of $\mathscr{D}_{S}$-modules $f_{*} \mathscr{F} \otimes_{\mathcal{O}_{S}}$ $\mathscr{D}_{S} \rightarrow f_{*}^{\infty}\left(\mathscr{F} \otimes_{\mathcal{O}} \mathcal{O}_{\mathscr{X} / S}\right)$, giving the morphism of functors $\left(-\otimes_{\mathcal{O}_{S}} \mathscr{D}_{S}\right) \cdot f_{*} \rightarrow f_{*}^{\infty} \cdot\left(-\otimes_{\mathcal{O}}\right.$ $\left.\mathcal{O}_{\mathscr{X} / \mathrm{S}}\right)$. This induces in the derived category a homomorphism

$$
a:\left(R f_{*} \mathscr{E}\right) \otimes_{\mathcal{O}_{S}}^{L} \mathscr{D}_{S} \rightarrow R f_{*}^{\infty} \mathscr{E}^{\infty}
$$

Then we shall first prove:

Proposition 12.9. The canonical homomorphism a above is quasi-isomorphic.

Proof. Since the problem is local, we may assume by Lemma 12.7 that there exist 1) a bounded free complex $\mathscr{P}^{\circ}\left(\right.$ resp. $\left.\mathscr{Q}^{\circ}\right)$ of finite free $\mathcal{O}_{S^{-}}$(resp. $\mathscr{D}_{S^{-}}$) module which realize $R f_{*} \mathscr{E}$ (resp. $\left.R f_{*}^{\infty} \mathscr{E}\right)^{\infty}$ ) in the derived category, and 2) an $\mathcal{O}_{S}$-linear map $u^{\circ}: \mathscr{P}^{\circ} \rightarrow \mathscr{Q}^{\circ}$ of complexes which realizes the canonical homomorphism $R f_{*} \mathscr{E} \rightarrow R f_{*}^{\infty} \mathscr{E}$ induced by the inclusion $\mathscr{E} \subset \mathscr{E}^{\infty}$. Then $u^{\circ}$ induces a $\mathscr{D}_{S}$-linear map $u_{\infty}^{\circ}: \mathscr{P}_{\infty}^{\circ}:=\mathscr{P}^{\circ} \otimes_{\mathcal{O}_{S}} \mathscr{D}_{S} \rightarrow \mathscr{D}^{\circ}$ which realizes the map $a$. It thus suffices to show that $u_{\infty}^{\circ}$ is quasi-isomorphic.

Since $\mathscr{P}^{\circ}$ and $\mathscr{Q}^{\bullet}$ consist of free modules, this follows if we show that for any $s \in S$ the induced map $u^{\circ}(s): \mathscr{P}^{\circ}(s) \rightarrow \mathscr{Q}^{\circ}(s)$ is quasi-isomorphic, where $\mathscr{P}^{\circ}(s)=\mathscr{P}^{\circ} \otimes_{\mathcal{O}_{s}} \mathbb{C}(s)$ and $\mathscr{2}^{\circ}(s)=\mathscr{2}^{\circ} \otimes_{\mathscr{D}_{S}} \mathbb{C}(s)$ and also note that $u^{\circ}(s):=u_{\infty}^{\circ} \otimes_{\mathscr{D}_{s}} \mathbb{C}(s)=u^{\circ} \otimes_{\mathcal{O}_{s}} \mathbb{C}(s)$. (Use the mapping cone of $u_{\infty}^{\circ}$ and reduce the problem to showing that a finite bounded free complex is exact if it is exact after tensorizing with $\mathbb{C}(s)$ for any $s$.)

For the latter purpose we shall realize $a$ by using Čech complex. Take and fix a locally finite Stein open covering $\mathfrak{B}=\left\{V_{l}\right\}$ of $\mathscr{X}$. For any open subset $U$ of $S$ we consider the Čech complexes $C^{\circ}\left(\mathfrak{B} \mid f^{-1}(U), \mathscr{E}\right)$ and $C^{\circ}\left(\mathfrak{B} \mid f^{-1}(U), \mathscr{E}^{\infty}\right)$ on $f^{-1}(U)$. Then after passing to sheaves these give rise to an $\mathcal{O}_{S}$-module $\mathscr{C}^{\circ}(\mathfrak{B}, \mathscr{E})$ and a $\mathscr{D}_{S}$-module $\mathscr{C}^{\circ}\left(\mathfrak{B}, \mathscr{E}^{\infty}\right)$ respectively on $S$ which realize $R f_{*} \mathscr{E}$ and $R f_{*}^{\infty} \mathscr{E}$ respectively. Moreover, the natural inclusion $j: \mathscr{C}^{\circ}(\mathfrak{B}, \mathscr{E}) \rightarrow \mathscr{C}^{\circ}\left(\mathfrak{B}, \mathscr{E}^{\infty}\right)$ realizes the natural map $R f_{*} \mathscr{E} \rightarrow \mathbb{R} f_{*}^{\infty} \mathscr{E}^{\infty}$. Since $\mathscr{P}^{\circ}$ and $\mathscr{2}^{\circ}$ are bounded complexes of finite free modules, we can get a commutative diagram

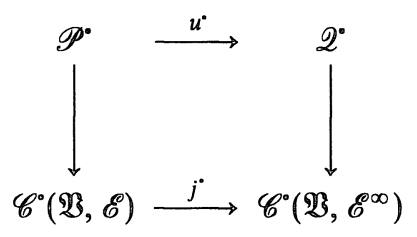

where the vertical homomorphisms are quasi-isomorphic. Then by tensorizing $\mathbb{C}(s)$ over $\mathcal{O}_{S}$ and over $\mathscr{D}_{S}$ respectively, we obtain a commutative diagram

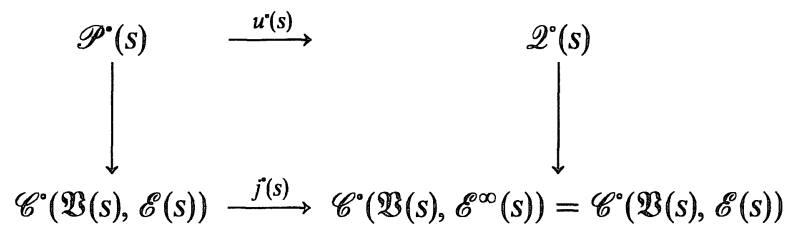

where $\mathfrak{B}(s)=\left\{V_{l} \cap X_{s}\right\}$. Here, the vertical arrows are again quasi-isomorphic since the relevant modules are either $\mathcal{O}_{S^{-}}$or $\mathscr{D}_{S^{-}}$flat modules; furthermore $j^{\circ}(s)$ is the identity. Thus $u^{\circ}(s)$ is quasi-isomorphic. 
Proof of Proposition 12.8. By taking the determinant of both sides of (12.4), we get natural isomorphisms

$$
\left(\operatorname{det} R f_{*} \mathscr{E}\right) \otimes_{\mathcal{O}_{S}} \mathscr{D}_{S} \cong \operatorname{det}\left(\left(R f_{*} \mathscr{E}\right) \otimes_{\mathcal{O}_{S}} \mathscr{D}_{S}\right) \stackrel{\sim}{\rightarrow} \operatorname{det} R f_{*}^{\infty} \mathscr{E}^{\infty},
$$

which is in fact realized by the isomorphisms

$$
\left(\operatorname{det} \mathscr{P}^{\circ}\right) \bigotimes_{\mathcal{O}_{S}} \mathscr{D}_{S} \cong \operatorname{det}\left(\mathscr{P}^{\circ} \otimes_{\mathcal{O}_{S}} \mathscr{D}_{S}\right) \stackrel{\operatorname{det} u_{\infty}^{\circ}}{\longrightarrow} \operatorname{det} \mathscr{Q}^{\cdot}
$$

in the notations of the above proof, where det denotes the Knudsen-Mumford determinant of complexes. The proposition clearly follows from (12.5).

Lemma 12.10. There exists a canonical isomorphism $w: \underline{\lambda} \rightarrow \underline{\lambda}^{\mathrm{KM}}$ of $\mathscr{D}_{\mathrm{s}}$-modules, where $\underline{\lambda}$ is the invertible $\mathscr{D}_{\mathrm{S}}$-module corresponding to $\lambda$.

Proof. By Lemma 12.4 the natural inclusion $\mathscr{K}^{\bullet, b} \hookrightarrow f_{*}^{\infty} \mathscr{D}_{\mathscr{X} / S}^{\circ}(E)$ is quasi-isomorphic on $U^{b}$. On the other hand, since $\mathscr{D}_{\mathscr{X} / S}^{\circ}(E)$ is a fine resolution of $\mathscr{E}^{\infty}$ we get canonical isomorphisms

$$
R f_{*}^{\infty} \mathscr{E} \stackrel{\sim}{\rightarrow} R f_{*}^{\infty} \mathscr{D}_{\mathscr{X} / S}^{\circ}(E) \stackrel{\sim}{\rightarrow} f_{*}^{\infty} \mathscr{D}_{\mathscr{X} / S}^{\circ}(E)
$$

Thus $\mathscr{K}^{\bullet, b}$ and $R f_{*}^{\infty} \mathscr{E}^{\infty}$ are canonically quasi-isomorphic. By taking the determinant of both complexes we get a canonical isomorphism $w^{b}: \underline{\lambda}^{b} \rightarrow \underline{\lambda}_{\infty}^{\mathrm{KM}}$. Moreover, on $U^{b c}$ we have $w^{b}=w^{c} \varphi_{b c}$. Hence, $w^{b}$ patch together to give a desired isomorphism $w$ as above.

Together with Proposition 12.8 this yields:

Proposition 12.11. There exists a natural isomorphism $\underline{\lambda} \widetilde{\sim}^{\mathrm{KM}} \underline{\mathrm{O}} \mathscr{\mathscr { D }}_{\mathrm{S}}$ which restricts on the smooth locus $S_{\text {reg }}$ to the one defined in [B-G-S III; Cor. 3.9].

The last assertion follows from the constructions.

Proof of Theorem 10.1. By definition $\lambda(E)$ is the holomorphic line bundle corresponding to the invertible $\mathscr{D}_{S}$-module $\underline{\lambda}^{\mathrm{KM}}$. By the isomorphism $\lambda \widetilde{\rightarrow} \lambda(E)$ as $C^{\infty}$ line bundles given by the above proposition, the Quillen metric $k$ is mapped to the Quillen metric $k_{E}$ on $\lambda(E)$ by the definitions. Hence, $k_{E}$ is of class $C^{\infty}$ by Proposition 12.3. Moreover, by Theorem BGS the chern form $c_{1}\left(\lambda(E), k_{E}\right)$ coincides with the degree 2 part of the fiber integral

$$
\int_{\mathscr{X} / S} \operatorname{ch}(E, h) \operatorname{td}(\mathscr{X} / S, g)
$$

on $S_{\text {reg }}$. Hence if the latter is locally $\partial \bar{\partial}$-exact on the whole $S$, it coincides with $c_{1}\left(\lambda(E), k_{E}\right)$ on the whole $S$ by Corollary 1.2 .

\section{Appendix}

We shall give a proof of the relative Dolbeault and Poincare lemmas as stated in Proposition 1.4. We shall follow the argument of $[A-G]$ in the case where the base space is smooth, but refer to general results on topological vector spaces as is exposed, e.g., in $[\mathrm{Bu}]$.

In general let $D$ be a polydisc in some $\mathbb{C}^{m}$ and $S$ an analytic subset of $D$. Let $\mathscr{J}$ be 
the ideal sheaf of $S$ in $\mathscr{D}_{D}$. Then the exact sequence

$$
0 \rightarrow \Gamma(\mathbb{D}, \mathscr{J}) \rightarrow \Gamma\left(D, \mathscr{D}_{D}\right) \rightarrow \Gamma\left(U, \mathscr{D}_{S}\right) \rightarrow 0
$$

turns $\mathscr{D}_{S}$ into a Fréchet sheaf since $\Gamma\left(\mathbb{D}, \mathscr{D}_{D}\right)$ is endowed with the usual Fréchet topology such that $\Gamma(D, \mathscr{J})$ is a closed subspace. Note also that $\Gamma\left(S, \mathscr{D}_{S}\right)$ is nuclear as well as $\Gamma\left(D, \mathscr{D}_{D}\right)(\mathrm{cf}$. $[\mathrm{Bu}$; Lemma 5.1$])$.

For a complex $\mathbb{F}$ réchet space $E$ we shall denote by $\Gamma(S, E)$ the space of $C^{\infty}$ maps of $S$ into $E$, i.e., the maps which extend locally at each point to a $\mathbb{C}^{\infty}$ map from its neighborhood in $D$.

Lemma $\mathbb{A}_{0} \mathbb{1}$. There exists a canonical isomorphism $\Gamma\left(S, \mathscr{D}_{S}\right) \bigotimes_{\varepsilon} \mathbb{E} \rightarrow \Gamma(S, E)$, where $\bigotimes_{\varepsilon}$ is the $\varepsilon$-tensor product in the sense of Grothendieck (cf. $\left.[\mathrm{Bu}]\right)$.

Proof. We consider the following commutative diagram of Fréchet spaces with exact rows:

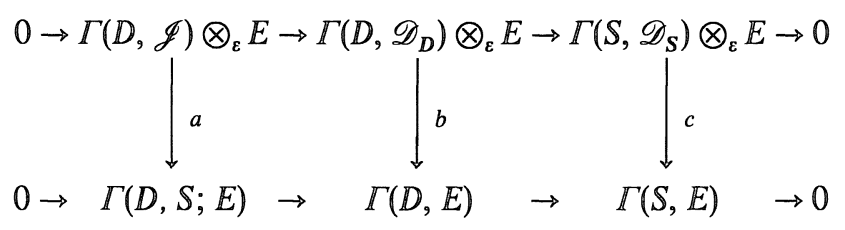

where $\Gamma(D, S ; E)=\{\psi \in \Gamma(D, E) ; \psi(s)=0$ for any $s \in S\}$, and the vertical maps are the natural ones (cf. [ $\mathrm{Bu}$; Prop. 3.1]). (Here the first sequence is exact since $\Gamma\left(S, \mathscr{D}_{S}\right)$ is nuclear (cf. [ $\mathrm{Bu}$; Th. 5.3])). Since $b$ is known to be isomorphic, it suffices to show that $a$ is isomorphic, i.e., surjective. But by the definition of $\varepsilon$-product, for any $f \in \Gamma(D, E) f$ is in the image of $a$ if and only if of vanishes identically on $S$ for any continuous linear form $v$ on $E$ (cf. the proof of [ $\mathrm{Bu}$; Prop. 10.3]). It follows that $a$ is surjective.

Since $\Gamma\left(S, \mathscr{D}_{S}\right) \otimes_{\varepsilon}$-is an exact functor $([\mathrm{Bu} ;$ Th. 5.3]) we get:

Corollary $\mathbb{A}_{0}$ 2. $E \rightarrow \Gamma(S, E)$ is an exact functor from the category of Fréchet spaces to itself.

Proof of Proposition 1.4. We treat only the case of Dolbeault complex. (The other two cases are proved in a similar way.) The problem is local: so we may assume that $f$ is a projection $f: X=U \times S \rightarrow S$, where $U$ is a polydisc in $\mathbb{C}^{n}$ and $\mathbb{S}$ is a closed analytic subset of a polydisc $\mathbb{D}$ of $\mathbb{C}^{m}$. We first see that there exist natural isomorphisms $\Gamma\left(X, \mathscr{D}_{X}\right) \cong \Gamma\left(S, \Gamma\left(U, \mathscr{D}_{U}\right)\right)$ and $\Gamma\left(X, \mathcal{O}_{X / S}\right)=\Gamma\left(S, \Gamma\left(U, \mathcal{O}_{U}\right)\right)$. We show this for the first case since the proof is parallel in the other case. We consider the following commutative diagram of exact sequences (cf. Cor. A.2)

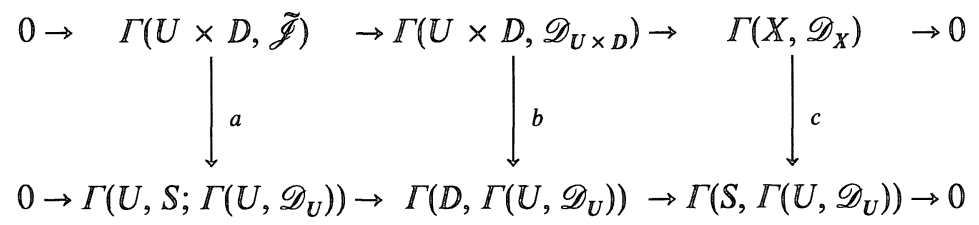


where $\tilde{J}$ is the ideal sheaf of $X$ in $\mathscr{D}_{U \times D}$. Here, it is immediate to see that $a$ and $b$ are isomorphic, and hence $c$ also is isomorphic.

Now in order to prove the proposition it suffices to show that the sequence

$$
0 \rightarrow \Gamma\left(X, \mathcal{O}_{X / S}\right) \rightarrow \Gamma\left(X, \mathscr{D}_{X}\right) \rightarrow \Gamma\left(X, \mathscr{D}_{X / S}^{1}\right) \rightarrow
$$

is exact since $X$ is arbitrary. By virtue of what we have proved above this sequence is isomorphic to

$$
0 \rightarrow \Gamma\left(S, \Gamma\left(U, \mathcal{O}_{U}\right)\right) \rightarrow \Gamma\left(S, \Gamma\left(U, \mathscr{D}_{U}\right)\right) \rightarrow \Gamma\left(S, \Gamma\left(U, \mathscr{D}_{U}^{1}\right)\right) \rightarrow
$$

which is exact by the above corollary since

$$
0 \rightarrow \Gamma\left(U, \mathcal{O}_{U}\right) \rightarrow \Gamma\left(U, \mathscr{D}_{U}\right) \rightarrow \Gamma\left(U, \mathscr{D}_{U}^{1}\right) \rightarrow
$$

is exact, where $\mathscr{D}_{U}^{q}$ is the Dolbeault sheaf of $(0, q)$-forms on $U$.

\section{References}

[ A ] Ahlfors, L. V., Some remarks on Teichmüller space of Riemann surfaces, Ann. of Math., 74 (1961), 171-191.

[A-D-N] Agmon, S., Douglis, A. and Nirenberg, L., Estimates near boundary for the solutions of elliptic partial differential equations satisfying general boundary conditions II, Comm. Pure Appl. Math., 17 (1964), 35-92.

[ A-G ] Andreotti, A. and Grauert, H., Théorème de finitude pour la cohomologie des espaces complexes, Bull. Soc. Math. France, 90 (1962), 193-259.

[ At ] Atiyah, M. F., Complex analytic connections in fiber bundles, Trans. Amer. Math. Soc., 85 (1957), 181-207.

[ $\mathrm{Ba}$ ] Baily, W. L., The decomposition theorem for $V$-manifolds, Amer. J. Math., 78 (1956), 862-888.

[ Bea ] Beauville, A., Variétés kählériennes dont la première classes de Chern est nulle, J. Diff. Geom., 18 (1983), 755-782.

[ B-E ] Berger, M. and Ebin, D. G., Some decompositions on the spaces of symmetric tensors on a Riemannian manifold, J. Diff. Geom., 3 (1969), 379-392.

[ Bes ] Besse, A. L., Einstein manifolds, A series of Modern Surveys on Math. 10, Springer, Berlin Heidelberg New York: 1986.

[ $\mathrm{Bi}$ ] Bismut, J. M., The Atiyah-Singer index theorem for families of Dirac operators: Two heat equation proofs, Invent. math., 83 (1986), 91-151.

[ B-F ] Bismut, J. M. and Freed, D. S., The analysis of elliptic families I, Comm. Math. Phys., 106 (1986), 159-176.

[ B-G-S ] Bismut, J. M., Gillet, H. and Soulé, Ch., Analytic torsion and holomorphic determinant bundles I, II, III, Commun. Math. Phys., 115 (1987), 49-78, 79-126, 301-351.

[ $\mathrm{Bu}$ ] Bungart, L., Holomorphic functions with values in locally convex spaces and applications to integral formulas, Trans. Amer. Math. Soc., 111 (1964), 317-344.

[ B-B ] Burns, D. and de Bartolomeis, P., Stability of vector bundles and extremal metrics, Invent. math., 92 (1988), 403-408.

[ C1 ] Calabi, E., Extremal Kähler metrics, In: Yau, S. T. (ed.) Seminars on differential geometry. Proceedings, Princeton 1979 (Ann. of Math. Studies, 102, pp. 259-290) Princeton Univ. Press \& Univ. of Tokyo 1982.

[ $\mathrm{C} 2$ ] - Extremal Kähler metrics II, In: Cheval, I., \& Farkas, H. M. (eds.) Differential geometry and complex analysis (dedicated to E. Rauch, pp. 95-114) Springer 1985.

[ D ] Donaldson, S. K., Infinite determinants, stable bundles and curvature, Duke Math. J., 54 (1987), 231-247. 
[ F ] Fischer, G., Complex analytic geometry, Lecture Notes in Math., 538 Springer Berlin Heidelberg New York: 1976.

[ F-K ] Forster, O. and Knorr, K., Relative-analytische Räume und die Kohärenz von Bildgarben, Invent. math., 16 (1972), 133-160.

[ Fu1 ] Fujiki, A., On automorphism groups of compact Kähler manifolds, Invent. math., 44 (1978), $226-258$.

[ Fu2 ] —, Kählerian normal complex surfaces, Tohoku Math. J., 35 (1983), 107-117.

[ Fu3 ] - Coarse moduli spaces for polarized compact Kähler manifolds, Publ. RIMS, Kyoto Univ., 20 (1984), 977-1005.

[ Fu4 ] — - Coarse moduli space for polarized algebraic manifolds, unpublished manuscript.

[ Fu5 ] — Extremal Kähler metrics and moment maps, in preparation.

[ Fu6 ] — Remarks on extremal Kähler metrics on ruled manifolds, preprint.

[Fu-Sch] Fujiki, A. and Schumacher, G., The moduli space of Kähler structures on a real compact symplectic manifold, Publ. RIMS, Kyoto Univ., 24 (1988), 141-168.

[ Gr ] Grauert, H., Über Modifikationen und exzeptionelle analytische Mengen, Math. Ann., 146 (1962), 331-368.

[ Gri ] Griffiths, P. A., The extension problem for compact submanifolds I, In: Aeppli et al. (eds.) Proc. of the Conf. on Complex Analysis, Minneapolis 1964 pp. 113-142, Springer 1965.

[ $\mathrm{H}$ ] Holmann, H., Komplexe Räume mit komplexen Transformations-gruppen, Math. Ann., 150 (1963), $327-360$.

[ K-M ] Knudsen, F. and Mumford, D., The projectivity of the moduli space of stable curves I: Preliminaries on "det" and "div", Math. Scand., 39 (1976), 19-55.

[ K ] Kobayashi, S., Transformation groups in differential geometry, Springer Berlin Heidelberg New York: 1972.

[ K-N ] Kobayashi, S. and Nomizu, K., Foundations of differential geometry II, Interscience Publ. New York London Sydney: 1969.

[Ko-Mo] Kodaira, K. and Morrow, J., Complex manifolds, Holt, Reinhart and Winston Inc., New York 1971.

[ Koi ] Koiso, N., Einstein metrics and complex structure, Invent. math., 73 (1983), 71-106.

[ $\mathrm{Ku}$ ] Kuranishi, M., Deformations of compact complex manifolds, Les Presses de L'univ. de Montréal 1971.

[ M ] Malgrange, B., Sur les fontions différentiables et les ensembles analytiques, Bull. Soc. Math. France, 91 (1963), 113-127.

[ Ma ] Matsusaka, T., Polarized varieties with a given Hilbert polynomial, Amer. J. Math., 94 (1972), 1027-1077.

[ $\quad \mathrm{P}$ ] Popp, H., Moduli theory and classification theory of algebraic varieties, Lecture Notes in Math., 620, Springer 1977.

[ S ] Schneider, M., Halbstetigkeitssätze für relativ analytische Räume, Invent. math., $\mathbb{1 6}$ (1972), 161 176.

[ Sch1 ] Schumacher, G., Eine Anwendung des Satzes von Calabi-Yau auf Famillien kompakter komplexer Mannigfaltigkeiten, Invent. math., 71 (1983), 295-307.

[ Sch2 ] - Construction of the coarse moduli space of compact polarized Kähler manifolds with $c_{1}=0$, Math. Ann., 264 (1983), 81-90.

[ Sch3 ] — - Moduli of polarized Kähler manifolds, Math. Ann., 269 (1984), 137-144.

[ Sch4 ] — - On the geometry of moduli spaces, Manuscripta math., 50 (1985), 229-269.

[ $\mathrm{Si}$ ] Siu, Y. T., Curvature of the Weil-Petersson metric in the moduli space of compact KählerEinstein manifolds of negative first chern class, Aspects of Math. 9: Friedr. Vieweg \& Sohn, Braunschweig/Wiesbaden 1986 pp. 261-298.

[ $\mathrm{Sp}$ ] Spallek, K., Differenzierbare und holomorphe Funktionen auf analytischen Mengen, Math. Ann., 161 (1965), 143-162.

[ V ] Varouchas, J., Kähler spaces and proper open morphisms, Math. Ann., 283 (1989), 13-52.

[ $\mathrm{Vi}$ ] Viehweg, E., Weak positivity and stability of certain Hilbert points, preprint MPI Bonn, 1988.

[ Wa ] Watson, B., Almost Hermitian submersions, J. Diff. Geom., 11 (1976), 147-165. 
[ We ] Wells, R. O., Differential analysis on complex manifolds, Graduate Texts in Math., Springer Berlin Heidelberg New York: 1979.

[ Wo ] Wolpert, S. A., Chern forms and the Riemann tensor for the moduli space of curves, Invent. math., 85 (1986), 119-144. 
